\title{
The Callerya Group redefined and Tribe Wisterieae (Fabaceae) emended based on morphology and data from nuclear and chloroplast DNA sequences
}

\author{
James A. Compton', Brian D. Schrire², Kálmán Könyves³, Félix Forest² \\ Panagiota Malakasi², Sawai Mattapha ${ }^{4}$, Yotsawate Sirichamorn ${ }^{5}$
}

I Spilsbury Farm, Tisbury, SP3 6RU, UK 2 Comparative Plant and Fungal Biology Department, Royal Botanic Gardens, Kew, Richmond, Surrey, TW9 3AE, UK 3 Herbarium, Royal Horticultural Society Garden, Wisley, Woking, Surrey, GU23 6QB, UK 4 Udon Thani Rajabhat University, Department of Biology, Faculty of Science, Udon Thani 41000, Thailand 5 Silpakorn University, Department of Biology, Faculty of Science, Sanam Chandra Palace campus, Nakhon Pathom 73000, Thailand

Corresponding author: James Compton (jamiecompton@madasafish.com)

Academic editor: P. Herendeen | Received 26 March 2019 | Accepted 21 May 2019 | Published 26 June 2019

Citation: Compton JA, Schrire BD, Könyves K, Forest F, Malakasi P, Mattapha S, Sirichamorn Y (2019) The Callerya Group redefined and Tribe Wisterieae (Fabaceae) emended based on morphology and data from nuclear and chloroplast DNA sequences. PhytoKeys 125: 1-112. https://doi.org/10.3897/phytokeys.125.34877

\begin{abstract}
The Tribe Wisterieae (Zhu 1994), founded on the single genus Wisteria, is emended and recircumscribed based on morphology and data from nuclear ITS and $n d h J-t r n F$, matK and $r b c L$ chloroplast DNA sequences. This newly enlarged tribe comprises 36 species and 9 infraspecific taxa within 13 described genera. Six genera are new, two are reinstated and five were previously placed in Tribe Millettieae. The genus Adinobotrys is also reinstated comprising two species including the new combination $A$. vastus. Other reinstated genera include Whitfordiodendron, with four species, and Padbruggea, with three species, including the reinstatement of $P$. filipes and the new combination $P$. filipes var. tomentosa. The existing genera Afgekia, Callerya, Endosamara (with the new combination E. racemosa var. pallida), Sarcodum and Wisteria, with the new combinations $W$. frutescens subsp. macrostachya are evaluated. The new genera comprise three Australasian species in Austrocallerya: A. australis, A. megasperma and A.pilipes; Wisteriopsis with five species from east Asia has six new combinations: W. japonica, W. kiangsiensis, W. championii, W. eurybotrya, W. reticulata and W. reticulata var. stenophylla. Two species comprise the new Thai genus Kanburia: $K$. tenasserimensis and $K$. chlorantha. Nanhaia comprises the two species: $N$. fordii and $N$. speciosa and the monotypic genera Sigmoidala and Serawaia are based respectively on the species S. kityana and $S$. strobilifera. Lectotypes are designated for the names Adinobotrys filipes, A. myrianthus, Millettia bonatiana, M. bracteosa, M. championii, M. cinerea, M. dielsiana, M. kityana, M. maingayi, M. nitida, M. oocarpa,
\end{abstract}

Copyright James A. Compton et al. This is an open access article distributed under the terms of the Creative Commons Attribution License (CC BY 4.0), which permits unrestricted use, distribution, and reproduction in any medium, provided the original author and source are credited. 
M. purpurea, M. reticulata, M. reticulata var. stenophylla, Padbruggea dasyphylla, Pterocarpus australis, Robinia racemosa, Whitfordiodendron scandens, W. sumatranum and Wisteria pallida. A neotype is designated for the name Millettia leiogyna.

\section{Keywords}

Tribe Wisterieae emended, Leguminosae, new genera, Austrocallerya, Kanburia, Nanhaia, Serawaia, Sigmoidala, Wisteriopsis, molecular phylogeny, morphological key

\section{Table of Contents}

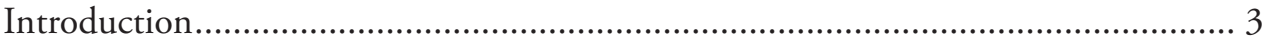

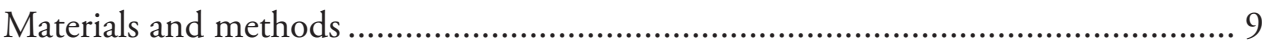

Molecular preparation and sequencing .......................................................... 9

Phylogenetic procedures and analyses........................................................... 16

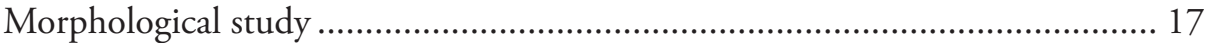

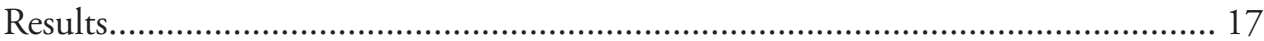

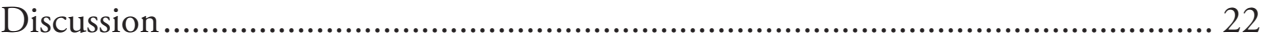

Morphology of the Callerya Group ............................................................... 22

Callerya group taxonomy 1: The genus Callerya including genera previously placed in synonymy prior to this study....................................................................... 25

Callerya group taxonomy 2: Additional genera within the Callerya group as defined in treatments prior to this study................................................................ 35

Callerya group taxonomy 3: New genera within the Callerya group as delimited in

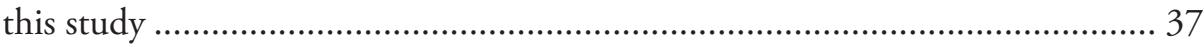

Integrating our results: the Callerya group to Tribe Wisterieae........................... 40

Taxonomic treatment of Tribe Wisterieae ......................................................... 43

Emended diagnosis of Tribe Wisterieae ................................................................ 44

Morphological key to the genera in Tribe Wisterieae together with Adinobotrys.... 44

Key to species of Adinobotrys.................................................................... 50

Clade A - Sarcodum, Endosamara and Sigmoidala........................................... 51

Key to species of Sarcodum ........................................................................ 52

Key to varieties of Endosamara racemosa ...................................................... 54

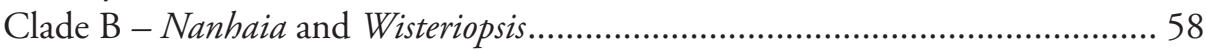

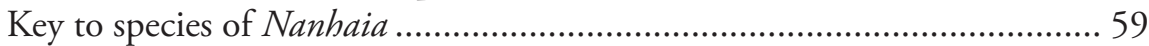

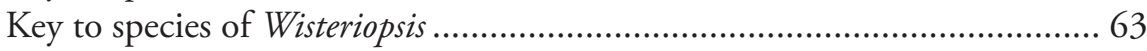

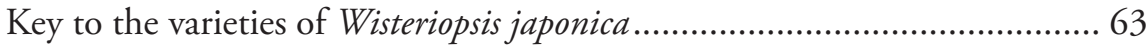

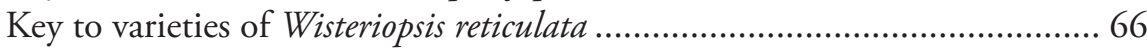

Clade C - Callerya, Serawaia, Whitfordiodendron, Kanburia and Afgekia .......... 68

Key to species of Callerya recognized in this treatment................................ 69

Key to varieties of Callerya nitida ............................................................... 70

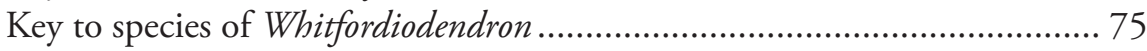

Key to species of Kanburia ..................................................................... 78 
Key to species of Afgekia ....................................................................... 80

Clade D - Padbruggea and Austrocallerya ............................................... 81

Key to species of Padbruggea ........................................................ 82

Key to varieties of Padbruggea filipes ..................................................... 84

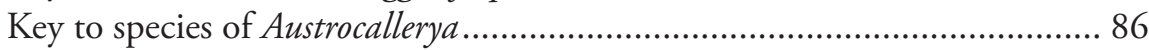

Clade E - Wisteria .......................................................................... 89

Key to species of Wisteria ................................................................ 91

Key to subspecies of Wisteria frutescens............................................... 91

Key to varieties of Wisteria sinensis .................................................... 95

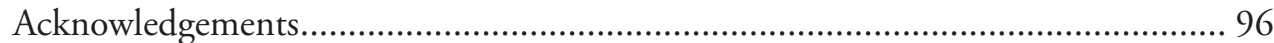

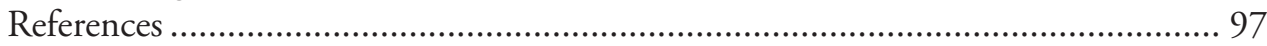

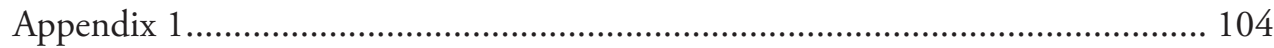

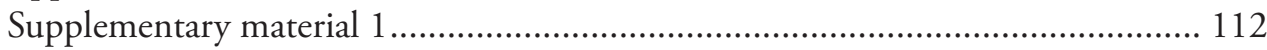

\section{Introduction}

The Tribe Millettieae was first described by Miquel (1855: 137), based on the type genus Millettia Wight \& Arn. (Wight and Arnott 1834: 263). This genus of six species was characterised largely by the pods of the southern Indian type species $M$. rubiginosa Wight \& Arn. Miquel emphasised the compressed nature of the pods as a significant distinguishing character and his tribal description very loosely defined the new Tribe Millettieae, which included eight genera: Brachypterum (Wight \& Arn.) Benth., Derris Lour., Pongamia Adans., Padbruggea Miq., Aganope Miq., Millettia, Otosema Benth. and Mundulea Benth. (Miquel 1855: 137).

Miquel (1855: 137) stated:

stamens monadelphous or diadelphous; calyx campanulate, more or less bilabiate; seed pod indehiscent, woody or leathery, sometimes opening tardily; leaves opposite and pinnate; flowers in racemes

Millettia was, furthermore, distinguished from the genera Pongamia Adans. and Dalbergia L.f. by the legume being compressed around the seeds and by the fruit's thick woody texture (Wight and Arnott 1834: 263). Dunn (1912a) in his revision of Millettia placed the genus in Tribe Galegeae (Bronn) Torr. \& Gray subtribe Tephrosiinae Benth. (as Subtribe "Tephrosieae”). Geesink (1984: 3) described Tribe Millettieae with the characters:

Inflorescence of panicles, pseudopanicles or derived pseudopanicles; wing petals adherent to the keel; keel petals usually valvately connate; pod dehiscent or indehiscent; seed chamber mostly absent; seeds 1 or few; and without any uniquely defining character". Geesink (1984: 4) admitted that "the contents of this chapter [Delimitations of Millettieae and related tribes] will be disappointing for those who expect a final answer to the questions suggested by the title". 
Geesink's major revision of this alliance, which he much enlarged to comprise 43 genera within Tribe Millettieae, was clearly polyphyletic with an assemblage of taxa having a range of unifying as well as contradicting characters. Although far from definitive, this revision was a major step forward and did lay a sound basis for subsequent research in the tribe. Geesink's (1984) generic treatment included a set of genera in the "Callerya Group" (Hu et al. 2002; Hu and Chang 2003): Wisteria Nutt. (1818), Callerya Endl. (1843), Afgekia Craib (1927) and Endosamara Geesink (1984). Subsequently, Sarcodum Lour. (1790) was also placed in this group (Schrire 2005; Clark 2008) (see Table 1). The genus Antheroporum Gagnep., placed tentatively in this grouping by Schrire (2005), has subsequently been shown to belong within the core-Millettieae (LPWG 2016; Mattapha 2017: 53).

Over the past 30 years a large number of DNA-based phylogenies have analysed many taxa from Tribe Millettieae (Palmer et al. 1987; Lavin et al. 1990; Doyle et al. 1997; Lavin et al. 1998; Doyle et al. 2000; Kajita et al. 2001; Hu et al. 2000; Hu et al. 2002; Hu and Chang 2003; Wojciechowski et al. 2004; Schrire 2005; Schrire et al. 2009; Wink 2013; Li et al. 2014; de Quieroz et al. 2015). Analysis of data from the phytochrome gene family PHY (Lavin et al. 1998) has shown that a core-Millettieae group is monophyletic and may be defined by the presence of pseudoracemes and pseudopanicles. Moreover, while the millettioid-phaseoloid alliance as a whole falls within the large non-protein amino acid accumulating (NPAAA) clade (Wojciechowski et al. 2004; Cardoso et al. 2012; 2013; Wink 2013; Wojciechowski 2013; De Quieroz et al. 2015), Lavin et al. (1998) showed that the core-Millettieae group are diagnosed by a loss of the ability to accumulate the non-protein amino acid canavanine. In these studies the Callerya group does not belong with the Millettioid group but rather is accommodated in the Hologalegina clade (Wojciechowski et al. 2000; Lavin et al. 2005). Lavin et al. (1998) also revealed that Afgekia, Callerya, Endosamara and Wisteria, i.e. a significant part of the Callerya group, did accumulate canavanine rather than alkaloids in their seeds and that they all possessed either true panicles or true racemes. Furthermore, Lavin et al. (1998) postulated that owing to the presence of true racemes, Sarcodum was also likely to accumulate canavanine and would therefore not be part of the core-Millettioid group. The morphological distinction between true and pseudoracemes is that in "true" racemes the flowers are inserted singly on the rachis (the unit comprising a flower, pedicel and bract). Pseudoracemes (Lackey 1981) on the other hand, consist of more than one flower inserted at a node on the rachis (the unit comprising two or more flowers, pedicels and bracts all subtended by a secondary bract representing branch reduction). Racemes and pseudoracemes are further compounded into panicles and pseudopanicles.

The Callerya group occurs in a more inclusive subset of taxa that all lack one copy of chloroplast DNA, the Inverted Repeat Lacking Clade or IRLC (Palmer et al. 1987; Lavin et al. 1990; Liston 1995; Doyle et al. 1997; Wojciechowski et al. 2000). The loss of a prominent inverted repeat structure in cpDNA in legumes had previously been observed in the genera Vicia (Koller and Delius 1980) and Pisum (Palmer and Thompson 1981). The genome of Wisteria was also discovered to have deleted one half 
of the inverted repeat amounting to $25 \mathrm{~kb}$ of DNA (Palmer et al. 1987). It was apparent that whereas the other legume genera were rearranged genetically as a result of the loss of the inverted repeat, both Wisteria floribunda and Medicago sativa remained otherwise unrearranged (Palmer et al. 1987). The IRLC is sister to Tribes Loteae, Sesbanieae and Robinieae (Lewis et al. 2005), which retain the inverted repeat (Lavin et al. 2005; Cardoso et al. 2012; Cardoso et al. 2013; LPWG 2013, 2017). Additional evidence from chloroplast $r b c L$ sequence data has also revealed that the Millettieae lie outside the IRLC (Lavin et al. 1990; Doyle et al. 1997; Kajita et al. 2001; Hu and Chang 2003), while the Callerya group all fall within the IRLC. These data refute the previously made assumptions that the group belongs with the Millettieae. Lavin et al. (1990) noted that although Wisteria and Millettia japonica both showed hypogeal seed germination and a lianescent habit, which are characteristic of many Millettieae genera, these species differed from the Millettieae in their wholly temperate distribution, the lack of the inverted repeat and both had a base chromosome number of $\mathrm{x}=8$ as opposed to $\mathrm{x}=11$ or 12 . The analyses of Hu and Chang (2003), based on plastid $r b c L$ sequence data, confirmed that Afgekia sericea, Callerya vasta, Endosamara racemosa, Millettia japonica and two Wisteria species all belonged within the large IRLC. Their results, however, were based on comparatively limited taxon sampling of taxa within the Callerya group.

Wink (2013) examined 1276 species of Leguminosae for the distribution of secondary metabolites mapped against phylogenetic trees generated by combined sequence data from cpDNA $r b c L$, matK and nrDNA ITS. In the study, it was shown that Wisteria and Callerya nested within the IRLC and that they possessed isoflavones in common with most but not all other taxa within the IRLC.

A unique marker further distinguishes the Callerya group, adding weight to the distinctiveness of this assemblage of genera. Jansen et al. (2008) undertook a comprehensive survey for the retention or loss of two chloroplast introns among 301 legume species representing three subfamilies and 198 genera. Their survey of the presence or absence of the rps 12 intron revealed that along with 49 of the millettioid-phaseoloids sampled from outside the IRLC, Afgekia filipes, A. sericea, Callerya atropurpurea, C. australis, C. megasperma, C. pilipes, Endosamara racemosa, Millettia (sic) japonica, Wisteria brachybotrys, W. floribunda, W. frutescens, W. macrostachya and W. sinensis - each from inside the IRLC - all retained the intron. Of the 77 other taxa sampled from within the IRLC all - without exception - had lost the intron. Significantly, therefore, all genera within the IRLC surveyed for the presence or absence of the $r p s 12$ intron showed it to be lacking, except for the Callerya group, marking out the latter as unique within the IRLC (Jansen et al. 2008). Seven species of Glycyrrhiza surveyed by Jansen et al. (2008), whose position in recent phylogenies (Doyle et al. 2000; Lewis et al. 2005; LPWG 2013, 2017; Li et al. 2014), was placed sister to the Callerya group within the IRLC, all lacked the rps12 intron. Glycyrrhiza, which is represented in our analyses, has therefore not been included as part of the Callerya group.

The Callerya group is thus uniquely diagnosed by a combination of lacking the 25 $\mathrm{kb}$. inverted repeat of cpDNA and possessing the cpDNA rps 12 intron. Representa- 


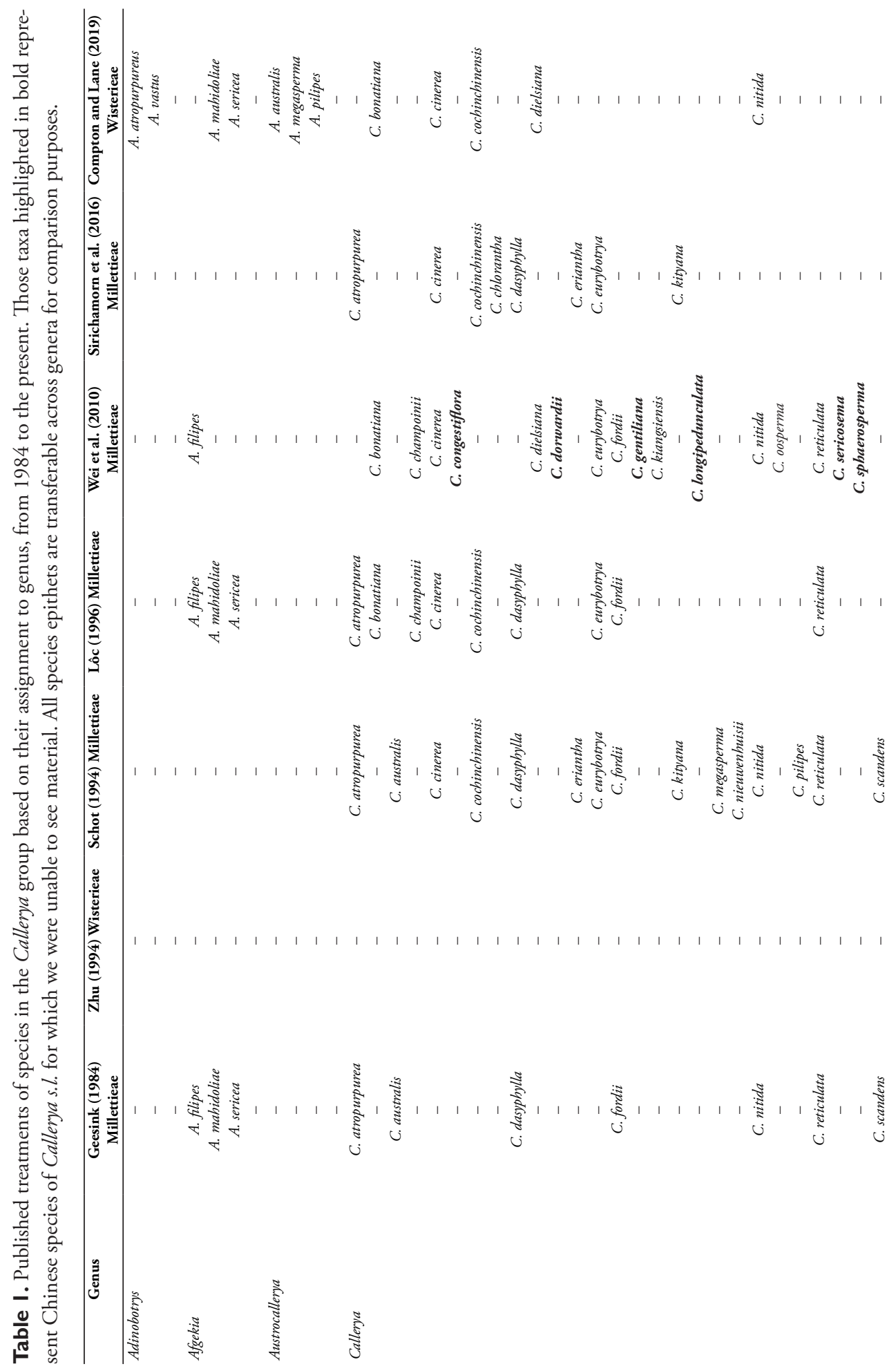


The Callerya Group redefined and Tribe Wisterieae (Fabaceae) emended based on...

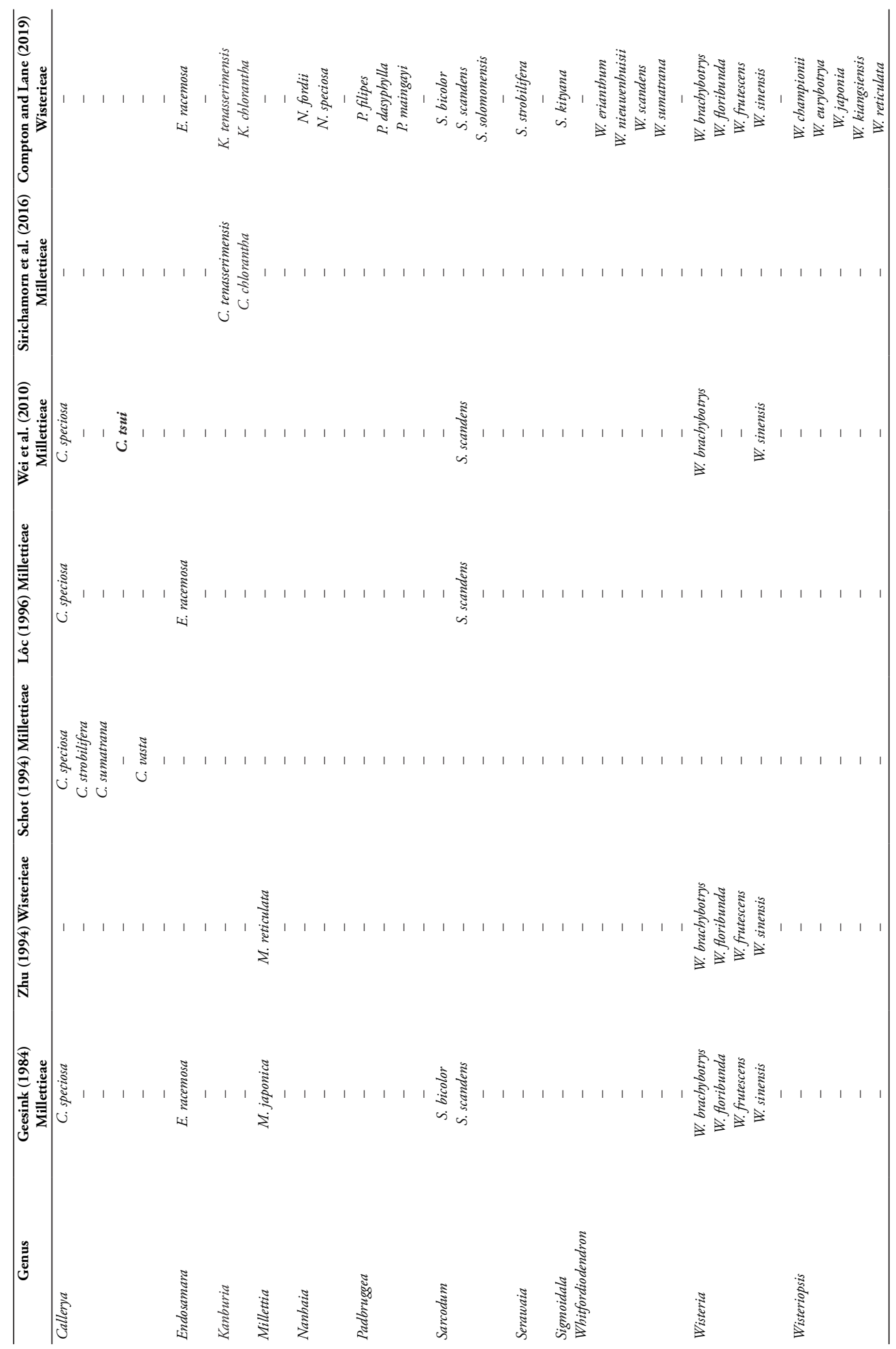


tives of Afgekia, Callerya and Wisteria from this subgroup of taxa have also been found to group together according to data from sequences of nuclear DNA ITS spacer regions (Hu et al. 2002; Li et al. 2014).

Zhu (1994) defined her new Tribe Wisterieae comparing only pollen from four species of Wisteria to the millettioid-phaseoloid genera Craspedolobium Harms, Derris, Millettia, Pongamia and Tephrosia Pers. The genus Pongamia Adans. is now considered synonymous with Millettia Wight \& Arn. (Schrire 2005: 383). The pollen grains of the four Wisteria species exhibited much broader polar regions (apocolpia) and a distinctive reticulate pollen surface compared to the other taxa examined. Zhu (1994) also made comparisons of Wisteria and other millettioid-phaseoloid genera using data from phytochemical and embryological analyses as well as noting the chromosome count of $2 \mathrm{n}=16$ in Wisteria compared to those of other genera whose members frequently have $2 \mathrm{n}=22$ or $2 \mathrm{n}=24$. It is notable that within the Callerya group, two species of Afgekia; A. sericea and $A$. mahidoliae also have chromosome counts of $2 \mathrm{n}=16$ (Prathepha 1994; Prathepha and Baimai 2003). Zhu's (1994) concept of Tribe Wisterieae thus was based solely on four samples of Wisteria and one accession of Millettia [Callerya] reticulata (Table 1).

One taxon recently recognised as belonging in the Callerya group was included under the names Wisteria japonica or Millettia japonica (Doyle et al. 1997; Doyle et al. 2000; Kajita et al. 2001; Hu and Chang 2003). The inclusion of this taxon in Wisteria was originally based on the deciduous leaves, twining habit, pendulous inflorescences and flowers where the wing petals are free from the keel (Siebold and Zuccarini 1839; Bailey and Bailey 1949; Geesink 1984; Iwatsuki et al. 2001; Compton and Thijsse 2013; Compton 2015c). Its summer flowering habit, paniculate inflorescences and absence of callosities on the standard petals have also been used to segregate it from Wisteria (Gray 1858; Dunn 1912a; Schot 1994; Valder 1995).

All genera currently comprising the Callerya group (Table 1): Afgekia, Callerya, Endosamara, Wisteria [incl. Millettia japonica)] and Sarcodum possess bracts enclosing the apical floral buds prior to anthesis and all bear either true racemes or true panicles. All are lianas with the exception of two tree species Callerya atropurpurea (Wall.) Schot and C. vasta (Kosterm.) Schot.

The genus Sarcodum was not included in the analysis of Jansen et al. (2008) and has not been sampled for DNA analysis prior to this paper but the generic morphological characters (Table 4) place it firmly within the Callerya group.

The genus Callerya Endl., the largest genus within the group with 33 species (Table 1) has subsequently been found to be polyphyletic (Li et al. 2014). Without a comprehensive morphological study of the genus and its near relations and in the absence of additional DNA evidence, it is fair to state that the genus has been something of a catch-all for taxa that bear some morphological affinities with each other (Schot 1994; Lôc 1996). The purpose of this paper therefore is to test generic boundaries within the Callerya group by reassessing morphological characters and by a comprehensive molecular sequencing investigation of representative species of all taxa within the group using both nuclear and chloroplast genes. 


\section{Materials and methods}

\section{Molecular preparation and sequencing}

Taxon sampling included those taxa in the DNA based phylogenies of Doyle et al. (1997), Hu et al. (2002), Hu and Chang (2003), Jansen et al. (2008) and Li et al. (2014 - but not including several species they recognised in the Callerya cinerea complex). Three chloroplast regions were included in the study. Two protein coding genes: mat $K$ and $r b c L$, and the intergenic spacer $n d h J-t r n F$. One nuclear gene region was also included in the study ITS1, 5.8S and ITS2. Fresh DNA was extracted from the previously unsampled Sarcodum scandens (Tables 1, 2). For the ITS dataset, 12 additional sequences representing the Callerya group and 26 outgroup sequences were included from GenBank; the matK dataset comprised an additional five Callerya group sequences and 17 outgroup sequences from GenBank and the $r b c L$ dataset, a further two ingroup and 12 outgroup sequences from GenBank (Table 2). Millettia japonica has also been confused with Wisteria floribunda in DNA sampling (see GenBank KT119544) and as a result of this, we have chosen to include three different verified samples in this study in order to ascertain its placement within the Callerya group.

Outgroup taxa (Table 2) for each analysis comprised several accessions that represented taxa from other Tribes within the IRLC e.g. Hedysareae, Galegeae and Trifolieae (Lewis et al. 2005) and several from outside the IRLC, e.g. Robineae, Loteae, Sesbaneae, Millettieae, Abreae, Phaseoleae and Indigofereae. The genus Schfflerodendron Harms was selected as the outgroup upon which to root all trees owing to its position in the LPWG (2017) RAxML Maximum Likelihood analysis. Its position as sister to Callerya atropurpurea - and these two to Glycyrrhiza - in turn all sister to the Callerya group and the rest of the IRLC suggest that it is the most appropriate candidate for choice as outgroup. Additional outgroup sequences were generated of W095 (see codes to samples, Table 2) Schefflerodendron usambarense (Tribe Millettieae), W113 Lotus uliginosus (Tribe Loteae) and W115 Austrosteenisia glabristyla (Tribe Millettieae) with the addition of 14 other legume genera from GenBank representing additional Tribes all of which sit outside the IRLC (Table 2).

We generated 49 sequences of the nrDNA ITS spacer region, including one for the outgroup taxon Schefflerodendron usambarense. Sequence data was also generated for three plastid markers: 51 Callerya group sequences from the $n d h J-t r n F$ cpDNA intergenic spacer, 53 sequences from the matK gene and 57 sequences from the $r b c L$ gene. Sequences of three outgroup taxa (i.e. Austrosteenisia glabristyla, Lotus uliginosus and Schefflerodendron usambarense) were also obtained for these three plastid markers (see Table 2). Summary statistics of support levels at critical nodes of all trees generated in this study (Suppl. material 1 Figs S1-S6), derived from Maximum Likelihood (ML) analysis and Bayesian inference (BI) analysis, are shown in Table 3.

The DNA extraction protocol for all 54 samples (with numbers from W002 to W115) and the seven samples labelled W1, W2, W3, W5, W6, W8 and W10 
(Table 2) used a modified CTAB protocol (Doyle and Doyle 1987). DNA extraction from herbarium specimens followed the protocol used by Särkinen et al. (2012) with some minor amendments.

For all accessions labelled W002 to W115 (Table 2), a circa 800 bp part of matK was amplified with the previously unpublished primers designed by Ki-Joong Kim: 1RKIM-f - ACCCAGTCCATCTGGAAATCTTGGTTC and 3FKIM-r - CGTACAGTACTTTTGTGTTTACGAG (Dunning and Savolainen 2010). PCR reactions were performed in $25 \mu \mathrm{l}$ volumes containing final concentrations of $1 \times$ Bioline Biomix Red, $0.35 \mu \mathrm{M}$ of each primer, $0.2 \mathrm{mg} / \mathrm{ml}$ BSA (bovine serum albumin), $4 \% \mathrm{v} / \mathrm{v}$ DMSO (dimethyl sulfoxide) and 20ng DNA template. Cycling conditions were $94^{\circ} \mathrm{C}$ for 120 s, then 35 cycles of $94^{\circ} \mathrm{C}$ for 30 s, $48^{\circ} \mathrm{C}$ for 30 s, $72^{\circ} \mathrm{C}$ for 60 s, and finally $72^{\circ} \mathrm{C}$ for 7 minutes.

For all accessions (Table 2) the gene $r b c L$ was amplified with primers $1 \mathrm{~F}$ (Fay et al. 1997) and 1460R (Fay et al. 1998). To amplify degraded and/or low quality DNA two overlapping shorter fragments were amplified with the original primers and internal primers 636F and 724R (Fay et al. 1997). PCR reactions for all primer combinations were performed in $25 \mu$ l volumes containing final concentrations of $1 \times$ Bioline Biomix Red, $0.35 \mu \mathrm{M}$ of each primer, $0.2 \mathrm{mg} / \mathrm{ml}$ BSA (bovine serum albumin), and 20ng DNA template. Cycling conditions for the reactions using primers $1 \mathrm{~F}$ and $1460 \mathrm{R}$ were $94^{\circ} \mathrm{C}$ for 120 s, then 30 cycles of $94^{\circ} \mathrm{C}$ for $60 \mathrm{~s}, 51{ }^{\circ} \mathrm{C}$ for $30 \mathrm{~s}, 72{ }^{\circ} \mathrm{C}$ for 120 s, and finally $72{ }^{\circ} \mathrm{C}$ for 7 minutes. For the shorter fragments this protocol was modified by decreasing the elongation time to $90 \mathrm{~s}$ and increasing the number of cycles to 40 for weaker amplicons.

Again for all accessions, the intergenic spacer $n d h J-t r n F$ was amplified with the primers $n d h J$ and TabE using the PCR protocol listed in Shaw et al. (2007). Low quality or degraded DNA necessitated the utilisation of primers that amplified two shorter, overlapping segments of this region. We designed two additional primers internal to the $n d h J-t r n F$ spacer in order to overcome this problem: 456F - ATGGGCCGGATTCTATTTGT and 725R - TGATTAGTGGTCTAGATCATCAT. The PCR protocol for the shorter fragments was the same as above, apart from increasing the number of cycles to 40 for weaker amplicons.

For all accessions the nrDNA Internal Transcribed Spacers (ITS1 and ITS2) were amplified with primers ITS4 and ITS5 (White et al. 1990; Baldwin et al. 1995) or with 17SE and 26SE (Sun et al. 1994). The PCR reactions for ITS4 and ITS5 were performed in $25 \mu \mathrm{l}$ volumes containing final concentrations of $1 \times$ Bioline Biomix Red, $0.5 \mu \mathrm{M}$ of each primer, $0.2 \mathrm{mg} / \mathrm{ml}$ BSA (bovine serum albumin), and $10-25 \mathrm{ng}$ DNA template. Cycling conditions were $94^{\circ} \mathrm{C}$ for 120 s, then 30 cycles of $94^{\circ} \mathrm{C}$ for 60 s, $48{ }^{\circ} \mathrm{C}$ for $60 \mathrm{~s}, 72{ }^{\circ} \mathrm{C}$ for $90 \mathrm{~s}$, and finally $72{ }^{\circ} \mathrm{C}$ for 7 minutes. The PCR reactions for $17 \mathrm{SE}$ and 26SE were performed in $25 \mu \mathrm{l}$ volumes containing final concentrations of $1 \times$ Bioline Biomix Red, $0.35 \mu \mathrm{M}$ of each primer, $0.2 \mathrm{mg} / \mathrm{ml}$ BSA (bovine serum albumin), and 20ng DNA template. Cycling conditions were $94^{\circ} \mathrm{C}$ for 120 s, then 40 cycles of $94{ }^{\circ} \mathrm{C}$ for 30 s, $63{ }^{\circ} \mathrm{C}$ for $60 \mathrm{~s}, 72{ }^{\circ} \mathrm{C}$ for 60 s, and finally $72{ }^{\circ} \mathrm{C}$ for 7 minutes.

Sequencing of 44 taxa for ITS and 54 taxa for $n d h J-\operatorname{trn} F$, mat $K$ and $r b c L$ were performed at GATC Biotech (www.gatc-biotech.com; Konstanz, Germany). 


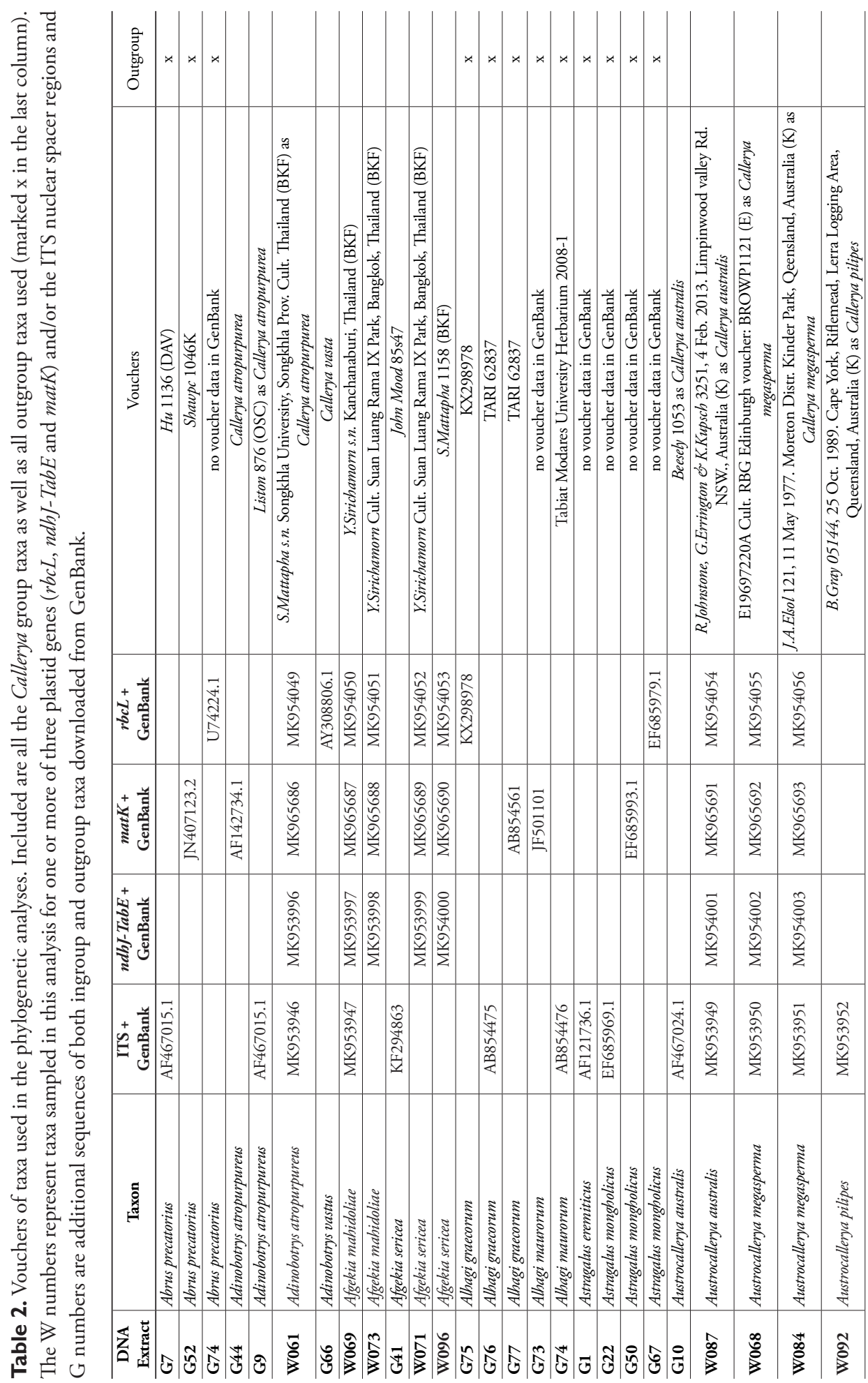




\begin{tabular}{|c|c|c|c|c|c|c|c|c|c|c|c|c|c|c|c|c|c|c|c|c|c|c|c|}
\hline $\begin{array}{l}\text { 言 } \\
\text { 总 } \\
\bar{z}\end{array}$ & & $x$ & & & & & & & & & 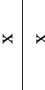 & $x$ & $\star$ & $4 x$ & \begin{tabular}{l|l}
$*$ & $\star$
\end{tabular} & & & & \begin{tabular}{|l|l}
$*$ & $*$
\end{tabular} & & $\star \star x$ & $\star \star \star$ & $x$ \\
\hline 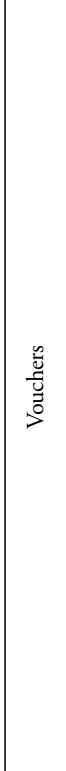 & 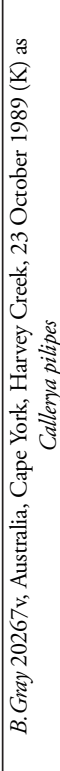 & 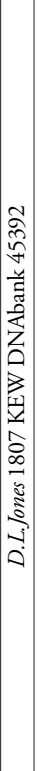 & 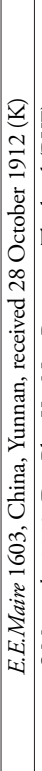 & 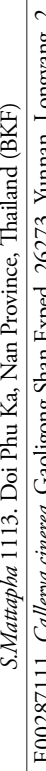 & 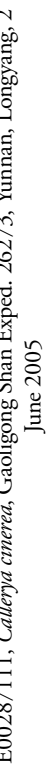 & 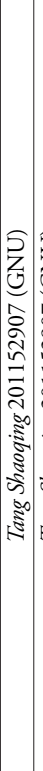 & 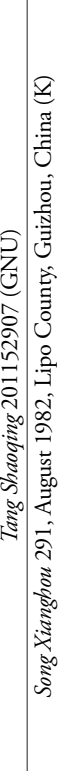 & 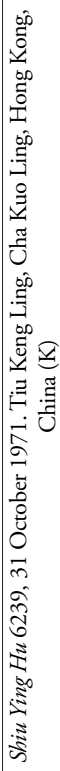 & 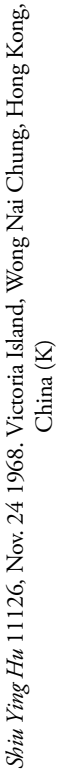 & 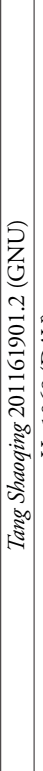 & 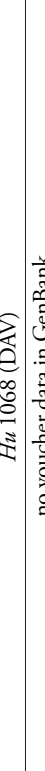 & 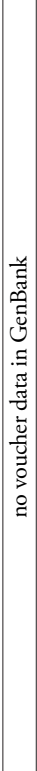 & 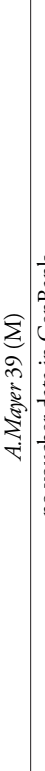 & 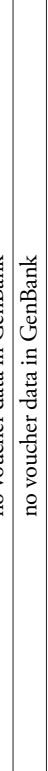 & 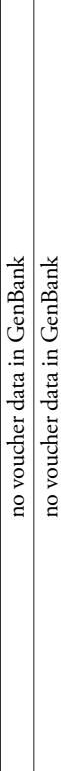 & 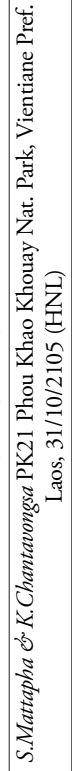 & 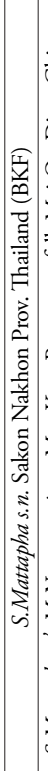 & 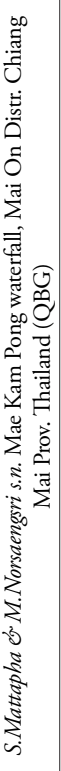 & 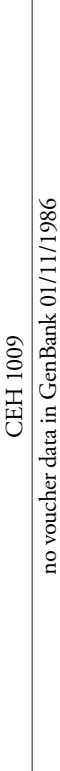 & 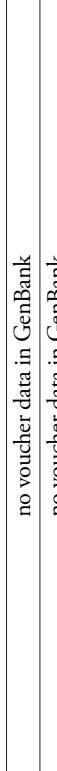 & 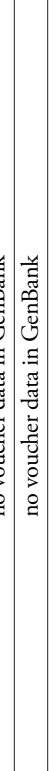 & 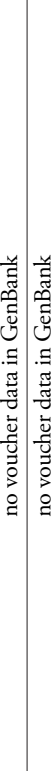 & 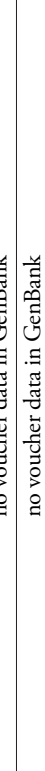 \\
\hline 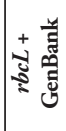 & & 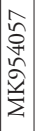 & 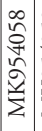 & 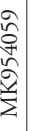 & 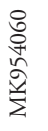 & & $\begin{array}{l}\vec{\delta} \\
\bar{d} \\
\hat{2} \\
\hat{y} \\
\Sigma\end{array}$ & 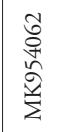 & 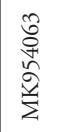 & & $\widetilde{7}$ & & & & & 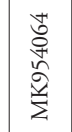 & 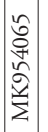 & 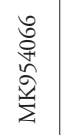 & & & & 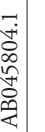 & \\
\hline 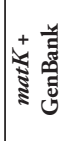 & & 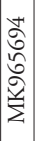 & $\begin{array}{l}\hat{n} \\
\hat{\sigma} \\
0 \\
2 \\
\dot{v} \\
\Sigma\end{array}$ & & 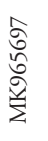 & & 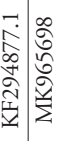 & 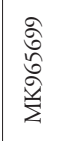 & 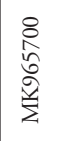 & & & & 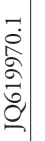 & 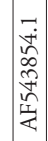 & & $\begin{array}{l}\overline{2} \\
\hat{n} \\
\ddot{\delta} \\
\dot{z}\end{array}$ & 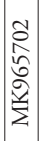 & 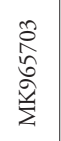 & & 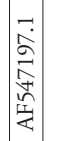 & & & \\
\hline 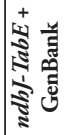 & & 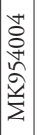 & & 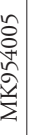 & 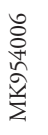 & & 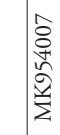 & 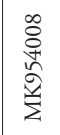 & 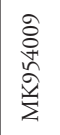 & & & & & & & 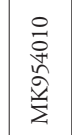 & $\begin{array}{l}\bar{z} \\
\bar{y} \\
\hat{u} \\
\hat{2} \\
\dot{z}\end{array}$ & $\begin{array}{l}\tilde{a} \\
\bar{\sigma} \\
\tilde{v} \\
\tilde{z} \\
\Sigma\end{array}$ & & & & & \\
\hline 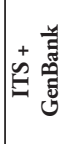 & $\begin{array}{l}\hat{\tilde{\hat{n}}} \\
\hat{\tilde{\varepsilon}} \\
\tilde{\Sigma}\end{array}$ & 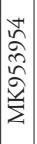 & $=$ & $\Xi$ & 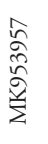 & ָิ & 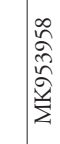 & 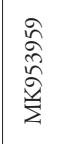 & $\begin{array}{l}\stackrel{8}{\delta} \\
\hat{\alpha} \\
\hat{\tilde{z}}\end{array}$ & & 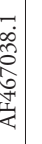 & $\frac{\overbrace{0}^{\circ}}{0}$ & & & 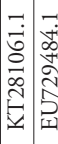 & & $\mid \begin{array}{l}\vec{\Xi} \\
\widehat{\tilde{2}} \\
\hat{\tilde{z}} \\
\hat{\Sigma}\end{array}$ & 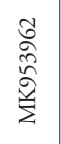 & 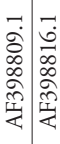 & & $\overrightarrow{0}$ & & \\
\hline
\end{tabular}

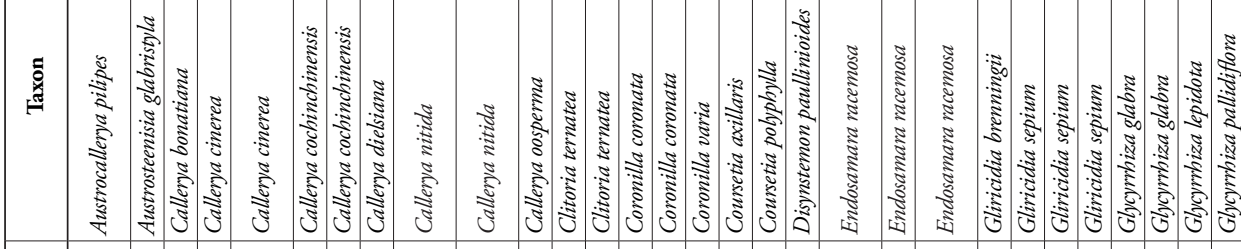

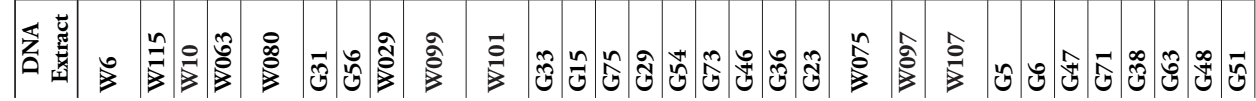




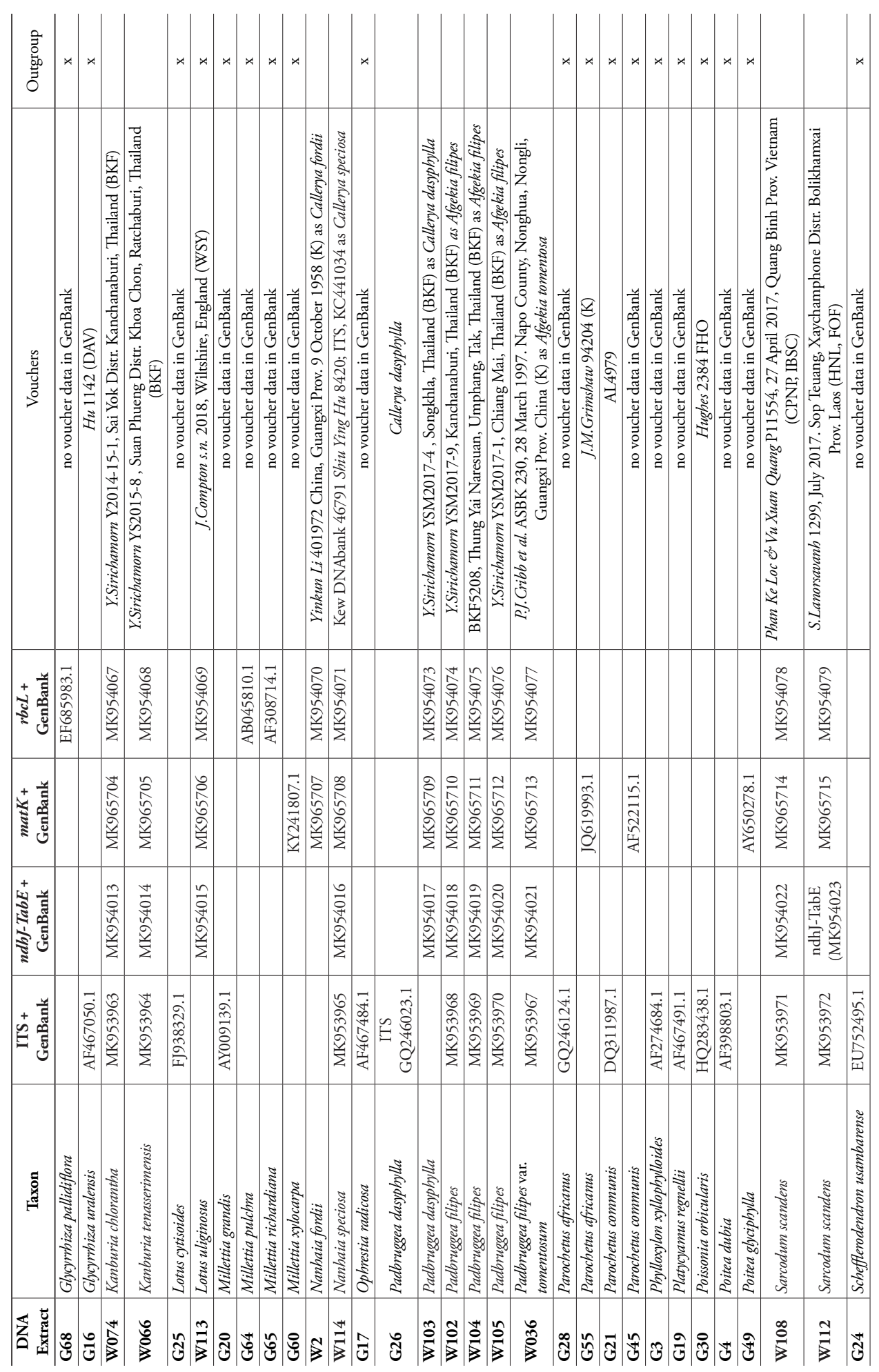




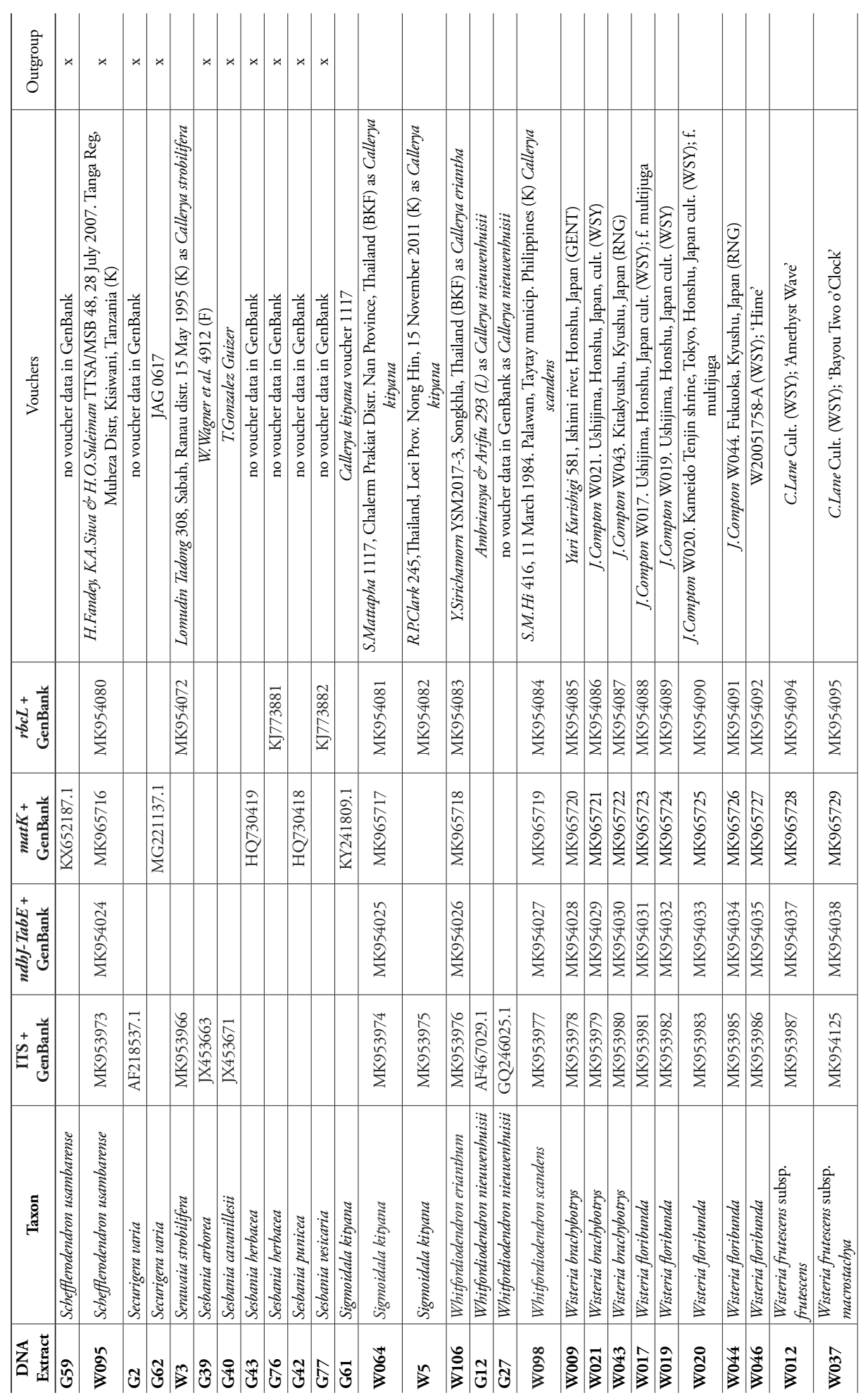




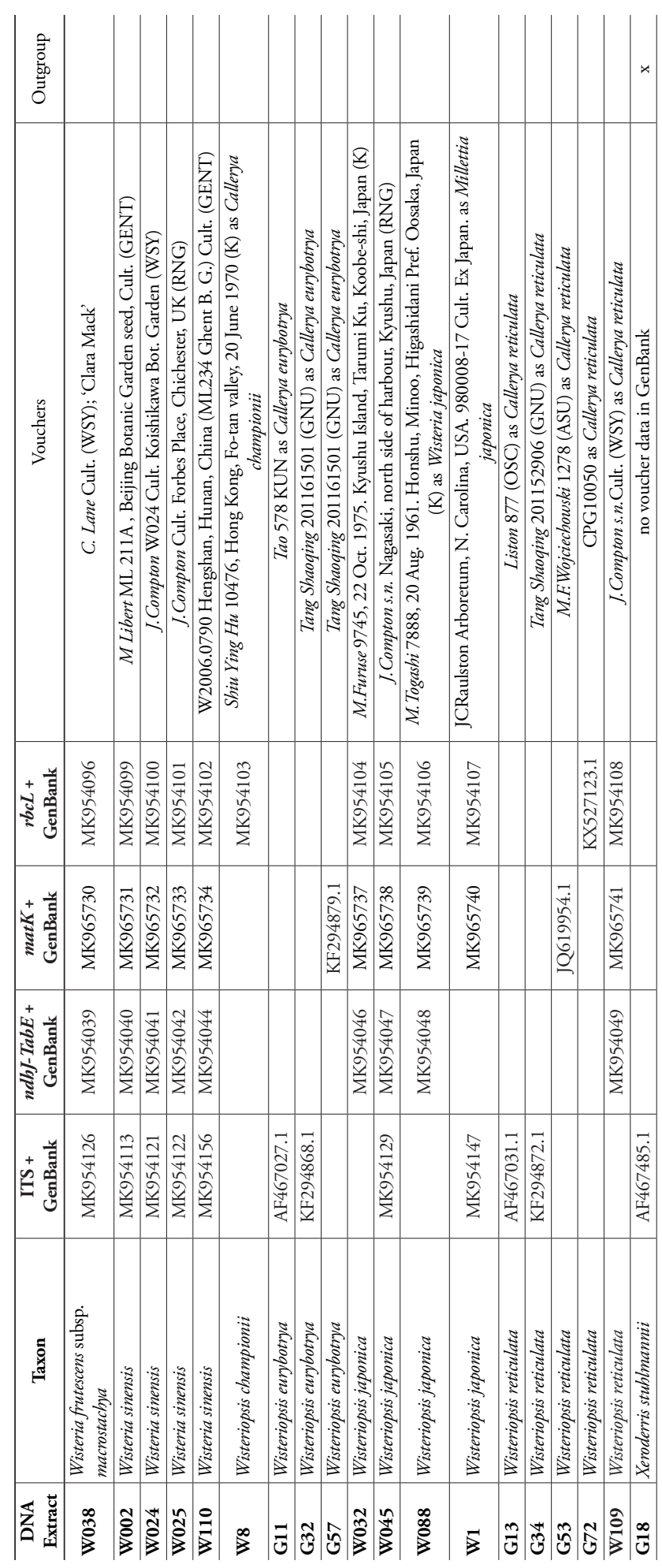


For the seven accessions labelled W1 to W10 (see Table 2) DNA extractions used a similar protocol to that mentioned above. PCR amplifications were performed using the same primers as already mentioned for nrDNA ITS, cpDNA matK and cpDNA $r b c L$ with the following different protocol: PCRs were performed in $25 \mu \mathrm{L}$ volumes, containing 12.5 $\mu \mathrm{L}$ DreamTaq PCR Master Mix $(2 \times)(4 \mathrm{mM} \mathrm{MgCl}$; Thermo Fisher Scientific, Waltham, MA, USA), $0.5 \mu \mathrm{L}$ of each primer (100 ng $\mu \mathrm{L}-1)$, and $1 \mu \mathrm{L}$ DNA template. TBT-PAR [trehalose, bovine serum albumin (BSA) and polysorbate-20 (Tween-20)] was added to reduce the inhibitory effects of polysaccharide and phenolic compounds (Samarakoon et al. 2013).

For the accessions W1 to W10 (Table 2) all amplifications were performed on a 9700 GeneAmp thermocycler (ABI, Warrington, UK). All PCR products were purified with either the QIAquick PCR kit (Qiagen, Hilden, Germany) or the Nucleospin Extract II kit (Machery-Nagel, Düren, Germany), following the manufacturers' protocols. Cycle sequencing reactions were performed in $5 \mu \mathrm{L}$ reactions using $0.5 \mu \mathrm{L}$ BigDye Terminator cycle sequencing chemistry (v3.1; ABI) and the same primers as for PCR. Complementary strands were sequenced on an ABI3730 automated sequencer.

\section{Phylogenetic procedures and analyses}

Sequences of each region were edited and compiled in Geneious (version 8.1.9; Kearse et al. 2012) and aligned with the MUSCLE algorithm (Edgar 2004) implemented in AliView (Larsson 2014). The ends of the alignments were trimmed to the point where all sequences were present and base calls were unambiguous.

Phylogenetic analyses were conducted on the plastid, ITS and combined plastid/ ITS matrices using two approaches, Maximum likelihood (ML) and Bayesian inference (BI). For the ML approach, we used the software RAxML (v. 8.2.8; Stamatakis 2014) as implemented on the CIPRES portal (www.phylo.org) with 1,000 rapid bootstrap replicates followed by the search of the best ML tree; the GTRCAT model was used and all the other parameters were set as default settings. The Bayesian Markov Chain Monte Carlo (MCMC) approach was performed using the software MrBayes (version 3.2.6; Ronquist and Huelsenbeck 2003) as implemented on the CIPRES portal. The best-fit DNA substitution model was tested using JModelTest 2 (version 2.1.6; Darriba et al. 2012) as implemented on the CIPRES portal. The General Time Reversible (GTR) model with a proportion of invariable sites and a gamma shape to account for rate heterogeneity among sites $(G T R+I+G)$ was selected for both partitions. The analyses were run twice each for 10 million generations and sampled every $1000^{\text {th }}$ generation. The MCMC sampling was verified using Tracer (Rambaut and Drummond 2009) and was considered adequate when the effective sampling size was higher than 200. A burn-in period of one million generations was applied to each run. The remaining trees from both runs were compiled using the "allcompat" option in MrBayes to produce a maximum credibility tree with Bayesian posterior probabilities (BPP) for 
each node. In both combined ML and BI analyses, the plastid and ITS partitions were allowed to have partition-specific model parameters. Schefflerodendron usambarense was designated as outgroup taxon in all analyses. Support values for nodes of critical taxa in the Discussion are shown in Table 3.

\section{Morphological study}

The morphological key to the species was based on examination of living material in cultivation in UK and USA and in the wild in China, Japan, Laos, Myanmar, Thailand and Vietnam. Herbarium specimens were examined including the collection of all relevant genera in the Callerya group at $\mathrm{K}$ and BM. Online collections were examined at the Chinese Virtual Herbarium, CVH (http://www.cvh.ac.cn/en); JSTOR Global Plants (https://plants.jstor.org/); Herbarium, Muséum National d'Histoire Naturelle, Paris, MNHN (https://science.mnhn.fr/institution/mnhn/collection/p/item/search/ form?lang=en_US); Herbarium Royal Botanic Garden, Edinburgh, RBGE (http://data. rbge.org.uk/search/herbarium/) and Nederlandse Natuurhistorische Collecties, Naturalis (http://bioportal.naturalis.nl/). See Appendix 1 for a full list of all specimens used as the basis for the new generic descriptions. Herbarium acronyms follow Thiers (2019, http:// sweetgum.nybg.org/science/ih/). A full list of all Herbaria cited is found in the acknowledgements. A list of the critical characters measured for this study is shown in Tables 4, 5.

\section{Results}

New diagnoses (emended where necessary) - and full descriptions - are given for all genera in the taxonomic treatment section, because nearly all established genera have been modified over various historical treatments to include and/or exclude species such that their present concepts are often significantly different from the original protologue. Keys to genera and to all species (excluding those Chinese taxa of Callerya s.str. that we were unable to access) and extensive synonymy and typifications are also provided.

The combined analyses are consistent with respect to their ingroup topologies and are combined in the reference phylogenetic tree of this paper, Fig. 1 (see also Suppl. material 1: Figs S1, S2). In the plastid analyses (Suppl. material 1: Figs S3, S4) the main difference to Fig. 1 is Serawaia grouping with Clades D + E with no support in the $\mathrm{BI}$ analysis - and with Clades $\mathrm{C}+\mathrm{D}+\mathrm{E}$, with no support, in the ML analysis. The ITS BI analysis is similar to Fig. 1 except for the merging of Clades B $+C$. In the ITS $\mathrm{ML}$ analysis Clade A also breaks down together with Clades $\mathrm{B}+\mathrm{C}$ as Endosamara is attracted into Clade $\mathrm{C}$ from Clade $\mathrm{A}$. As an indication of variability across the four genes, the average percentage identity over the alignments (i.e., pairwise percentage of identity) is: plastid vs. ITS analyses $(92.5 \%, 77.1 \%)$ and within the plastid analyses, matK, $r b c L$ and $n d h J-\operatorname{trn} F(95.4 \%, 96.9 \%$ and $86.6 \%)$. 


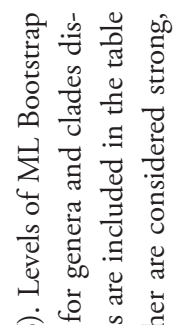

के

क 窝 节

50 त

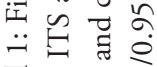

펼 च

芚冬岕

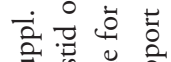

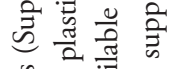

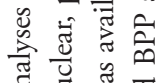

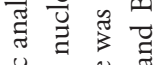

氙 चี

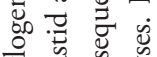

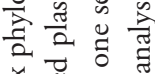

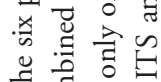

छ है

घ ठํ ठ

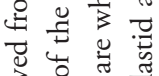

:

४ ष

过苍苛

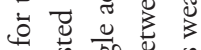
$\stackrel{0}{=} \cong$

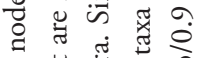

चु

:

के है है

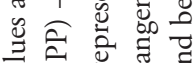

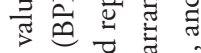

등

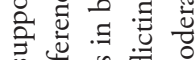

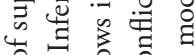

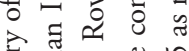

言芯芯苍令 हี

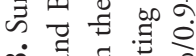

m

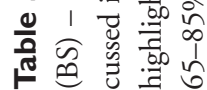

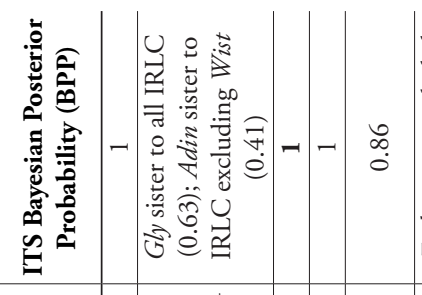

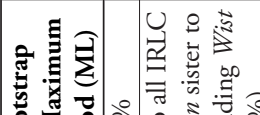

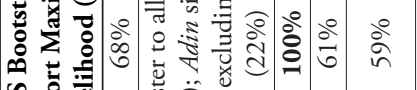

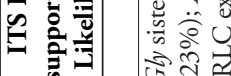

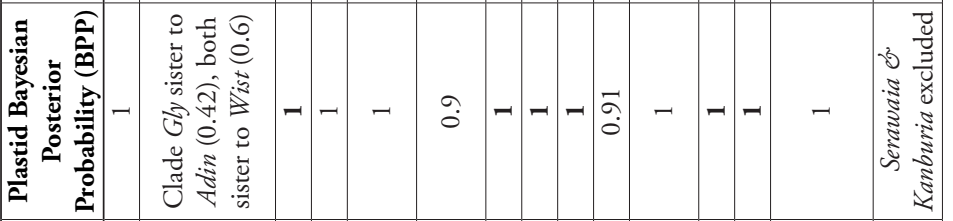

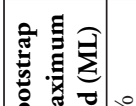

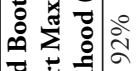

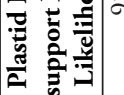

$\underset{\tilde{T}}{\tilde{U}}$

豙

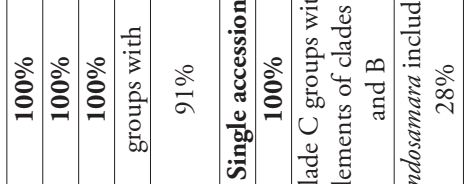

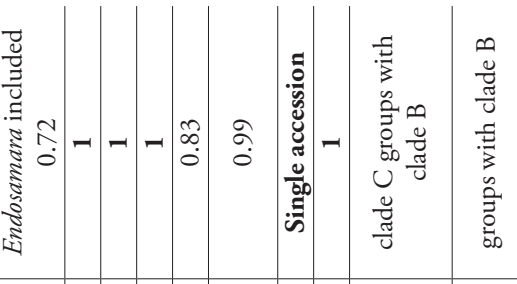

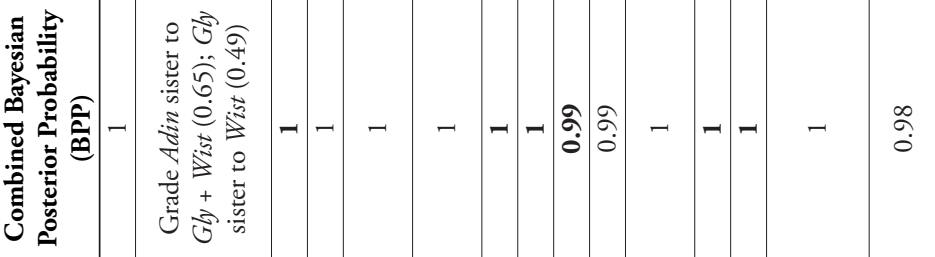

\section{害言最}

至
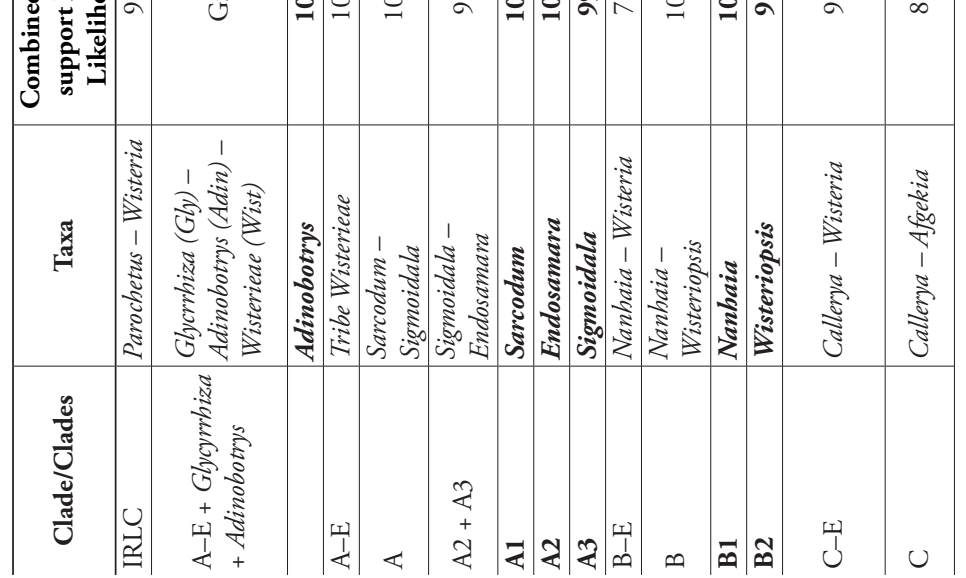
The Callerya Group redefined and Tribe Wisterieae (Fabaceae) emended based on...

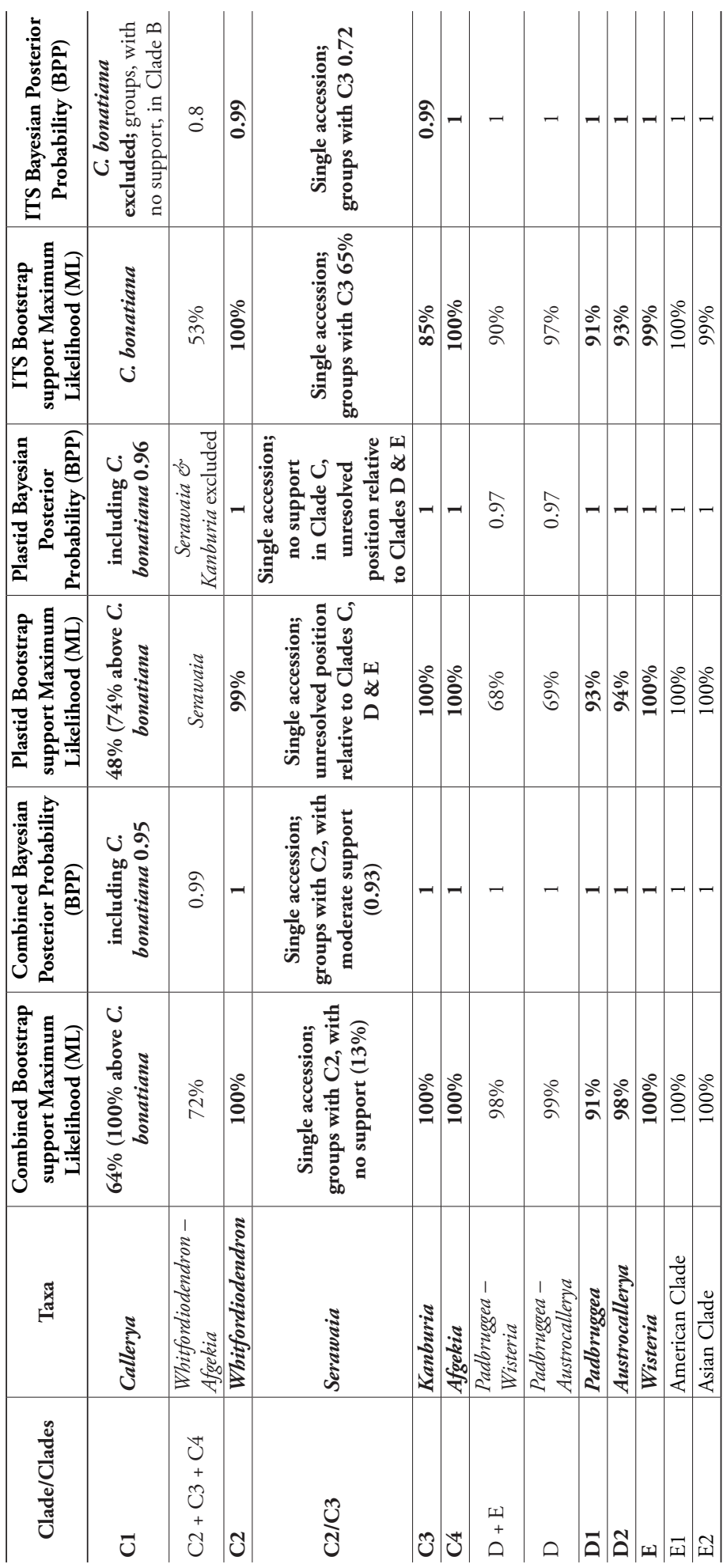




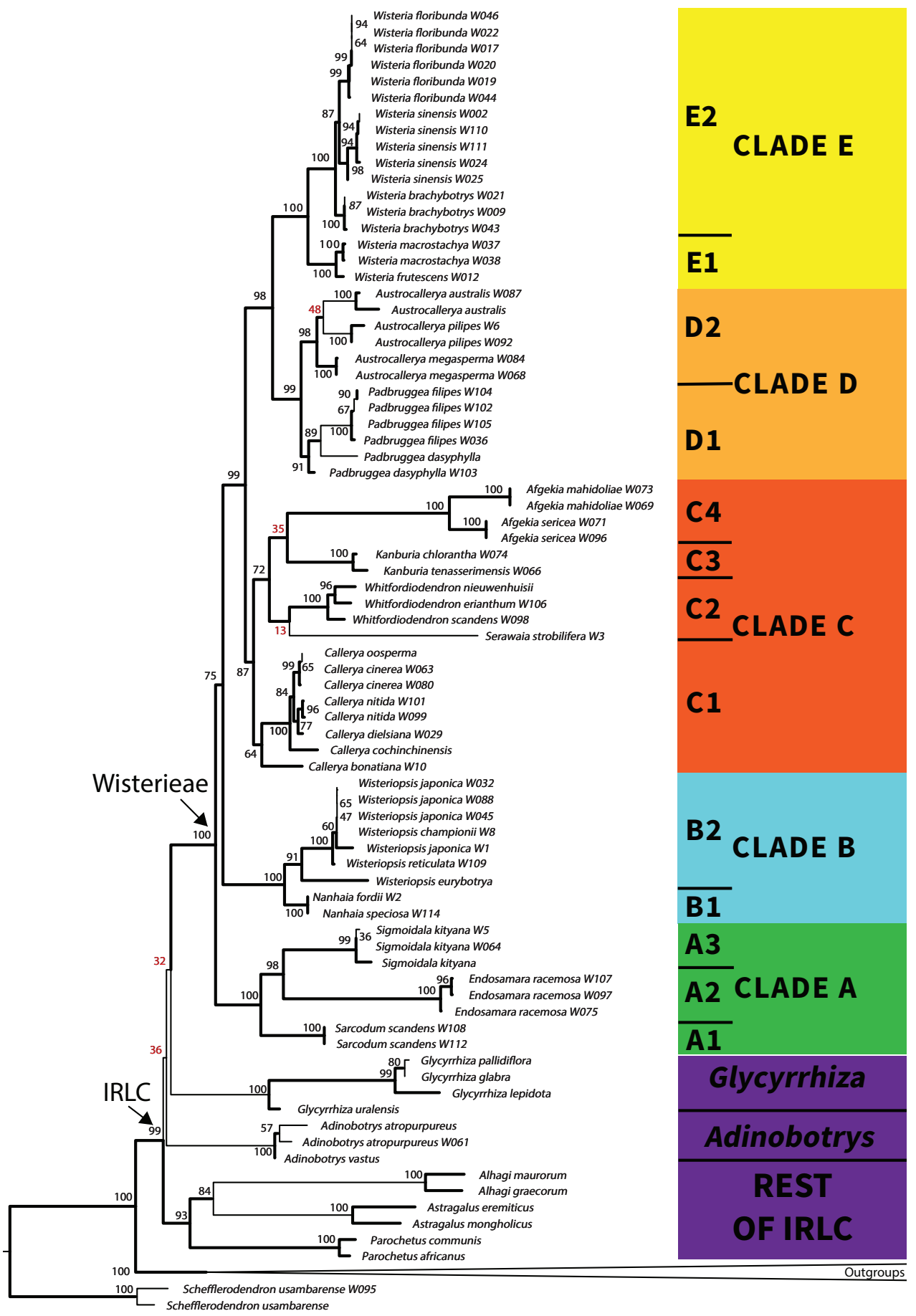


The Callerya group sensu Schot (1994); Lôc and Vidal (2001); Wei and Pedley (2010) and Sirichamorn et al. (2016) is not supported in its entirety in our analyses but rather, what emerges are four elements comprising the IRLC, each fully supported here (Fig. 1; Table 3), i.e. Tribe Wisterieae, Glycyrrhiza, Adinobotrys and a clade containing the Galegeae s.l., Hedysareae, Cicereae, Fabeae and Trifolieae s.l. (henceforth the "Temperate Tribe block"). Glycyrrhiza (Bootstrap or BS 100\%; Bayesian Posterior Probability or BPP 1) is not supported as sister to Tribe Wisterieae and neither is Adinobotrys (BS 100\%; BPP 1), which, in addition, is not supported to have a position within Tribe Wisterieae either. Adinobotrys is thus reinstated as a genus here and removed from the Callerya group. The positions of Tribe Wisterieae, Glycyrrhiza and Adinobotrys remain equivocal as regards their sister group relationships to the rest of the Temperate Tribe block of the IRLC.

The Callerya group i.e. Tribe Wisterieae without the genus Adinobotrys comprises five strongly supported clades with the first two in a basal grade leading to Clades $\mathrm{C}+$ $\mathrm{D}+\mathrm{E}$. The crown node of the tribe is fully supported in both the combined and plastid analyses (BS 100\%; BPP 1) and in the ITS BI analysis (BPP 1), although only weakly so in the ITS ML analysis (Fig. 1, Table 3, BS 61\%). Clade A (fully supported in the combined and plastid analyses but weakly so in the ITS analyses [BS 59\%, BPP 0.86]) contains the genus Sarcodum (BS 100\%; BPP 1), sequenced and analysed for the first time here, which is sister to Endosamara (BS 100\%; BPP 1) and the new monospecific genus Sigmoidala (BS 99\%; BPP 0.99) described in this paper. Clade B comprises two new genera described here, Nanhaia (BS 100\%; BPP 1) and Wisteriopsis (BS 91\%; BPP 1) and both are fully supported as Clade B (Fig. 1; Table 3). Our results confirm that the incorrectly attributed Millettia japonica is strongly supported within our new genus Wisteriopsis.

Figure I. Combined Maximum Liklihood (ML) and Bayesian Inference (BI) Phylogenetic tree of Tribe Wisterieae. The tree is derived from the combined plastid and ITS, RAxML bipartitions analysis representing 77 (36) ingroup samples (taxa) and 59 (40) outgroup samples (taxa). The outgroup Schefferodendron is used to root the trees. Lines in bold on the phylogeny incorporate results from the combined Bayesian Inference analysis, demarcating clades with BPP (0.95) support and above. Nodes are marked up with bootstrap values as percentages derived from the combined ML analysis with values of $50 \%$ or less marked in red. The collapsed portion of the tree, below the IRLC and above Schefflerodendron, represents the following genera (see Suppl. material 1: Figs S1-S6): Tribe Robinieae (Coursetia, Gliricidia, Poissonia \& Poitea); Tribe Sesbanieae (Sesbania), Tribe Loteae (Coronilla, Lotus \& Securigera); Tribe Millettieae (Millettia), Tribe Abreae (Abrus); Tribe Phaseoleae (Clitoria \& Ophrestia); Tribe Indigofereae (Phylloxylon) and basal millettioids (Austrosteenisia, Disynstemon, Xeroderris \& Platycyamus). Tribe Wisterieae is treated within five clades (Clades A-E), colour coded green for Clade A (Sarcodum, Endosamara \& Sigmoidala); cyan for Clade B (Nanhaia \& Wisteriopsis), red for Clade C (Callerya, Serawaia, Whitfordiodendron, Kanburia \& Afgekia); orange for Clade D (Padbruggea \& Austrocallerya) and yellow for Clade E (Wisteria). Each clade is further subdivided to represent the genera (except for the single accession of Serawaia which is incorporated with Whitfordiodendron in Clade C2) and E1 and E2 represent the geographical disjunction of species in Wisteria. Outgroups within the IRLC in purple include Glycyrrhiza, Adinobotrys and representatives of the Temperate Tribe block. The ingroup (IRLC) and Tribe Wisterieae are demarcated with arrows on the tree. 
Clade C is strongly supported (BS 87\%; BPP 0.98) in the combined analyses but is more labile with some genera excluded and others included in the plastid and ITS analyses (Fig. 1; Table 3). A much reduced Callerya s.str. together with C. bonatiana is strongly supported in the combined and plastid BI analyses (BPP 0.95; 0.96), but only weakly so in the ML analyses. The single accession of $C$. bonatiana has no support for grouping with Callerya in the ITS analyses. Callerya above $C$. bonatiana is strongly supported in the combined ML and BI analyses (BS 100\%; BPP 0.95). The grouping of Afgekia (BS 100\%; BPP 1), the resurrected genus Whitfordiodendron (BS 100\%; BPP 1) and the two new genera described here, Kanburia (BS 100\%; BPP 1) and Serawaia (single accession), is moderately supported in the combined ML (BS 72\%) and well supported in the combined BI (BPP 0.99) analyses. This grouping breaks down in the plastid analyses and is weakly supported in the ITS analyses (Table 3). Serawaia is strongly supported within Clade C in the combined analyses (BS 87\%; BPP 0.98) in a position (Fig. 1, Table 3), with no support, as sister to Whitfordiodendron. It is in an unresolved position in the plastid analyses and is weakly supported as sister to Kanburia in the ITS analyses (Table 3).

Clade D comprises two genera, Padbruggea (BS 91\%; BPP 1) which is reinstated as a genus here and Austrocallerya (BS 98\%; BPP 1), a new genus described here. Our results reveal that Afgekia filipes belongs in our reinstated genus Padbruggea and the transfer back is made in this paper. The two genera are also strongly supported together as Clade D (BS 98\%; BPP 1). Clades D + E are strongly supported in all analyses (combined ML [98\%] \& BPP [1]; plastid BPP [0.97] and ITS ML [90\%] \& BPP [1]), but in the plastid ML analysis support is weak (BS 68\%). Finally Clade E comprises Wisteria (BS 100\%; BPP 1), with two North American taxa fully supported as sister to the three Asian species of the genus (BS 100\%; BPP 1). The relationship of W. brachybotrys as sister to W. floribunda and W. sinensis is also fully supported (BS 100\%; BPP 1).

\section{Discussion}

\section{Morphology of the Callerya Group}

Schot (1994) segregated her species of Callerya into two groups based on the presence or absence of stipels and, when present, whether they were persistent or caducous. We have found no evidence that stipel presence or absence has any taxonomic significance in Calleya s.l. Lôc (1996) and Wei and Pedley (2010) segregated species on the basis of the presence or absence of an indumentum on the dorsal surface of standard petals. We concur with Lôc (1996) and Wei and Pedley (2010) but in addition regard the inflorescence type and various floral, fruit and seed types to be equally significant in delimiting taxa (see the Key to the Genera and Table 4 for a list of significant characters). The key to fruiting specimens of Callerya s.l. (Lôc 1996: 56) emphasised stipellae characters as distinctive, an observation for which we find no support. 
Using our revised generic concepts and species assigned to them (Table 1) and comparison of morphological characters (Table 4), the synapomorphies diagnosing the Callerya group are: the lianescent habit (except for the tree species Adinobotrys atropurpureus and $A$. vastus); flowers inserted singly on the axis in either axillary or terminal racemes and/or panicles, and bracts either fully or largely enclosing the flower buds at the inflorescence apex, which are usually longer and often wider than the buds. There are, however, some exceptions. Floral bracts are caducous at anthesis in most of the Callerya group except in Adinobotrys atropurpureus, Nanhaia speciosa, Serawaia strobilifera and Wisteriopsis where they are persistent.

Gibbosities, which are small protuberances that develop beneath the leaf pulvinus above the stipule where it is attached to the stem, are absent in most of the Callerya group but are present in both Wisteriopsis, Nanhaia and Serawaia (Table 4; Fig. 2R-S). Bracteoles may be found either at the base of the calyx or along the pedicel. They are present in most genera but absent in Endosamara, Sigmoidala, Kanburia and Afgekia. They are also absent in Wisteria frutescens (Table 4).

Genera in the Callerya group often differ from each other (Table 4, Fig. 2) according to the presence of callosities at the base of the standard petals. Callosities occur in five distinct types:

a) Boss callosities form two slightly raised domes or swellings on either side of the midline of the standard lamina, at the point of its upward flexion above the claw (Fig. 2A). The standard in the latter case often appears to be smooth but the bosses hold the two wing petals close to the standard prior to anthesis. Boss callosities are found in Adinobotrys, Endosamara, Sigmoidala, Sarcodum, Nanhaia, Serawaia and Wisteriopsis and occasionally in Callerya and Whitfordiodendron;

b) Arched callosities are paired half-moon or crescent shaped arches forming ridges of hardened tissue that curve up from the base towards the midline over the staminal sheath (Fig. 2E). These are found only in Austrocallerya;

c) Ridge callosities form a straight ridge or rim of hardened tissue on either side of the midline of the standard near the base (Fig. 2B) and are seen in most Callerya s.str. species, Whitfordiodendron, Kanburia, Padbruggea dasyphylla and in the North American Wisteria frutescens;

d) Papillate callosities are those where a pair of papillate projections protrude from the area of hardened tissue on the surface usually at the point of upward flexion of the standard lamina above the claw (Fig. 2H). These are present in Afgekia, Padbruggea filipes (see Dunn 1911: 195 as Adinobotrys filipes) and all the Asian species of Wisteria (see Valder 1995: 26 as "auricles");

e) Corniculate callosities are present in the two species of Afgekia (Fig. 2I). Uniquely in the Callerya group, these two species have, in addition to a basal papillate pair, a second pair of corniculate or horned callosities which project out from the lamina above the basal pair. 

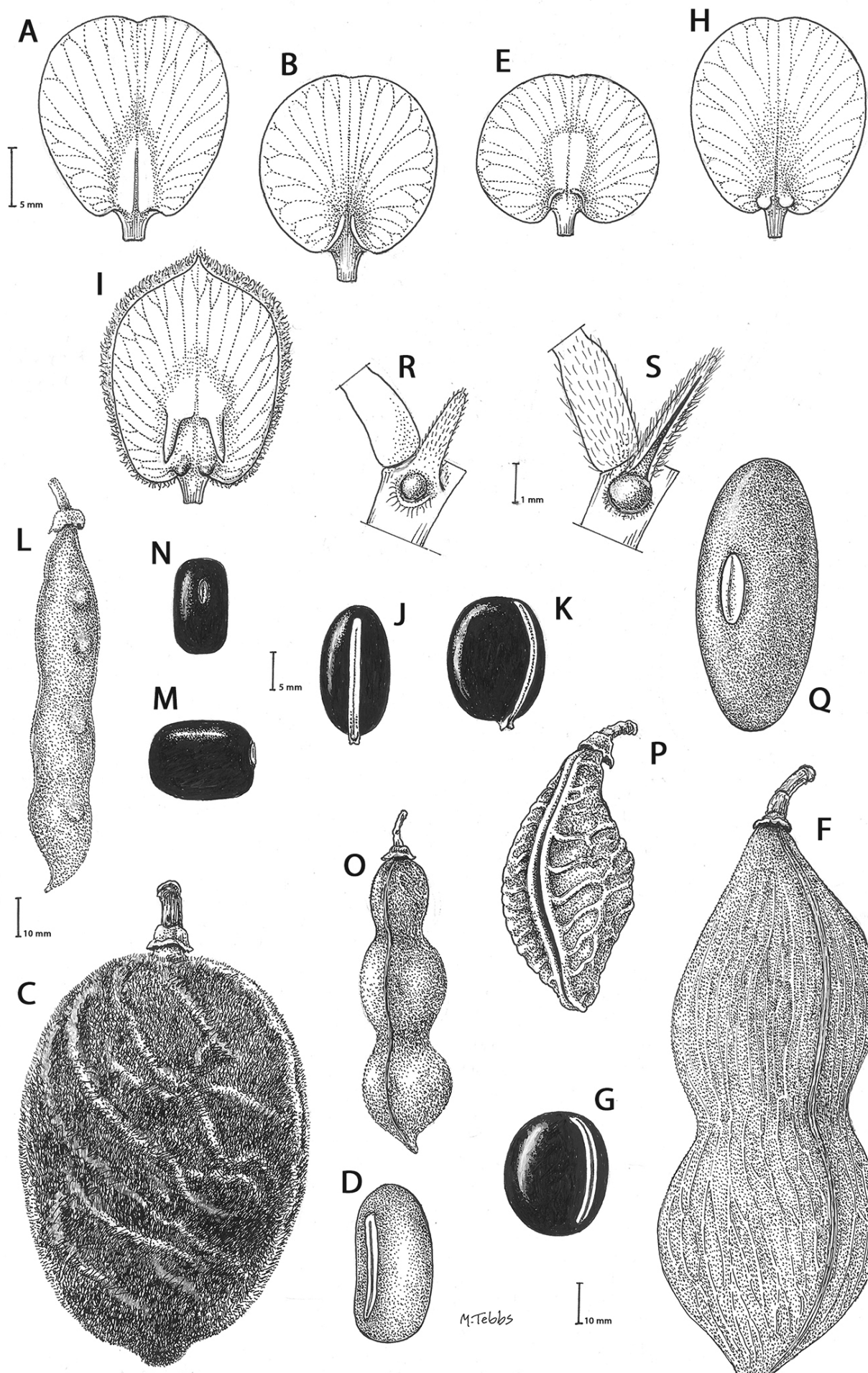
There are notable differences in the fruits and seeds among the genera. In Endosamara and Sarcodum the exocarp separates from the endocarp and some degree of separation also occurs in Wisteriopsis. In Endosamara the pods are clearly septate with transverse walls between each seed, forming loments (see Endosamara racemosa in Geesink 1984: 63 Pl. 1, 5; Lôc and Vidal 2001: 17, Pl. 3). In Sarcodum the sausageshaped or botuliform pods which initially have a fleshy exocarp, are also fully septate but do not form loments (see Sarcodum scandens in Geesink 1984: 63 Pl. 1, 7; Lôc and Vidal 2001: 9, Pl. 1). The North American Wisteria frutescens has nonseptate pods. In all other genera in the Callerya group the endocarp is subseptate with seeds making indentations in the surrounding pith while areas between the seeds appear as irregular, often indistinct transverse septa. In Afgekia the funicle as well as the hilum (see Afgekia sericea funicle in Geesink 1984: 64 Pl. 2, 10; Fig. 2J-K) are both significantly longer than those of other taxa in the group with the exception of Padbruggea (Fig. 2D) and Austrocallerya (Fig. 2G).

\section{Callerya group taxonomy I:The genus Callerya including genera previously placed in synonymy prior to this study}

\section{Callerya Endl., Gen. Pl. Suppl. 3: 104 (1843)}

Endlicher (1843) first described the genus Callerya based on Marquartia tomentosa Vogel, which had been described that same year from southern China (Vogel 1843: 37). Endlicher used the new name Callerya to replace Vogel's Marquartia, the replacement name being necessary because the generic name Marquartia Hassk. had already been provided a year earlier for a species of Pandanus by Justus Karl Hasskarl, assistant curator of the Buitenzorg Botanic Garden [Bogor] on Java (Hasskarl 1842: 14). Hasskarl's Marquartia is moreover, now considered as synonymous with Pandanus L.f. (1782).

In addition, the description and illustration of the species on which Vogel based his new but superfluous generic name Marquartia [i.e. M. tomentosa] (Vogel 1843) had

Figure 2. Distinctive morphological characters in Tribe Wisterieae. A Endosamara racemosa standard petal inner surface B Padbruggea dasyphylla standard petal inner surface C Padbruggea dasyphylla pod D Padbruggea dasyphylla seed lateral view $\mathbf{E}$ Austrocallerya australis standard petal inner surface $\mathbf{F}$ Austrocallerya pilipes pod G Austrocallerya pilipes seed lateral view $\mathbf{H}$ Padbruggea filipes standard petal I Afgekia sericea standard petal inner surface J Afgekia sericea seed lateral view K Afgekia sericea seed angled lateral view $\mathbf{L}$ Callerya nitida pod $\mathbf{M}$ Callerya nitida seed ventral view $\mathbf{N}$ Callerya nitida seed polar view $\mathbf{O}$ Callerya cinerea pod $\mathbf{P}$ Whitfordiodendron nieuwenhuisii pod $\mathbf{Q}$ Whitfordiodendron erianthum seed $\mathbf{R}$ Wisteriopsis eurybotrya gibbosity $\mathbf{S}$ Wisteriopsis championii gibbosity A from Luang Vanpruk 188 B from Scortechini 429 C, D from Lamb 395/91 E from L.J.Brass 32129 F, G from B. Gray 04319 H from Maung Po Khant 15326 I from C. Chermsirivathana $996 \mathbf{J ,} \mathbf{K}$ from Mrs Collins 104/9 L-N from Theophilus Sampson $\mathbf{O}$ from G.Forrest $19279 \mathbf{P}$ from J.P.Mogea $4182 \mathbf{Q}$ from photo Y.Sirichamorn s.n. $\mathbf{R}$ from J. \& M.S.Clemens 3637 $\mathbf{S}$ from Shiu Ying Hu 10476. See Appendix 1 for voucher details. Drawn by Margaret Tebbs. 


\begin{tabular}{|c|c|c|c|c|c|c|c|c|c|c|c|}
\hline 苞 & 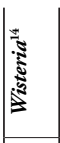 & 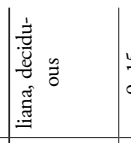 & $\frac{n}{d}$ & 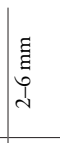 & 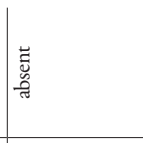 & 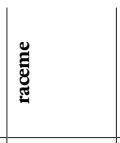 & 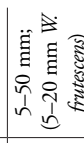 & 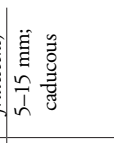 & 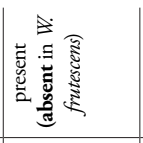 & 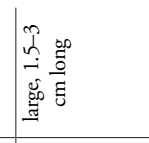 & 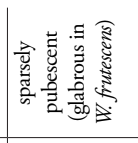 \\
\hline & รี่ & 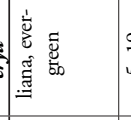 & ने & $\begin{array}{l}\text { E } \\
\text { E } \\
0 \\
1 \\
\\
-\end{array}$ & 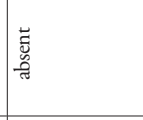 & 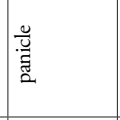 & 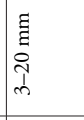 & 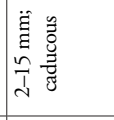 & 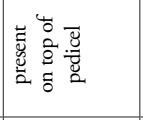 & 焉 & 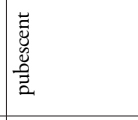 \\
\hline 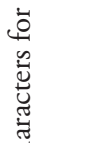 & 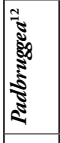 & 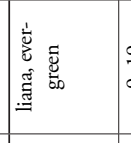 & $\frac{\partial}{\partial}$ & $\begin{array}{l}\text { E } \\
\text { g } \\
\text { D } \\
-1\end{array}$ & 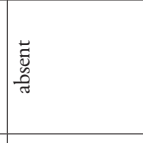 & 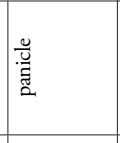 & 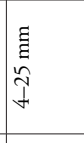 & 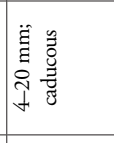 & 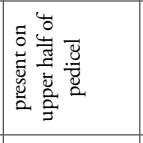 & 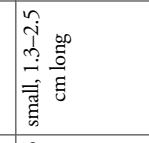 & 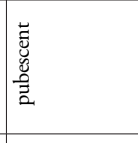 \\
\hline 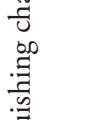 & 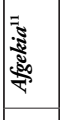 & 宽 & $\bar{\partial}$ & 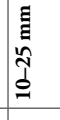 & 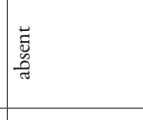 & 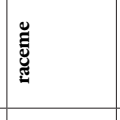 & 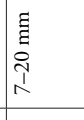 & 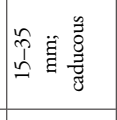 & 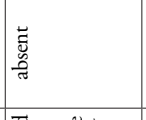 & 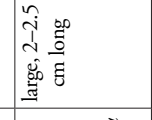 & 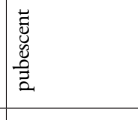 \\
\hline 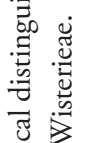 & ป & 这 & $\frac{m}{m}$ & 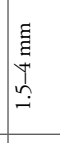 & | & 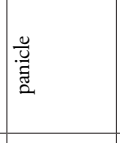 & 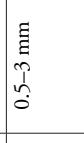 & 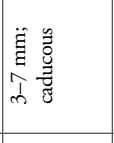 & 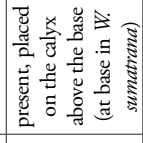 & 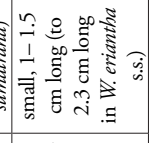 & 咢 \\
\hline 苞 & है & 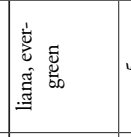 & in & 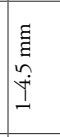 & | & 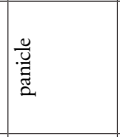 & 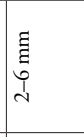 & 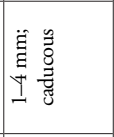 & 蒿 & 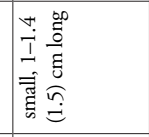 & 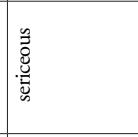 \\
\hline 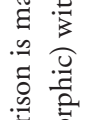 & 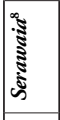 & 㞼 & in & $\begin{array}{l}E \\
\vdots \\
\infty \\
0 \\
\sim\end{array}$ & 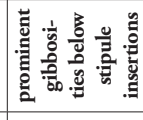 & 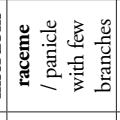 & E & 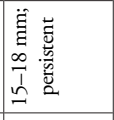 & 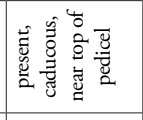 & 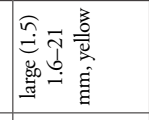 & 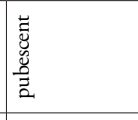 \\
\hline 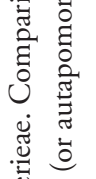 & ड़ี & 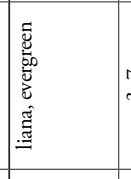 & î & 吾 & 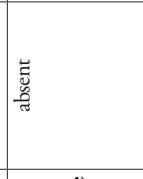 & 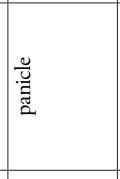 & $\begin{array}{l}E \\
\vdots \\
\infty \\
d \\
\text { c }\end{array}$ & 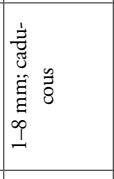 & 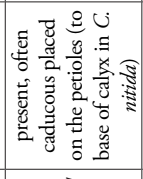 & 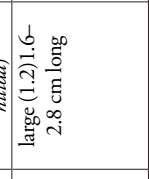 & 号 \\
\hline 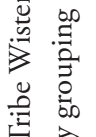 & 気 & 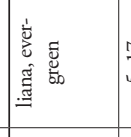 & in & E & 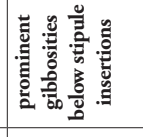 & 总 & 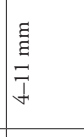 & 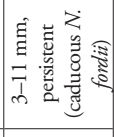 & 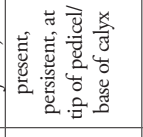 & 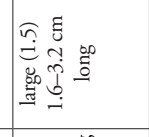 & 产 \\
\hline 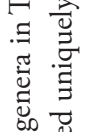 & 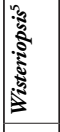 & 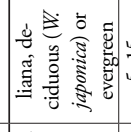 & 9 & $\underset{\Xi}{E}$ & 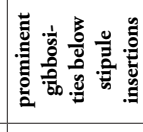 & 产 & $\begin{array}{l}E \\
\vdots \\
\text { I }\end{array}$ & 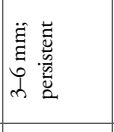 & 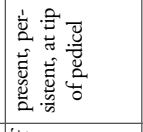 & 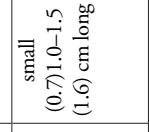 & 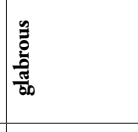 \\
\hline 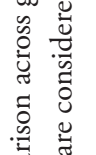 & है & 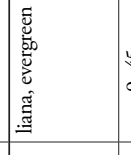 & a & 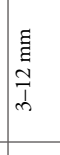 & 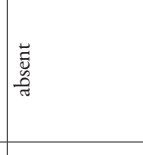 & 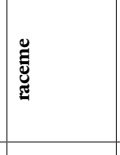 & 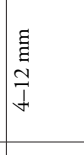 & 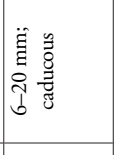 & 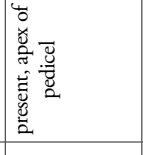 & 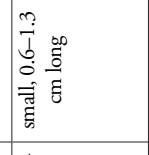 & 产 \\
\hline 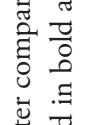 & 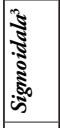 & 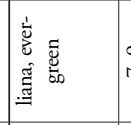 & $i$ & $\begin{array}{l}\text { E } \\
\text { g } \\
0 \\
\text { m }\end{array}$ & 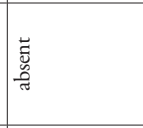 & . & $\begin{array}{l}E \\
E \\
m \\
m\end{array}$ & 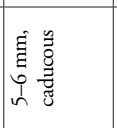 & 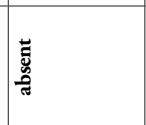 & 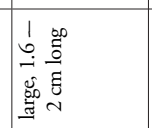 & 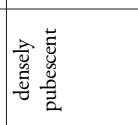 \\
\hline 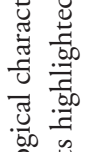 & है & 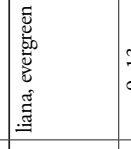 & $\frac{m}{d}$ & $\begin{array}{l}E \\
\vdots \\
0 \\
\vdots \\
n \\
n\end{array}$ & 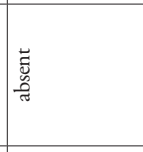 & 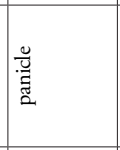 & $\begin{array}{l}E \\
\Xi \\
g \\
0 \\
m\end{array}$ & 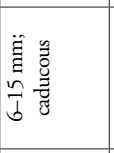 & 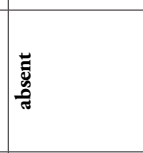 & 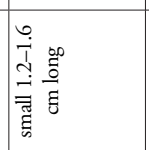 & 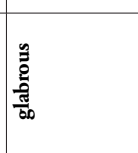 \\
\hline 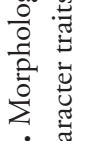 & 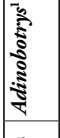 & 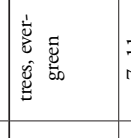 & $\overline{1}$ & $\underset{\Xi}{E}$ & 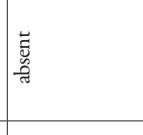 & . & $\begin{array}{l}\text { E } \\
\vdots \\
0 \\
\vdots \\
n\end{array}$ & 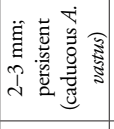 & 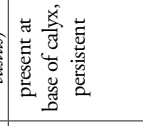 & 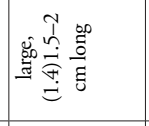 & 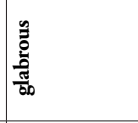 \\
\hline 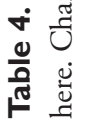 & 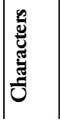 & 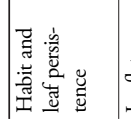 & 芭 & & 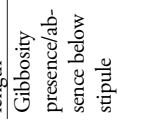 & 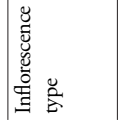 & 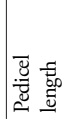 & 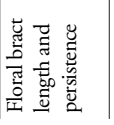 & 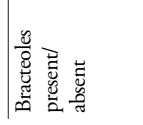 & 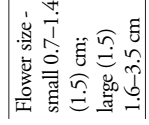 & 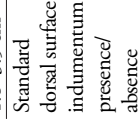 \\
\hline
\end{tabular}




\begin{tabular}{|c|c|c|c|c|c|c|c|}
\hline$\frac{\sqrt{2}}{2 \pi}$ & 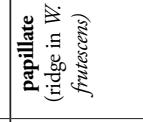 & 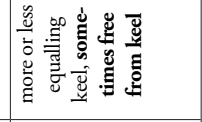 & 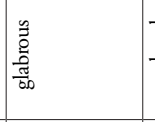 & 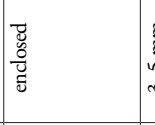 & $\begin{array}{c}E \\
\vdots \\
\omega \\
\omega\end{array}$ & 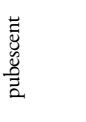 & 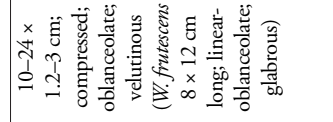 \\
\hline & $\vec{z}$ & 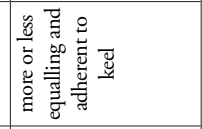 & 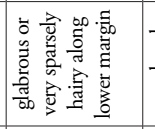 & 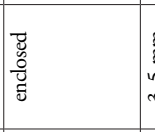 & $\begin{array}{c}\xi \\
\xi \\
\sim \\
n \\
m\end{array}$ & 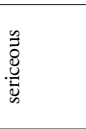 & 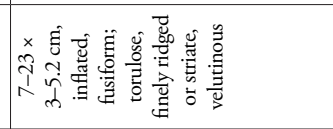 \\
\hline 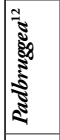 & 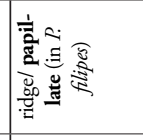 & 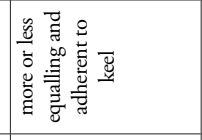 & 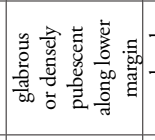 & $\frac{\bar{u}}{\tilde{u}}$ & $\begin{array}{l}E \\
\vdots \\
⿱ 亠 䒑 \\
m\end{array}$ & : & 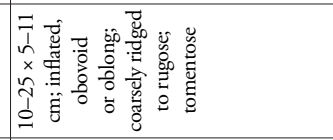 \\
\hline 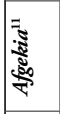 & 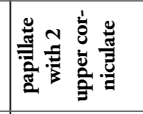 & 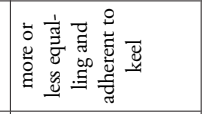 & 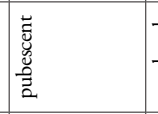 & \begin{tabular}{|l} 
\\
$\bar{g}$ \\
$\frac{g}{\bar{g}}$ \\
$\bar{\Xi}$
\end{tabular} & $\underset{\Xi}{\Xi}$ & 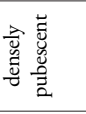 & 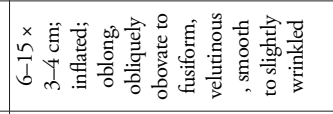 \\
\hline 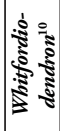 & 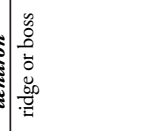 & 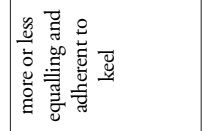 & 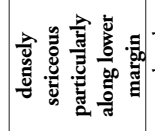 & & 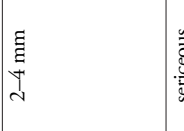 & 萢 & 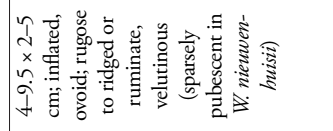 \\
\hline है & م: & 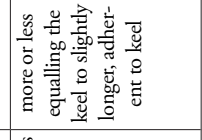 & $\frac{y}{2}$ & 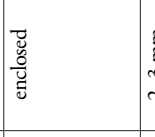 & 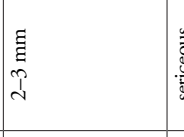 & 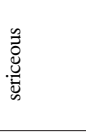 & 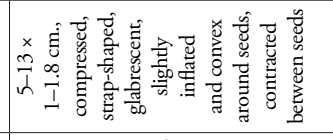 \\
\hline 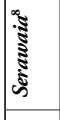 & 总 & 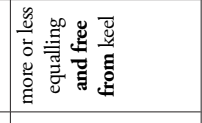 & 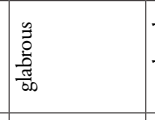 & 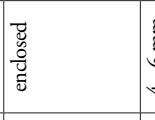 & $\begin{array}{l}\text { घ. } \\
\text { घु } \\
\vdots \\
\vdots \\
\text { W }\end{array}$ & 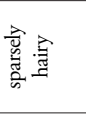 & 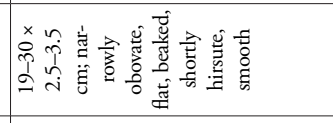 \\
\hline בิ & 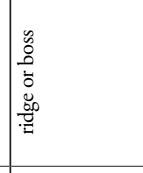 & 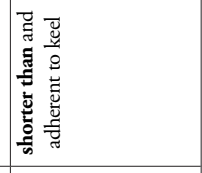 & $\frac{y}{0}$ & $\begin{array}{l}\bar{\Xi} \\
\overline{\mathscr{J}} \\
\bar{\Xi} \\
\bar{\Xi}\end{array}$ & 音 & & 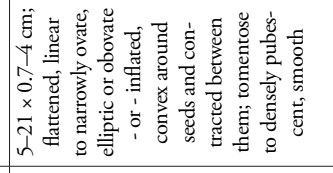 \\
\hline है & 参 & 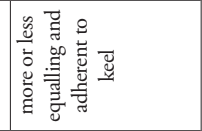 & 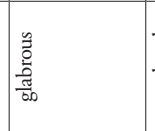 & 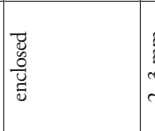 & $\begin{array}{l}\text { घ } \\
\vdots \\
\sim \\
\sim \\
\sim\end{array}$ & 蒫 & 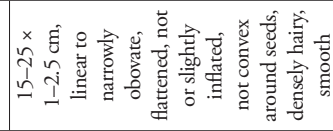 \\
\hline 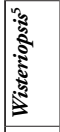 & 叁 & 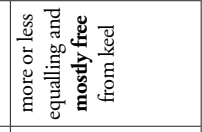 & 产 & Ё & 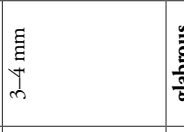 & 总 & 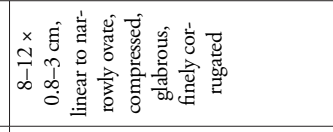 \\
\hline 赔 & 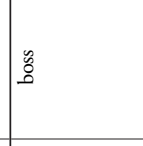 & 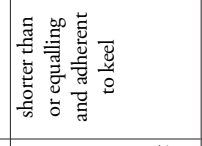 & 产 & 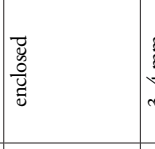 & $\begin{array}{l}E \\
g \\
\dot{m} \\
\text { m }\end{array}$ & 产 & 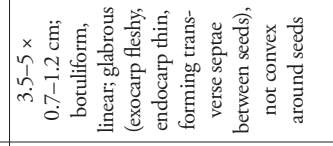 \\
\hline 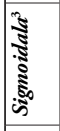 & 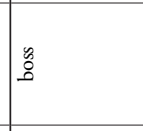 & 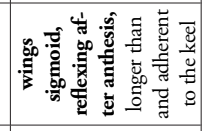 & 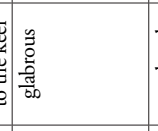 & 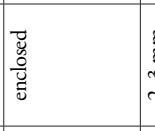 & $\begin{array}{l}E \\
\text { g } \\
\stackrel{d}{d}\end{array}$ & 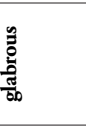 & 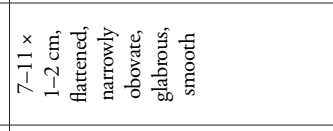 \\
\hline है & 岁 & 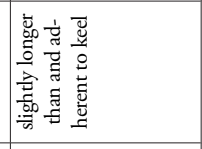 & 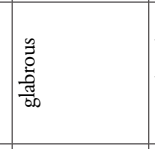 & 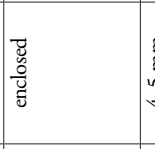 & 音 & 产 & 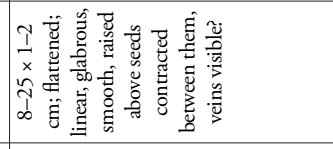 \\
\hline 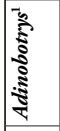 & 岁 & 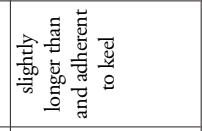 & 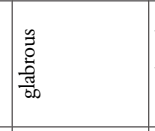 & 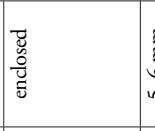 & 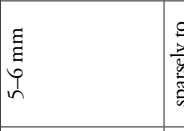 & 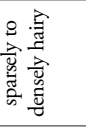 & 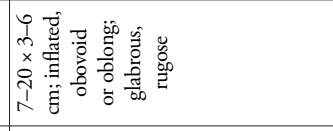 \\
\hline 䓀 & 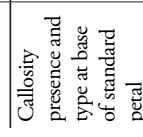 & 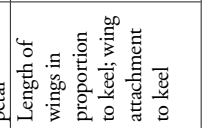 & 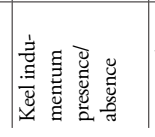 & 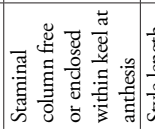 & 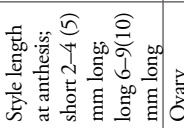 & 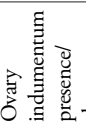 & 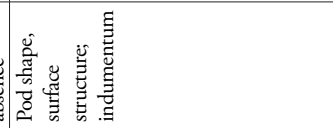 \\
\hline
\end{tabular}




\begin{tabular}{|c|c|c|c|c|}
\hline 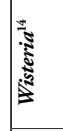 & 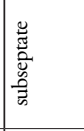 & 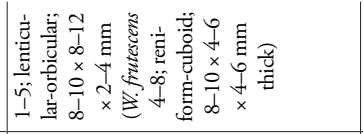 & 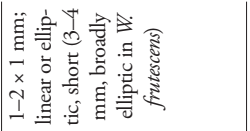 & 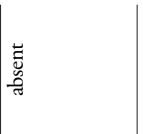 \\
\hline है है & 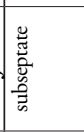 & 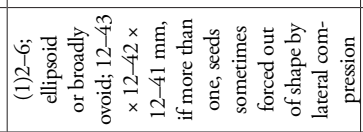 & 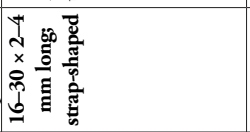 & 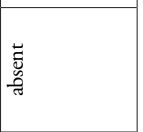 \\
\hline 赔 & 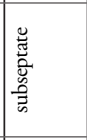 & 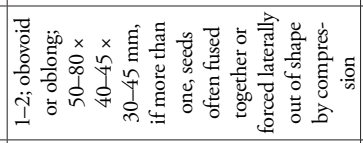 & 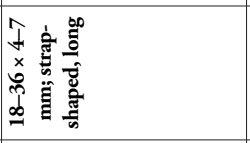 & 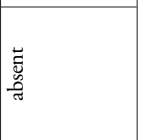 \\
\hline ॠ & 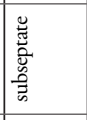 & 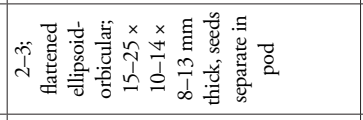 & 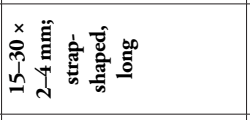 & 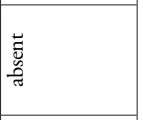 \\
\hline : & 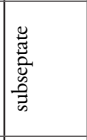 & 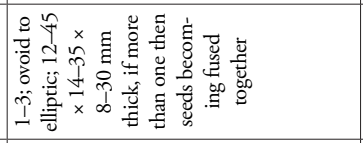 & 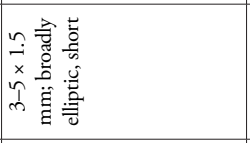 & 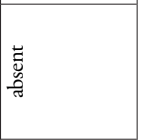 \\
\hline & 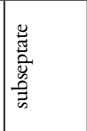 & 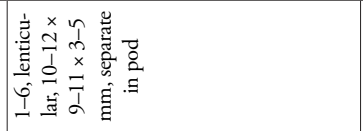 & 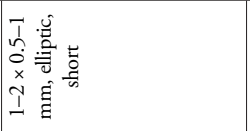 & 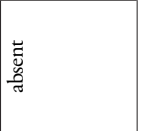 \\
\hline & 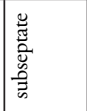 & 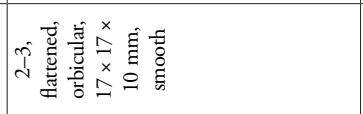 & 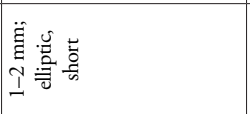 & 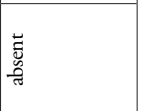 \\
\hline & 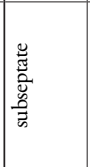 & 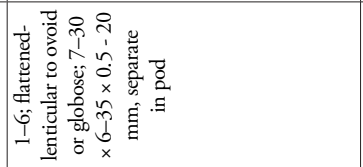 & 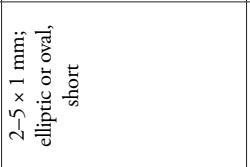 & 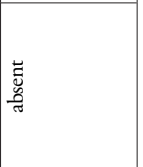 \\
\hline & 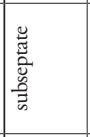 & 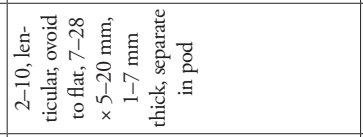 & 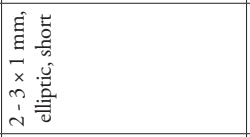 & 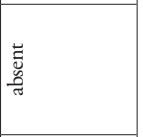 \\
\hline 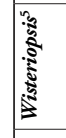 & 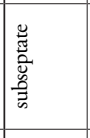 & 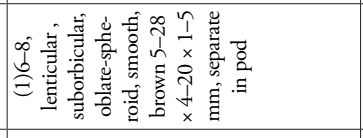 & 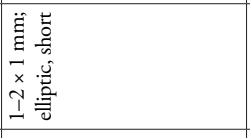 & 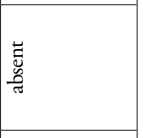 \\
\hline & 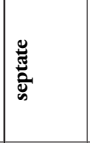 & 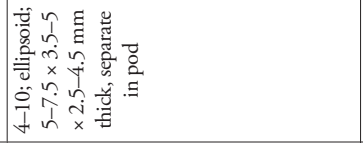 & 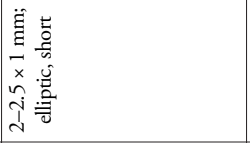 & 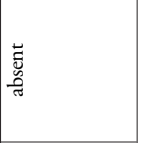 \\
\hline & 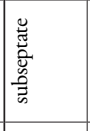 & 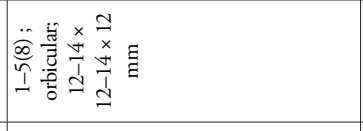 & 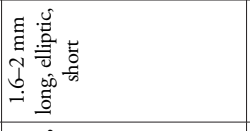 & 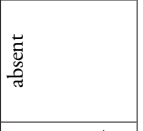 \\
\hline है & 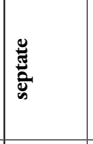 & 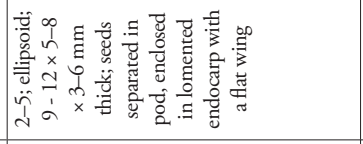 & 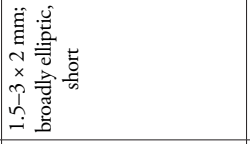 & 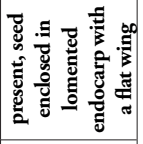 \\
\hline हैं & 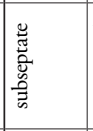 & 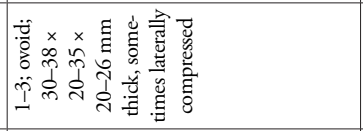 & 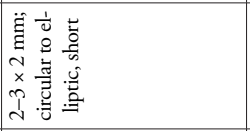 & 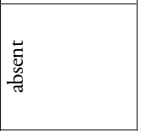 \\
\hline Uू. & 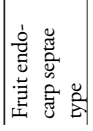 & 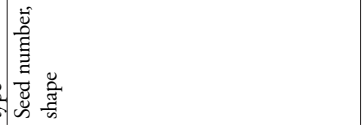 & 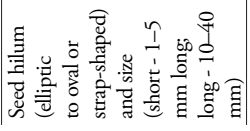 & \\
\hline
\end{tabular}


already been described by Bentham as Millettia nitida Benth. (Bentham 1842: 484). Vogel's illustration clearly shows the leaves with persistent stipules, each with five leaflets, long persistent floral bracts and densely sericeous ovary, all characters diagnostic of that species. This meant that not only the generic name Marquartia but also Callerya tomentosa (Vogel) Endl. had to be replaced according to Art. 11.4 of the ICN (Turland et al. 2018). The replacement Callerya nitida (Benth.) Geesink was published formally by Geesink within his revision of Tribe Millettieae (Geesink 1984: 82). Callerya nitida is thus the type species of the genus Callerya. Endlicher's description of Callerya mentioned the compressed, woody and leathery pod which he stated contained either a few seeds or a single seed which was ovoid-circular and flattened-compressed. Callerya nitida (Fig. 2L-N) does indeed possess flattened pods with up to ten compressed seeds (see Schot 1994: 28, Fig. 3). The species may also become a scandent shrub but is not arborescent as Endlicher implied.

Geesink (1984) recognised that species of Callerya had true paniculate inflorescences. He also sank three genera; Adinobotrys Dunn, Padbruggea Miq. and Whitfordiodendron Elmer into his redefined Callerya and transferred two Sections created by Dunn (1912a) from Millettia - Sect. Eurybotryae Dunn and Sect. Austromillettia Dunn - into Callerya and created a new monotypic genus Endosamara Geesink from Sect. Bracteatae Dunn based on Robinia racemosa Roxb. (1832).

Callerya was revised by Schot (1994) and was treated as belonging in Tribe Millettieae (see Table 1). Schot recognised 19 species from China, south-east Asia and Australasia and since then many more species have been described, bringing the total number to 33 (Sirichamorn et al. 2016). All species sensu Schot (1994) except C. atropurpurea and $C$. vasta, are vigorous climbing or scandent woody shrubs. Inflorescences are paniculate with either axillary racemes or secondary panicles and frequently possess rather thick to woody inflorescence axes with prominent bud scars. Floral bracts are generally short and can be narrow or broad according to species and are in most species caducous, rarely persistent. Flowers may be white, green, red, brownish-yellow, lilac, pink or deep purple. In the type species Callerya nitida, the wings are distinctly shorter than the keel. Fruits, which can be either flattened or inflated are velutinous and ribbed, occasionally wrinkled or smooth and the seed chambers are subseptate. Seeds 2-9, large, ovoid to ellipsoid (Tables 1, 4).

Schot (1994: 2) chronicled the transfer of species from other genera into Callerya and included eleven species from Millettia, the first two of which were Pterocarpus australis Endl. Prod. Fl. Norfolk (Endlicher 1833: 49) and Pongamia atropurpurea Wall. (Wallich 1830: 70).

Although the genus Pterocarpus is placed in Tribe Dalbergieae (Klitgaard and Lavin 2005), the species $P$. australis has been shown to belong in the Callerya group (Li et al. 2014). Pongamia Adans. is now treated as being synonymous with Millettia, with the type $P$. pinnata (L.) Pierre being transferred to Millettia pinnata (L.) Panigrahi by Panigrahi and Murti (1989). Pongamia atropurpurea which was transferred into Callerya by Schot (1994: 15) has also been found to belong in the Callerya group (Li et al. 2014). Schot (1994) further transferred the Australian species originally described 
as Wisteria megasperma F.Muell. (1858) into Callerya. This too has been confirmed to belong within the Callerya group (Li et al. 2014).

In their analyses using combined data from chloroplast $\operatorname{trn} K$ and $m a t K$ sequences, Hu et al. (2000) showed that Callerya reticulata was sister to a clade supported by BS $100 \%$ with Wisteria frutescens and W. sinensis. In a later paper using sequence data from nuclear DNA ITS spacers and a larger sampling of Callerya and Wisteria as well as Afgekia filipes, Hu et al. (2002) found that Callerya was polyphyletic occurring in four different clades. Wisteria frutescens was strongly supported sister to W. brachybotrys, W. sinensis and W. floribunda with BS 100\%. The Wisteria clade was sister to a clade with Callerya megasperma, C. australis and Afgekia filipes with strong BS (85\%) support. In a later analysis $\mathrm{Hu}$ and Chang (2003) using the more conserved $r b c L$ chloroplast gene, found that Callerya vasta was early branching to a clade of Wisteria sinensis sister to Afgekia sericea while Endosamara racemosa was sister to Millettia japonica.

Schot included nine synonyms within her concept of $C$. cinerea, a species that she recognised to have a wide distribution from Nepal in the west to the Chinese coast in the east (Schot 1994: 17). In our analyses we have utilised two specimens of Callerya cinerea, one from Thailand, the other from China, (Table 2) but these sheets lack diagnostically significant fruiting material and may not equate fully with the holotype material seen at Kew from Bangladesh, Sylhet, Chittagong, (Wallich 5888; K000881022). Within the C. cinerea complex, leaflet number, pod thickness and seed shape and number appear to be important characters. Wei and Pedley (2010) split $C$. cinerea into groups of species based on leaflet number: $3-5$ in $C$. tsui, C. dorwardii and C. sphaerosperma and 5-7 in the other seven species. Wei and Pedley (2010) also segregated the species on the degree of pod inflation: flattened with lenticular seeds in $C$. congestiflora, $C$. dielsiana and C. longipedunculata; inflated with globose seeds in $C$. cinerea, $C$. dorwardii, C. gentiliana, C. oosperma and $C$. sericosema. We have only been able to sample material of two of the resurrected species recognised by Wei and Pedley (2010) that were included in $C$. cinerea by Schot (1994), namely C. dielsiana and C. oosperma. A further investigation of this group of Chinese species is needed to fully assess species delimitations. If all these species belong together with $C$. nitida and $C$. cochinchinensis as indicated both by $\mathrm{Li}$ et al. (2014) and from our preliminary results here, it seems that Callerya s.str. might comprise as many as twelve species.

\section{Padbruggea Miq. Fl. Ned. Ind. 1(1): 150 (1855)}

Miquel (1855: 150) described the genus Padbruggea including the statement:

"legumen oblongum, stipitatum crassum? exalatum" [legume oblong, possibly on a thickened stipe and not winged] and he particularly noted the presence of callosities on the standard petal "vexillum infra medium quidem texturae crassioris ac perinde subfoveolatum" [standard with a thickened area just below the middle with a somewhat pitted texture] 
Reference to the unwinged nature of the fruit was in comparison to some species of Pterocarpus Jacq. (Tribe Dalbergieae) whose fruits have a distinct wing-like exocarp. Miquel also noted that he had not seen mature fruits.

Our examination of the species described as $P$. dasyphylla Miq. (1855), revealed that the pods were readily distinguished from others in the Callerya group by their inflated but broadly flattened-cuboid shape with distinct longitudinal ridges and furrows and by the 1 or 2 compressed obovoid seeds possessing long strap-shaped hila 18-36 $\times$ 4-7 mm (Table 5).

In his protologue of Adinobotrys filipes, Dunn (1911: 196) noted that in its appearance the species had more slender pedicels than those in what he considered to be the closely related A. erianthus (here in Whitfordiodendron) and that it approached Padbruggea in its auriculate standard (Dunn 1911). Dunn's latter reference may also refer to the papillate callosities present on the standard of $A$. filipes but which are absent on the smooth standard of A. erianthus. In her monograph on Callerya, Schot (1994: 3) commented:

Craib (1928) argued that Padbruggea and Whitfordiodendron were congeneric. He based his arguments on the intermediate position of Adinobotrys filipes Dunn (now Afgekia filipes Geesink). This species resembles in habit mostly Padbruggea dasyphylla, but has the generic characters of Adinobotrys.

Craib recombined Adinobotrys filipes in Padbruggea, along with a good measure of uncertainty as to whether he believed the species really belonged in that genus or in Adinobotrys (Craib 1928: 397). He also postulated that Elmer's Whitfordiodendron may belong in Padbruggea thereby highlighting the morphological difficulties with respect to these taxa faced by later workers such as Geesink (1984).

Schot in her synonymy of Callerya dasyphylla also included Milletia oocarpa Prain, distinguished from $P$. dasyphylla by its ovoid as opposed to compressed obovoid fruits and M. maingayi Baker which differs in its more numerous, smaller and more densely tomentose leaflets (Schot 1994: 20). We have recognised this as Padbruggea maingayi in this paper (see below).

The status of Afgekia filipes has long been debated as it has true panicles as opposed to racemes and shorter calyx teeth than those of the other two species of Afgekia (see Table 5). It also has a single pair of papillate callosities on the standard as opposed to two pairs found in both A. sericea and A. mabidoliae. Afgekia filipes has entirely glabrous anthers as opposed to anthers with a basal tuft of hairs and it has much larger fruits and seeds (Table 5). It was originally described as Adinobotrys filipes Dunn on the basis of its large single seeded pods (Dunn 1911: 195).

Geesink (1984: 76) transferred Adinobotrys filipes into Afgekia adding:

the general habit, the shape of the calyx, and the glabrous anthers are indeed similar to certain species of Padbruggea. It differs in the absence of bracteoles and the long pedicels. The pods were unknown until 1975, but then it appeared that the seeds showed an elongated fleshy funicle with a corresponding elongated hilum. 
Geesink (1984: 76) concluded that the morphology of $A$. filipes indicated that it was a less derived species than $A$. sericea and $A$. mahidoliae and alluded to its affinities with Padbruggea within which it had been placed by Craib (1928) along with three other species in Adinobotrys.

In his transferral of the species into Afgekia, Geesink noted the apparent absence of bracteoles, the length of the pedicels and the elongated hilum on the seeds (Geesink 1984: 77). Both Lôc and Vidal (2001) and Wei and Pedley (2010) followed Geesink, maintaining the species in Afgekia. Sirichamorn (2006) examined 37 living specimens of $A$. sericea, 50 specimens of $A$. mahidoliae and 32 specimens of $A$. filipes from wild material in Thailand and found that $A$. filipes posseses bracteoles, that pedicel length among the species overlaps and that the overall size of the seeds are three times that of the other two species of Afgekia, i.e. c. $80 \mathrm{~mm}$ vs. 15-25 mm long (Tables 4, 5).

Prathepha and Baimai (2003) mentioned the existence of Afgekia filipes in their RAPD and nucleotide sequence analyses of $A$. mahidoliae and $A$. sericea, although they did not state their reason for excluding the species. Sirichamorn (2006), based on morphometric and molecular data, clearly showed that Adinobotrys filipes does not belong with Afgekia (Table 5).

We have examined material of both Afgekia filipes and Callerya dasyphylla and agree that there are indeed similarities between the two species. We have confirmed Sirichamorn's (2006) discovery that Afgekia filipes does have short, linear bracteoles that are attached at the base of the calyx (Table 5). Both species possess inflated fruits with a velvety indumentum and oblique longitudinal ridges and furrows but those of $C$. dasyphylla are broader and flatter, with the dorsal midline flanked by two large folds or flanges that meet at the apex. Our results show that Afgekia filipes, originally described by Dunn (1911) in Adinobotrys, belongs in the genus Padbruggea and it is reinstated in that genus here (see Taxonomic treatment below) following Craib (1928). The diagnostic characters of Padbruggea filipes are shown in Table 5.

\section{Whitfordiodendron Elmer. Leafl. Philipp. Bot. 2: 689, 743 (1910)}

Elmer (1910) in his protologue of the illegitimate but valid name Whitfordia scandens stated that he had only seen young not mature fruits but that they were "thick, hard, canescently velvety and 1-seeded". He also noted the puberulent dorsal surface of the standard petal of the deep red flowers (Elmer 1910: 691). The generic name Whitfordia was already utilised for the fungal genus Whitfordia Murrill (Murrill 1908: 407), a synonym of Amauroderma Murrill (Murrill 1905: 366) and although Whitfordia Elmer (with W. scandens) was described entirely in English, together with that of the transfer to Whitfordiodendron as an erratum in an appendix to the same volume, the name is nevertheless still valid. Under the International Rules of Nomenclature adopted in Vienna in 1905 and Brussels in 1910, a Latin diagnosis was a requirement for valid publication of a name of a new taxon on or after 1 January 1908 (Art. 36). As a result of discrepancies and disagreements between the American Code of Botanical Nomen- 
Table 5. Morphological characters distinguishing Afgekia and Padbruggea. Comparison is made of critical characters of these genera in support of the transfer of A. filipes to Padbruggea.

\begin{tabular}{|c|c|c|c|c|}
\hline List of characters & $\begin{array}{l}\text { Padbruggea } \\
\text { dasyphylla }\end{array}$ & $\begin{array}{c}\text { Padbruggea } \\
\text { (=Afgekia) filipes }\end{array}$ & Afgekia sericea & Afgekia mabidoliae \\
\hline Stem sap colour & blood red & blood red & colourless & colourless \\
\hline Stipule type & $\begin{array}{c}4-8 \mathrm{~mm} \text {, ovate- } \\
\text { lanceolate, caducous }\end{array}$ & $\begin{array}{l}\text { 1-2.5 mm; deltoid; } \\
\text { caducous }\end{array}$ & $\begin{array}{c}15-25 \mathrm{~mm} ; \\
\text { ovate-lanceolate; } \\
\text { persistent }\end{array}$ & $\begin{array}{l}\text { 10-20 mm; ovate- } \\
\text { lanceolate; persistent }\end{array}$ \\
\hline Leaflet number & $9-17$ & $13-19$ & $15-17$ & $9-11$ \\
\hline Inflorescence type & $\begin{array}{l}\text { multi-branched } \\
\text { panicle }\end{array}$ & multi-branched panicle & $\begin{array}{l}\text { simple axillary } \\
\text { raceme }\end{array}$ & simple axillary raceme \\
\hline Inflorescence axis & $\begin{array}{l}\text { robust, thickened, } \\
\text { woody, }\end{array}$ & $\begin{array}{l}\text { robust, often thickened } \\
\text { and becoming woody }\end{array}$ & $\begin{array}{l}\text { slender, not } \\
\text { thickened or } \\
\text { woody }\end{array}$ & $\begin{array}{l}\text { slender, not thickened or } \\
\text { woody }\end{array}$ \\
\hline Pedicel length & 4-7 $\mathrm{mm}$ long & $15-25 \mathrm{~mm}$ long & $7-20 \mathrm{~mm}$ long & $7-10 \mathrm{~mm}$ long \\
\hline Calyx teeth & acute; $1-3 \mathrm{~mm}$ long & obtuse; 3-6 $\mathrm{mm}$ long & $\begin{array}{l}\text { linear-lanceolate; } \\
\text { 4-17 mm long }\end{array}$ & $\begin{array}{l}\text { linear-lanceolate; 5-15 } \\
\text { mm long }\end{array}$ \\
\hline Floral bracteole & $\begin{array}{l}\text { 3-6 mm long; } \\
\text { caducous }\end{array}$ & $\begin{array}{l}0.5-1 \text { mm long; } \\
\text { caducous }\end{array}$ & absent & absent \\
\hline Floral bract type & $\begin{array}{l}\text { 4-5 mm long, ovate; } \\
\text { apex acute; as wide as } \\
\text { flower buds at anthesis }\end{array}$ & $\begin{array}{l}\text { 15-20 mm long; } \\
\text { broadly ovate; apex } \\
\text { acute; wider than } \\
\text { flower buds prior to } \\
\text { anthesis }\end{array}$ & $\begin{array}{c}\text { 20-35 mm } \\
\text { long; lanceolate; } \\
\text { apex attenuate; } \\
\text { narrower than } \\
\text { flower buds at } \\
\text { anthesis }\end{array}$ & $\begin{array}{l}\text { 15-30 mm long; } \\
\text { lanceolate; apex attenuate; } \\
\text { narrower than flower buds } \\
\text { at anthesis }\end{array}$ \\
\hline Floral fragrance & fragrant & fragrant & scentless & scentless \\
\hline $\begin{array}{l}\text { Callosities on } \\
\text { standard petal }\end{array}$ & 1 pair; ridge type & 1 pair; papillate & $\begin{array}{l}2 \text { pairs; } 1 \\
\text { papillate, } 1 \\
\text { corniculate }\end{array}$ & $\begin{array}{l}2 \text { pairs; } 1 \text { papillate, } 1 \\
\text { corniculate }\end{array}$ \\
\hline $\begin{array}{l}\text { Wing petal } \\
\text { appendage at base }\end{array}$ & one claw & one claw & two claws & one claw \\
\hline $\begin{array}{l}\text { Keel petal shape } \\
\text { and pubescence }\end{array}$ & $\begin{array}{l}\text { white or pale pink; } \\
\text { glabrous; falcate }\end{array}$ & $\begin{array}{c}\text { white or lilac; glabrous; } \\
\text { cochleate }\end{array}$ & $\begin{array}{l}\text { white; densely } \\
\text { pubescent; } \\
\text { naviculate }\end{array}$ & $\begin{array}{c}\text { white; densely pubescent; } \\
\text { naviculate }\end{array}$ \\
\hline $\begin{array}{l}\text { Filament hairs } \\
\text { above and below } \\
\text { anthers }\end{array}$ & absent & absent & present & present \\
\hline $\begin{array}{l}\text { Pod size, shape } \\
\text { and surface } \\
\text { ornamentation }\end{array}$ & $\begin{array}{c}100-170 \text { x } 50-90 \\
\text { mm; oblong; apex } \\
\text { obtuse; velutinous; } \\
\text { obliquely ridged }\end{array}$ & $\begin{array}{c}170-250 \times 50-110 \\
\text { mm; ovoid-fusiform; } \\
\quad \text { apex obtuse; } \\
\text { velutinous; obliquely } \\
\text { ridged and furrowed }\end{array}$ & $\begin{array}{c}70-150 \times 30-40 \\
\text { mm; ellipsoid- } \\
\text { obovoid; apex } \\
\text { acute; velutinous; } \\
\text { smooth, lacking } \\
\text { ridges }\end{array}$ & $\begin{array}{c}\text { 60-90 × 30-35 mm; } \\
\text { ellipsoid-obovoid; apex } \\
\text { acute; velutinous; smooth, } \\
\text { lacking ridges }\end{array}$ \\
\hline $\begin{array}{l}\text { Seed size, number } \\
\text { per fruit, shape } \\
\text { and surface texture }\end{array}$ & $\begin{array}{c}40-50 \times 30-40 \mathrm{~mm} ; \\
1 \text { or } 2 \text {; oblong, testa } \\
\text { smooth }\end{array}$ & $\begin{array}{c}60-80 \times 40-50 \mathrm{~mm} ; \\
1, \text { rarely } 2 ; \text { oblong- } \\
\text { orbicular, testa rugose } \\
\text { or wrinkled }\end{array}$ & $\begin{array}{c}15-20 \times \\
10-12 \mathrm{~mm} ; 2 \\
\text { or 3; lenticular- } \\
\text { orbicular, testa } \\
\text { smooth, glossy }\end{array}$ & $\begin{array}{c}18-25 \times 12-14 \mathrm{~mm} ; 2 \text { or } \\
3 \text {; lenticular-orbicular, testa } \\
\text { smooth, glossy }\end{array}$ \\
\hline $\begin{array}{l}\text { Hilum shape and } \\
\text { length }\end{array}$ & $\begin{array}{c}18-20 \times 4-5 \mathrm{~mm} ; \\
\text { narrowly elliptic; } 1 / 3 \\
\text { circumference of seed }\end{array}$ & $\begin{array}{l}20-36 \times 5-7 \mathrm{~mm} ; \\
\text { narrowly elliptic; } 1 / 6 \\
\text { to } 1 / 8 \text { circumference } \\
\text { of seed }\end{array}$ & $\begin{array}{c}15-22 \times 2-4 \\
\text { mm; ligulate; } 1 / 2 \\
\text { circumference of } \\
\text { seed }\end{array}$ & $\begin{array}{l}18-30 \times 2-4 \mathrm{~mm} \text {; ligulate; } \\
1 / 2 \text { circumference of seed }\end{array}$ \\
\hline
\end{tabular}


clature [see Bull. Torrey Bot. Club 34(4): 167-178 (1907)] and the Vienna Rules and Brussels Rules, a rapprochement was made in the Cambridge Rules (1935) changing the implementation date to 1935. As a result, Elmer's Whitfordiodendron based on W. scandens Elmer is validly published (J. McNeill pers. comm.).

Despite the nomenclatural wrangles on the validity and usage of Adinobotrys versus Whitfordiodendron (discussed by Merrill [1934: 159] who independently concluded that Art. 38 of the Cambridge Rules validated Elmer's Whitfordiodendron), Merrill proceeded to describe the new species Whitfordiodendron sumatranum Merr., which he stated was close to W. myrianthus (i.e. to W. nieuwenhuisii (J.J.Sm.) Dunn). Merrill (1934: 160) also made what he believed to have been four new combinations in Whitfordiodendron but he was evidently unaware that W. atropurpureum, W. erianthum, W. myrianthum and W. nieuwenhuisii had already been combined in Whitfordiodendron by Dunn (1912b: 364).

Our morphological examination has revealed that two species previously included within Callerya share a suite of characters with Whitfordiodendron scandens. The most notable characters are: a) the flowers borne on extremely short pedicels $0.5-3 \mathrm{~mm}$ long vs. (2-)3-8 $\mathrm{mm}$ long in Callerya s.str.; b) the inflated, ovoid, rugose to ridged or ruminate pods with 1-3 seeds (if more than one-seeded then these often becoming fused together, Fig. 2P-Q) vs. pods flattened or if inflated then convex around seeds and contracted between them, the seeds being separate in the pod in Callerya s.str.; and c) most significantly, the sericeous keel petals which are particularly densely hairy along their lower margins (keel glabrous in Callerya s.str., see Table 4).

Based on nrDNA ITS sequence data, Li et al. (2014) showed that Callerya eriantha, C. scandens and C. nieuwenhuisii formed a clade with BS (100\%) which is sister to C. eurybotrya and C. reticulata.

\section{Adinobotrys Dunn, Bull. Misc. Inform. Kew 1911(4): 194 (1911)}

The genus Adinobotrys was described by Dunn (1911: 194) in comparison to Millettia and Padbruggea with the statement:

"affinis Millettieae Wight et Arn. sed ovario stipitato, legumine monospermo indehiscente differt [related to Millettiae Wight \& Arn. but differs by having a stipitate ovary and indehiscent one-seeded pod]".

Dunn made a further distinction between Padbruggea (which he understood to comprise P. dasyphylla and P. maingayi) and Adinobotrys, stating that the inflorescence in Padbruggea was lax and that Padbruggea lacked any appendages on the wings and keel petals (Dunn 1911: 197). Dunn (1911) included five species in Adinobotrys without assigning any one of them as the type species; $A$. erianthus (Benth.) Dunn, $A$. filipes Dunn (see above), A. nieuwenhuisii (J.J.Sm.) Dunn, $A$. myrianthus Dunn and $A$. atropurpureus (Wall.) Dunn. Geesink (1984: 83) typified Adinobotrys on the species $A$. atropurpureus, the only species of the five which is a tree and not a liana. The following year Dunn (1912b) added A. scandens (Elmer) Dunn in the belief that Elmer (1910) 
had not validly published the name under Whitfordiodendron. Dunn, recognising the uncertainty of the validity of Whitfordiodendron, validated Elmer's names firstly in Whitfordiodendron (including W. scandens) and then into his new genus Adinobotrys (Dunn 1912b: 364, 365).

Schot (1994) included A. atropurpureus, A. erianthus and A. nieuwenhuisii in Callerya and recognized $A$. myrianthus as conspecific with $C$. nieuwenhuisii. Schot did not include A. filipes in Callerya as she treated the species as belonging in Afgekia (Schot 1994: 3).

Adinobotrys has several morphological characters that separate it from the other allied genera within the Callerya group; trees vs. lianas, stipules $2-4 \mathrm{~mm}$ long, floral bracts short, c.1-3 mm long, standard petal glabrous (although this is not unique to Adinobotrys), pods inflated with glabrous, rugose surfaces and large ovoid seeds with short elliptic or circular hila (see Geesink 1984: 64 Pl. 2, 14).

Results from sequence data of nuclear ITS and chloroplast matK showed that Callerya atropurpurea was placed sister to the rest of the Callerya group (Li et al. 2014).

Callerya group taxonomy 2: Additional genera within the Callerya group as defined in treatments prior to this study.

\section{Sarcodum Lour., Fl. Cochinch. 2: 462 (1790)}

The Portuguese Jesuit missionary and botanist João de Loureiro was the first to describe the genus Sarcodum in 1790 based on its seed pods which are fleshy when young [sarcos = Gk fleshy] (Plate 2G). The type species S. scandens was described as having rose-coloured flowers in simple spikes and was found growing in woods of Cochinchina, i.e. modern day Vietnam. It has since been collected in China on Hainan Island, in Indonesia and in the Philippines. In 2017 it was discovered by co-author S. Mattapha in Bolikhamxai Province in Laos. Sarcodum scandens has standard petals 10-13 $\times 6-8 \mathrm{~mm}$ and leaves with 17-45 leaflets. Two further species have recently been described; S. bicolor in 1999 with standard petals $13 \times 8 \mathrm{~mm}$ and leaves with 9-15 leaflets from Sumba in the Lesser Sunda islands of Indonesia and S. solomonensis in 2008 from the Solomon Islands with standard petals $6 \times 5 \mathrm{~mm}$ and leaves with 17-27 leaflets (Clark 2008: 156, Table 4).

Distinguishing characters are the many small sericeous, elliptic leaflets; long stipules; long caudate floral bracts; racemose inflorescences; flowers with campanulate calyces and five very short, acute teeth; glabrous standard petals with boss callosities, elongating persistent styles on the developing pods post anthesis, and fleshy, cylindrical, botuliform fruits becoming hard when mature. The glabrous exocarp dries to dehisce from the septate tan-coloured chartaceous endocarp in which lie the 4-10 ellipsoid to reniform seeds (Kirkbride et al. 2003, Table 4).

None of the three species of Sarcodum have been included in any DNA based phylogeny prior to this study, although its affinities with other genera in the Callerya group have been postulated (Geesink 1984; Lavin et al. 1998). 


\section{Endosamara R.Geesink Leiden Bot. Ser. 8: 93 (1984)}

The unique monospecific genus Endosamara has never been included in Callerya but is nevertheless considered to be a close relative (Geesink 1984: 94). The Scottish surgeon and botanist William Roxburgh spent several decades in India where he described Robinia racemosa, a climbing shrub from the Circar Mountains [Eastern Ghats] north of Madras, which had rose-coloured flowers in what he described as racemes but we now know to be panicles (Roxburgh 1832: 329). The species was introduced to the Calcutta Botanic Garden by Henry Colebrooke in 1803 from the Coromandel region of south-east India (Roxburgh 1814: 56). The single species has a widespread distribution across India, Laos, Myanmar, Philippines, Sri Lanka and Thailand. It was placed in Millettia by Bentham (1853) and was later recombined in Wisteria (Dalzell in Dalzell and Gibson 1861: 61). Dunn (1912a: 135) placed Millettia racemosa in his monotypic Sect. Bracteatae, a taxon which was later recognised as Endosamara racemosa (Roxb.) Geesink (Geesink 1984: 93). Geesink recognised that the plant had some unusual characters (Table 4) that separated it from the genera in which it had previously been placed, notably that each of the 4 to 5 ellipsoid seeds is covered in a wing-like papery endocarp forming samaroid loments that enables the wind dispersal of each seed (see Plate 1B). The condition where a thin inner membranous layer surrounds the seeds forming loments is very rare among legume genera but is found in some species of the genus Sesbania Adans., Entada Adans. and in Plathymenia Benth. (Lavin and Schrire 2005: 452; Kirkbride et al. 2003: 332).

Chloroplast DNA data from the $r b c L$ gene (Hu and Chang 2003), showed that Endosamara racemosa was sister to Millettia japonica (Wisteriopsis japonica) with BS (82\%).

\section{Afgekia Craib, Bull. Misc. Inform. Kew 1927(9): 376 (1927)}

This genus was named by Craib (1927) for Dr Arthur Francis George Kerr, the Irish botanist who collected widely in Siam [Thailand] from 1902-1932. Kerr's Thai associate Anuwat collected the type specimen of A. sericea in Korat [now called Nakhon Ratchasima Province].

Previously, three species were recognised in the genus; A. mahidoliae (Plate 2A, E) from Kanchanaburi Province, west Thailand; $A$. sericea (Plate 2B) from north-east Thailand and $A$. filipes (Plate $2 \mathrm{H}, \mathrm{I}$ ). The latter is a much more robust climber from southern China, Laos, Myanmar, Thailand and Vietnam with panicles bearing robust and thickened inflorescence axes and fragrant pale to dark bluish-lilac flowers enclosed by caducous, broad, floral bracts. This species is fully discussed above and recombined in Padbruggea. The range of both A. mahidoliae and A. sericea is now known to extend into Laos and Vietnam in regions bordering Thailand (Lôc and Vidal 2001: 13, 14).

Afgekia (without $A$. filipes) has several distinguishing generic characters (Tables 4, 5): stipules 10-25 mm long (the longest by far in the Callerya group); racemes axillary or terminal (panicles in A. filipes); odourless flowers (fragrant in A. filipes); callosities in two pairs on the standard petal, (a unique character in the Callerya group); stamens 
with a distinctive ring of retrorse hairs on the filament immediately below the anthers and seeds with hila 15-30 mm long (Table 5).

In their analysis of $r b c L$ sequence data, Hu and Chang (2003) found Afgekia sericea placed sister to the two Wisteria species sampled. Chloroplast matK sequence data of nine species of Callerya s.l., Afgekia sericea and 11 samples from four taxa of Wisteria (Li et al. 2014) revealed, however, that Afgekia sericea was placed sister to a clade comprising Callerya s.str. This clade in turn was sister to another containing all the Wisteria samples and the two Australasian species Callerya megasperma and C. australis. Afgekia filipes was not included in this analysis. In the same paper, Li et al. (2014) also published their results from analyses of the nuclear DNA ITS spacer region. Those results showed that 14 samples of five taxa of Wisteria formed a discrete clade sister to one containing Afgekia filipes and Callerya dasyphylla sister to C. australis and C. megasperma. Afgekia sericea, however, was placed in a separate clade (with poor support) sister to Callerya eurybotrya and $C$. reticulata. In a majority consensus tree of the combined nuclear and chloroplast data Afgekia sericea is sister to Callerya eurybotrya and C. reticulata while Afgekia filipes is sister to Callerya australis and C. megasperma (Li et al. 2014).

\section{Wisteria Nutt., Gen. Amer. Pl. 2: 115 (1818)}

The genus Wisteria forms a distinct group of three species occurring in China, Japan and Korea and one, Wisteria frutescens, in the eastern USA. The latter is the most distinct on account of its later summer (vs. spring) flowering; standard petals reflexing near the middle vs. at the base in the Asian species; callosities of the ridge (vs. papillate) type, broad wing petals which arch above the keel with the tips adherent to each other enclosing the keel and covering the staminal column prior to anthesis vs. adherent to the keel and not as above, and the straight, non-septate, externally smooth pods containing reniform seeds vs. subseptate, velutinous, gently torulose pods containing lenticular seeds (Table 4, Plate 3F).

The analyses using plastid mat $K$ and nuclear ITS sequence data discussed above under Afgekia (Hu et al. 2002, Li et al. 2014), are the only DNA based studies to have sampled all four species of the genus Wisteria. Hu et al. (2002) included nine species of Callerya and Afgekia filipes while Li et al. (2014) included 15 species of the Callerya group. The other genera within the Callerya group, however, i.e. Endosamara, Sarcodum and Wisteria/Millettia japonica were excluded in these analyses.

\section{Callerya group taxonomy 3: New genera within the Callerya group as delimited in} this study

Our research has confirmed the uniqueness of other taxa within the Callerya group (Tables 1, 2). Schot noted the affinities between Endosamara racemosa and Callerya kityana in her revision of Callerya (Schot 1994: 25). Our results confirm that Callerya kityana with its sigmoid wing petals, among other unique autapomorphies, belongs in 
our new monospecific genus Sigmoidala (Fig. 3, Plate 1C-D). Our results have also revealed that two recently described Thai species, Callerya chlorantha and C. tenasserimensis (Sirichimorn et al. 2016) unequivocally share a suite of synapomorphies (Table 4) that segregate them from Callerya s.str. and they belong together in our new genus Kanburia (Plate 1E-F).

Dunn also recognised the distinctiveness of the three Australasian species $M$. australis, M. megasperma and M. pilipes which comprised his Sect. Austromillettia (Dunn 1912a: 140). Our results confirm Dunn's recognition and that these all belong in our new genus Austrocallerya (Fig. 6 and distinguishing characters, Table 4).

Based on a sampling of the morphologically most distinctive and apparently isolated taxon Callerya strobilifera Schot (which has not been sampled before in previous analyses), this species is placed here in the new genus Serawaia.

Dunn (1912a: 135, 139) in his earlier revision of Millettia, placed Millettia japonica, $M$. championii, $M$. reticulata, $M$. eurybotrya, $M$. speciosa and $M$. fordii in his Sect. Eurybotreae Dunn. Schot (1994) transferred all these species (except M. japonica) into Callerya and they all form a strongly supported clade in our analyses underpinned by shared morphological synapomorphies (Fig. 1, Table 4). Two of these species Callerya fordii and C. speciosa share gibbosities and glabrous standards with the other species in Dunn's Sect. Eurybotryeae but they differ in their densely pubescent ovaries and larger flowers. These two species are recognised here in the new genus Nanhaia (Fig. 4).

Geesink recognised the distinctive nature of Millettia japonica [Wisteria japonica] when he included it in a separate couplet in his key to the genera of Millettieae (Geesink 1984: 72). Millettia japonica is the only species that has not formally been treated as belonging to the Callerya group although it has been included in various molecular analyses as either Millettia japonica or Wisteria japonica (Doyle et al. 1997, 2000; Kajita et al. 2001; $\mathrm{Hu}$ and Chang 2003). This is now the type species of our new genus Wisteriopsis which comprises the remaining species from Dunn's Sect. Eurybotreae (Fig. 5, Plate 3A-E).

Wisteria japonica Siebold \& Zucc. has been recognised as distinct since the early days of European interest in Japanese botany (Fig. 5, Plate 3A-D). It was known to the physician Englebert Kaempfer who made a note of the species in the late $17^{\text {th }}$ century (Compton and Lack 2012). It was first validly described by Philipp von Siebold and Gerhard Zuccarini in their illustrated work Flora Japonica (Siebold and Zuccarini 1839: 88) based on material Siebold had collected near Nagasaki prior to 1829 (Compton and Thijsse 2013).

The American botanist Asa Gray recombined Wisteria japonica into Millettia (Gray 1858: 386) with no additional descriptive information other than "found on Kiu-siu belongs to a more southern Asiatic type" and "this is truly a Millettia". Dunn, however, in his revision of Millettia stated that there were several characters that allied the species with Wisteria, notably the deciduous, pinnate leaves and presence of what he considered to be large paniculate inflorescences of spreading to pendulous axillary racemes (Dunn 1912a: 153). Dunn also noted that there were other characters that separated the species from Wisteria, the occurrence of truly paniculate inflorescences, persistent bracts, and he asserted that the stamens of $M$. japonica were monadelphous (Dunn 
1912a: 153). This taxon has also been accepted as belonging in Millettia in floristic works (Ohwi 1984) and in the horticultural literature (Valder 1995) and was recently maintained in Millettia in a genetic marker study (Kim et al. 2013).

Geesink (1984) placed Millettia japonica close to his south-east Asian monotypic genus Endosamara racemosa in a couplet in his key to Tribe Millettieae based on their sharing paniculate inflorescences and what he perceived to have been an absence of callosities on the standard petal (see Table 4). Geesink also recognised that these two differed from each other by the uniquely compartmented and winged fruits in Endosamara and the presence of persistent bracteoles in $M$. japonica. He also linked $M$. japonica to his descriptions of Wisteria, Callerya and Sarcodum (Geesink 1984: 72, 93, 122). He considered M. japonica to be closest to, or included within, Callerya and specifically noted "the wings free from the keel in Millettia japonica" (Geesink 1984: 83), a character that he also noted for Wisteria (Geesink 1984: 121). In addition he recognised similarities between M. japonica and Sarcodum (Geesink 1984: 117) stating:

Sarcodum resembles Millettia japonica (which I consider to belong to Callerya) in its habit, flower characters, and in the fleshy exocarp, but in M. japonica the pod is flat and not so convex around the seeds and this species has a "true" panicle.

Schot (1994) in her revision of the genus Callerya, excluded Millettia (Wisteria) japoni$c a$ on the basis that she believed the species had slender axillary racemes rather than panicles with thickened axes and stated that she considered the species to be closest to Callerya reticulata (Schot 1994: 5). Moreover, Schot quoted Dunn (1912a: 153) in assuming that the stamens were monadelphous. This was clearly an oversight in both cases as both Callerya (sensu Schot 1994) and Millettia japonica have diadelphous stamens. Schot opined that the species "lacks the facies of a 'true' Callerya" but without further comment. Our results and observations have revealed that Callerya reticulata is indeed closely related to Wisteriopsis japonica and also belongs in Wisteriopsis (Plate 3E). In addition, our studies support the inclusion of Callerya championii, C. eurybotrya and C. kiangsiensis within Wisteriopsis.

Recent molecular phylogenies that included Wisteriopsis japonica (usually as Millettia japonica) in their analyses all used data from the chloroplast gene $r b c L$ (Doyle et al. 1997, 2000; Kajita et al. 2001; Hu and Chang 2003). Doyle et al. (1997) found that a single species of Wisteria (Wisteria sp.) and Millettia japonica were sister to each other. Doyle et al. (2000) provided data in a larger dataset combined with morphology and found that two samples of Wisteria, one Afgekia and Millettia japonica formed an unresolved clade separate from the rest of Millettia. Results from a parsimony analysis by Kajita et al. (2001) which included two species of Wisteria (W. sinensis and W. sp.), Afgekia sericea and Millettia japonica found that in their strict consensus tree, the two Wisteria samples, Millettia japonica and Afgekia sericea were unresolved in a separate (but unnamed) IRLC. $\mathrm{Hu}$ and Chang (2003) placed Millettia japonica sister to Endosamara racemosa but these were unresolved with respect to two sister samples of Wisteria, W. sinensis and W. sp.

Wisteriopsis japonica (sampled as Wisteria japonica) has also been found to possess a unique terminal $\mathrm{N}$-Acetylgalactosamine leguminous lectin which has been recognised 
to be useful as a probe for human lung squamous cell carcinoma (Soga et al. 2013). The molecular weight of the lectin in Wisteriopsis japonica which does not bind to galactose, is different to the molecular weights of the lectins of both Wisteria floribunda and W. brachybotrys and has a different sugar-binding specificity to the lectins of W. floribunda, W. brachybotrys and W. sinensis which all bind to galactose (Soga et al. 2013).

\section{Integrating our results: the Callerya group to Tribe Wisterieae}

In the most recent family-wide phylogenies of Leguminosae (Wink 2013; LPWG 2013, 2017), the IRLC is strongly supported, with the Callerya group and Glycyrrhiza placed in equivocal positions relative to the Temperate Tribe block). In the Maximum Likelihood (ML) tree of the most comprehensive phylogeny to date (LPWG 2017), Glycyrrhiza is sister to Schefflerodendron (outgroup used to root our analyses) and these are sister to a clade comprising the Callerya group + the Temperate Tribe block. In their Bayesian Inference (BI) analysis (LPWG 2017), the Callerya group and Glycyrrhiza are unresolved in a polytomy along with the Temperate Tribe block. Adinobotrys was not included in these analyses. In our combined analyses (Fig. 1, Suppl. material 1: Figs S1-S6, Table 3), Adinobotrys is placed without support as part of a grade sister to Glycyrrhiza + the rest of the Callerya group and then Glycyrrhiza, again without support, is sister to the remainder of the Callerya group. In the plastid analyses (Table 3) the positions of Adinobotrys and Glycyrrbiza are switched about in the grade and in the BI analysis, Adinobotrys + Glycyrrhiza are sister to the rest of the Callerya group, all without support. In the ITS analyses, again without support, Glycyrrhiza is sister to the entire IRLC with Adinobotrys placed sister to the remainder of the IRLC excluding the rest of the Callerya group (Table 3). The equivocal positions of Adinobotrys, the residual Callerya group and Glycyrrhiza, in all recent phylogenies, points to them being relatively isolated elements compared to the Temperate Tribe block and an understanding of the relationships between them needs further research.

As discussed earlier, Jansen et al. (2008) noted that within the IRLC, the rps12 intron was uniquely present in the Callerya group but absent in all other IRLC taxa sampled, including Glycyrrhiza. Genera in the Callerya group, that they confirmed as having the rps 12 intron, included species of Adinobotrys, Endosamara, Wisteriopsis, Afgekia, Padbruggea, Austrocallerya and Wisteria. Although Adinobotrys is supported as belonging within the Callerya group by Jansen et al. (2008) - and that superficially $A$. atropurpureus and $A$. vastus are very similar to other tropical genera within the Callerya group - we have decided to recircumscribe our emended Tribe Wisterieae to include the crown node of clades A to E (Fig 1, Suppl. material 1: Figs S1-S6, Tables 3, 4). This node is fully supported in both the combined and plastid analyses and in the ITS BI analysis, although only weakly so in the ITS ML analysis (Fig. 1, Table 3) and is diagnosed by the significant morphological synapomorphy of the lianescent habit. Adinobotrys may well belong within Tribe Wisterieae based on the evidence of Jansen et al. (2008), but on the basis of the consistent lack of support for its inclusion 
in our analyses, it is excluded here. Hu et al. (2000) also faced this quandary in that while the Phytochrome gene data (Lavin et al. 1998) and pollen data (Zhu 1994) supported linking Callerya atropurpurea with the Callerya group, their ITS and trnKI matK $K$ evidence suggested that Callerya atropurpurea was a genetic outlier with respect to Callerya. Nevertheless, Adinobotrys is treated in full in our taxonomic treatment since it is clearly a disparate element within Callerya s.l. (sensu Schot 1994) and thus, with BS (100\%) support, requires to be reinstated as a separate genus. The tree habit in Adinobotrys compared to lianas and scandent shrubs found without exception in Tribe Wisterieae serves to segregate this genus morphologically. Tribe Wisterieae together with Adinobotrys and Glycyrrhiza and the Temperate Tribe block thus represent the four main clades of the IRLC.

Tribe Wisterieae comprises a grade of three major clades (Fig. 1, Suppl. material 1: Figs S1-S6): the first branching Clade A (Sarcodum to Sigmoidala), sister to Clades B to E (Nanhaia to Wisteria) and then, Clade B (Nanhaia - Wisteriopsis) sister to Clades C-E (Callerya - Wisteria). In Clade A, the small genus Sarcodum and the monotypic Endosamara and Sigmoidala are all morphologically very distinct from each other. Sarcodum has botuliform, somewhat fleshy, pods with leaves comprising the smallest and usually most numerous leaflets in the tribe, Endosamara has unique winged and lomented seeds and Sigmoidala has distinctive 'S' shaped wing petals. Sigmoidala (as Callerya) kityana was treated as part of Callerya s.l. by Schot (1994) and is described here as a new genus. All three genera are fully supported at the generic level in the combined, plastid and ITS analyses as are all three together within Clade A except for the ITS ML and BI analyses where, with no support, the long-branched Endosamara appears attracted to other long branched genera within Clade C (Fig. 1, Table 3). The three genera together with Wisteriopsis (in Clade B) share glabrous ovaries as a unique character in the tribe; Sarcodum and Endosamara both share glabrous standards whereas the back of the standard in Sigmoidala is rufous pubescent and Sarcodum is the only one of the three with erect leafy racemes as opposed to the terminal panicles of the other two genera (Table 4). The genus Sarcodum has not been included in previous molecular phylogenies and is shown here to be fully supported as sister in the combined analyses to Endosamara and Sigmoidala.

Clade B (Fig. 1) comprises two new genera, Nanhaia and Wisteriopsis that both produce prominent gibbosities near the point of attachment of the leaf pulvinus to the stem (a character only shared otherwise by Serawaia in Clade C). Another synapomorphy shared by Nanhaia and Wisteriopsis is the presence of an annulus of hairs surrounding the calyx rim. Both genera also share glabrous standards with Endosamara and Sarcodum. Nanhaia and Wisteriopsis comprise species included within Callerya s.l. by Schot (1994), but a number of additional species in Wisteriopsis came to light as unsuspected taxa associated previously with Millettia, Wisteria and Chinese Callerya s.l. Both Nanhaia and Wisteriopsis are fully or very strongly supported as genera in all analyses except where Nanhaia is represented as a single accession (Nanhaia speciosa) in the ITS analyses (Fig. 1, Table 3) and both are fully to strongly supported together as Clade B. Moderate support values for the sister group relationship between Clade B and Clades $\mathrm{C}-\mathrm{E}$ reflect the unstable position of Clade B relative to Clade C in the ITS analyses (Table 3). 
Clade C (Fig. 1) comprises the genera Callerya s.str. (Clade C1), sister to a moderately to strongly supported alliance (in the combined analyses) of Clades C2 + C3 + C4 (Table 3), comprising Whitfordiodendron, Serawaia, Kanburia and Afgekia This grouping is poorly supported in the ITS analyses, however, and it breaks apart somewhat in the plastid analyses. Clade $\mathrm{C}$ as a whole is moderately (BS) to strongly (BPP) supported in the combined analyses but tends to break up and group with other genera in the plastid and ITS results (Table 3). The genera Afgekia, Kanburia and Whitfordiodendron are each fully supported, as is Callerya s.str. at the node above C. bonatiana. Callerya s.str., including $C$. bonatiana, is well supported in the combined and plastid BI analyses (Table 3) but only poorly so in the ML analyses. The ITS results either split Callerya s.str., placing C. bonatiana in a polytomy with the long-branched Endosamara (from Clade A2) in the ML analysis, or in an unsupported position sister to Clade B in the BI analysis. Callerya bonatiana shares the morphological synapomorphies of Callerya s.str., e.g. wings shorter than the keel, and none of the synapomorphies of other Wisterieae genera so it is treated here as part of Callerya s.str. The genus Whitfordiodendron is reinstated at generic level from Callerya s.l. (Schot 1994) and a new genus Kanburia is described from only recently collected material (Sirichamorn et al. 2016). Sister group relationships with Kanburia (Clade C3) are generally not supported, although in the ITS analyses the strongest (although still poor) support is with Serawaia (Table 3). In the combined BI analysis, however, there is strong support (0.95 BPP) for Afgekia being sister to Kanburia. The genus Serawaia is the only new genus described here that is based on a single accession since the DNA of all other material sampled was too degraded to be useful. It is a morphologically unique taxon in the Tribe Wisterieae with large cone-like strobilate inflorescences of bright yellow flowers with the persistent 15-18 $\times 8-12 \mathrm{~mm}$ bracts becoming indurate and coriaceous in fruit. No other taxon has this combination of characters and while it is placed sister to Whitfordiodendron with no support in the combined analyses and sister to Kanburia with poor support in the ITS analyses (Fig. 1, Table 3), it remains strongly supported within Clade C (BS 87\%, BPP 0.98) in the combined analyses. At the same time Serawaia cannot be placed morphologically with any other genus in Clade C. Morphologically, the presence of gibbosities below the stipules and wing petals free of the keel are unique in Clade $\mathrm{C}$ but are characteristic of all or some taxa in Clade B. No support is found, however, for links between Serawaia and clade B in these analyses.

Afgekia comprises two very distinct species separated from the other genera in the clade by a long branch and a large number of synapomorphies, most notable of which are the racemes vs. panicles present in the rest of Clade C, long sericeous floral bracts and, uniquely within the tribe, the two pairs of callosities on the standard petals (Table 4). The seeds of Afgekia have long strap-shaped hila compared to the short elliptic hila in the other genera of Clade C. Callerya is distinguished by having wing petals much shorter than the keel, Whitfordiodendron by its densely sericeous keels (glabrous elsewhere in the tribe although pubescent to tomentose in Afgekia) and Afgekia and Kanburia both lack bracteoles (present in Callerya, Serawaia and Whit- 
fordiodendron). Afgekia, Callerya, Kanburia, Serawaia and Whitfordiodendron all share densely pubescent or sericeous ovaries and backs to the standard petals. The pods of Whitfordiodendron are inflated, ovoid, rugose to ridged or ruminate (Fig. 2P), with $1-2(-3)$ seeds becoming fused together when more than one in the pod. The pods in Afgekia are inflated with densely velutinous surfaces (vs. in Callerya, Serawaia and Kanburia pods are flattened to inflated, smooth, with (1-)2-6 seeds remaining separate in the pod (Fig. 2L).

The genera Padbruggea and Austrocallerya form a strongly supported Clade D (Table 3) in the combined, plastid BI and ITS analyses although support is weak in the plastid ML analysis. Padbruggea and Austrocallerya are also strongly supported as genera in all analyses. Padbruggea is reinstated in part from Callerya s.l. (Schot 1994) and by the transfer of $P$. filipes from Afgekia. Austrocallerya is described as a new genus comprising the Australasian species in Callerya s.l. (Schot 1994). The two genera share seeds with long, strap-shaped hila and more open, laxly flowered panicles as morphological synapomorphies and are segregated from each other based on standard callosity shape, fruit characters and geographic distribution. Clade D is sister to Clade E (comprising Wisteria) with strong support in all results (Table 3) except in the plastid ML analysis.

Four species of Wisteria, three in temperate east Asia (Clade E1) and one in North America (the only non Asian-Australasian species in the Wisterieae, Clade E2) comprise Clade E with full support in the combined, plastid and ITS analyses (Table 3). Clade $\mathrm{E}$ is distinguished from Clade $\mathrm{D}$ by its deciduous vs. evergreen habit, racemose vs. paniculate inflorescences, seeds up to $12 \mathrm{~mm}$ in size in compressed pods vs. seeds larger than $12 \mathrm{~mm}$ in inflated pods and seeds with short elliptic hila vs. long strap-shaped hila (Table 4). The deciduous habit is only found otherwise in Wisteriopsis japonica.

Aberrant results in the ITS analyses compared to those derived from plastid data are thought likely to be the outcome of fewer representative taxa, probable long branch attraction in the placement of Endosamara, Kanburia, Serawaia and Afgekia and alignment problems with ITS making it difficult to ascertain true homology. The $77.1 \%$ pairwise percentage of identity across the ITS alignments vs. $92.5 \%$ for the overall plastid alignments may also be indicative of ITS being less informative than the plastid data in these analyses. It is also apparent that the two single accessions of Callerya bonatiana and Serawaia strobilifera (both limited by a lack of research material) are the most labile in the phylogeny, thereby reducing the support values of their associated clades.

\section{Taxonomic treatment of Tribe Wisterieae}

Thirteen genera within a much expanded Tribe Wisterieae are described here, encompassing five clades recovered in our phylogenetic analyses (Fig. 1). In addition, the genus Adinobotrys is fully described owing, in part, to its long association with the Callerya group but also because of its equivocal sister group relationships to tribe Wisterieae in these analyses. The following clades and included genera are listed below (Fig. 1): 


\author{
Clade A) Sarcodum, Endosamara, Sigmoidala \\ Clade B) Nanhaia, Wisteriopsis \\ Clade C) Callerya, Whitfordiodendron, Kanburia, Afgekia, Serawaia \\ Clade D) Padbruggea, Austrocallerya \\ Clade E) Wisteria
}

\title{
Emended diagnosis of Tribe Wisterieae
}

\section{Tribe Wisterieae Zhu, Cathaya 6: 115-124 (1994)}

E Subtribe Wisteriinae Endl. Gen. Pl.: 1296 (1840) [as Subtribe Wisterieae].

Type. Wisteria Nutt., Gen. Amer. Pl. 2: 115 (1818) nom. cons. 三 Glycine frutescens L., Sp. Pl. 1(2): 753 (1753).

Note. The Tribe Wisterieae is distinguished by comprising woody lianas or sprawling scandent shrubs. All species have bracts that in the most part enclose immature buds at the apex of inflorescences and all bear either true panicles or true racemes as opposed to pseudopanicles and pseudoracemes. The tribe is further distinguished from Tribe Millettieae by all genera lacking one $25 \mathrm{~kb}$ long copy of the inverted repeat in the chloroplast genome.

\section{Morphological key to the genera in Tribe Wisterieae together with Adinobotrys}

1 Inflorescences of strobilate, axillary or terminal racemes or panicles with few side axes each terminated by a strobilate bud, floral bracts $8-12 \mathrm{~mm}$ wide, persistent, imbricate, becoming indurate, coriaceous in fruit; flower bright to golden yellow

- Inflorescences never strobilate, floral bracts only as wide in Wisteria and Padbruggea where they are caducous; flowers of many colours except pure yellow......

2 Inflorescences comprising terminal leafless panicles or a combination of racemes aggregated terminally in leafy panicles ............................................... 3

- Inflorescences comprising racemes only ……........................................12

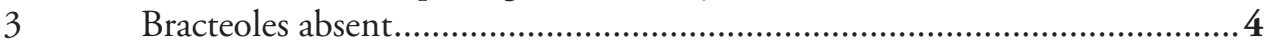

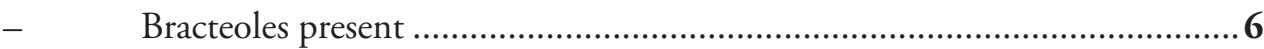

4 Seeds enclosed within lomented endocarp and dispersing as individual samaroid units at maturity; back of standard glabrous Endosamara

- $\quad$ Seeds not enclosed within papery endocarp or dispersing as individual units; back of standard pubescent 
Flowers $1.6-2 \mathrm{~cm}$ long; wing petals sigmoid, longer than keel; reflexed after anthesis; back of standard densely pubescent with ferrugineous hairs

Sigmoidala as wide

Flowers $1-1.5 \mathrm{~cm}$ long; wings \pm equalling the keel, straight and not reflexed after anthesis; back of standard sericeous with golden-brown hairs.... Kanburia Trees; back of standard glabrous Adinobotrys Lianas; back of standard glabrous, puberulent or sericeous. 7 Back of standard glabrous; prominent gibbosities present on stem below stipules

Back of standard pubescent; gibbosities absent on stem below stipules.........9 Ovary glabrous; flowers $0.7-1.6 \mathrm{~cm}$ long; stamens visible between wings and keel at anthesis

Wisteriopsis Ovary puberulent or sericeous; flowers (1.6 -)1.7-3.2 cm long; stamens enclosed within wings and keel at anthesis

Nanhaia Back of standard densely sericeous; pedicels $0.5-6(-8) \mathrm{mm}$ long; seed hilum rounded to elliptic, $1-5 \mathrm{~mm}$ long

Back of standard sparsely pubescent; pedicels (3 -)8-25 mm long; seed hilum strap-shaped, 10-40 mm long Wings \pm equalling keel in length; keel densely sericeous especially along lower margin Whitfordiodendron Callosities on standards arched on either side of midline; pods cylindrical, torulose; surfaces finely striated or smooth; seeds slightly longer than wide or \pm equal Austrocallerya Callosities on standard forming straight ridges parallel to the midline, or papillate; pods oblong-ovate; surfaces coarsely ridged; seeds \pm twice as long

Padbruggea Leaves with (9-)17-45 leaflets, these oblong-elliptic, apex obtuse, 6-12 mm wide; standard with boss callosities; pods with 4-10 seeds, botuliform, septate with fleshy exocarp, seeds 3-6 mm thick; seed hilum elliptic, $2-2.5 \mathrm{~mm}$ long

Sarcodum

- $\quad$ Leaves with 9-19 leaflets, these ovate to lanceolate, apex acute, (12 -)14-40 $\mathrm{mm}$ wide; standard with papillate or ridge callosities; pods subseptate with coriaceous exocarp

13 Plants evergreen; racemes ascending to erect; stipules 10-25 mm long; standard with two pairs of callosities, a basal papillate pair and above that a corniculate pair; pods ellipsoid with 2 seeds, seeds $8-13 \mathrm{~mm}$ thick, seed hilum strap-shaped, 15-30 mm long

- $\quad$ Plants deciduous; racemes descending to pendent; stipules 2-6 mm long; standard with a single pair of papillate or ridge callosities; pods linear with 2-5 seeds, seeds $2-6 \mathrm{~mm}$ thick, seed hilum elliptic $1-4 \mathrm{~mm}$ long... Wisteria 


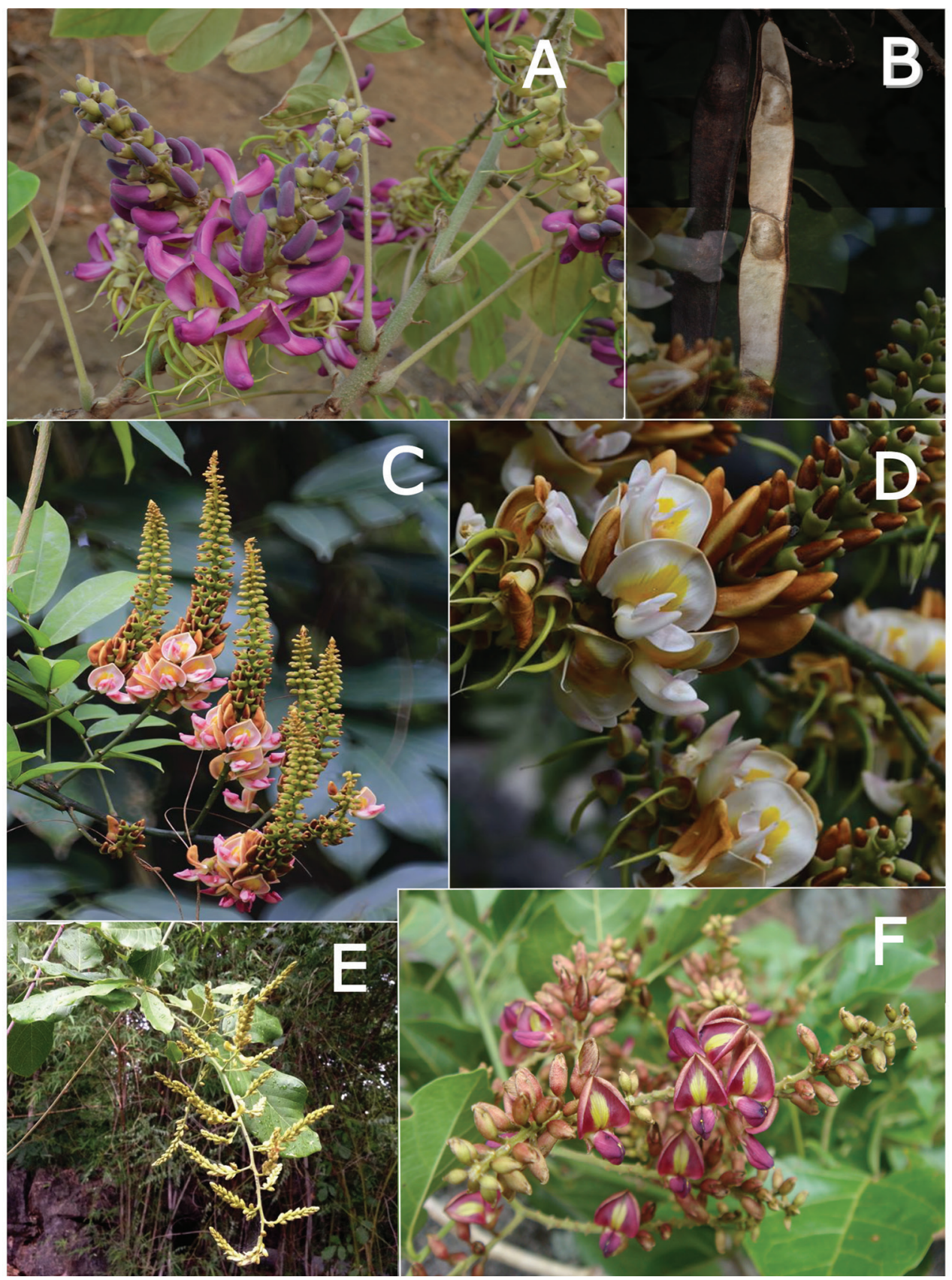

Plate I. Endosamara, Sigmoidala and Kanburia. A, B Endosamara racemosa, Thailand, Sakon Nakhon Prov., S.Mattapha s.n. C, D Sigmoidala kityana Thailand, Nan Prov. S.Mattapha 1117 E Kanburia chlorantha Thailand, Kanchanaburi Prov. Y.Sirichamorn Y2014-15-1 F Kanburia tenasserimensis Thailand, Ratchaburi, Khao Chon waterfall Y.Sirichamorn YS2015-8. 


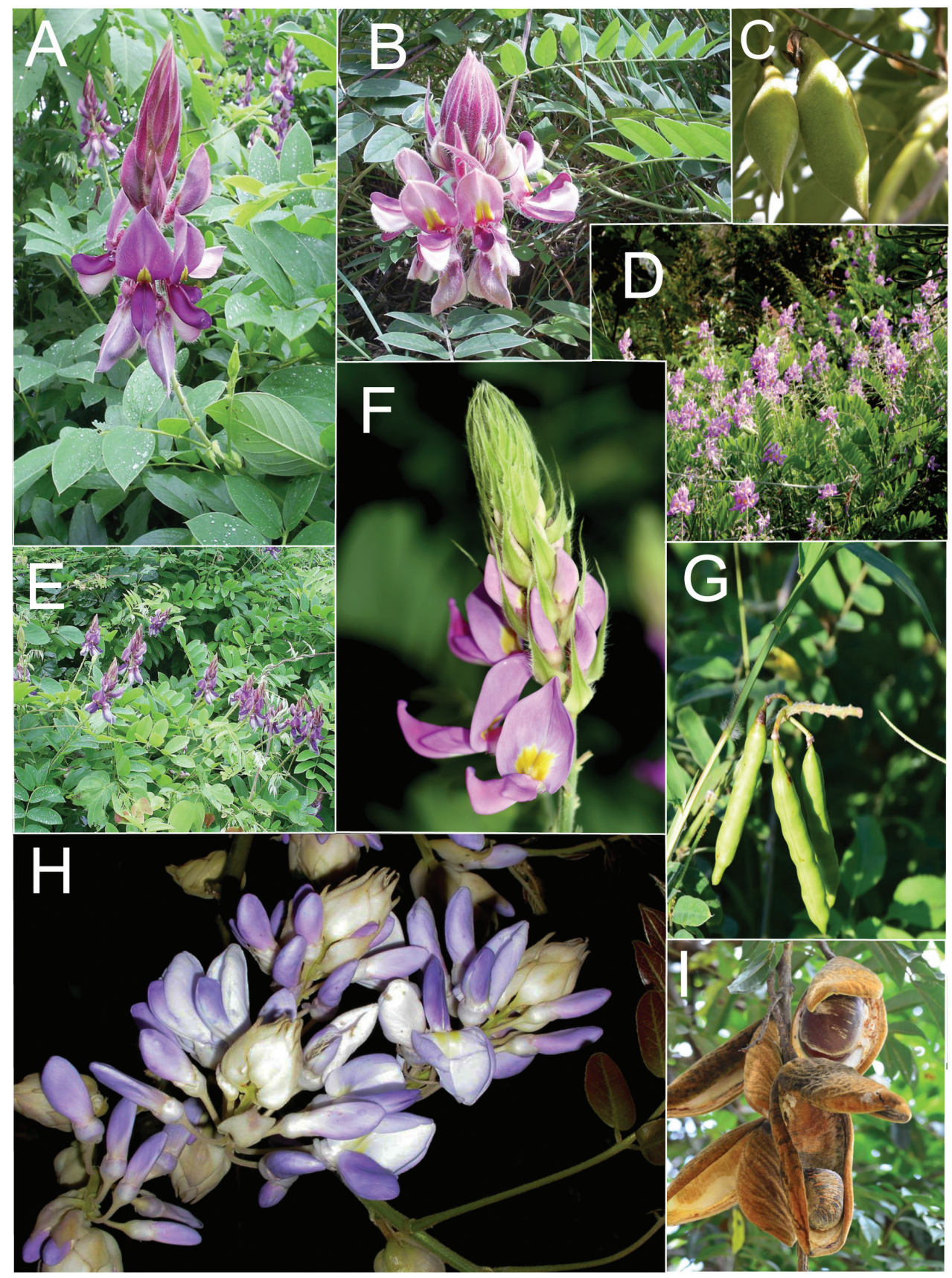

Plate 2. Afgekia, Sarcodum and Padbruggea. A Afgekia mahidoliae, Thailand, Sai Yok distr. Kanchanaburi, Y.Sirichamorn s.n. B, C Afgekia sericea Thailand S.Mattapha 1158 D Sarcodum scandens Vietnam, Quang Binh Prov. Lôc \& Quang P11554 E Afgekia mahidiliae Thailand, Sai Yok distr. Kanchanaburi Y.Sirichamorn s.n. F Sarcodum scandens Vietnam, Quang Binh Prov. Lôc \& Quang P11554 G Sarcodum scandens Laos, Sop Teuang, Bolikhamxai Prov. S.Lanorsavanh 1299 H, I Padbruggea filipes Thailand, Chiang Mai, Y.Sirichamorn \& S.Mattapha YSM2017-1. 


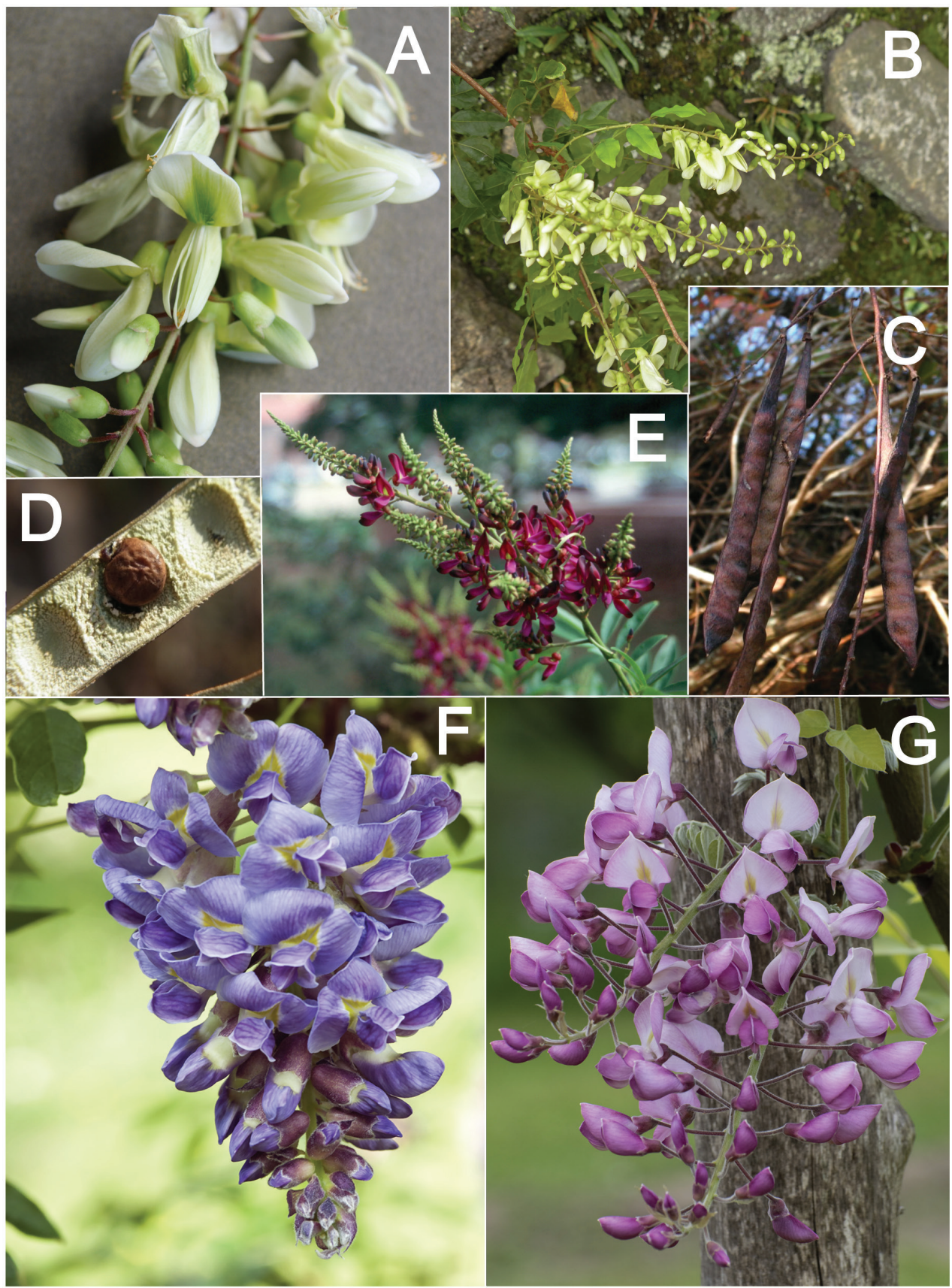

Plate 3. Wisteriopsis and Wisteria. A Wisteriopsis japonica, Cultivated, J.C.Raulston Arboretum, North Carolina 980008-17 B Wisteriopsis japonica Japan, Honshu near Kyoto G.Lewis, unvouchered C, D Wisteriopsis japonica, Cultivated, J.C.Raulston Arboretum, North Carolina 980008-17 E Wisteriopsis reticulata Cultivated, J.Compton s.n. unvouchered $\mathbf{F}$ Wisteria frutescens Cultivated, B.Schrire unvouchered G Wisteria brachybotrys cultivated, B.Schrire unvouchered. 
1. Adinobotrys Dunn, Bull. Misc. Inform. 1911: 194 (1911), emend. nov. J.Compton \& Schrire

$\equiv$ Millettia Sect. Nothomillettia Miq., Fl. Ned. Ind., Eerste Bijv. 2: 301 (1861) $\equiv$ Millettia subgen. Nothomillettia (Miq.) Kurz, J. Asiat. Soc. Bengal, Pt. 2, Nat. Hist. 45(2): 273 (1876).

Diagnosis. Adinobotrys comprises two species of evergreen trees (vs. lianes in Tribe Wisterieae). The bracteoles are persistent (caducous in Callerya s.str.), the calyx is oblique in both species, the standard is glabrous (sericeous in Callerya s.str.) and the wing petals are \pm equal to the keel in length (vs. much shorter than the keel in Callerya s.str.).

Type species. Adinobotrys atropurpureus (Wall.) Dunn 三 Pongamia atropurpurea Wall.

Genus description. Large spreading evergreen trees to $20 \mathrm{~m}$ or more in height. Stems green when young, terete, finely brown pubescent becoming brown and glabrous with age. Leaves with 5-9 (-11) leaflets, evergreen, coriaceous and nitent when mature, imparipinnate, rachis $11-33 \mathrm{~cm}$ long. Stipules $2-4 \mathrm{~mm}$ long, deltoid, persistent. Stipels absent. Leaflets 5-21 $\times 2-11 \mathrm{~cm}$, ovate, elliptic or obovate, glabrous above and below, upper surface nited, apex acute to acuminate, margins entire, base obtuse or cordate. Inflorescence a robust many-flowered erect terminal panicle 10-40 $\mathrm{cm}$ long, peduncle sparsely hairy to tomentose. Flowers 14-20 mm long, emerging from February to May (in A. atropurpureus) and May to November (in A. vastus). Floral bracts 2-4 mm long, persistent (caducous in A. vastus), ovate. Bracteoles $1-2 \mathrm{~mm}$ long, at base of calyx tube, persistent, ovate. Pedicels $2-6 \mathrm{~mm}$ long, densely pubescent. Calyx narrowly campanulate, oblique, green, tube $4-6 \times 6 \mathrm{~mm}$, puberulent externally, five lobed, lobes unequal $0.5 \mathrm{~mm}$ long, acute or obtuse. Standard 11-20 × 13-20 mm broadly ovate, inner surface pink, dark reddish-purple, rarely white, nectar guide yellow, back of standard glabrous, apex acute, callosities of boss type. Wing petals $12-19 \times 5-8 \mathrm{~mm}$, glabrous, \pm equal or longer than keel in length, each broadly semipandurate with basal claws 3-5 mm long. Keel petals 12-18 $\times 9 \mathrm{~mm}$, glabrous, apex acute to rounded. Stamens diadelphous, nine fused together, the vexillary one free, all curved upwards at apex. Ovary sparsely to densely hairy, style glabrous, 5-6 $\mathrm{mm}$ long curved upwards at apex, stigma punctate. Pods 7-25 $\times 3-6 \mathrm{~cm}$, inflated or flat $(A$. vastus), irregularly ovate to oblong or narrowly elliptic, dehiscent, surface glabrous, finely rugose, subseptate. Seeds $1-4$, irregularly ovoid to oblong or flattened orbicular, sometimes laterally compressed inside the pod, $15-38 \times 20-35 \times 3-26 \mathrm{~mm}$, hilum 2-3 $\times 2 \mathrm{~mm}$, ovate-elliptic or circular.

Etymology. adino - botrys = congested - bunch $(\mathrm{Gk})$ referring to the congested inflorescence. 


\section{Key to species of Adinobotrys}

$1 \quad$ Floral bracts $1.5-2 \mathrm{~mm}$ long; flowers $17-20 \mathrm{~mm}$ long; pod inflated, elliptic to obovate; seeds $1-2$, ovoid, 30-38 × 33-35 × 20-26 mm

A. atropurpureus

- $\quad$ Floral bracts 3-4 mm long; flowers 14-15 mm long; pod not inflated, flattened, narrowly elliptic to narrowly obovate; seeds $2-4$, flattened lenticular, $15-20 \times 25-30 \times 3-5 \mathrm{~mm}$

A. vastus

\section{Adinobotrys atropurpureus (Wall.) Dunn, Bull. Misc. Inform. 1911(4): 194 (1911)}

三 Pongamia atropurpurea Wall. Pl. As. Rar. 1(4): 70 t. 78 (1830). Type: Myanmar. "Martaban [Mottama] ad Amherst [Kyaikkami] 15 July 1827”, Wallich Cat. No. 5910, K000881026 (K, holo.!); (BO, iso.); (CAL, iso. x 2); BM000997335 (BM, iso!), P02141756 (P, iso!) 三 Millettia atropurpurea (Wall.) Benth. Pl. Jungh. [Miquel] 2: 249 (1852) E Phaseoloides atropurpurea (Wall.) Kuntze, Revis. Gen. Pl. 1: 201 (1891) 三Whiffordiodendron atropurpureum (Wall.) Dunn, Bull. Misc. Inform. Kew 1912(8): 364 (1912) 三 Callerya atropurpurea (Wall.) Schot, Blumea 39(1-2): 15 (1994).

= Millettia paniculata Miq., Fl. Ned. Ind., Eerste Bijv. 2: 301 (1861). Type: Indonesia, Sumatra "Sumatra orient. in prov. Palembang prope Kebur Lahat (T.)" 3675 H.B. Leguminosae, Masiboengan, Hortus Botanicus 023149 Utrecht, [Johannes Elias] Teijsmann s.n., U0003669 (U, holo.!).

= Padbruggea pubescens Craib, Bull. Misc. Inform. Kew 1927(2): 61 (1927) Type: Thailand, Prov. Nakawn Panom [Nakhon Phanom], Ta Uten, elev. 1200 m, 15 February 1924, tree, fls pink. Ki Mo., A.F.G.Kerr 8457, K000881016 (K, holo.!); (ABD, iso.); BM000997332 (BM, iso.!); E00275433 (E, iso.!) 三 Whitfordiodendron pubescens (Craib) Burkill, Bull. Misc. Inform. Kew 1935(5): 319 (1935) 三 Callerya atropurpurea (Wall.) Schot var. pubescens (Craib) P.K.Lôc, Bot. Zhurn. (Moscow \& Leningrad) 81(10): 98 (1996).

Illustrations. Lôc and Vidal in Fl. Cambodge, Laos \& Vietnam 30: 34, t. 8 [9-11] (2001). https://singapore.biodiversity.online (in Home Page enter Callerya atropurpurea)

Distribution. Cambodia; India; Indonesia (Java, Sumatra); Laos, Malaysia (Peninsula); Myanmar; Thailand and Vietnam.

Habitat. A component of evergreen forests from sea level to $1200 \mathrm{~m}$.

\section{Adinobotrys vastus (Kosterm.) J.Compton \& Schrire, comb. nov.} urn:lsid:ipni.org:names:77198982-1

三 Millettia vasta Kosterm., Reinwardtia 5: 349 (1960). Type: Indonesia, Kalimantan [Borneo], Belajan River near Muara Lempong, June 1956, [André Joseph Guillaume Henri] Kostermans 12516A, BO-1249898 (BO, holo.!); BM000997327 
(BM, iso.!); L0018805 (L, iso.!); K000880991 (K, iso.!); P03081895 (P, iso.!) $\equiv$ Callerya vasta (Kosterm.) Schot, Blumea 39(1-2): 36 (1994).

Distribution. Borneo: Brunei; Indonesia (Kalimantan); Malaysia (Sabah, Sarawak). Habitat. Component tree in woods and forests from sea level to $250 \mathrm{~m}$.

\section{Clade A - Sarcodum, Endosamara and Sigmoidala}

(Fig. 1; Suppl. material 1: Figs S1-S6)

\section{Sarcodum Lour., Fl. Cochinch. 2: 462 (1790)}

Diagnosis. The three species of Sarcodum are most closely allied to Endosamara and Sigmoidala but the genus is easily distinguished from the other two by the presence of bracteoles subtending the calyces (absent in Endosamara and Sigmoidala) and the smaller leaflets $(0.3-2(-2.5) \mathrm{cm}$ wide in Sarcodum vs. (2-)2.5-7 cm wide in Endosamara and Sigmoidala). Sarcodum, moreover, has leafy racemose inflorescences as opposed to the robust, erect panicles found in Endosamara and Sigmoidala. The back of the standard in Sarcodum and Endosamara is glabrous while that of Sigmoidala is densely pubescent. Sarcodum has fleshy botuliform pods that become woody on drying with oblate seeds borne in septate chambers while the seeds of Sigmoidala are flattened, ellipsoid and those of Endosamara are oblong, surrounded by a papery endocarp. The most widespread species $S$. scandens has leaves with between 17 and 45 narrowly elliptic leaflets - the most numerous in the tribe - each terminating in a short mucro.

Type species. Sarcodum scandens Lour.

Genus description. Three species of scandent twining vines scrambling over shrubs reaching 5-10 m. Stems grey-green when young, terete, densely pubescent, mature stems dark green becoming rusty brown, glabrous. Leaves with 9-45 leaflets, evergreen, often spotted with tannin deposits, sericeous when mature, imparipinnate, rachis 6-19 cm long. Stipules 3-12 mm long, linear-lanceolate, persistent. Stipels 3-6 $\mathrm{mm}$ long, linear, persistent. Leaflets $0.8-4.5 \times 0.3-2.5 \mathrm{~cm}$, elliptic, grey-green sericeous above and densely white sericeous below, apex rounded, mucronate, mucro c. 1.5-2 mm (retuse in S. bicolor), margins entire, base rounded. Inflorescence of erect leafy axillary and terminal racemes 3-12 cm long, peduncle densely silvery sericeous. Flowers 6-19 mm long, emerging from November - April. Floral bracts 6-20 mm long, caducous, densely pubescent, narrowly deltoid or ovate-deltoid apex acute or long acuminate. Bracteoles at base of calyx 2-7 mm long. Pedicels 4-12 mm long, densely pubescent. Calyx $3 \times 5 \mathrm{~mm}$, broadly campanulate, green or pink, sericeous externally, five lobed, upper 2 lobes \pm connate, lower 3 lobes $1-3 \mathrm{~mm}$ long, acute. Standard 10-13 × 6-8 mm, ovate, inner surface glabrous, pink or pinkish-lilac, nectar guide broad, dark yellow, back of standard glabrous, apex acute. Callosities of boss type. Wing petals 8-13 $\times 3 \mathrm{~mm}$, glabrous, much shorter than or subequal to the keel, each narrowly semi-pandurate, slightly curved upwards at the apex; free from the 
keel, basal claws $1-4 \mathrm{~mm}$ long. Keel petals $13 \times 4 \mathrm{~mm}$, glabrous, united into a falcate, navicular cup, apex acute and somewhat reflexed. Stamens diadelphous, nine fused together, the vexillary one free, all curved upwards at apex. Ovary glabrous, style glabrous, 3-4 mm long, curved upwards at apex, stigma punctate. Pods 3.5-5 × 0.7-1.2 $\mathrm{cm}$, green, botuliform, dehiscent, gently torulose, surface glabrous, black and hard when dry, internally septate. Seeds 4-10, ellipsoid or oblong, 5-7.5 × 3.5-5 × 2.5-4.5 $\mathrm{mm}$, oblong, rounded at each end, hilum central, broadly elliptic $2-2.5 \times 1 \mathrm{~mm}$.

Distribution. China; Indonesia; Laos; Philippines; Solomon Islands; Vietnam.

Etymology. sarcos = Gk fleshy.

Habitat. All three species are climbing and scrambling vines growing in low thicket from sea level to $300 \mathrm{~m}$.

\section{Key to species of Sarcodum}

1 Leaves with 9-15 leaflets, leaflet apices rounded or retuse; floral bracts ovatedeltoid $10 \times 4 \mathrm{~mm}$. S. bicolor

- $\quad$ Leaves with 17-45 leaflets; leaflet apices mucronate; floral bracst narrowly deltoid . .2

$2 \quad$ Floral bracts $6 \times 0.5 \mathrm{~mm}$ S. solomonensis

Floral bracts $16-30 \times 3 \mathrm{~mm}$ S. scandens

Sarcodum scandens Lour., Fl. Cochinch. 2: 462 (1790)

= Clianthus binnendyckianus Kurz, J. Asiat. Soc. Bengal Pt. 2 Nat. Hist. 40(1): 51 (1871). Type: "Moluccos [Maluku], Ceram [Seram], Cult. in Hort. Bogor ab Binnendyck", S.Binnendijk s.n., (BO, holo., not seen); K000117839 (K, iso.!).

Type. Vietnam. "In sylvis Cochinchinae, G 151 Sarkinum = Cay muong deei = Sarcodum p. 462 2-delph" J. de Loureiro or local collector, BM001209557 (BM, holo.!) $\equiv$ Clianthus scandens (Lour.) Merr., J. Bot. 66: 265 (1928)

Illustrations. Lôc and Vidal in Fl. Cambodge, Laos \& Vietnam 30: 7, t. 1 (2001); Clark in Kew Bull. 63(1): 156 (2008); Sun and Pedley FOC Illustrations 10: 175 fig. 202 [1-9] (2010). Plate 2D, Plate 2F-G.

Distribution. China (Hainan); Indonesia (Seram, Sulawesi); Laos; Philippines; Vietnam.

\section{Sarcodum bicolor Adema, Blumea 44: 407 (1999)}

Type. Indonesia, Sumba, Nusa Tengarra [Lesser Sunda Islands], "1925, Soemba”, [L.] Iboet 385, L0064653 (L, holo.!); A00104485 (A, iso.!); K000117840 (K, iso.!); (SING, iso.) 
Illustration. Clark in Kew Bull. 63(1): 159 (2008).

Distribution. Indonesia (Sumba Island).

Sarcodum solomonensis R.Clark, Kew Bull. 63(1): 155, t. 1 (2008)

Type. Solomon Islands, Gizo Island ridge top $250 \mathrm{ft}$. asl. 28 April 1970, R.Mauriasi \& collectors BSIP 18096, K000556150 (K, holo.!); L0418332 (L, iso.!).

Distribution. Solomon Islands (Gizo).

\section{Endosamara R.Geesink, Leiden Bot. Ser. 8: 93 (1984)}

$\equiv$ Millettia Sect. Bracteatae Dunn, J. Linn. Soc., Bot. 41: 135 (1912a)

Diagnosis. The monospecific Endosamara racemosa was recognised at generic level by Geesink (1984: 93) principally by its unique fruits with their seeds forming segregating loments encased in endocarp each with a flat wing, a unique feature within the tribe. Endosamara has stipules 6-12 $\mathrm{mm}$ long (vs. 3-7 mm in Sigmoidala and 1-6 $\mathrm{mm}$ in Callerya s.str.), lacks floral bracteoles, has glabrous ovaries and glabrous standard petals (vs. back of standard pubescent in Sigmoidala).

Type species. Endosamara racemosa (Roxb.) Geesink $\equiv$ Robinia racemosa Roxb.

Genus description. A robust, twining woody vine. Stems green when young, terete, pubescent, mature stems pale brown, glabrous. Leaves with 7-13 leaflets, evergreen, coriaceous when mature, imparipinnate, rachis 10-24 cm long. Stipules 6-12 mm long, narrowly lanceolate, persistent, becoming woody and spinose on old branches. Stipels 3-6 mm long, linear, persistent. Leaflets 5-13 $\times 2-7 \mathrm{~cm}$, oblongobovate, elliptic, glabrous above, tomentose below becoming glabrous, apex acute, margins entire, base obtuse to cuneate. Inflorescence a robust many-flowered terminal panicle $20-50 \mathrm{~cm}$ long, peduncle densely silvery-brown hairy. Flowers $12-16 \mathrm{~mm}$ long, emerging from March - June. Floral bracts 6-15 mm long, caducous, densely pubescent, linear-lanceolate. Bracteoles absent. Pedicels 3-6 mm long, densely pubescent. Calyx $3 \times 6 \mathrm{~mm}$ campanulate, green, densely puberulent externally, five lobed, lobes \pm equal, 1-3 mm long, broadly acute, obtuse or subtruncate, becoming more fleshy and rounded at maturity. Standard 10-15 × 12-15 mm, suborbicular or broadly ovate, inner surface pale to dark pink, pinkish purple, rarely white, nectar guide greenish yellow, back of standard glabrous, apex acute or emarginate. Callosities of boss type. Wing petals 12-13 × 3-5 mm, glabrous, slightly longer than keel, each narrowly semi-pandurate, slightly curved upwards at the apex with basal claws $3 \mathrm{~mm}$ long. Keel petals 10-12 × 4-6 mm, glabrous, united into a falcate, navicular cup, apex obtuse. Stamens diadelphous, nine fused together, the vexillary one free, all curved upwards at apex. Ovary glabrous, style glabrous, 4-5 mm long, curved upwards at apex, stigma punctate. Pods 8-25 $\times 1-2 \mathrm{~cm}$, green, flattened, linear, dehiscent, exocarp raised above the seeds, surface glabrous, black when dry, internally septate. Seeds $4-5,10-12 \times 6-8$ 
$\times 5 \mathrm{~mm}$, oblong, short beaked at one end, each seed separated inside the pod, entirely covered in a thin chartaceous layer of endocarp one side of which extends into a papery samaroid wing forming a compartmented unit that becomes free on maturity, wings $3-5 \times 1 \mathrm{~cm}$, hilum eccentric at beaked end of seed, broadly elliptic $2-3 \times 2 \mathrm{~mm}$.

\section{Endosamara racemosa (Roxb.) R.Geesink, Leiden Bot. Ser. 8: 93 (1984)}

$\equiv$ Robinia racemosa Roxb., Fl. Ind. ed. 2 vol. 3: 329 (1832)

Type. India, Andra Pradesh, Circar Mts. "Diadelphia decandria. Galuda tiga of the Gentoos", Roxburgh s.n., E00301096 (E, lecto.!, designated here); Roxburgh s.n. E00301095 (E, isolecto.!) $\equiv$ Tephrosia racemosa (Roxb.) Wight \& Arn. Prodr. Fl. Pen. Ind. Or. 1: 210 (1834) $\equiv$ Millettia racemosa (Roxb.) Benth. Pl. Jungh. [Miquel] 2: 249 (1853).

\section{Key to varieties of Endosamara racemosa}

1 Flowers with petals pink or purple var. racemosa

- $\quad$ Flowers with petals white or pale pink var. pallida

\section{Endosamara racemosa var. racemosa}

EWisteria racemosa (Roxb.) Dalzell, Bombay Flora: 61 (1861)

三 Phaseoloides [Phaseolodes] racemosum (Roxb.) Kuntze, Revis. Gen. Pl. 1: 201 (1891). = Millettia leiogyna Kurz, J. Asiat. Soc. Bengal, Pt. 2, Nat. Hist. 42(2): 67 (1873). Type: Burma, [Myanmar] "Kurz, Martaban [Mottama], in an upper mixed forest at Nakawa Choung, Toukyeghat east of Tounghoo. Fl. April” not found. Type: Upper Burma [Myanmar] Southern Shan State, Toungyi [Taunggyi], comm. Dr [George] King June 1896, 1894, Abdul Khalil s.n. (K, neo.!, designated here) $\equiv$ Phaseoloides [Phaseolodes] leiogynum (Kurz) Kuntze, Revis. Gen. Pl. 1: 201 (1891).

Illustration. Lôc and Vidal in Fl. Cambodge, Laos \& Vietnam 30: 16, t. 3 (2001); Plate 1A, B.

Distribution. India; Laos; Myanmar; Malaysia (Peninsula); Philippines; Thailand; Vietnam.

Etymology. The generic name combines endo (endocarp) and samara (the remarkable samaroid winged seeds).

Habitat. In dry woods, thickets and forest margins from sea level to $850 \mathrm{~m}$. climbing over rocks, on banks and among scrub and trees. 
Endosamara racemosa var. pallida (Dalzell) J.Compton \& Schrire, comb. nov. urn:Isid:ipni.org:names:77199032-1

三Wisteria pallida Dalzell, Bombay Flora: 61 (1861). Type: [Icon] India, "In the Dangs,

Wassoorna forest, Bombay, very rare, Dr [Alexander] Gibson” (lecto.!, designated here) $\equiv$ Millettia pallida (Dalzell) Dalzell, J. Linn. Soc., Bot. 13: 187 (1873)

Nomenclatural note. This plate by an unknown artist at the Bombay Botanic Garden at Dapuri, commissioned by Nicol Alexander Dalzell, has the annotation "comm. N. Dalzell 1/72 [January 1872], Wisteria pallida Dalz. corrected to Millettia pallida Dalz. mss." in Dalzell's hand. It is numbered 18 among Dalzell's artworks in J. D. Hooker's collections at $\mathrm{K}$ and represents the white or pale creamy-yellowish flowered form of this species in western India. It is recombined by us here as var. pallida. Another illustration representing this taxon is of a plant that was cultivated at the Madras AgriHorticultural Garden. The plant was collected from the Rammanmally [Sandur] Hills, Karnataka by the garden's superintendent Colonel Francis Archibald Reid and later painted by the artist P. Mooregasan Moodeliar in July 1853. RBGE CAH 27 (Cleghorn Collection, see Noltie 2016: 43)

Illustration. As Millettia racemosa http://florakarnataka.ces.iisc.ac.in/hjcb2/herbsheet.php?id=2056\&cat $=1$

\section{Sigmoidala J.Compton \& Schrire gen. nov. urn:Isid:ipni.org:names:77198973-1}

Diagnosis. The monospecific Sigmoidala kityana has several affinities with Endosamara racemosa including the absence of bracteoles and glabrous ovaries, characters which also separate it from Callerya s.str. which has bracteoles and sericeous ovaries. Sigmoidala also shares with Endosamara the pubescent floral bracts and broadly campanulate, slightly oblique, subtruncate calyx as noted by Schot (1994: 25) but it was placed in Callerya on account of the fruits that lacked the lomented endocarp. The stipules in Sigmoidala are shorter, 3-7 mm long (vs. 9-12 mm in Endosamara); pedicels shorter, 3-4 mm long (vs. 4-12 mm in Endosamara and Sarcodum), floral bracts linear, 6-8 mm long (vs. linear-lanceolate, 8-12 mm long in Endosamara, 6-20 mm in Sarcodum); the back of the standard densely, appressed rufous pubescent (vs. glabrous in Endosamara and Sarcodum) and the wing petals of Sigmoidala are unique within Tribe Wisterieae being a sigmoid shape, reflexed at the midpoint and extending outwards towards the apex (see Fig. 3I). The pods of Sigmoidala are flattened, linear to obovate, $7-11 \times 1-2 \mathrm{~cm}$ (vs. septate, flattened, linear, $10-25 \times 1-2 \mathrm{~cm}$ in Endosamara and botuliform in Sarcodum).

Type species. Sigmoidala kityana (Craib) J.Compton \& Schrire $\equiv$ Millettia kityana Craib. 
Genus description. A robust, twining woody vine. Stems very dark green when young, terete. Leaves evergreen, coriaceous and nitid when mature, imparipinnate with 7-9 (-11) leaflets, rachis 12-30 cm long. Stipules 3-7 mm long, narrowly deltoid, persistent. Stipels 3-6 mm long, linear, persistent. Leaflets $7-18 \times 2-5 \mathrm{~cm}$, elliptic to narrowly obovate, glabrous above and below, apex cuspidate, margins entire, base cordate. Inflorescence a robust many-flowered terminal panicle $20-50 \mathrm{~cm}$ long, peduncle sparsely hairy. Flowers 16-20 mm long, emerging from August - November. Floral bracts 6-8 $\mathrm{mm}$ long, caducous, linear. Bracteoles absent. Pedicels 3-4 mm long, glabrous. Calyx $4 \times$ $6 \mathrm{~mm}$, campanulate, green, densely puberulent externally, five lobed, lobes \pm equal 1-6 $\mathrm{mm}$ long, rounded, obtuse or subtruncate becoming subentire after anthesis. Standard 10-12 × 12-13 mm, suborbicular, inner surface white with a pink flush, nectar guide broad, deep golden-yellow, back of standard densely appressed, ferrugineous or rufous pubescent, apex acute or emarginate. Callosities of boss type. Wing petals glabrous, longer than keel in length but notably sigmoid towards apex and thereby shortened, each narrowly semi-pandurate 10-14 × $3 \mathrm{~mm}$ with basal claws 1-3 mm long. Keel petals 10-12 × 4-6 mm, glabrous, united into a long navicular cup, apex acute. Stamens diadelphous, nine fused together, the vexillary one free, all curved upwards at apex. Ovary glabrous, style glabrous, $3 \mathrm{~mm}$ long, curved upwards at apex, stigma punctate. Pods 7-11 × 1-2 cm, flattened, linear to narrowly obovate, dehiscent, exocarp surface glabrous, speckled with small pustules, subseptate. Seeds 1-5(-8), ellipsoid or orbicular $12-14 \times 12-13 \times 13$ mm, hilum 1.6-2 × 2 mm, elliptic. Fig. 3C-D.

\section{Sigmoidala kityana (Craib) J.Compton \& Schrire, comb. nov.} urn:Isid:ipni.org:names:77198984-1

三 Millettia kityana Craib, Bull. Misc. Inform. Kew 1927(2): 58 (1927). Type: Thailand, "coll. AFG Kerr, locality Chiengmai [Chiang Mai], altitude 300 m. Aug. 23 1914, large woody climber, flowers pink, by village", Kerr 3347, K000881009 (K, lecto.! designated here); ABDUH: 2/213 (ABD, isolecto.); K000881010 (K, isolecto.!); BM000997330 (BM, isolecto.!); TCD0015789 (TCD, isolecto.!) 三 Callerya kityana (Craib) Schot, Blumea 39(1-2): 24. (1994)

Note. In the key to the species of Callerya, Schot placed this species within the segregating couplet "stipellae persistent" as opposed to "stipellae caducous" and noted that the bracteoles were absent and that the wing petals were longer than the keel (Schot 1994: 9). In our study we have found that the persistent or caducous nature of the stipels is not particularly significant and, moreover, is frequently difficult to verify. Schot also recorded in her species description that the stipules were 3-4 $\mathrm{mm}$ long even though Craib had stated that they were 6-8 mm long (Craib 1927: 58). Our observations have confirmed that the stipules rarely exceed $7 \mathrm{~mm}$ in length. This very distinct monospecific genus occurs only within a narrow region of northern and north-eastern Thailand (Fig 3, Plate 1C, D). 


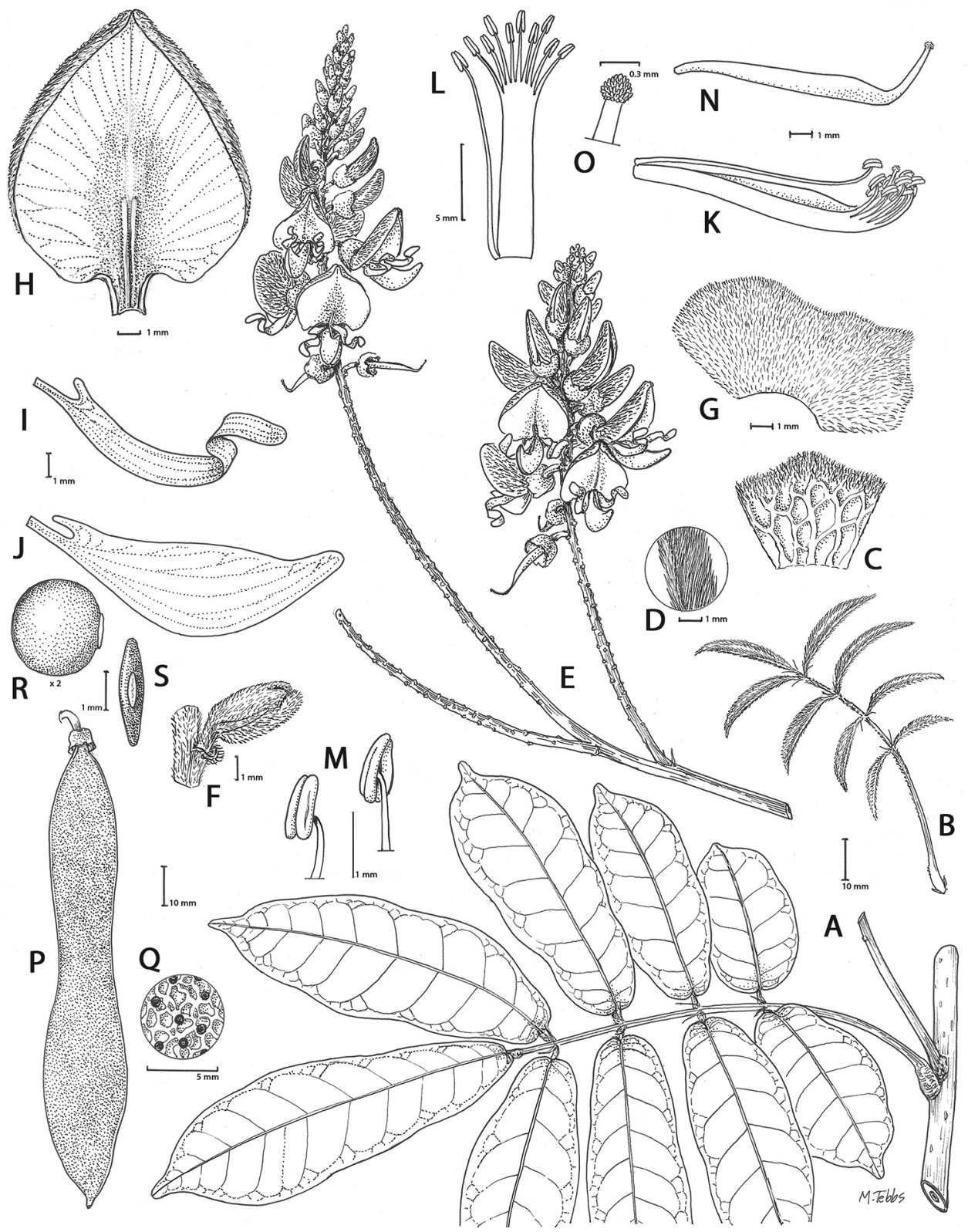

Figure 3. Sigmoidala kityana (Craib) J.Compton \& Schrire. A Habit B young leaf C lower surface of leaf $\mathbf{D}$ leaflet detail of hairs $\mathbf{E}$ inflorescence $\mathbf{F}$ flower bud with bracteole and pedicel $\mathbf{G}$ calyx external surface $\mathbf{H}$ standard petal inner surface $\mathbf{I}$ wing petal $\mathbf{J}$ keel petal $\mathbf{K}$ staminal column lateral view $\mathbf{L}$ staminal column ventral view $\mathbf{M}$ stamen dorsal and ventral views $\mathbf{N}$ ovary lateral view $\mathbf{O}$ style and stigma $\mathbf{P}$ pod $\mathbf{Q}$ pod detail of surface $\mathbf{R}$ seed ventral view $\mathbf{S}$ seed lateral view (all from Clark 245). Drawn by Margaret Tebbs. 
Illustration. (as Millettia kityana) http://crassa.cocolog-nifty.com/blog/2015/04/ millettia-kitya.html

Distribution. Northern Thailand: Chiang Mai, Nan, Lamphun, Sukhothai; North-east Thailand: Loei.

Etymology. The generic name refers to the remarkable sigmoid wing petals.

Habitat. Climbing among dry forest trees in partial sunlight to $400 \mathrm{~m}$. elevation.

\section{Clade B - Nanhaia and Wisteriopsis}

(Fig. 1; Suppl. material 1: Figs S1-S6)

\section{Nanhaia J.Compton \& Schrire, gen. nov. urn:Isid:ipni.org:names:77198974-1}

$\equiv$ Millettia Sect. Corynecarpae Z.Wei, Acta Phytotax. Sin. 23(4): 281 (1985)

Diagnosis. Nanhaia, with two species, is readily distinguished from Wisteriopsis by the densely pubescent or sericeous ovaries (glabrous in Wisteriopsis) and the larger flowers frequently 15-35 mm long (vs Wisteriopsis 7-15 mm long). In Nanhaia the stipules arise immediately above the swollen, hardened gibbosities (Fig. 4).

Type species. Nanhaia speciosa (Champ. ex Benth.) J.Compton \& Schrire $\equiv$ Millettia speciosa Champ. ex Benth.

Genus description. Procumbent or scandent twining vines, $1-5 \mathrm{~m}$ high, scrambling among rocks and scrub. Stems green or brown, terete, pubescent. Leaves with 5-17 leaflets, evergreen, glabrous or with a few scattered hairs below, imparipinnate, rachis 3-30 cm long. Stipules 2-4 mm long, linear or deltoid, caducous in N. fordii (persistent N. speciosa). Stipels 1-3 mm long, linear, persistent. Leaflets 3-9 $1-4 \mathrm{~cm}$, ovate-elliptic or narrowly elliptic, glabrescent or sparsely hairy, apex acuminate or cuspidate, margins entire, base rounded to subcordate. Inflorescence erect or pendant sometimes leafy panicles 4-20 cm long, frequently comprising several leafy lateral racemes, peduncle yellow tomentose or densely brown pubescent. Flowers $16-32 \mathrm{~mm}$ long, emerging from June to September. Floral bracts 3-7 mm long, linear or narrowly deltoid, persistent (caducous in $N$. fordii). Bracteoles at base of calyx 1-5 mm long, narrowly ovate or elliptic, persistent. Pedicels 4-11 mm long, glabrous or pubescent. Calyx 4-6 $\times 5-9$ mm campanulate, oblique, pubescent externally, (densely pubescent internally on $N$. fordii) five lobed, teeth unequal, 1-3 mm long, acute. Standard 12-18 × 11-18 mm, suborbicular, white, cream or pink, nectar guide pale or dark green, back of standard glabrous, apex acute or obtuse. Callosities of boss type. Wing petals 12-17 × 4-6 mm, glabrous, subequal to the keel, each narrowly semi-pandurate, slightly curved upwards at the apex; free from the keel, apex obtuse, basal claws $2-5 \mathrm{~mm}$ long. Keel petals 12-16 $\times$ 4-6 mm, glabrous, united into a falcate, navicular cup, apex obtuse, basal claw 4-9 $\mathrm{mm}$ long. Stamens diadelphous, nine fused together, the vexillary one free, all curved upwards at apex. Ovary densely sericeous, especially along thickened margins, style 
ciliate ( $N$. speciosa) or glabrous ( $N$. fordii), 2-3 mm long curved upwards at apex, stigma punctate. Pods 10-20 × 1-2 cm, flat, linear, dehiscent, surface pubescent to densely brown tomentose, brown and hard when dry, subseptate. Seeds 2-10, ovoid or ellipsoid, 10-12 × 5-12 × 1-7 mm, hilum terminal or central, elliptic, 2-3 × $1 \mathrm{~mm}$. Fig. 4 .

Distribution. China (Fujian, Guangdong, Guangxi, Guizhou, Hainan, Hunan, Yunnan); Vietnam (north).

Etymology. Nanhai is the Chinese name for the South China Sea which links southern China with Vietnam.

\section{Key to species of Nanhaia}

2 Flowers 15-19 mm long; bracts 3-4 mm wide, narrowly deltoid, caducous; leaves $5-7$ foliolate............................................................ fordii

- $\quad$ Flowers 20-32 mm long; bracts 4-7 mm wide, deltoid-lanceolate, persistent; leaves $7-17$ foliolate.

N. speciosa

Nanhaia speciosa (Champ. ex Benth.) J.Compton \& Schrire, comb. nov. urn:Isid:ipni.org:names:77198985-1

三 Millettia speciosa Champ. ex Benth., Hooker’s J. Bot. Kew Gard. Misc. 4: 73 (1852).

Type: China, "Hong Kong, Millett 505”, K000881029 (K, lecto.!, designated by

Schot (1994) but see note below)

三 Phaseoloides [Phaseolodes] speciosa (Champ. ex. Benth.) Kuntze, Revis. Gen. Pl. 1: 201 (1891)

三 Callerya speciosa (Champ. ex Benth.) Schot, Blumea 39(1-2): 32 (1994)

Nomenclatural note. Schot selected a specimen at K, Champion 505 as lectotype for the name Millettia speciosa Champ. ex Benth. (Schot 1994: 32). This was, however, clearly an error because another specimen, Millett 505, was cited in the protologue and determined as type by Dunn's annotation on the sheet at $\mathrm{K}$ prior to the publication of his monograph on Millettia in 1912. Dunn had incorrectly cited the specimen as Champion 505 and not Millett 505 which may explain Schot's error (Dunn 1912a: 155). Bentham's paper on the plants of Hong Kong was based on the collections of Major John George Champion (1815-1854). There are two specimens at K collected by Champion in the Hooker Herbarium: K000881027 and K000881028. Both are annotated "Champion 261, Hong Kong", but these specimens were not specifically cited by Bentham in the protologue which merely stated "on Victoria Peak" [on Hong Kong island] and are therefore regarded as uncited specimens that comprise part of the original material associated with the name (Art. 9.4(a)). The Millett collection K000881029 is annotated " 505 Millettia grandiflora sp. n. fls white and yellow Hong Kong" and is dated 1854 in Bentham's Herbarium. This collection was cited in the pro- 


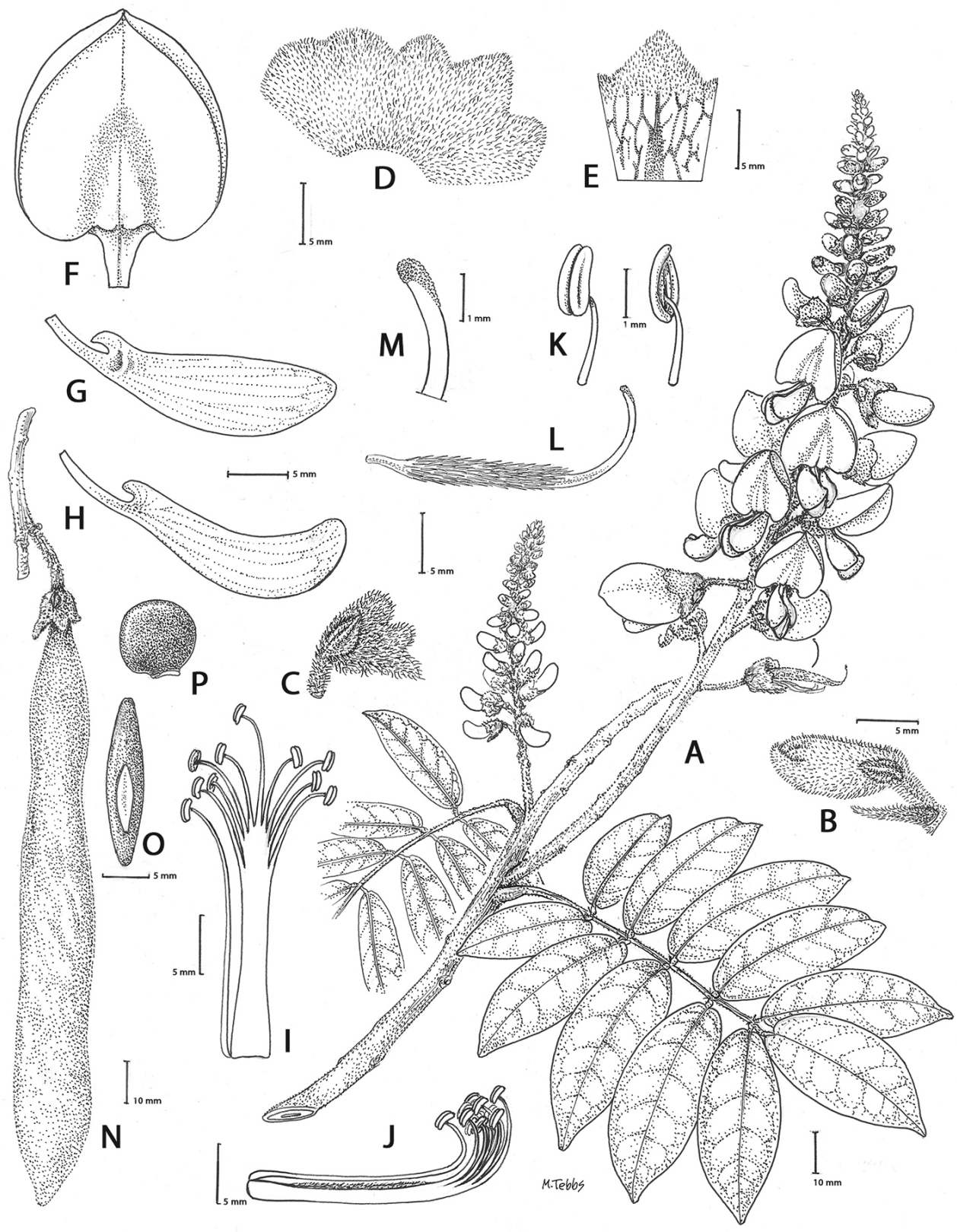

Figure 4. Nanhaia speciosa (Champ. ex Benth.) J.Compton \& Schrire. A Habit B flower bud with bract and bracteole $\mathbf{C}$ calyx exterior and bracteole $\mathbf{D}$ detail of calyx exterior $\mathbf{E}$ detail of calyx interior $\mathbf{F}$ standard petal $\mathbf{G}$ wing petal $\mathbf{H}$ keel petal $\mathbf{I}$ staminal column $\mathbf{J}$ staminal column lateral view $\mathbf{K}$ stamen ventral and dorsal view $\mathbf{L}$ ovary and style $\mathbf{M}$ style and stigma $\mathbf{N}$ pod $\mathbf{O}$ seed lateral view $\mathbf{P}$ seed ventral view (all from Shiu Ling Hu 6091). Drawn by Margaret Tebbs. 
tologue "it is in the Hookerian Herbarium from Millett's collection" and is therefore a syntype in the absence of any cited holotype (Art. 9.6). Under Art. 9.12 a syntype takes precendence over any uncited specimens when selecting a lectotype (Turland et al. 2018). Millett 505 is therefore the obligate lectotype (N. Turland pers. comm.).

Illustrations. Lôc and Vidal in Fl. Cambodge, Laos \& Vietnam 30: 37 (2001); Wei and Pedley Fl. China 10: 182, t. 215 [1-7] (2010). http://www.fpcn.net/a/tengmanzhiwu/20131008/Callerya_speciosa.html (as Callerya speciosa). Fig. 4.

Distribution. China (Fujian, Guangdong, Guangxi, Guizhou, Hainan, Hunan, Yunnan); Vietnam (north).

Habitat. In open forest, edges of ravines and thickets from 100-300 m.

\section{Nanhaia fordii (Dunn) J.Compton \& Schrire, comb. nov.}

urn:lsid:ipni.org:names:77198986-1

三 Millettia fordii Dunn, J. Linn. Soc., Bot. 41: 156 (1912a). Type: China, Guandong,

"Comm. Ford, coll. August 1887, Kwangtung Prov. Lienchow River", [Charles]

Ford 62, K000881044 (K, holo.!); P02141771 (P, iso.!); A00065311 (A, iso!)

三 Callerya fordii (Dunn) Schot, Blumea 39(1-2): 23 (1994)

Illustrations. Lôc and Vidal in Fl. Cambodge, Laos \& Vietnam 30: 36 (2001); Wei and Pedley Fl. China 10: 183, t. 215 [8-12] (2010). http://www.plantphoto.cn (in Home Page enter Callerya fordii)

Distribution. China (Guandong, Guangxi); Vietnam (north).

Habitat. Trailing among rocks and thickets in open sites from sea level to $200 \mathrm{~m}$.

\section{Wisteriopsis J.Compton \& Schrire, gen. nov.} urn:Isid:ipni.org:names:77198975-1

$\equiv$ Millettia Sect. Eurybotryae Dunn, J. Linn. Soc., Bot. 41: 135 (1912a)

Diagnosis. The five species of Wisteriopsis (Fig. 5) possess a fine ring of hairs lining the inner rim of the calyx cup, prominent gibbosities below the stipules and persistent bracts at the base of pedicels (vs. bracts caducous in Callerya s.str.). Wisteriopsis has glabrous standards (vs. sericeous in Callerya s.str.) and wings equalling or slightly shorter than the keel in length (vs. wings much shorter than the keel in Callerya). The unique character of this genus is the staminal column being visible between wings and keel at anthesis, becoming free from the keel after pollinator tripping. See under Nanhaia for comparisons with Wisteriopsis.

Type species. Wisteriopsis japonica (Siebold \& Zucc.) J.Compton \& Schrire $\equiv$ Wisteria japonica Siebold \& Zucc. 
Genus description. Robust twining woody vines attaining 4-18 $\mathrm{m}$ in height climbing over shrubs or sprawling over rocks. Stems greyish brown or brown, terete, young branches finely grey or brown tomentose becoming glabrous (or densely ferrugineous tomentose in $W$. eurybotrya). Leaves deciduous (in $W$. japonica) or evergreen, chartaceous or coriaceous, imparipinnate, rachis 9-20 cm long. Stipules 2-4 $\mathrm{mm}$ long, persistent or caducous, emerging from a subulate or mounded gibbosity. Stipels 1-5 mm long, persistent or caducous, petiolules 3-4 mm. Leaflets (5-) 7-15, in opposite pairs, ovate-lanceolate, elliptic, ovate or ovate-oblong (linear-lanceolate in W. reticulata var. stenophylla) $15-40 \times 5-20 \mathrm{~mm}$, upper surface glabrous with reticulate venation (smooth and shiny in $W$. championii), lower surface paler and glabrous or with hairs along veins, apex obtuse, acute, acuminate or cuspidate, margins entire, base cordate or cuneate. Inflorescence 8-40 cm long, laterally paniculate and sometimes racemose in leaf axils, acropetal, erect or pendulous, sparsely hairy or glabrous. Flowers 7-16 mm long emerging from April to August. Floral bracts persistent, (caducous in W. eurybotrya), linear to subulate, 1-6 mm long, subtending base of pedicel. Bracteoles subtending and adnate to base of calyx, linear, acuminate 1-2 $\mathrm{mm}$ long, persistent. Pedicels elongating at flower maturity, 2-8 $\mathrm{mm}$ long, glabrous or pubescent. Calyx tubular, cupuliform or campanulate, $1.5-4 \times 2-6 \mathrm{~mm}$, glabrous or pubescent externally, with an annulus of fine hairs at the mouth presenting a ciliate margin, five lobed, lobes more or less equal, upper lobes obtuse or deltoid, lower lobes bluntly acute. Standard 6-12 × 4-10 mm, white sometimes flushed pale pink, greenish white, pink or purple, ovate or suborbicular, slightly deflexed backwards near the base, back of standard glabrous, inside with yellow or green nectar guide, callosities of boss type. Wing petals $5-13 \times 2-4 \mathrm{~mm}$, equal in length to the keel petals, each semi-pandurate with a short pair of auricles near the base and a prominent basal claw 1-3 mm long. Wing petals free of the keel at time of anthesis. Keel petals glabrous, united into a semi-pandurate cup, base with conjoined parallel claws, apex obtuse (acute in W. eurybotrya). Stamens diadelphous, nine fused together, the vexillary one free, all curved upwards at apex, staminal column visible between wings and keel at anthesis, becoming free from keel after pollinator tripping (tardily so in W. reticulata). Ovary glabrous, style $2-3 \mathrm{~mm}$ long, curved upwards at apex, stigma punctate. Pods 65-125 × 8-30 mm, linear, ovate or narrowly elliptic, compressed, (inflated in W. eurybotrya), pale green becoming very dark brown, cartilaginous and tardily dehiscent, exocarp glabrous externally (margins thickened in W. eurybotrya), surface finely corrugated, endocarp chartaceous, pale cream, detaching tardily from the exocarp, the seeds in hollow cavities, pods dehiscing explosively, the valves opening straight or twisting to release the seeds, subseptate. Seeds (1-)6-8, lenticular, suborbicular to oblate-spheroidal, smooth, brown, 5-28 × 4-20 × 1-5 mm, hilum 1-2 × $1 \mathrm{~mm}$, elliptic. Fig. 5 .

Distribution. China (Anhui, Fujian, Guizhou, Guangdong, Guangxi, Hainan, Hubei, Hunan, Jiangsu, Jiangxi, Zhejiang); Japan (Honshu, Kyushu, Shikoku); Laos; South Korea (North Gyeongsang, South Gyeongsang, North Jeolla, South Jeolla); Thailand; Vietnam.

Etymology. Wisteria - opsis = like $(\mathrm{Gk})$, resembling the genus Wisteria . 


\section{Key to species of Wisteriopsis}

1 Inflorescences of true terminal panicles without leaves subtending flowering

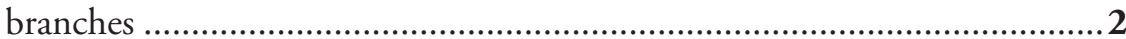

- $\quad$ Inflorescences largely of racemes aggregated terminally on branches, such that the entire flowering region appears as a leafy panicle with only the occasional leaf absent at the base of a flowering branch................................................. 4

2 Inflorescence $20-30 \mathrm{~cm}$ long; apex of wing and keel petals obtuse; pods narrow 5-15 mm wide, not constricted between seeds; seeds 1-6, lenticular, $7-10 \times 6-9 \mathrm{~mm}$. 3

- $\quad$ Inflorescence 30-40 cm long; apex of wing and keel petals acute; pods broad 25-35 mm wide, noticeably constricted between seeds and with thickened dorsal and ventral sutures; seeds 3-6, ellipsoid or suborbicular $15-35 \times 20$ $25 \mathrm{~mm}$

W. eurybotrya

3 Leaflets oblong-ovate, apex acuminate; flowers white, nectar guides green .... W. championii

- $\quad$ Leaflets lanceolate or linear, apex acute; flowers red, pink or purple, nectar guides yellow W. reticulata

4 Leaves deciduous, leaflets 9-15 narrowly ovate-lanceolate; flowers 6-12 mm long

W. japonica

- $\quad$ Leaves evergreen, leaflets 5-7 broadly ovate; flowers $12-15 \mathrm{~mm}$ long W. kiangsiensis

Wisteriopsis japonica (Siebold \& Zucc.) J.Compton \& Schrire, comb. nov. urn:Isid:ipni.org:names:77198989-1

Type. Japan, “Wisteria japonica Fl. Jap. t. 43. Hb. de Siebold 1829”, Siebold s.n. L0059625 (L, lecto.!, designated by Compton and Thijsse (2013)); Japan, "Herb. Lugd. Batav. Coll. Dr von Siebold s.n. donnée par Mr. Blume, Wisteria japonica Zucc.” P02141817 (P, isolecto.!)

Key to the varieties of Wisteriopsis japonica

1 Flowers with all petals cream coloured, pale yellow or greenish-white var. japonica

- $\quad$ Flowers with standard white, wings and keel pink....................var. alborosea

Wisteriopsis japonica var. japonica $\equiv$ Wisteria japonica Siebold \& Zucc., Fl. Jap.: 88. (1839) 

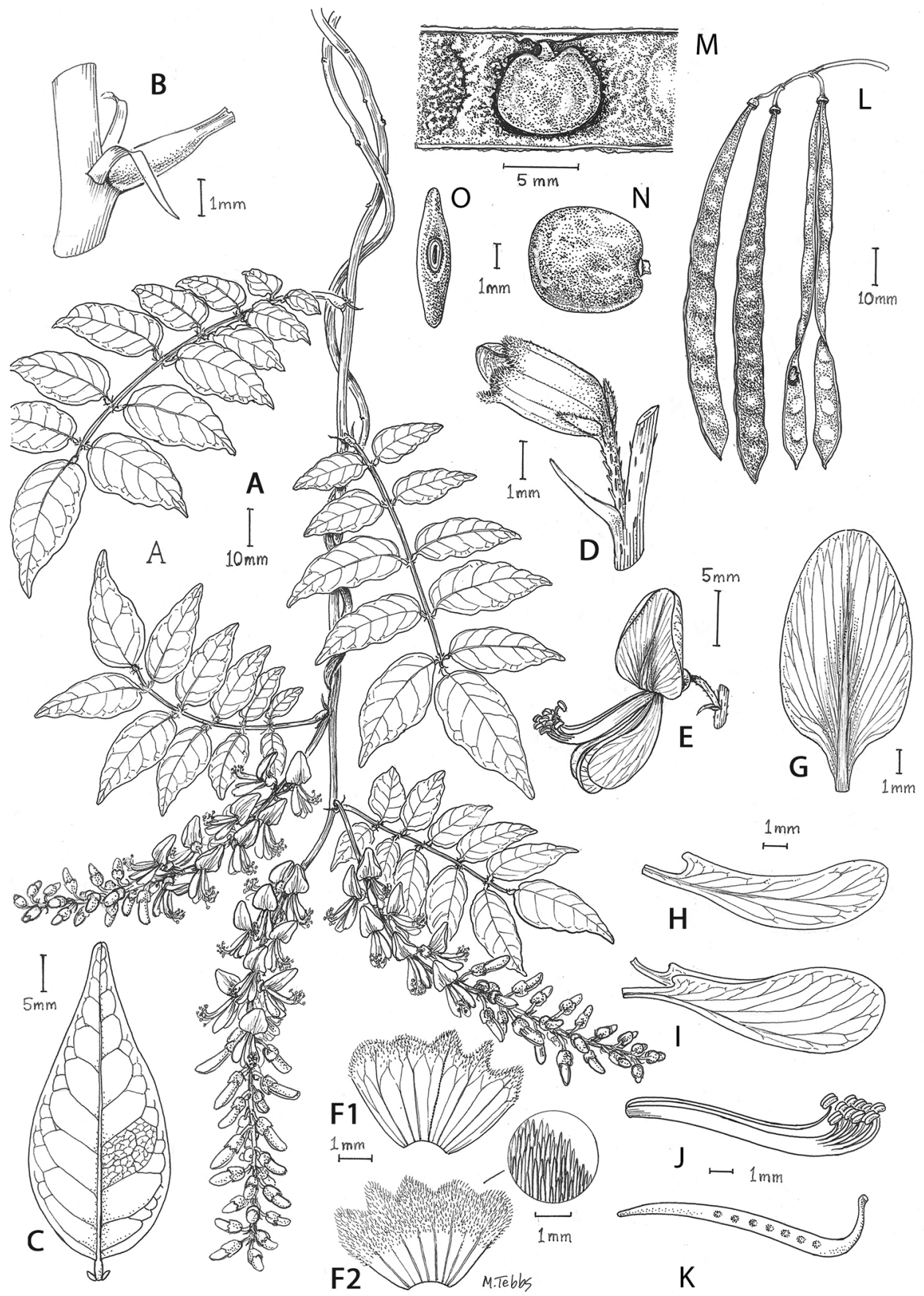

Figure 5. Wisteriopsis japonica (Siebold \& Zucc.) J.Compton \& Schrire. A Habit B stipels C lower surface of leaflet $\mathbf{D}$ flower bud with bract and bracteoles $\mathbf{E}$ flower $\mathbf{F I}$ calyx outer surface $\mathbf{F} \mathbf{2}$ calyx inner surface and detail of hairs $\mathbf{G}$ standard petal inner surface $\mathbf{H}$ wing petal $\mathbf{I}$ keel petal $\mathbf{J}$ staminal column $\mathbf{K}$ ovary and style $\mathbf{L}$ pods $\mathbf{M}$ pod interior and seed $\mathbf{N}$ ventral view of seed $\mathbf{O}$ lateral view of seed $\mathbf{A}-\mathbf{C}, \mathbf{E}-\mathbf{K}$ from Maximowicz s.n. 1863 D from Oldham 386, L-O from Togasi MSM1, 1950. Drawn by Margaret Tebbs. 
E Phaseoloides [Phaseolodes] japonicum (Siebold \& Zucc.) Kuntze, Revis. Gen. Pl. 1: 201. (1891)

$\equiv$ Kraunhia japonica (Siebold \& Zucc.) Taub., Engler \& Prantl, Nat. Pflanzen- fam. 3(3): 271. (1894).

Illustration. Compton in Curtis's Bot. Mag. 32 (3-4): 379, t. 2 (2015); Fig. 5, Plate 3A-D.

Distribution. Japan in south-western Honshu, Shikoku and Kyushu islands; South Korea in North and South Gyeongsang and North and South Jeolla provinces.

Habitat. In woods and forests from sea level to $1200 \mathrm{~m}$, climbing among scrub and trees.

Wisteriopsis japonica var. alborosea (Sakata) J.Compton \& Schrire, comb. nov. urn:Isid:ipni.org:names:77198990-1

三 Millettia japonica (Siebold \& Zucc.) A.Gray f. alborosea Sakata, J. Jap. Bot. 33(1): 30 (1958). Type: Japan, Bansei, Kaseda-shi 18 Aug. 1957, T.Sakata s.n. (KAG, holo.) 三Wisteria japonica Siebold \& Zucc. f. alborosea (Sakata) Yonek., J. Jap. Bot. 80(6): $325(2005)$

Note. The holotype has not been located at KAG where Toshio Sakata's material had been incorporated (Koji Yonekura pers. comm.). No material was available on which to neotypify this taxon.

Distribution. Japan, Kyushu Island.

Wisteriopsis kiangsiensis (Z.Wei) J.Compton \& Schrire, comb. nov. urn:Isid:ipni.org:names:77198991-1

三 Millettia kiangsiensis Z.Wei, Acta Phytotax. Sin. 23(4): 283 (1985). Type: China, Jiangxi Prov., Wuning, Y.G.Xiong 4143 (LBG, holo.); Paratype: China, Anhui, Qin Renchang [Ren-Chang Ching] 2881, elev. 250 m. 30 June 1925, "climber 7 m high", PE00417692 (PE!, K!)

三 Callerya kiangsiensis (Z.Wei) Z.Wei \& Pedley, Flora of China 10: 184, t. 219 [8-15] (2010)

= Millettia kiangsiensis f. purpurea Z.H.Cheng, J. Zhejiang Forest. Coll. 4: 70 (1987). (ZJFC, holo.). China, Zhejiang, Tonglu. This may represent a purple flowered variant of the species but we have not seen any material in order to verify this.

Note. This species described from Jiangxi [originally as Kiangsi] Province in southeastern China, is closely allied to W. japonica. Characters that distinguish W. kiangsiensis from W. japonica are the evergreen leaves in W. kiangsiensis with 7-9 broadly ovate 
leaflets (vs. deciduous leaves with (7 -) 9-15, narrowly ovate-lanceolate leaflets in $W$. japonica). The flowers are white, frequently flushed with pale rose pink and $12-15 \mathrm{~mm}$ long in W. kiangsiensis, (vs. flowers yellowish-white, 6-12 mm long in W. japonica). Both species possess a conspicuous annulus of fine hairs at the mouth of the calyx and narrow lanceolate stipules that arise directly from a deltoid gibbosity positioned on the branch immediately subtending the leaf rachis.

Illustrations. Wei and Pedley, Flora of China 10: 184, t. 219 [8-15] (2010). http://www.plantphoto.cn (In Home Page enter Callerya kiangsiensis).

Distribution. China (Anhui, Fujian, Hubei, Hunan, Jiangsu, Jiangxi, Zhejiang).

Habitat. open sites clambering over scrub among woods and forests up to $500 \mathrm{~m}$.

\section{Wisteriopsis reticulata (Benth.) J.Compton \& Schrire, comb. nov.} urn:Isid:ipni.org:names:77198992-1

Type. China, "woodland hedges, flowers purple, vexillum exauriculatum ecallosum. stam. vexillum liberum, ovarium glabrum, north China comm. Fortune 1845", R.Fortune A95 s.d., K000881030 (K, lecto.! designated here); K000881031 (K, isolecto.!); "Glycine sp. purple, woods and hedges, north of China, August 1844", R. Fortune A95, P02141772 (P, isolecto.!); P02141773 (P, isolecto.!); P02141774 (P, isolecto.!); M0233437 (M, isolecto.!)

Key to varieties of Wisteriopsis reticulata

1 Leaflets ovate-elliptic or oblong, 15-40 mm wide var. reticulata

- Leaflets linear or narrowly lanceolate, 5-12 mm wide var. stenophylla

\section{Wisteriopsis reticulata var. reticulata}

三 Millettia reticulata Benth. Pl. Jungh. [Miquel] 2: 249 (1852)

三 Phaseoloides [Phaseolodes] reticulata (Benth.) Kuntze, Revis. Gen. Pl. 1: 201. (1891)

三 Callerya reticulata (Benth.) Schot, Blumea 39(1-2): 30 (1994).

= Millettia cognata Hance, J. Bot. 18: 260 (1880). Type: China, Hunan “in collibus demissis ad fl. Siang [Xiang] reg. septent. prov. Hunan aest. 1878 Herb. Hance 20708”, T.L.Bullock s.n., BM001217087 (BM, holo.!); A00065365 (A, iso.!)

三 Phaseoloides [Phaseolodes] cognata (Hance) Kuntze, Revis. Gen. Pl. 1: 201. (1891). = Millettia purpurea Yatabe, Bot. Mag. (Tokyo) 6: 379 (1892). Type: Japan [Icon] Bot. Mag. (Tokyo) 6: t. 12 (1892), (lecto.!, designated here).

Illustrations. Lôc and Vidal in Fl. Cambodge, Laos \& Vietnam 30: 32 (2001); Wei and Pedley Fl. China 10: 183, t. 216 [7-14] (2010). https://singapore.biodiversity. 
online (Callerya reticulata) https://www.flickr.com/photos/reulim/34692959202; https://florafaunaweb.nparks.gov.sg/special-pages/plant-detail.aspx?id=3329 (Plate 3E).

Distribution. China (Anhui, Fujian, Guangdong, Guangxi, Guizhou, Hainan, Hubei, Hunan, Jiangsu, Jiangxi, southern Shaanxi, Sichuan, Taiwan, Yunnan and Zhejiang); Vietnam north.

Habitat. On open slopes covering wooded thickets from sea level to $1000 \mathrm{~m}$.

Wisteriopsis reticulata var. stenophylla (Merr. \& Chun) J.Compton \& Schrire, comb. nov.

urn:lsid:ipni.org:names:77198993-1

三 Millettia reticulata var. stenophylla Merr. \& Chun, Sunyatsenia 5: 83 (1940). Type: China, Hainan, Yaichow [Yazhou], "29 May 1933, Longzhou, Licai, alt. 800 $\mathrm{ft}$. vine on rocks twining on shrubs, fls purplish-red fragrant", F.C.How 70826, IBSC000909 (IBSC, holo.); US02324730 (US, iso.!); P02754288 (P, iso.!); (SYS, iso.); NAS00391016 (NAS, iso.!); IBK00073921 (IBK, iso.!). Paratype: China, Hainan, Po-ting, [Baoting]. F.C.How 73744 "elev. $1200 \mathrm{ft}$. along streams, twining, 26 September 1935” A00063957 (A!)

三 Callerya reticulata var. stenophylla (Merr. \& Chun) X.Y.Zhu, Legumes of China (ILDIS) 455 (2007)

Illustration. Wei and Pedley Fl. China 10: 184, t. 216 [15] (2010).

Distribution. China, Hainan Island.

Habitat. Open sites in tropical forest.

Wisteriopsis championii (Benth.) J.Compton \& Schrire, comb. nov. urn:lsid:ipni.org:names:77198994-1

三 Millettia championii Benth., Hooker's Journ. Bot. Kew Gard. Misc. 4: 74 (1852).

Type: China, Guangdong, Hong Kong, [John George] Champion 263, lower specimen K000881035 (K, lecto.! designated here); China, Guangdong, Hong Kong, Champion 263, upper specimen K000881036 (K, isolecto.!)

三 Phaseoloides [Phaseolodes] championii (Benth.) Kuntze, Revis. Gen. Pl. 1: 201. (1891) 三 Millettia reticulata var. championii (Benth.) H.Sun, Fl. Yunnanica 10: 404 (2006) 三 Callerya championii (Benth.) X.Y.Zhu, Legumes of China (ILDIS) 450 (2007)

Illustration. Wei and Pedley Fl. China 10: 184, t. 219 [1-7] (2010). http://www.hkwildlife.net/Forum/viewthread.php?tid=4986\&page=1 (as Millettia championii).

Distribution. China (Fujian, Guandong, Guangxi, Hong Kong and Jiangxi).

Habitat. In thickets beside rocky valleys climbing among rocks and scrub from 200 to $800 \mathrm{~m}$. 
Wisteriopsis eurybotrya (Drake) J.Compton \& Schrire, comb. nov. urn:Isid:ipni.org:names:77198995-1

E Millettia eurybotrya Drake, J. Bot. (Morot) 1891: 187 (1891). Type: Vietnam,

"Tonkin, Thu Phap [Van Hoa, Hanoi], liane, corolle rose, 1887", [Gaspard Joseph Benedict] Balansa 2304, P02141769 (P, lecto.!, designated by Schot (1994): P02141770 (P, isolecto.!); K000881012 (K, isolecto.!). Paratypes: B.Balansa 2303, “Tu-Phap dans les bois, August 1887” P02753447 (P!); B.Balansa 2303 P00852397 (P, isopara!); B.Balansa 2300, "Tu-Phap dans les bois, September 1887 ” P02753453 (P!); B.Balansa 2300 P027533454 (P!); P02753446; B.Balansa 2301, “Tonkin, Tho-bo dans les forets, November 1887” (P!) 三 Callerya eurybotrya (Drake) Schot, Blumea 39(1-2): 22 (1994)

Illustrations. Lôc and Vidal in Fl. Cambodge, Laos \& Vietnam 30: 30, t. 7 (2001); Wei and Pedley Fl. China 10: 182, t. 216 [1-6] (2010). http://www.plantphoto.cn (as Callerya eurybotrya).

Distribution. China (Guangdong, Guizhou); Thailand; Vietnam.

Habitat. In thickets, river margins and on the edge of evergreen forests from sea level to $400 \mathrm{~m}$.

Clade C - Callerya, Serawaia, Whitfordiodendron, Kanburia and Afgekia Fig. 1; Supp. material 1: Figs S1-S6

\section{Callerya Endl., Gen. Pl. Suppl. 3: 104 (1843), emend nov. J.Compton and Schrire}

$\equiv$ Millettia Sect. Curvistylae Z.Wei, Acta Phytotax. Sin. 23(4): 284 (1985)

= Millettia Ser. Dielsianae Z.Wei, Acta Phytotax. Sin. 23(4): 284 (1985) Type species: Millettia dielsiana Harms ex Diels, Bot. Jahrb. Syst. 29(3-4): 412 (1900).

= Millettia Ser. Oospermae Z.Wei, Acta Phytotax. Sin. 23(4): 284 (1985) Type species: Millettia oosperma Dunn, J. Linn. Soc. Bot. 41: 157 (1912a).

Diagnosis. Callerya s.str. is here recognised as comprising C. nitida, C. bonatiana, $C$. cochinchinensis, C. cinerea and C. dielsiana. The Flora of China account (Wei and Pedley 2010), included a further eight species: C. tsui, C. dorwardii, C. sphaerosperma, $C$. congestiflora, C. longipedunculata, C. gentiliana, C. oosperma and C. sericosema which we have not been able to ascertain the status of due to a paucity of study material. Their descriptions in Wei and Pedley (2010), however, indicate that they should be placed in Callerya s.str. The flowers in Callerya s.str. have wing petals shorter than the keel petals (vs. equal or longer in Kanburia and Whitfordiodendron). The standard is also proportionately larger than in Kanburia and Whitfordiodendron. The keel is glabrous in Callerya s.str. (vs. sericeous in Whitfordiodendron). 
Type species. Callerya nitida (Benth.) R.Geesink $\equiv$ Millettia nitida Benth.

Genus description. Short scandent vines scrambling over rocks or shrubs to $0.5-1$ $\mathrm{m}$ tall, or tall scrambling climbers to $20 \mathrm{~m}$ tall. Stems grey, yellowish or brown, terete, pubescent or glabrescent. Leaves with 3-13 leaflets, evergreen, glabrous or strigose, (villose in C. bonatiana) imparipinnate, rachis 3-16 (-40) cm long. Stipules $1-4 \mathrm{~mm}$ long, deltoid, caducous (persistent on C. nitida). Stipels 2-7 mm long, linear, persistent (absent in C. bonatiana). Leaflets 3-15 $(-22)$ x 2-6 $(-10) \mathrm{cm}$, terminal leaflet distinctly larger than laterals, basal pair usually smallest; lateral leaflets, ovate or obovate or lanceolate, ovate-elliptic or narrowly elliptic, glabrous or pubescent (densely villose below in C. bonatiana), apex obtuse or acute or acuminate, margins entire, base rounded, cuneate or subcordate. Inflorescence a terminal panicle 6-20 $(-40) \mathrm{cm}$ long, (racemes axillary $8-12 \mathrm{~cm}$ long in a leafy panicle in C. bonatiana), peduncle yellow or brown puberulous or tomentose. Flowers 11-25 mm long, emerging from March - November. Floral bracts 1-6 mm long, narrowly ovate, deltoid or linear, caducous. Bracteoles at top of pedicel 1-6 mm long, (reflexed in C. nitida) narrowly ovate, deltoid or linear, caducous or persistent. Pedicels $2-10 \mathrm{~mm}$ long, tomentose or puberulent. Calyx 3-12 $\times 4-10 \mathrm{~mm}$ broadly campanulate, oblique, sparsely pubescent or densely sericeous externally, five lobed, teeth unequal 1-6 mm long, obtuse, subtruncate or acute. Standard 12-25 × 8-17 mm, elliptic or ovate, white, pink, lilac, red, mauve, violet, green or purple, nectar guide yellow or green, back of standard densely white, yellow or brown sericeous, apex acute, retuse or obtuse (nectar guide fringed with hairs on inner face on C. bonatiana), callosities of ridge or boss type. Wing petals $5-15 \times 2-5 \mathrm{~mm}$, glabrous, shorter than the keel, each narrowly obovate, straight at apex; free from the keel, apex obtuse, basal claws 2-5 mm long. Keel petals 8-16 × 3-6 mm, glabrous, united into a falcate, navicular cup, apex obtuse. Stamens diadelphous, nine fused together, the vexillary one free, all curved upwards at apex. Ovary densely sericeous, tomentose or velutinous, style 6-9 mm long, ciliate at base (C. cochinchinensis) or glabrous, curved upwards at apex, stigma punctate. Pods 4-15 × 1.5-4 cm, flat or inflated, linear, linear-oblong, rhomboid-oblong rarely globose, straight or torulose, dehiscent, surface grey, brown or yellow tomentose, subseptate. Seeds (1 -) 2-5, ovoid, orbicular, oblately-spheroid or ellipsoid, 8-30 × 6-35 × 2-20 mm, hilum central, elliptic or oval 2-5 × 0.5-1 mm.

Distribution. Bangladesh; Bhutan; China (Anhui, Fujian, Guandong, Guangxi, Guizhou, Hainan, Hubei, Hunan, Jiangxi, Sichuan, Shaanxi, Yunnan, Zhejiang); India; Laos; Myanmar; Nepal; Thailand; Vietnam.

Etymology. The genus Callerya is named after Joseph Gaetan Pierre-Maxime-Marie Callery (1810-1862) scholar, missionary and sinologist.

\section{Key to species of Callerya recognized in this treatment}

$1 \quad$ Leaves 11-13 foliolate; standard bright green, nectary guide surrounded by sericeous hairs on inner face; wing petals lilac-purple; style glabrous....C. bonatiana

- $\quad$ Leaves 3-5 foliolate; standard glabrous within 
2 Leaves 3(-5) foliolate; standard white sometimes flushed green, style ciliate or sericeous at base; seeds $1-2$

C. cochinchinensis

- $\quad$ Leaves 5-foliolate; standard pink, lilac, red or purple, style glabrous; seeds $(1-) 3-5$ 3

3 Stipels subulate, 1-2 mm long; bracteoles reflexed; pods linear-oblong, not inflated C. nitida

- $\quad$ Stipels linear 3-4 mm long; bracteoles straight; pods inflated .....................4

4 Floral bracts lanceolate 3-5 mm long; seeds $3-5$ per pod, $8 \times 6 \mathrm{~mm}$, tawny brown, oblong or suborbicular. C. dielsiana

- $\quad$ Floral bracts narrowly ovate to linear 2-6 mm long; seeds (1 -)2-4 per pod, $18 \times 14 \mathrm{~mm}$, dark violet, ellipsoid C. cinerea

\section{Callerya nitida (Benth.) R.Geesink, Leiden Bot. Ser. 8: 83 (1984)}

Type. "Hong Kong, China, received by W. J. Hooker 1841", Mr Millett [Charles] s.n., K000881042 (K, lecto.! designated here); K000881039 (K, isolecto.!); K000881043 (K, isolecto.!)

\section{Key to varieties of Callerya nitida}

1 Flowers 22-24 mm long; leaflets 50-90 (-110) × 30-40 mm., surfaces glabrous or sparsely pubescent below, apex acute. var. nitida

- $\quad$ Flowers 16-18 mm long; leaflets 35-55 × 20-30 mm .............................. 2

2 Leaflets narrowly lanceolate, both surfaces glabrous, apex acuminate

var. minor

- Leaflets ovate, densely reddish-brown hirsute below, rough but glabrous above, apex cuspidate. var. hirsutissima

\section{Callerya nitida var. nitida}

三 Millettia nitida Benth., London J. Bot. 1: 484 (1842)

三Phaseoloides [Phaseolodes] nitida (Benth.) Kuntze, Revis. Gen Pl. 1: 201 (1891)

= Millettia kueichouensis Hu, Acta Phytotax. Sin. 3: 356 (1954). Type: China, Guizhou, Fengxiangxi, 10 June 1928, P.Q. [Pu Chiu] Tsoong 749, PE00022411 (PE, holo.!).

Illustrations. Schot, Blumea 39(1-2): 27, t. 3 (1994); Wei and Pedley Fl. China 10: 182, t. 221 [1-8] (2010). http://lalajacky.blogspot.com/2008/11/blog-post_5886. html (as Millettia nitida).

Distribution. China (Fujian, Guangdong, Guangxi, Guizhou, Hainan, Hunan, Jiangxi, Sichuan, Taiwan, Yunnan, Zhejiang).

Habitat. In thickets and lowland forest margins from sea level to $1500 \mathrm{~m}$. 


\section{Callerya nitida var. minor (Z.Wei) X.Y.Zhu, Legumes China 454 (2007)}

三 Millettia nitida var. minor Z.Wei, Acta Phytotax. Sin. 23: 288 (1985). Type: China, Sichuan, Mt. Emei elev. 900 m., Lushan county, Bailongdong, Houshan, 4 September 1957, G.H.[Guang Hui] Yang 57095, PE01432522 (PE, holo.!)

Illustration. Wei and Pedley Fl. China 10: 182, t. 221 [9] (2010).

Distribution. China (Fujian, Guangdong, Guangxi, Hunan, Jiangxi).

Habitat. in thickets and along forest margins and open places 500 to $1000 \mathrm{~m}$.

\section{Callerya nitida var. hirsutissima (Z.Wei) X.Y.Zhu, Legumes China 454 (2007)}

三 Millettia nitida var. hirsutissima Z.Wei, Acta Phytotax. Sin. 23: 288 (1985). Type: China, Hunan, Zixing, B.H. [Pao Han] Liang 85983 (IBSC, holo.)

Illustration. Wei and Pedley Fl. China 10: 182, t. 221 [10] (2010).

Distribution. China (Fujian, Guangdong, Guangxi, Guizhou, Hunan, Jiangxi, Sichuan, Yunnan, Zhejiang).

Habitat. in thickets and along forest margins 800 to $1500 \mathrm{~m}$.

Callerya dielsiana (Harms ex Diels) P.K.Lôc ex Z.Wei \& Pedley, Fl. China 10: 187, t. $222[1-7] 2010$

三 Millettia dielsiana Harms ex Diels, Bot. Jahrb. Syst. 29(3-4): 412 (1901). Type: China "Setchuen [Sichuan] ab incolis collectae" fl. Nanchuan, s.d. 1899, [Carl] Bock \& [Augustus] von Rosthorn 1626, V-2014532 (O, lecto.! designated here); A00339036 (A, isolecto.!) Paratype: China, Sichuan, Nanchuan s.d., Bock \& Rosthorn 1638, V-2014531 (O!)

Distribution. China (Anhui, Fujian, Gansu, Guangdong, Guangxi, Guizhou, Hainan, Hubei, Hunan, Jiangxi, Shaanxi, Sichuan, Yunnan, Zhejiang).

Habitat. In open places, mixed woods and forest margins from 300 to $2500 \mathrm{~m}$.

Callerya bonatiana (Pamp.) P.K.Lôc, Bot. Zhurn. (Moscow \& Leningrad) 81(10): 99 (1996)

三 Millettia bonatiana Pamp., Nuovo Giorn. Bot. Ital. 17: 24 (1910). Type: China, Yunnan "pagoda de Ke-long, 23 Mai 1904, plante grimpante" [François] Ducloux 380, FI-1018361 (FI, lecto.! designated here); FI-1018360 (FI, isolecto.!); Paratypes: China, Yunnan, "Juin 1904, fleurs vertes, grimpes au sommet des arbres", [Edouard-Ernest] Maire 156, FI-1018363 (FI!); P03583784 (P, isopara.!); China, 
Yunnan, [E.E] Maire 196, "Octobre 1904, fruits de la grande legumineuse a fleurs vertes” FI-1018362 (FI!); P02141757 (P, isopara.!); P03583784 (P, isopara.!)

Illustrations. Wei and Pedley Fl. China 10: 183, t. 210 [6-13] (2010) http://www. plantphoto.cn (in Home Page enter Callerya bonatiana).

Distribution. China (Guangxi, Yunnan); Vietnam.

Habitat. tall climber among trees and over shrubs from 200-1000 m.

\section{Callerya cochinchinensis (Gagnep.) Schot, Blumea 39(1-2): 19 (1994)}

三 Millettia cochinchinensis Gagnep., Notul. Syst. (Paris) 2: 353 (1913). Type: Vietnam, Dong Nai, "Cochinchine, vers Pho-qua, dans la Prov. de Bien-hoa, Mars 1877”, [Jean-Baptiste Louis] Pierre s.n., P02141765 (P, holo.!); P02141766 (P, iso.!); P02141767 (P, iso.!); BM000997331 (BM, iso.!); K000881015 (K, iso.!)

Illustration. Lôc and Vidal in Fl. Cambodge, Laos \& Vietnam 30: 38, t. 8 (2001).

Distribution. Vietnam (south).

Habitat. In light scrub and open places along riverbanks, forest margins and ravines from 300 to $1000 \mathrm{~m}$.

\section{Callerya cinerea (Benth.) Schot, Blumea 39(1-2): 17, t. 2 (1994)}

EMillettia cinerea Benth. Pl. Jungh. [Miquel] 2: 249 (1852). Type: Bangladesh, “Pongamia cinerea, Sillet TD”, Wallich Cat. 5888A, K000881025 (K, holo.!); Paratypes: Wallich Cat. 5888B, "Pongamia cinerea, Chittagong HB" K000881022 (K!); K000881024 (K, para.!); BM000997333 (BM!); BM000997334 (BM!) 三 Phaseoloides [Phaseolodes] cinerea (Benth.) Kuntze, Revis. Gen Pl. 1: 201 (1891) = Millettia bracteosa Gagnep., Not. Syst. 2(11):352 (1913). Type: China, Yunnan, "bois vers Tchen-fong-shan [Feng shan], August 1894", [Jean, Marie] Delavay s.n., P02141760 (P, lecto.! designated here); P02141761 (P, isolecto.!); P02141762 (P, isolecto.!). $=$ Millettia heterocarpa Chun ex T.C.Chen, Acta Phytotax. Sin. 3:364 (1955). Type: China, Guangdong, Heitan Jiao, 8 August 1930, Nian Qi Chen 41503, IBSC000908 (IBSC, holo.) $\equiv$ Millettia dielsiana var. heterocarpa (Chun ex T.C.Chen) Z.Wei, Acta Phytotax. Sin. 23(4): 289 (1985) E Callerya dielsiana var. heterocarpa (Chun ex T.C.Chen) X.Y.Zhu ex Z.Wei \& Pedley, Fl. China 10: 187 (2010).

= Millettia dielsiana var. solida T.C.Chen ex Z.Wei, Acta Phytotax. Sin 23(4): 289 (1985). Type: China, Hunan Qianyang, Z.T.Li 3130 (IBSC, holo.) E Callerya dielsiana var. solida (T.C.Chen ex Z.Wei) X.Y.Zhu ex Z.Wei \& Pedley, Fl. China 10: 187 (2010).

Illustrations. Lôc and Vidal in Fl. Cambodge, Laos \& Vietnam 30: 43 (2001); Lewis in Curtis's Bot. Mag. n.s. 29 (2): 141, Pl. 732 (2012); Wei and Pedley Fl. China 10: 
184, t. 217 [1-2] (2010). http://www.plantthis.com.au/plant-information.asp?garden er $=27142 \&$ tabview=photos\&plantSpot=

Distribution. Bangladesh; Bhutan; China (Jiangxi, Fujian, Guangdong, Guangxi, Guizhou, Hunan, Sichuan, Xizang, Yunnan); India; Myanmar; Nepal; Thailand.

Habitat. In broad-leaved forest margins, ravines, streamsides and thickets from 150 to $1200 \mathrm{~m}$.

\section{Serawaia J.Compton \& Schrire, gen. nov.} urn:Isid:ipni.org:names:77198976-1

Diagnosis. This monospecific genus has several autapomorphies compared with other genera within the tribe. It is the only species that has large and very persistent imbricate floral bracts along the inflorescence enclosing the uniquely golden-yellow flowers. Serawaia is the only genus in Clade D that has prominent gibbosities below the stipules. Its nearest affinities lie with Callerya, Kanburia and Whitfordiodendron which all have sericeous backs to their standard petals. The back of the standards of Serawaia are, however, pubescent but the hairs are not as long as those in Afgekia, the other member of Clade D. The wing petals, which are free from the keel, almost equal the length of the keel as in Kanburia, Whitfordiodendron and Afgekia which distinguishes these four genera readily from Callerya whose wings are shorter. The ovary in Serawaia is only sparsely hairy whereas in all four other genera within Clade D the ovaries are densely sericeous (see figs 4 and 5 in Schot (1994: 33, 34).

Type species. Serawaia strobilifera (Schot) J.Compton \& Schrire $\equiv$ Callerya strobilifera Schot.

Genus description. Scandent twining vines scrambling up trees and along river banks to $8 \mathrm{~m}$ high. Stems very pale grey or white, terete, glabrous. Leaves with 5-7 leaflets, evergreen, glabrous, imparipinnate, rachis $7-20 \mathrm{~cm}$ long. Stipules $5-8 \mathrm{~mm}$ long, linear, persistent, arising from above prominent gibbosities. Stipels 3-4 mm long, linear, persistent. Leaflets $4-14 \times 2-7 \mathrm{~cm}$, broadly or narrowly elliptic, glabrous on both surfaces, apex acuminate or cuspidate, margins entire, base rounded to subcordate. Inflorescence erect, sometimes leafy few-branched panicles $12-20 \mathrm{~cm}$ long, peduncle pale grey, glabrous. Flowers 15-21 mm long, emerging from May to August. Floral bracts 15-18 $\times 8-12 \mathrm{~mm}$, persistent, with longitudinal parallel venation, overlapping flower buds in a strobilate inflorescence. Bracteoles 6-7 mm long, at top of pedicel, linear, persistent. Pedicels 4-6 mm long, glabrous. Calyx 3-6 × 4-6 mm campanulate, oblique, pubescent externally, five lobed, teeth distinctly unequal 2-6 mm long, acute, ciliate. Standard 15$18 \times 11-17 \mathrm{~mm}$, suborbicular, bright lemon or golden yellow, nectar guide yellow, back of standard sparsely pubescent, apex retuse, callosities of boss type. Wing petals 12-14 × 4-5 mm, glabrous, subequal to the keel, each semi-pandurate, slightly curved upwards at the apex; completely free from the keel, apex obtuse, basal claw 2-3 mm long. Keel petals 11-13 × 4-5 mm, glabrous, united into a falcate, navicular cup, apex obtuse, basal claw 3-4 mm long. Stamens diadelphous, nine fused together, the vexillary one free, all curved upwards at apex. Ovary sparsely pubescent, style glabrous, 2-3 mm long 
curved upwards at apex, stigma punctate. Pods 19-30 × 2-2.5 cm, flat, linear, or obovate, dehiscent, surface shortly hirsute, smooth, brown and hard when dry, subseptate. Seeds 2-3, flattened-orbicular, $17 \times 17 \times 10 \mathrm{~mm}$, hilum central, elliptic 2-3 $1 \mathrm{~mm}$.

Etymology. named after the Serawai river in west Kalimantan, a tributary of the Kapuas river, where the species was first discovered.

\section{Serawaia strobilifera (Schot) J.Compton \& Schrire, comb. nov.} urn:1sid:ipni.org:names:77198996-1

三 Callerya strobilifera Schot, Blumea 39(1-2): 32, t. 4 \& 5 (1994). Type: Indonesia, Kalimantan, "Borneo west, Serawai distr., Lebang Hara”, 24 November 1924, Hans Winckler 350, L0018804 (L, holo.!); E00301097 (E, iso.!)

Illustrations. Schot in Blumea 39(1-2): 33 fig. 4; 34 fig. 5 (1994) (as Callerya strobilifera). Distribution. Borneo. Indonesia: Kalimantan, (central and east); Malaysia (Sabah). Habitat. In open sites climbing among trees and scrub on exposed ridges and riverbanks from sea level to $350 \mathrm{~m}$.

\section{Whitfordiodendron Elmer, Leafl. Philipp. Bot. 2: 689, 743 (1910), emend nov. J.Compton \& Schrire}

Diagnosis. The four species of Whitfordiodendron share several characters with the new genus Kanburia but bracteoles are present on the calyx and persistent in Whitfordiodendron (vs. absent in Kanburia). The keel petals are densely sericeous in Whitfordiodendron (vs. glabrous in Kanburia and Callerya). The pods in Whitfordiodendron are inflated and ovoid with a velutinous or pubescent surface (vs. linear, compressed, glabrescent in Kanburia). The ovoid seeds in Whitfordiodendron may become fused together when there are more than one per pod (vs. lenticular, separate in pod in Kanburia). The wing petals are equal in length with the keel petals in Whitfordiodendron (vs. shorter in Callerya).

Type species. Whitfordiodendron scandens Elmer.

Genus description. Scrambling climbers 10-20 (- 40) m tall. Stems grey or brown, terete, glabrous or finely grey puberulent. Leaves with 3-13 leaflets, evergreen, nitid above, glabrous or sparsely pubescent, imparipinnate, rachis $9-25 \mathrm{~cm}$ long. Stipules 1-4 mm long, narrowly deltoid, caducous (persistent W. erianthum). Stipels absent. Leaflets large, 4-15 (-25) x 2-9 (-12) cm, ovate, narrowly elliptic or obovate, apex acuminate to cuspidate, margins entire, base rounded or obtuse or acute. Inflorescence a terminal panicle 5-20 cm long, peduncle sericeous (cauliflorous and glabrescent $20-60 \mathrm{~cm}$ long in $W$. nieuwenhuisii). Flowers $8-23 \mathrm{~mm}$ long, emerging from February - November (May to January W. nieuwenhuisii). Floral bracts 2-7 mm long, ovate, obovate or elliptic, caducous. Bracteoles at base of or on 
the calyx 2-7 mm long, obovate, acute or acuminate, persistent. Pedicels $0.5-2 \mathrm{~mm}$ long, pubescent or sericeous. Calyx 2-9 × 3-5 mm campanulate, oblique, ferrugineous, golden or silvery pubescent or sericeous externally, five lobed, teeth unequal $(0.5-)$ 2-4 mm long, acuminate, pubescent on teeth. Standard 8-18 $\times 9-16 \mathrm{~mm}$, suborbicular or elliptic, inner surface greyish pink, white flushed purple, red, maroon or claret, nectar guide yellow or green, back of standard densely red or golden-brown sericeous, apex acute or obtuse. Callosities ridge or boss type. Wing petals $8-18 \times$ 2-5 mm, sparsely pubescent or ciliate along lower margin (sericeous at apex in $W$. erianthum), equal in length to the keel, broadly obovate, free from the keel, apex obtuse, basal claws 2-4 mm long. Keel petals $8-10 \times 3-5 \mathrm{~mm}$, sericeous externally especially along lower margin, obovate, claw 2-4 mm long, apex obtuse. Stamens diadelphous, nine fused together, the vexillary one free, all curved upwards at apex. Ovary sericeous, style $2-4 \mathrm{~mm}$ long, ciliate, curved upwards at apex, stigma punctate. Pods 4-10 × 2-5 cm, inflated, ovate or obovate, with two thickened margins either side of suture on both sides of pod, dehiscent, surface rugose or ruminate or sparsely pubescent or pale brown velutinous, subseptate. Seeds $1-3$, broadly ellipsoid or ovoid, $12-45 \times 14-35 \times 8-30 \mathrm{~mm}$ (often fused together when more than one), hilum central, broadly elliptic 3-5 × 1-2 mm.

Distribution. Brunei; Indonesia (Sumatra, Borneo: west Kalimantan); Malaysia (Peninsula, Borneo: Sabah, Sarawak); Philippines.

Etymology. Whitfordiodendron for Whitford and dendron = tree (Gk). The genus commemorates Harry Nichols Whitford (1872-1941) world authority on the economics of rubber and on the native forests of the Philippines.

\section{Key to species of Whitfordiodendron}

1 Panicles emerging directly from the main trunk (cauliflorous)

W. nieuwenhuisii

- Panicles terminal on branches .............................................................. 2

2 Flowers 20-23 mm long; calyx 8-9 mm long; stipules persistent

W. erianthum

- $\quad$ Flowers 11-15 mm long; calyx 3-4 mm long; stipules caducous 3

3 Flowers 11-13 mm long; stipules 3-4 $\mathrm{mm}$ long W. sumatranum

- $\quad$ Flowers 13-15 mm long; stipules 1-2 $\mathrm{mm}$ W. scandens

\section{Whitfordiodendron scandens Elmer, Leafl. Philipp. Bot. 2: 689-691, 743 (1910)}

Type. Philippines, Sibuyan Island, Capiz Province [Romblon], Magellanes [Magdiwang] Mt. Giting-Giting [Guiting-Guiting] April 1910, [Adolph Daniel Edward] Elmer 12259, K000880985 (K, lecto.! designated here; holotype PNH destroyed see note below); A00063379 (A, isolecto.!); BM000997328 (BM, isolecto.!); BO- 
1246846 (BO, isolecto.!); E00683153 (E, isolecto.!); E00683253 (E, isolecto!); GH00052081 (GH, isolecto!); MO-022334 (MO, isolecto.!); U0226578 (U, isolecto.!); US00003630 (US, isolecto.!); P03347973 (P, isolecto.!) $\equiv$ Adinobotrys scandens (Elmer) Dunn, Bull. Misc. Inform. Kew 1912(8): 365 (1912) 三 Callerya scandens (Elmer) Schot, Blumea 39(1-2): 31 (1994).

Note. The holotype deposited by Elmer in PNH was destroyed by fire during World War II (T. Circle pers. comm.).

Illustrations. http://www.phytoimages.siu.edu/imgs/pelserpb/r/Fabaceae_Callerya_scandens_43453.html

Distribution. Philippine Islands (Mindanao, Palawan, Panay, Sibuyan).

Habitat. Climbing among lowland forest margins and in thickets from sea level to $200 \mathrm{~m}$.

Whitfordiodendron nieuwenhuisii (J.J.Sm.) Dunn, Bull. Misc. Inform. Kew 1912(8): 364 (1912b)

三 Millettia nieuwenhuisii J.J.Sm., Bull. Dépt. Agric. Indes Néerl. 3: 17 (1906). Type: Indonesia, Kalimantan, [Borneo], Bloe-oe [Bluu river], [cult.] Buitenzorg, Java, 1897-1898, [Anton Willem] Nieuwenhuis 1294 (BO, holo.)

三 Adinobotrys nieuwenhuisii (J.J.Sm.) Dunn, Bull. Misc. Inform. Kew 1911(4): 196

(1911) E Callerya nieuwenhuisii (J.J.Sm.) Schot, Blumea 39(1-2): 26 (1994)

= Adinobotrys myrianthus Dunn, Bull. Misc. Inform. Kew 1911(4): 196 (1911). Type:

"Sarawak, 1865-1868", [Odoardo] Beccari 875, K000881001 (K, lecto.! designated here); S08-15160 (S, isolecto.); P03659572 (P, isolecto.!); M-0233444 (M, isolecto.!) $\equiv$ Whitfordiodendron myrianthum (Dunn) Dunn, Bull. Misc. Inform. Kew 1912(8): 364 (1912).

= Millettia cuspidata Ridl., Bull. Misc. Inform. Kew 1929(8): 254 (1929). Type:

"Sarawak, Matang woods, flowers pinkish", January 1915, [Henry Nicholas] Ridley s.n., K000880995 (K, holo.!).

Illustration. http://www.asianplant.net/Fabaceae/Callerya_nieuwenhuisii.htm

Distribution. Brunei; Indonesia (Borneo: Kalimantan); Malaysia (Borneo: Sarawak, Sabah).

Habitat. Climbing near rivers or on steep slopes in evergreen forest from sea level to $1300 \mathrm{~m}$.

Whitfordiodendron erianthum (Benth.) Dunn, Bull. Misc. Inform. Kew 1912(8): 364 (1912b)

三 Millettia eriantha Benth. Pl. Jungh. [Miquel] 2: 250 (1852). Type: "Malacca, Griffith" Herb. East India Company, 1836, received RBG Kew 1861-1862, Herb. [William] Griffith, K000881008 (K, lecto.! designated by Schot (1994)); 
K000881006 (K, isolecto.!); K000881007 (K, isolecto.!); BM000997329 (BM, isolecto.!); P02753485 (P, isolecto.!)

三Phaseoloides [Phaseolodes] eriantha (Benth.) Kuntze, Revis. Gen Pl. 1: 201 (1891)

三Adinobotrys erianthus (Benth.) Dunn, Bull. Misc. Inform. Kew 1911(4): 196 (1911)

三 Padbruggea eriantha (Benth.) Craib, Fl. Siam. 1: 397 (1928)

三 Callerya eriantha (Benth.) Schot, Blumea 39(1-2): 21 (1994)

Illustration. https://singapore.biodiversity.online (in Home Page enter Callerya eriantha).

Distribution. Brunei; Indonesia (Borneo: Kalimantan and Sumatra); Malaysia (Peninsula, Borneo: Sabah, Sarawak).

Habitat. In rain forest and along wooded cliffs from sea level to $600 \mathrm{~m}$.

Whitfordiodendron sumatranum Merr., Pap. Michigan Acad. Sci. 19: 159 (1934).

ECallerya sumatrana (Benth.) Schot, Blumea 39(1-2): 35 (1994)

Type. Indonesia, Sumatra, east coast, Boenoet [Bunut], Asahan, 4 January 1925, [Harry Stanley] Yates 1261, MICH1 104344 (MICH, lecto.! designated here); K000496724 (K, isolecto.!); A00063378 (A, isolecto.!), BO-1246847 (BO, isolecto.!); P03347972 (P, isolecto.!); US00344738 (US, isolecto.!); US00997123 (US, isolecto.!)

Distribution. Indonesia (Sumatra).

Habitat. In lowland forest from sea level to $100 \mathrm{~m}$.

\section{Kanburia J.Compton, Mattapha, Sirich. \& Schrire, gen. nov.} urn:lsid:ipni.org:names:77198977-1

Diagnosis. The two species of Kanburia share some characters with Whitfordiodendron, notably sericeous standards with narrow ridge callosities. In Kanburia bracteoles are absent (vs. present in Whitfordiodendron), keel petals are glabrous (vs. densely sericeous), pods linear, compressed, 1-1.8 cm wide (vs. inflated, ovoid, 2-2.5 cm wide). Kanburia also shares some characters with Callerya s.str. but Kanburia lacks bracteoles, the flowers are much smaller, $1-1.4 \mathrm{~cm}$ long (vs. 1.6-2.8 cm), the wings equal the keel in length (vs. much shorter than keel) and the style is shorter, 1-3 mm long (vs. 6-9 mm long). Molecular evidence for the segregation of this genus is compelling (see figs 3 and 4 in Sirichamorn et al. (2016: 45, 46, 48).

Type species. Kanburia chlorantha (Mattapha \& Sirich.) J.Compton, Mattapha, Sirich. \& Schrire $\equiv$ Callerya chlorantha Mattapha \& Sirich.

Genus description. Robust, twining woody vines. Stems pubescent when young, terete. Leaves evergreen, chartaceous and glabrescent (pubescent in $K$. tenasserimensis) when young, sparsely pubescent or glabrous when mature, imparipinnate with 5 leaflets, rachis $2.5-6 \mathrm{~cm}$ long. Stipules $1-4.5 \mathrm{~mm}$ long, acicular or ovate, caducous. Stipels 
1-2 mm long, linear, persistent. Leaflets $5-15 \times 2-11 \mathrm{~cm}$, elliptic to ovate, sparsely pubescent or glabrescent above and below especially along veins, apex acute, margins entire, base cordate or cuneate. Inflorescence a lax many-flowered, erect or pendulous terminal panicle $20-30 \mathrm{~cm}$ long, peduncle thinly pubescent. Flowers $10-15 \mathrm{~mm}$ long, emerging from June to August (August - October in K. tenasserimensis). Floral bracts 1-5 mm long, caducous, elliptic to ovate. Bracteoles absent. Pedicels 2-6 mm long, sericeous. Calyx 4-6 mm long, campanulate, brownish green (K. chlorantha) or purplish brown (K. tenasserimensis), externally densely sericeous, five lobed, lower teeth $1-1.5 \mathrm{~mm}$ long, deltoid. Standard 8-10 $\times 8-10 \mathrm{~mm}$, broadly obovate to orbicular, inner surface pale green $(K$. chlorantha) dark purple or maroon (K. tenasserimensis), nectar guide dark green $(K$. chlorantha) or pale yellow, back of standard sericeous, apex acute or emarginate. Callosities of ridge type. Wing petals $7-8 \times 3 \mathrm{~mm}$, glabrous or with a few scattered hairs, semi-pandurate with basal claws 1-2.5 mm long. Keel petals 6-7 × 3-3.5 mm, glabrous, united into a long, navicular cup, apex obtuse. Stamens diadelphous, nine fused together, the vexillary one free, all curved upwards at apex. Ovary sericeous, style 1-3 mm long, curved upwards at apex, stigma punctate. Pods 5-13 x 1-1.8 cm, flattened, linear, dehiscent, exocarp surface glabrescent, subseptate. Seeds 1-6, 10-12 $\times$ 9-11 × 3-5 mm, lenticulate, smooth, dark brown, hilum 1-2 ×0.5-1 mm elliptic.

Distribution. Thailand: Kanchanaburi, Suphan Buri, Tak and Ratchaburi [Changwats]. To be expected along the Tenasserim range between Thailand and Myanmar.

Etymology. The generic name refers to Kanburi, the old Siamese name for Kanchanaburi Province in western Thailand where the type species $K$. chlorantha was discovered.

\section{Key to species of Kanburia}

1 Floral bracts $3-5 \times 1-1.8 \mathrm{~mm} \pm$ equal to flower buds; flowers whiteish to green, nectar guide on standard green K. chlorantha

- $\quad$ Floral bracts $1-1.5 \times 0.5 \mathrm{~mm}$, shorter than flower buds; flowers purplish brown, nectar guide on standard yellow.

K. tenasserimensis

Kanburia chlorantha (Mattapha \& Sirich.) J.Compton, Mattapha, Sirich. \& Schrire, comb. nov.

urn:lsid:ipni.org:names:77198997-1

E Callerya chlorantha Mattapha \& Sirich., Phytotaxa 263(1): 44. Type: Thailand, Kanchanaburi, Sai Yok district, 20 June 2014, Phutthai \& Sirichamorn 2014-1 (BKF, holo.!); (BK, iso.!); (K, iso.!); (KKU, iso.!); (L, iso.!); (QBG, iso.!)

Illustrations. Sirichamorn et al. in Phytotaxa 263(1): 45 fig. 2 [A-C]; 46 fig. 3 (2016). Plate 1E.

Distribution. Thailand (Kanchanaburi, Tak).

Habitat. In open sites in lowland thickets and degraded bamboo forest 100 to $200 \mathrm{~m}$. 
Kanburia tenasserimensis (Mattapha \& Sirich.) J.Compton, Mattapha, Sirich. \& Schrire, comb. nov.

urn:lsid:ipni.org:names:77198998-1

三 Callerya tenasserimensis Mattapha \& Sirich., Phytotaxa 263(1): 47. Type: Thailand, Ratchaburi, Suan Phueng district, Khoa Chon (Khao Chan) waterfall, 12 September 2015, Sirichamorn 2015-13 (BKF, holo.!); (BK, iso.!); (K, iso.!); (KKU, iso.!); (L, iso.!)

Illustrations. Sirichamorn et al. in Phytotaxa 263(1): 45 fig. 2 [D-F]; 48 fig. 4 (2016). Plate 1F.

Distribution. Thailand (Ratchaburi, Suphan Buri).

Habitat. In open sites in dry deciduous and bamboo forest at 200 to $400 \mathrm{~m}$.

\section{Afgekia Craib, Bull. Misc. Inform. Kew 1927(9): 376 (1927), emend. nov. J.Compton \& Schrire}

Diagnosis. Both species of Afgekia have two pairs of callosities on the standard petal (vs. one pair in all other genera). Stipules are the longest in the tribe 10-25 mm long (vs. 3-12 mm in Sarcodum; 5-10 mm in Endosamara). Floral bracts are also the longest 30-45 mm (vs. 6-20 mm in Sarcodum). Bracteoles are absent (vs. present in Padbruggea). The oblong pods are 6-9 cm long, smooth with a velutinous indumentum (vs. 10-25 mm long, obovate or oblong, coarsely ridged and tomentose in Padbruggea). Seeds are flattened ellipsoid or flattened orbicular 8-13 mm thick (vs. ovoid or oblong 15-30 mm thick in Padbruggea).

Type species. Afgekia sericea Craib.

Genus description. Scrambling climbers to $10-20 \mathrm{~m}$. Stems green becoming brown, terete, densely sericeous. Leaves with 9-17 leaflets, evergreen, finely sericeous above and densely silvery sericeous below, imparipinnate, rachis $8-25 \mathrm{~cm}$ long. Stipules 10-25 mm long, linear-lanceolate, persistent. Stipels 3-5 mm long, acicular. Leaflets $3-8 \times 2-3 \mathrm{~cm}$, ovate to elliptic, apex softly mucronate, margins finely ciliate, base cuneate or obtuse. Inflorescence an erect leafy raceme $30-70 \mathrm{~cm}$ long, peduncle silvery sericeous. Flowers $23-25 \mathrm{~mm}$ long, emerging from June - November. Floral bracts 15-35 mm long, lanceolate, apex attenuate, densely pubescent, deep pink (purple in A. mahidoliae), caducous. Bracteoles absent. Pedicels 7-20 mm long, sericeous. Calyx 5-7 $\times 5 \mathrm{~mm}$ campanulate, green, ivory, pinkish or purple and sericeous externally, five lobed, teeth pubescent and long acuminate, upper 2 teeth 4-8 $\mathrm{mm}$ long, lower 3 teeth with central tooth longest 15-17 mm, laterals 7-9 mm long. Standard 15-28 × 20-25 mm, ovate-elliptic, cream suffused with pale or dark pink or purple, sometimes streaked at base, nectar guide pale or dark yellow or greenish, back of standard densely sericeous, apex acute. Callosities in two series, a small papillate pair near the base beneath a much larger corniculate pair either side of the midline. Wing petals 20-25 × 5-7 mm, deep pink or purple, slightly falcate, glabrous except for a ciliate fringe below the apex, more or less equal in length to the keel, obovate, apex acute conjoined over the keel, basal claws $3 \mathrm{~mm}$ long, with either one (A. mahidoliae) or two auricles (A. seri- 
cea). Keel petals 23-26 × 7-15 mm, white, sericeous externally, broadly navicular, claws 3-8 mm long, apex rounded. Stamens diadelphous, nine fused together, the vexillary one free but lying adnate to the others, all curved upwards at apex, glabrous (basally tufted in A. sericea). Ovary sericeous, style 1-3 mm long, glabrous, (tufted apically in A. mahidoliae) curved upwards at apex, stigma punctate. Pods 6-15 × 3-4 cm, inflated, elliptic or oblong, dehiscent, surface smooth to slightly wrinkled, velutinous, subseptate. Seeds 2-3, flattened-ellipsoid or orbicular, 15-25 × 10-14 × 8-13 mm, hilum strap-shaped 15-30 mm long. Plate $2 \mathrm{~A}-\mathrm{C}, \mathrm{E})$

Etymology. Afgekia commemorates Arther Francis George Kerr (1877-1942), Irish physician and pioneering botanist in Thailand.

\section{Key to species of Afgekia}

1 Leaves with 9-11 leaflets; standard petals purple; wing petals blue purple with 1 claw at base; style sericeous ....................................... mahidoliae

- $\quad$ Leaves with 13-19 leaflets; standard petals cream and pink; wing petals redpurple with 2 claws at base; style glabrous

A. sericea

\section{Afgekia sericea Craib, Bull. Misc. Inform. Kew 1927(9): 377 (1927)}

Type. Thailand, "Korat [Nakhon Ratchasima], August 1924, pink flowers, climber, Tua pep chang”, Anuwat 4 [also called Phya Anuwat Wanaraks], K000881060 (K, holo.!)

Illustrations. Lôc and Vidal in Fl. Cambodge, Laos \& Vietnam 30: 14, t. 2 [1011] (2001); Sirichamorn, MSc Thesis Pl. 4.2 [A1-A3] (2006). https://florafaunaweb. nparks.gov.sg/special-pages/plant-detail.aspx?id=1301 (Plate 2B, C).

Distribution. Laos; Thailand (Saraburi, Buri Ram, Chaiyaphum, Nakhon Ratchasima, Prachin Buri); Vietnam.

Habitat. Open sites in dry evergreen forest scrambling over shrubs at 200 to $520 \mathrm{~m}$.

\section{Afgekia mabidoliae B.L.Burtt \& Chermsir., Notes Roy. Bot. Gard. Edinburgh 31: 131 (1971)}

Type. Thailand, Bankhen, Bangkok cultivated plant from Kanchanaburi, 9 September 1968, C. Chermsirivathana 997 (BK, holo.); E00275431 (E, iso.!); E00275432 (E, iso.!)

Illustrations. Lôc and Vidal in Fl. Cambodge, Laos \& Vietnam 30: 13 (2001); Sirichamorn, MSc Thesis Pl. 4.2 [B1-B3] (2006). https://florafaunaweb.nparks.gov.sg/ Special-Pages/plant-detail.aspx?id=3478 (Plate 2A, E).

Distribution. Thailand (Kanchanaburi).

Habitat. Open sites in tropical forest scrambling among shrubs at 200 to $400 \mathrm{~m}$. 
Clade D - Padbruggea and Austrocallerya

(Fig. 1; Suppl. material 1: Figs S1-S6)

\section{Padbruggea Miq., Fl. Nederl. Ind. 1(1): 150 (1855), emend. nov. J.Compton \& Schrire}

Diagnosis. Padbruggea has robust panicles with the peduncle and lateral axes densely brown velutinous (vs. robust panicles with peduncle and lateral axes finely grey-pubescent in Austrocallerya). Padbruggea has inflated, $4.5-11 \mathrm{~cm}$ wide, oblong or obovoid coarsely ridged fruits (vs. inflated, $3-5.2 \mathrm{~cm}$ wide, fusiform, finely ridged or striate and torulose pods in Austrocallerya). Austrocallerya has arching type callosities on the standard petals vs. large papillate callosities in Padbruggea filipes or ridge type callosities in $P$. dasyphylla and P. maingayi. Padbruggea is distributed from southern China, IndoChina, Indonesia, Malaysia, Thailand to Myanmar whereas Austrocallerya occurs in Australia, New Guinea, and some of the adjacent Pacific islands as far south as New Caledonia and Norfolk Island.

Type species. Padbruggea dasyphylla Miq.

Genus description. Scrambling climbers reaching $15-25 \mathrm{~m}$. Stems dark green becoming brown, terete, densely brown pubescent when young, glabrescent. Leaves with 9-19 leaflets, evergreen, pubescent above and below when young, glabrescent or sparsely pubescent at maturity, imparipinnate, rachis 10-30 cm long. Stipules $1-8 \mathrm{~mm}$ long, ovate or lanceolate, caducous, pubescent or sericeous externally, glabrous internally. Stipels $1-3 \mathrm{~mm}$ long, filiform, glabrous or setaceous (absent in P. filipes). Leaflets $5-12 \times 2-3 \mathrm{~cm}$, oblong, ovate or elliptic, apex acute or acuminate, margins glabrous or ciliate, base rounded or obtuse. Inflorescence an erect terminal, sometimes leafy or cauliflorous panicle $7-35 \mathrm{~cm}$ long, peduncle silvery or brown tomentose. Flowers $13-25 \mathrm{~mm}$ long, emerging from April - June (July-August $P$. filipes). Floral bracts 5-25 mm long, linear-lanceolate, ovate or cupuliform, apex acute to acuminate, densely pubescent externally and internally, margin ciliate, green, (pink or purple in P. filipes), caducous. Bracteoles 3-6 mm long, narrowly lanceolate, caducous (linear $1 \mathrm{~mm}$ long $P$. filipes). Pedicels 4-7 mm long, densely pubescent $(15-25 \mathrm{~mm}$ long, sericeous in P. filipes). Calyx $4-5 \times 5 \mathrm{~mm}$ campanulate, green or purple, sericeous externally, glabrous internally, five lobed, teeth acute 1-6 mm long, margins ciliate. Standard 14-25 × 14-22 mm, orbicular, inner surface lilac or pinkish, nectar guide yellow, back of standard pubescent, apex emarginate, callosities of ridge type (papillate in P. filipes). Wing petals 13-20 × 8-11 mm, violet or pinkish, slightly falcate, glabrous, more or less equal in length to the keel, elliptic, apex rounded, basal claw 4-5 mm long. Keel petals 10-15 $\times 3-10 \mathrm{~mm}$, white, densely hairy along lower margin externally (glabrous in P. filipes); navicular, claw 3-10 mm long, apex acute or rounded. Stamens diadelphous, nine fused together, the vexillary one free, all curved upwards at apex, glabrous. Ovary densely pubescent or sericeous, style 3-4 mm long, glabrous, tufted at base, curved upwards at apex, stigma punctate. Pods 10-25 × 5-11 cm, inflated, obovoid, compressed-cuboid or oblong, dehiscent, surface coarsely ridged to rugose, velutinous, subseptate. Seeds $1-2$, elliptic-ovoid or prolate-spheroid, $50-80 \times 40-45 \times 30-45 \mathrm{~mm}$, hilum strap-shaped 16-36 mm long. 


\section{Key to species of Padbruggea}

1 Floral bracts $20 \times 10 \mathrm{~mm}$; pedicels $15-25 \mathrm{~mm}$ long; callosities papillate; pods obovoid P. filipes

- $\quad$ Floral bracts 4-8 $\times 1-4 \mathrm{~mm}$; pedicels $3-15 \mathrm{~mm}$ long; callosities ridged; pods oblong 2

2 Pedicels $5-15 \mathrm{~mm}$ long; floral bracts $8 \times 4 \mathrm{~mm}$, linear-lanceolate; bracteoles 1-3 mm long; leaves 9-11 foliolate; leaflets elliptic-oblong, apex acute, sparsely pubescent or glabrescent, margins not revolute P. dasyphylla

- $\quad$ Pedicels 3-4 mm long; floral bracts $4 \times 1 \mathrm{~mm}$, linear; bracteoles 3-6 mm long; leaves 13-17 foliolate; leaflets elliptic, apex obtuse, densely brown villose on both surfaces, margins revolute

P. maingayi

\section{Padbruggea dasyphylla Miq., Fl. Ned. Ind. 1(1): 150 (1855)}

= Millettia oocarpa Prain ex King, J. Asiat. Soc. Bengal Pt. 2, Nat. Hist. 66(2): 92 (1897). Type: Malaysia, Perak, Batu Togoh 250 ft. "climber, June 1888”, [Leonard] Wray 2141, K000881018 (K, lecto.! designated here); Paratypes: Malaysia, Perak, [Revd. Benedetto] Scortechini 429, BM001217299 (BM!); K000881017 "Derris n. sp. Sect Aganope" Herb. Mus. Perak (K!); (CAL, x 2).

Type. Indonesia, Java, "A ki kialys, leguminos, Sallak, Java. Derris tomentella Bl." [Blume scripsit] Herb. Lugd. Batav. 908.114-1723, April 1825, Blume s.n., L1978535 (L, lecto.! designated here); Herb. Lugd. Batav. 908.114-1724, L1978536 (L, isolecto.!) $\equiv$ Millettia dasyphylla (Miq.) Boerl., Handl. Fl. Ned. Ind. 1(2): 349 (1890) $\equiv$ Callerya dasyphylla (Miq.) Schot, Blumea 39(1-2): 20 (1994)

Note. Schot (1994: 20) selected a specimen at K (and a "type" at L), collected by Thomas Horsfield in Java, as the type of Padbruggea dasyphylla, however, the thirteen small leaflets per leaf, obtuse apices, revolute margins and dense, brown pubescence suggests this is Padbruggea maingayi (see below) not $P$. dasyphylla. Moreover, Miquel's protologue only specified as type "Derris? tomentella Blume in Herb. L. B." with the additional information "Banjoemas [Banyumas], op den Salak, in de bosschen bij Tapos, $1000 \mathrm{ft}$ ". A search in the Herbarium at L (G. Thijsse pers. comm.) has uncovered two Blume specimens from Java annotated "Derris tomentella Bl.”. Carl Ludwig Blume was director of the Dutch East Indies Botanic Garden at Buitenzorg [Bogor] from 1823-1826. He collected these specimens on nearby Mount Salak and around the village of Tapos in April 1825. One of these two specimens is used to lectotypify the name here. The annotation "A ki kialys" may refer to a local name for the plant.

Illustration. Lectotype sheet of Padbruggea dasyphylla at (L); L1978535.

Distribution. Indonesia (Borneo: Kalimantan, Java, Sumatra); Malaysia (Peninsula, Sarawak); Thailand. 
Habitat. Clambering over shrubs and up trees in open sites in evergreen forest at 50 to $1500 \mathrm{~m}$.

Etymology. Padbruggea commemorates Dr Robbert Padbrugge (1687-1691) Governor of Ambon for the Dutch East India Company.

\section{Padbruggea maingayi (Baker) Dunn, Bull. Misc. Inform. Kew 1911(4): 198 (1911)}

三 Millettia maingayi Baker, Fl. Brit. India 2: 110 (1879). Type: Singapore, 1867-1868, [Alexander Carroll] Maingay 2757, K000881019 (K, lecto.! designated here; see note below)

三Phaseoloides maingayi (Baker) Kuntze, Revis. Gen. Pl. 1: 201 (1891)

Nomenclatural note. There is a sheet at Kew, K000881019, with two different collections by Maingay. One has two mature pods, a leaflet and a few scraps of stem, the other has several leaflets and bits of stem. There are two labels attached at the bottom of the sheet; one states "Herbarium A.C.Maingay 2757, Singapore, 1867-1868, apparently a climber, no duplicates of this interesting sp.". The other has "Herbarium of the late A.C.Maingay 605, Malaya, distributed at the Royal Gardens, Kew, 1871". There is, however, no indication as to which collection represents Maingay 2757 and which might be Maingay 605. Baker in his protologue mentions "Singapore, Maingay" and described the 15 or more leaflets, rounded at both ends and the oblong, velvety pod traversed with deep longitudinal grooves. Since Baker described both fruit and leaves we have inferred that the left-hand fruiting specimen is Maingay 2757 and thereby have selected it to lectotypify the name.

Dunn (1911: 197) in his key to the species Padbruggea dasyphylla and P. maingayi stated that $P$. dasyphylla had leaflets with revolute margins and was densely tomentose below whereas $P$. maingayi did not have leaflets with revolute margins and was nearly glabrous below. Our examination of type material of both species has found that the reverse is the case as indicated in our key to the species.

Illustration. http://powo.science.kew.org/taxon/urn:lsid:ipni.org:names:21897-1 ( $5^{\text {th }}$ image).

Distribution. Indonesia (Java); Malaysia (Peninsula); Singapore.

\section{Padbruggea filipes (Dunn) Craib, Fl. Siam. 1: 397 (1928)}

Type. "China, Yunnan, Szemao [Simao], east mountain forests $6700 \mathrm{ft}[1520 \mathrm{~m}$ ], long climber fls pale purple" presented by Dr A. Henry in 1900, Henry 11,610, K000881062 (K, lecto.! designated here); US00003999 (US, isolecto.!); (CAL, isolecto.); MO-022362 (MO, isolecto.!) 
Key to varieties of Padbruggea filipes

1 Leaflets chartaceous, silvery pubescent only when young, glabrescent var. filipes

- $\quad$ Leaflets coriaceous, brown tomentose, persistent var. tomentosa

\section{Padbruggea filipes var. filipes}

三Adinobotrys filipes Dunn, Bull. Misc. Inform. Kew 1911(4): 195 (1911)

三Whitfordiodendron filipes (Dunn) Dunn, Bull. Misc. Inform. Kew 1912: 364 (1912b)

三 Afgekia filipes (Dunn) R.Geesink, Leiden Bot. Ser. 8: 77 (1984)

Illustrations. Lôc and Vidal in Fl. Cambodge, Laos \& Vietnam 30: 10, t. 2 [1-9] (2001); Sirichamorn, MSc Thesis Pl. 4.2 [C1-C3] (2006). https://baike.baidu.com (in Home Page enter Whitfordiodendron filipes) (Plate 2H, I).

Distribution. China (Guangxi, Yunnan); Laos; Myanmar; Thailand; Vietnam.

Habitat. In open sites climbing over scrub in thickets on dry forested hillsides at 700 to $1700 \mathrm{~m}$.

Padbruggea filipes var. tomentosa (Z.Wei) J.Compton, Sirich. \& Schrire, comb. nov. urn:Isid:ipni.org:names:77198999-1

EWhitfordiodendron flipes var. tomentosum Z.Wei, Acta Phytotax. Sin. 27(1): 75 (1989). Type: China, Yunnan, Yan-shan hsien, Bar-garh, alt. $1100 \mathrm{~m}$. in bushes 1 Nov. 1939, C.W.Wang 84801, PE00320036 (PE, holo.!); (IBSC, iso.); (KUN, iso.) 三 Afgekia filipes var. tomentosa (Z.Wei) Y.F.Deng \& H.N.Qin, Ann. Bot. Fenn. 42(2): $133(2005)$

Distribution. China (Guangxi, Yunnan).

\section{Austrocallerya J.Compton \& Schrire, gen. nov.} urn:Isid:ipni.org:names:77198978-1

$\equiv$ Millettia Sect. Austromillettia Dunn, J. Linn. Soc., Bot. 41: 135 (1912a)

Note. Dunn (1912a) recognised the distinctiveness of the Australasian species when he placed all three in his Millettia Sect. Austromillettia Dunn. He noted the single flowers as opposed to flowers in pairs (sometimes more than two branching from the same place on the inflorescence axis in other Millettia spp.), and the terete woody nature of the pods (Dunn 1912a: 135, 138, 140). 
Diagnosis. Austrocallerya comprises three Australasian species with glabrous or finely pubescent young leaves and stems (vs. these densely brown tomentose in Padbruggea, see Table 4 and Fig. 6). The robust paniculate inflorescences are more erect than those in Padbruggea and the flowers have very broad standard petals with a recessed dividing midline. Either side of the midline is an arch callosity which forms a short crescent arching over the staminal sheath (vs. papillate or ridge callosities in Padbruggea). The pods are fusiform (vs. obovoid or compressed-cuboid in Padbruggea), torulose and with either longitudinal striations and furrows (A. megasperma), or with irregular fine striations (A. australis) or smooth (A. pilipes), the surface in all cases being densely velutinous or pubescent. The pods of Austrocallerya can be distinguished from those of Padbruggea, which are also densely velutinous, by their outline. Padbruggea pods are either obovoid (in P. filipes) or oblong with a prominent dorsal midline flanked by two large flanges meeting at the apex (P. dasyphylla). The pods in Austrocallerya are 30-52 mm wide (vs. $40-110 \mathrm{~mm}$ wide in Padbruggea). The 2-10 seeds in Austrocallerya are oblong, ellipsoid or globose, frequently with one side compressed within the pod (vs. 1-2 elliptic-ovoid or prolatespheroid seeds which may also be laterally compressed in Padbruggea). In Austrocallerya the strap-shaped hila are 16-30 × 2-4 mm, (vs 16-40 × 5-10 mm in Padbruggea). Fig. 6 .

Type species. Austrocallerya australis (Endl.) J.Compton \& Schrire $\equiv$ Pterocarpus australis Endl.

Genus description. Three species of robust, twining woody vines scrambling from 2-20 m in height. Stems grey, tan or reddish brown, terete, mature stems and branches exhibiting a flaky peeling bark, young growth sericeous-pubescent becoming glabrous. Stipules 1.5-6 mm long, deltoid or filiform, sericeous, caducous (persistent in A. megasperma). Stipels 1-4 mm long, filiform, sericeous, persistent or caducous (absent in $A$. pilipes). Leaves evergreen, coriaceous and nitid when mature, imparipinnate with 5-19 leaflets, rachis $7-25 \mathrm{~cm}$ long, pubescent becoming glabrous. Leaflets $3-14 \times 1-7 \mathrm{~cm}$, elliptic, narrowly oblong or obovate, upper surface glabrous, lower surface with scattered hairs especially along veins, apex obtuse, retuse, emarginate, acute, acuminate or caudate, margins entire, base truncate, obtuse or cuneate. Inflorescence a robust manyflowered terminal panicle $6-40 \mathrm{~cm}$ long, sericeous or pubescent. Flowers $11-16 \mathrm{~mm}$ long, emerging from September to December (in A. australis from December to April but in New Guinea from June to October). Floral bracts 2-7 mm long, (8-15 mm in A. pilipes), white, green or pink, sericeous, cupuliform or linear or ovate to narrowly ovate, caducous. Bracteoles 1-7 mm, at top of pedicel, linear, sericeous, acuminate, caducous. Pedicels 3-21 mm long, pinkish, sericeous or pubescent. Calyx 3-7 4-10 mm, campanulate, yellowish or purple, glabrous internally, sparsely pubescent or sericeous externally, five lobed, upper teeth acute, 4-5 mm long, lower teeth 3-5 mm long, acuminate. Standard 12-22 × 11-18 mm, orbicular or broadly elliptic, whitish, reddish-purple, mauve, lilac, pink or purple, deflexed backwards near the base, apex with a short mucro, lamina veined, nectar guide yellow, greenish yellow or lime green, radiating up the centre of the lamina from the base, back of standard pubescent, callosities arched over the staminal sheath and divided centrally by a linear sinus, each half forming an arch. Wing petals 11-14 × 5-6 mm, purple or maroon, equalling keel in length, glabrous, each semi-pandurate with basal claws $2-3 \mathrm{~mm}$ long. Keel petals 
11-14 × 4-6 mm, dark reddish, purple or maroon (white with purple apices in $A$. megasperma), glabrous or upper margin ciliate at base (A. australis), petals united into a semi-pandurate cup, apex obtuse. Wings and keel petals spreading after anthesis. Stamens diadelphous, nine fused together, the vexillary one free, all curved upwards at apex, glabrous. Ovary sericeous, style 3-5 mm long, curved upwards at apex, stigma punctate. Pods 7-23 × 3-5.2 cm, fusiform, inflated, torulose, tardily dehiscent, exocarp finely ridged, longitudinally striate (smooth in A. pilipes), surface velutinous, endocarp chartaceous, the seeds in hollow cavities, subseptate. Seeds (1 -) 2-6, ellipsoid, broadly ovoid to squarish, smooth, brown or orange-brown $12-43 \times 12-42 \times 12-41 \mathrm{~mm}$, sometimes compressed laterally inside the pod, hilum $16-30 \mathrm{~mm} \times 2-4 \mathrm{~mm}$, strapshaped. Fig. 6.

Distribution. Australia (New South Wales, Queensland); Papua New Guinea (Bougainville Island, New Britain Island); New Caledonia; Cook Islands.

Habitat. In rainforest or in dry forest from sea level to $1600 \mathrm{~m}$, climbing up trees and over shrubs.

Etymology. The generic name reflects the southern hemisphere distribution of the genus, austro - "australis" = south (Latin) and "callerya" a reference to their former generic placement and affinity.

\section{Key to species of Austrocallerya}

1 Floral bracts $2-7 \times 0.5-2 \mathrm{~mm}$, linear or narrowly ovate not enclosing flower buds prior to anthesis; bracteoles $1-3 \times 0.5-1 \mathrm{~mm}$; pod surface with longitudinal ridges .2

- $\quad$ Floral bracts 8-15 $\times 8-12 \mathrm{~mm}$, cupuliform, enclosing flower buds prior to anthesis; bracteoles 5-7 × 1-2 mm; pod surface glabrous or finely tesselated

A. pilipes

2 Floral bracts 6-7 $\times 2 \mathrm{~mm}$; pod surface deeply ridged or grooved; seeds dark brown

A. megasperma

- $\quad$ Floral bracts $2-4 \times 0.5-1 \mathrm{~mm}$; pod surface finely and shallowly ribbed; seeds orange-brown

A. australis

\section{Austrocallerya australis (Endl.) J.Compton \& Schrire, comb. nov.} urn:Isid:ipni.org:names:77199026-1

E Pterocarpus australis Endl., Prodr. Fl. Norf.: 94 (1833). Type: Australia, Norfolk Island, "In Insula Norfolk, Ferd. Bauer", 1804-1805, F.Bauer s.n., W0046224 (W, lecto.! designated here); W0046223 (W, isolecto.!); W0046225 (W, isolecto.!);

"Norfolk Island, Bauer Hb. Brown" K000880984 (K, isolecto.!)

EMillettia australis (Endl.) Benth. Pl. Jungh. [Miquel] 2: 250 (1852) 


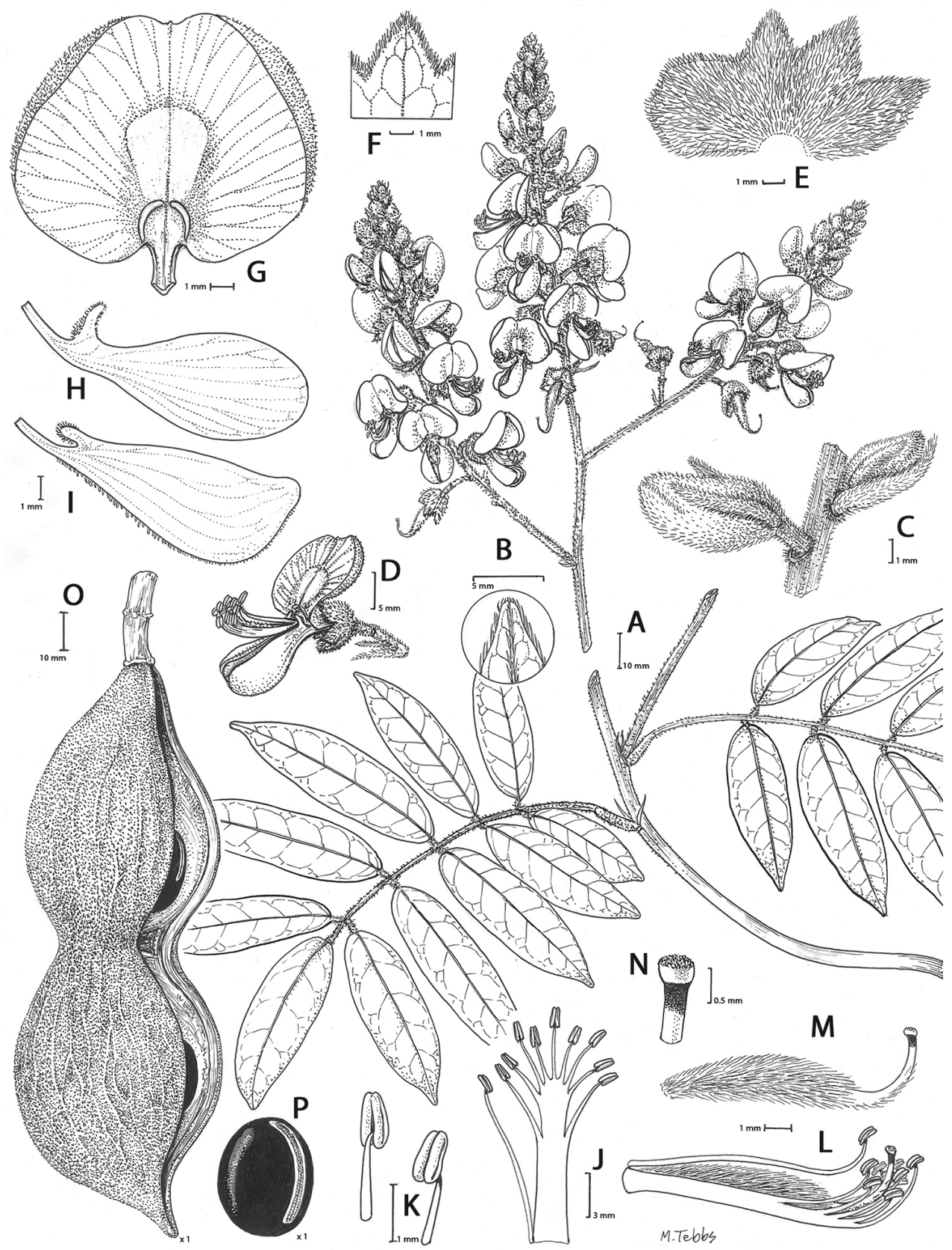

Figure 6. Austrocallerya australis (Endl.) J.Compton \& Schrire. A Habit B leaf and detail of leaflet apex $\mathbf{C}$ flower buds with bract and 2 bracteoles $\mathbf{D}$ flower $\mathbf{E}$ calyx external surface $\mathbf{F}$ calyx detail of inner surface $\mathbf{G}$ standard petal inner surface $\mathbf{H}$ wing petal I keel petal $\mathbf{J}$ staminal column ventral view $\mathbf{K}$ stamens dorsal and ventral views $\mathbf{L}$ staminal column lateral view $\mathbf{M}$ ovary lateral view $\mathbf{N}$ stigma $\mathbf{O}$ pod $\mathbf{P}$ seed angled lateral view (all from Martin 1392). Drawn by Margaret Tebbs. 
三Wisteria australis (Endl.) F.Muell. Second Systematic Census of Australian Pl.: 68 (1885)

E Kraunhia australis (Endl.) Greene, Pittonia 2(10): 175 (1891)

三 Callerya australis (Endl.) Schot, Blumea 39(1): 16 (1994)

= Millettia maideniana F.M.Bailey, Queensland Bot. Bull. Agric. 5: 12 (1892). Type:

Australia, New South Wales, "Port Macquarie, Bean tree, 16 ins diameter, 25-30 ft high, Nov. 1891,", J.H.Maiden s.n, BRI-AQ22886 (BRI, holo.!) 三 Wisteria maideniana (F.M.Bailey) C.Moore, Handbook Fl. New South Wales: 517 (1893).

= Callerya neocaledonica I.C.Nielsen \& Veillon, Adansonia ser. 3, 27(1): 82 (2005).

Type: Nouvelle-Calédonie, Dumbéa, Nakutakoin, western slope of Pic Jacob, c. 150 m, 15 January 1992, J.M. Veillon 7466, P00625937 (P, holo.!); P00629538 (P, iso.!); P00625939 (P, iso.!); (AAU, iso.), NOU006199 (NOU, iso.).

Illustration. Harden in Flora of New South Wales 2: 615 (2000). http://www.floragreatlakes.info/html/rfspecies/callerya.html (Callerya australis).

Distribution. Australia (Norfolk Island, New South Wales, Queensland); Bougainville Island; Cook Islands; New Britain Island; New Guinea; New Caledonia.

Habitat. In rainforest climbing in thickets on forested hillsides at 300 to $1600 \mathrm{~m}$.

\section{Austrocallerya megasperma (F.Muell.) J.Compton \& Schrire, comb. nov.} urn:Isid:ipni.org:names:77199027-1

三Wisteria megasperma F.Muell., Fragmenta Phytographiae Australiae 1: 10 (1858).

Type Australia, Queensland, "Ad rivulos sylvaticos fluvii Pine River prope sinum

Moreton Bay", Fvon Mueller \& W.Hill s.n., MEL2144485 (MEL, lecto.!, designated by Compton (2016)); MEL2144484 (MEL, isolecto.!)

三 Millettia megasperma (F.Muell.) Benth., Fl. Austral. 2: 211 (1864)

三 Kraunhia megasperma (F.Muell.) Greene, Pittonia 2(10): 175. (1891)

三 Phaseoloides [Phaseolodes] megasperma (F.Muell.) Kuntze, Revis. Gen Pl. 1: 201 (1891)

三 Callerya megasperma (F.Muell.) Schot, Blumea 39(1): 25 (1994)

Illustrations. Harden in Flora of New South Wales 2: 615 (2000) http://www.floragreatlakes.info/html/rfspecies/callerya.html (Callerya megasperma).

Distribution. Australia (New South Wales, Queensland).

Habitat. In dry forest climbing up trees from sea level to $300 \mathrm{~m}$.

Note.

1) Schot (1994: 25) chose a specimen at K collected at Richmond River, NSW by Charles Moore, K000880988 as the type, but this was not cited in the protologue nor was it from the type locality and is therefore not original material. Another specimen K000880989 collected by Mueller and Hill from "Upper Brisbane River" was also collected from a location not cited in the protologue. 
We have chosen MEL2144485 collected by Mueller and Hill from the Pine River near Moreton Bay as cited in the protologue as lectotype.

2) Wisteria involuta Sprague (Sprague 1904: 141) was described from cultivated material at K. The material was collected from the Richmond River area of NSW, Australia, collector unknown. Sprague (1905: 3) recombined the species as Derris involuta (Sprague) Sprague the following year having seen the flat, one-seeded, winged fruits of the millettioid genus Derris Lour.

\section{Austrocallerya pilipes (F.M.Bailey) J.Compton \& Schrire, comb. nov.} urn:lsid:ipni.org:names:77199028-1

三 Millettia pilipes F.M.Bailey, Second Addenda to Third Supplement of the Synopsis of the Queensland Flora 108 (1890) [published with Catalogue of the Indigenous and Naturalised Plants of Queensland]. Type: Australia, Queensland, Cook pastoral district, "Johnstone River, Dr. Thos. L. Bancroft, large climber", 1885-1886, T.L.Bancroft s.n., BRI-AQ0022887 (BRI, holo.!); BM000810924 (BM, iso.!)

三Wisteria pilipes (F.M.Bailey) Sprague, Gard. Chron. Ser. 3., 36: 141 (1904)

Note. There is also a specimen at K collected by Frederick M. Bailey from the Johnstone River in August 1892 - K000880982. Bailey, the author of the name, had been Colonial Botanist for Queensland since 1881 but Schot's choice of this specimen as an isotype (Schot 1994: 29) is incorrect as the holotype cited in the protologue was collected by Thomas Lane Bancroft, moreover, Bailey's specimen was collected two years after the protologue was published.

Illustrations. Cooper, Australian Rainforest Fruits, a Field Guide: 175 (2013). http://keys.trin.org.au/key-server/data/0e0f0504-0103-430d-8004-060d07080d04/ media/Html/taxon/Callerya_pilipes.htm

Distribution. Australia (Queensland).

Habitat. In rainforest climbing trees and over scrub from 300 to $1200 \mathrm{~m}$.

Clade E - Wisteria

(Fig. 1; Suppl. material 1: Figs S1-S6)

14. Wisteria Nutt., Gen. Amer. Pl. 2: 115 (1818), emend. nov. J.Compton \& Schrire

Diagnosis. In Wisteria the wings remain adnate to the keel after anthesis (vs. separated from the keel in Wisteriopsis). The Asian species all have papillate callosities similar to those in Padbruggea filipes but the North American W. frutescens has small ridge type 
callosities. The pods of all Asian species are gently torulose with a velutinous surface (striated, ridged, furrowed or tessellated in Padruggea and Austrocallerya). The North American W. frutescens has straight, smooth, glabrous pods.

Type species. Wisteria frutescens (L.) Poir. $\equiv$ Glycine frutescens L.,

Genus description. Robust, twining woody vines to more than $30 \mathrm{~m}$ in height. Stems green and pubescent when young, becoming grey or reddish brown at maturity, terete. Stipules 4-8 mm long, linear, sericeous, caducous. Stipels 3-4 mm long, filiform, sericeous, caducous. Leaves deciduous, chartaceous and villose when young, glabrous when mature, imparipinnate with (7 -)9-13(-15) leaflets, rachis $4-7 \mathrm{~cm}$ long, pubescent becoming glabrous. Leaflets 2-10 × $1-5 \mathrm{~cm}$, ovate-elliptic or ellipticlanceolate, upper surface glabrous (very finely pubescent in W. frutescens), lower surface with scattered hairs along veins, apex acute, acuminate or shortly caudate, margins entire or gently sinuate, base obtuse or cuneate. Inflorescence a terminal spreading to pendulous raceme $12-30(-140) \mathrm{cm}$ long, peduncle villose or pubescent. Flowers 15-30 mm long, emerging from April to May (June to August in W. frutescens). Floral bracts 5-15(-23) $\mathrm{mm}$ long, brown or silvery chartaceous, linear or cupuliform, attenuate or caudate, caducous. Bracteoles 2-4 mm, (absent in W. frutescens) at top of pedicel, linear, acuminate, caducous. Pedicels 5-50 mm long, pubescent. Calyx 4-10 $\times$ 4-6 mm, campanulate or tubular, sparsely to densely pubescent or sericeous externally (sometimes with glandular hairs $W$. frutescens), five toothed, upper teeth acute, 3-5 mm long, lower teeth 3-6 mm long, (central tooth $-8 \mathrm{~mm}$ ) acuminate, green or white. Standard 17-28 × 19-28 mm, suborbicular, lilac or pale purple, deflexed near the base (deflexed near the middle in $W$. frutescens), apex with a short mucro or retuse, nectar guide yellow, back of standard glabrous or sparsely pubescent along margin, callosities papillate either side of the midline at base (of ridge type in $W$. frutescens). Wing petals $12-20 \times 5-8 \mathrm{~mm}$, lilac or purple, equalling keel or slightly longer, glabrous, each semi-pandurate with basal claws $2-4 \mathrm{~mm}$ long $(6-8 \mathrm{~mm}$ in W. brachybotrys). Keel petals 11-18 $\times 4-8 \mathrm{~mm}$, lilac or purple, glabrous, united into a semi-pandurate cup, apex obtuse. Stamens diadelphous, nine fused together, the vexillary one free, all curved upwards at apex, glabrous. Ovary sericeous, style $3-5 \mathrm{~mm}$ long, curved upwards at apex, stigma punctate. Pods 10-24 × 1.2-3 cm, compressed, slightly torulose, (straight in $W$. frutescens) tardily dehiscent, exocarp smooth, surface densely velutinous, endocarp pithy, the seeds in shallow cavities, subseptate. Seeds (1 -)3-6(-8), lenticular, (reniform-cuboid in W. frutescens) smooth, pale or dark brown, 8-10 × 8-12 × 2-4 mm, $(8-10 \times 4-6 \times 4-6 \mathrm{~mm}$ in $W$. frutescens $)$, hilum 1-2 $\times 1-3 \mathrm{~mm}$, elliptic. Plate $3 \mathrm{~F}-\mathrm{G}$.

Distribution. China (Anhui, Fujian, Guangxi, Hebei, Henan, Hunan, Jiangsu, Jiangxi, Shaanxi, Shandong, Shangxi, Zhejiang); Japan; Korea; east North America.

Habitat. In temperate forests from sea level to $1800 \mathrm{~m}$, climbing among trees and shrubs.

Etymology. The generic name commemorates the anatomist Professor Caspar Wistar (1761-1818), President of the American Philosphical Society. It also commemorates Caspar Wistar's cousin Charles Jones Wister (1782-1865), friend of Thomas Nuttal who was the author of the name. 


\section{Key to species of Wisteria}

1 Inflorescence cylindrical in bud; bracteoles present below calyx; papillate callosities present on standard petals; pods velutinous, slightly torulose; seeds

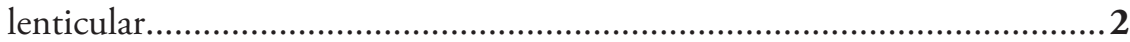

- Inflorescence conical in bud; bracteoles absent; standard petal with ridge callosities; wing petals with subulate spur-like auricles 4-6 mm long; pods glabrous with a few longitudinal undulations; seeds reniform or oblong.

W. frutescens

2 Inflorescence $12-120 \mathrm{~cm}$ long; floral bracts 5-20 × 2-12 mm, linear or ovatelanceolate, standard petal with papillate callosities; wing petals with short deltoid auricles 1-3 mm long; pods velutinous; seeds lenticular................... 3

- $\quad$ Inflorescence 8-20 cm long; floral bracts 15-23 × 5-12 mm, broadly ovate, attenuate, densely sericeous.................................................. W. brachybotrys

3 Inflorescence 18-120 cm long; floral bracts 5-12 $\times 2-6 \mathrm{~mm}$, linear, puberulent; standard petal broadly ovate, $8-16 \times 8-12 \mathrm{~mm}$............. W. floribunda

- Inflorescence 12-20 cm long; floral bracts 8-20 × 10-12 mm, ovate-lanceolate, puberulent; standard petal suborbicular, $20-30 \times 20-30 \mathrm{~mm}$ W. sinensis

Wisteria frutescens (L.) Poir., Tab. Encycl. 3: 674 (1823)

Type. [icon] "Phaseoloides, frutescens Caroliniana foliis pinnatis floribus caeruleis conglomeratis Carolina Kidney-Bean” in Miller Cat. Pl.: t. 15 (1730), (lecto.!, designated by Reveal (1997)).

Key to subspecies of Wisteria frutescens

1 Inflorescence 8-15 cm long; pedicel and calyx with simple hairs; calyx teeth subequal, all \pm acute ........................................................... subsp. frutescens

- $\quad$ Inflorescence (8-)10-30 cm long; pedicel and calyx covered externally with simple and clavate glandular hairs; upper lip of calyx with teeth acute, lower lip longer, teeth acuminate. subsp. macrostachya

\section{Wisteria frutescens subsp. frutescens}

E Glycine frutescens L., Sp. Pl. 1(2): 753 (1753)

三 Kraunhia frutescens (L.) Raf., Med. Repos. Original Essays Intelligence Phys. [hex.]

2 [vol.] 5: 352 (1808) nom. rej. (Art. 56, McNeil \& al., 2012)

$\equiv$ Apios frutescens (L.) Pursh, Fl. Amer. Sept. 2: 474 (1814)

三 Thyrsanthus frutescens (L.) Elliott, J. Acad. Nat. Sci. Philadelphia 1: 371 (1818)

$\equiv$ Phaseolus frutescens (L.) Eaton \& Wright, N. Am. Bot. ed 8: 354 (1840) 
E Kraunhia frutescens (L.) Greene, Pittonia 2: 175 (1891)

三 Phaseoloides [Phaseolodes] frutescens (L.) Kuntze, Revis Gen. Pl. 1: 201 (1891)

三 Bradlea [as Bradleya] frutescens (L.) Britton, Man. Fl. N. States \& Canada 549

(1901). Note: Bradleya is treated as a homonym of Bradlea Adans. under Art. 53.2

(Turland et al. 2018)

= Glycine caerulea Salisb., Prodromus stirpium in horto Chapel Allerton vigentium 335

(1796) [G. frutescens L. was cited].

= Wisteria speciosa Nutt., Gen. N. Amer. Pl. 2: 116 (1818) [G. frutescens L. was cited].

Illustrations. Compton in Curtis's Bot. Mag. 32 (3-4): 234, t. 2 \& 3 (2015a) https:// gobotany.newenglandwild.org/species/wisteria/frutescens/; (Plate 3F); Compton and Lane (2019: 174).

Distribution. USA (Connecticut, Delaware, Illinois, Indiana, Iowa, Maryland, Massachusetts, Michigan, Missouri, New Jersey, New York, North Carolina, Ohio, Pennsylvania, Rhode Island, Virginia, West Virginia).

Habitat. In clearings of evergreen lowland forest and along riverbanks at sea level to $650 \mathrm{~m}$.

Note. Plants from more southerly regions either side of the Appalachian mountains have previously been recognised as a separate species Wisteria macrostachya (Nutt. ex Torr. \& A.Gray) B.L.Rob. \& Fernald, Gray Man. Bot. N. U.S. ed. 7: 515. 1908. Observation of living plants and careful examination of many herbarium specimens coupled with the DNA generated results from this study have led us to conclude that there is only the single species W. frutescens representing the genus Wisteria in North America. We also conclude that there is sufficient difference between the southern plants and those from further north to recognise the southern one at the rank of subspecies. At the rank of species the name Diplonyx elegans Raf. (1817: 101) has priority over the widely used name Wisteria macrostachya (Robinson and Fernald 1908: 515), however, the combination Wisteria elegans has never been made (for a more comprehensive discussion see Compton (2015a)). Plants from the northerly range of the species (subsp. frutescens) have smaller and shorter inflorescences without (or with very few) glandular hairs on pedicels and calyces (vs pedicels and calyces covered in clavate glandular hairs in subsp. macrostachya). The teeth on the calyces of subsp. frutescens are \pm subequal (vs lower teeth much longer in subsp. macrostachya). Racemes from the colder north (subsp. frutescens) are usually considerably shorter than the elongating racemes of southerly plants (subsp. macrostachya). For a more comprehensive description of these taxa see Compton and Lane (2019).

\section{Wisteria frutescens subsp. macrostachya (Nutt. ex Torr. \& A.Gray) J.Compton \& Schrire, comb. nov.}

urn:lsid:ipni.org:names:77199029-1

$\equiv$ Wisteria frutescens var. macrostachya Nutt. ex Torr. \& A.Gray, Fl. N. Am. 1(2): 283 (1838). Type: U.S.A. Louisiana, [Louis François] Tainturier [s.n.] (fide Nuttall), PH00029452 (PH, holo.!) 
三 Wisteria macrostachya (Nutt. ex Torr. \& A.Gray) B.L.Rob. \& Fernald, Gray Man. Bot. N. U.S. ed. 7: 515 (1908) [as W. macrostachys]

三 Kraunhia macrostachya (Nutt. ex Torr. \& A. Gray) Small, Bull. Torrey Bot. Club 25: 134-135 (1898) [as K. macrostachys];

三 Bradlea [as Bradleya macrostachys] macrostachya (Nutt. ex Torr. \& A.Gray) Britton,

Man. Fl. N. States \& Canada 549 (1901). Note: Bradleya is treated as a homonym of Bradlea under Art. 53.2 ex 9 (Turland et al. 2018)

= Diplonyx elegans Raf., Fl. Ludov. 101-102 (1817). Type: Louisiana, Alexandria, J.Hale s.n., P00680371 (P, neo.!, designated by Compton (2015a)).

= Thyrsanthus floridana Croom, Amer. Journ. Sci. and Arts 25: 75 (1834). Type: "Wisteria speciosa, Thyrsanthus frutescens Ell., river banks Florida”, Chapman Herbarium [collector unknown], GH00429033 (GH, neo.!, designated by Compton (2015a)). = Glycine frutescens var. magnifica Hérincq, L'Horticulteur Français de mil huit cent cinquante et un 5: 220 (1855). Type: [Icon] L'Horticulteur Français de mil huit cent

cinquante et un 5: 220 (1855), (lecto.!, designated by Compton (2015a)

三Wisteria frutescens var. magnifica (Hérincq) André, Revue Horticole 1862: 50 (1862).

Illustrations. Compton in Curtis's Bot. Mag. 32 (3-4): 237, t. 4 \& 5 (2015a).

Distribution. USA (Alabama, Arkansas, Florida, Georgia, Kentucky, Louisiana, Mississippi, Oklahoma, South Carolina, Tennessee, Texas).

\section{Wisteria brachybotrys Siebold \& Zucc., Fl. Jap. 1: 92. t. 45. (1839)}

= Wisteria venusta Rehder \& E. H. Wilson, Publ. Arnold Arbor. 4 vol. 2: 514 (1916). Type: Japan, Hondo [Honshu], Musashi, [cultivated] Iris Garden, Kamata, 27

April 1914, E.H.Wilson 6580, US00003998 (US, lecto.!, designated by Compton and Lack (2012)); (GH, isolecto.!); K (as E.H.Wilson 6580, but dated 6 May 1914) $\equiv$ Rehsonia venusta (Rehder \& E.H.Wilson) Stritch, Phytologia 56(3): 183 (1984).

Type. Japan, "1829, Herb. de Siebold” [Fl. Jap.: t. 45.], L0176059 (L, lecto.!, designated by Compton and Lack (2012)); "Japonia coll. Dr. von Siebold donné par $\mathrm{Mr}$ Blume” P02942362 (P, isolecto.!) E Phaseoloides [Phaseolodes] brachybotrys (Siebold \& Zucc.) Kuntze, Revis. Gen. Pl. 1: 202 (1891) 三 Millettia floribunda var. brachybotrys (Siebold \& Zucc.) Matsum., Bot. Mag. (Tokyo) 16: 64 (1902) 三 Kraunhia sinensis var. brachybotrys (Siebold \& Zucc.) Makino, Bot. Mag. (Tokyo) 24: 76 (1910) $\equiv$ Kraunhia floribunda var. brachybotrys (Siebold \& Zucc.) Makino in Bot. Mag. (Tokyo) 25: 18 (1911) E Rehsonia brachybotrys (Siebold \& Zucc.) Stritch, Phytologia 56(3): 184 (1984)

Illustrations. Compton in Curtis's Bot. Mag. 32 (3-4): 305, Pl. 816 \& 817, t. 1 \& 2 (2015d); Wei and Pedley, Fl. China 10: 189, t. 223 [8] (2010). Plate 3G.

Distribution. Japan (Honshu, Kyushu, Shikoku).

Habitat. Climbing over trees and shrubs in mixed evergreen and deciduous forest and along riverbanks at sea level to $1000 \mathrm{~m}$. 
Wisteria floribunda (Willd) DC., Prodr. 2: 390 (1825), nom. cons. (Taxon 61(4): 882)

三 Glycine floribunda Willd. Sp. Pl. 3(2): 1066 (1802). Type: Japan, Aoyama, Suma Kobe city, 10 May 1967, M.Hotta 16502 (K, neo.!, designated by Compton and Lack (2012)); (P, isoneo.!); (L, isoneo.!); (E, isoneo.!)

$\equiv$ Phaseoloides [Phaseolodes] floribunda (Willd.) Kuntze, Revis. Gen. Pl. 1: 202 (1891)

三 Kraunhia floribunda (Willd.) Taub., Engler \& Prantl, Nat. Pflanzenfam. 3(3): 271. (1894)

三 Millettia floribunda (Willd.) Matsum., Bot. Mag. (Tokyo) 16: 64 (1902)

三 Rehsonia floribunda (Willd.) Stritch in Phytologia 56: 183 (1984)

= Wisteria macrobotrys Siebold ex Lemoine, Catalogue et Prix Courant no. 56: 5 (1869).

Type: [Icon] Neubert Deutsches Gartenmagazin für Garten und Blumenkunde 23: 17 (1870), (lecto.!, designated by Compton and Thijsse (2015))

$\equiv$ Wisteria sinensis var. macrobotrys (Siebold ex Lemoine) Lavallée, Énum. Arbres: 65 (1877) $\equiv$ Wisteria floribunda f. macrobotrys (Siebold ex Lemoine) Rehder \& E.H.Wilson, Publ. Arnold Arb. 4: 513 (1916)

E Kraunhia floribunda var. macrobotrys (Siebold ex Lemoine) Nash, Journal of the New York Botanical Garden 20: 14 (1919)

三 Wisteria floribunda var. macrobotrys (Siebold ex Lemoine) L.H.Bailey, Manual of Cultivated Plants ed. 1: 417 (1923).

= Wisteria multijuga Van Houtte, Fl. Serres Jard. Eur. 19: 125 (1874). Type: [Icon] Fl. Serres Jard. Eur. 19: 125 (1874) E Glycine multijuga (Van Houtte) Clémenc., Revue Horticole 46: 300 (1874), (lecto.!, designated by Compton and Lack (2012)) 三Wisteria sinensis var. multijuga (Van Houtte) H.Jaeger \& Beissn., Die Zierghölze der Gärten und Parkanlagen 425 (1889) $\equiv$ Wisteria polystachios f. multijuga (Van Houtte) Beissn. Schelle \& Zabel, Handbuch der Laubholz-Benennung 269 (1903).

Illustrations. Siebold and Zuccarini in Flora Japonica 1: t. 44 (1839) as Wisteria chinensis; Compton and Thijsse in Curtis's Bot. Mag. 32 (3-4): 350, Pl. 818, t. 2 \& 3 (2015).

Distribution. Korea; Japan (Honshu, Kyushu, Shikoku).

Habitat. Climbing over trees and shrubs in mixed evergreen and deciduous forest and in thickets at sea level to $1500 \mathrm{~m}$.

\section{Wisteria sinensis (Sims) DC. Prodr. 2: 390 (1825) 三 Glycine sinensis Sims}

Type. [Icon] Bot. Mag. 46 [n.s.4] t. 2083 (1819), (lecto.!, designated by Compton (2015b)). 


\section{Key to varieties of Wisteria sinensis}

1 Lower lip of calyx with teeth 1-2mm long, subequal, upper surface of leaves finely reticulate

var. brevidentata

- $\quad$ Lower lip of calyx with teeth 2-4 mm long, $2 \times$ longer than upper teeth, upper surface of leaves coarsely reticulate ....................................................... 2

2 Inflorescence axes, calyces and upper surface of leaves villose when young, pubescent when mature ................................................................var. villosa

- $\quad$ Inflorescence axes, calyces and upper surface of leaves sparsely pubescent when young, becoming glabrescent when mature var. sinensis

\section{Wisteria sinensis var. sinensis}

EMillettia sinensis (Sims) Benth., Pl. Junghuhn. [Miquel] 2:249 (1852) (as M. "chinensis") 三 Rehsonia sinensis (Sims) Stritch, Phytologia 56(3): 183 (1984)

= Wisteria consequana Sabine ex Loudon, The Gardener's Magazine 2: 422 (1827) [Glycine sinensis was cited].

= Wisteria praecox Hand.-Mazz., Anz. Akad. Wiss. Wien, Math-Naturwiss. Kl. 58: 177 (1921). Type: China, Hunan, "frutex volubilis fl. intense rubroviolaceis (diary no. 2357.88) prope urbem Tschangscha [Changsha] in silvulis apertis reg. subtropicae inter viam militarum Hsingaipu [Xinkaipu] et fluvium [Xiangjian river], 100m.”, legi 10 et 23 March 1918, Handel-Mazzetti 11678, WU0059306 (WU, lecto.!, designated by Compton (2015b)); WU0059305 (WU, isolecto.!); WU0059304 (WU, isolecto.!); WU19240004403 (WU, isolecto.!); Paratype: China, Hunan, "in monte Gu-schan, fl. rosei, prope urbem Tschangscha, 14 April 1918, elev. 150 m. (diary no. 2364), Handel-Mazzetti 11,623, WU0059304 (WU!).

Illustrations. Compton in Curtis's Bot. Mag. 32 (3-4): 283, Pl. 815, t. 1 \& 2 (2015); Wei and Pedley, Fl. China 10: 188, t. 223 [1 - 5] (2010).

Distribution. China (Anhui, Fujian, Guangxi, Hebei, Henan, Hubei, Hunan, Jiangsu, Jiangxi, Shaanxi, Shandong, Shanxi, Yunnan, Zhejiang).

Habitat. Climbing over trees and shrubs and along banks and over thickets 50 to $1800 \mathrm{~m}$.

\section{Wisteria sinensis var. villosa (Rehder) J.Compton \& C.Lane, Wisteria: The com- plete Guide 282 (2019)}

E Wisteria villosa Rehder, Journ. Arnold Arbor. 7: 162 (1926). Type: China, Chili, Temple of Sleeping Buddha, Wo-fu-ssu [Wofosi], Western Hills [Xishan] near Peking, received on 25 July 1923, Ralph G.Mills s.n., A00786449 (A, holo.!) 
三 Rehsonia villosa (Rehder) Stritch, Phytologia 56(3): 184 (1984)

Illustration. Wei and Pedley, Fl. China 10: 189, t. 223 [7] (2010).

Distribution. China (Hebei, Henan, Shaanxi, Shandong, Shanxi).

Habitat. Climbing over trees and shrubs 100 to $1500 \mathrm{~m}$.

\section{Wisteria sinensis var. brevidentata (Rehder) J.Compton \& C.Lane, Wisteria: The complete Guide 283 (2019)}

EWisteria brevidentata Rehder, Journ. Arnold Arbor. 7: 163 (1926). Type: China, Yunnan, Dongchuan, "Glycine, arbuste grimpant cultivé et subspontané très longs rameaux fl. violettes engrappes, jardins de Tong-tschouan alt, $2500 \mathrm{~m}$, Avril", E.E.Maire 458, A00195989 (A, lecto.! designated by Compton (2015b)); P02942331 (P, isolecto.!); K000881058 (K, isolecto.!)

三 Rehsonia brevidentata (Rehder) Stritch, Phytologia 56(3): 184 (1984)

Distribution. China (Fujian, Jiangxi, Hunan, Guizhou, Yunnan).

Habitat. Climbing over trees and shrubs 100 to $1500 \mathrm{~m}$.

\section{Acknowledgements}

Our grateful thanks are due to Gwilym Lewis (K) and Matt Lavin (MONT) for useful comments on the manuscript; to Gwil also for logistic support at Kew and permission to examine specimens and to Ian Willey for assistance with molecular data. We are extremely grateful to Alastair Culham, University of Reading, for helping us to implement this paper by raising the initial funding for the research from the Stanley Smith Horticultural Trust (UK). Thanks to Henry Noltie (E) for information on the type of Endosamara, Elspeth Haston (E) for assistance with literature and John McNeill (E) and Nick Turland (B) for answering nomenclatural queries. Thanks also to the following Curators and Directors of Botanical Gardens and Herbaria for assisting with our search for material: A, ABD, B, BM, BO, BRI, CAL, CNS, DAO, E, FI, G, GH, H, IBSC, K, KAG, L, LE, M, MICH, MO, MPU, NY, O, P, PE, US. Thanks too to Gerard Thijsse (L) for locating original material of Padbruggea dasyphylla and Charlotte Sletten Bjorå $(\mathrm{O})$ for sending images of the type material of Millettia dielsiana, Chiara Neppi (FI) for type material of Millettia bonatiana and Deby Arifiani (BO) for type material of Whitfordiodendron sumatranum. Our thanks also go to Marc Libert (GENT) and Chris Lane for providing DNA material, Koji Yonekura for searching through the collections at KAG and Young-Dong Kim for information on the distribution of Wisteriopsis in South Korea. Further thanks are due to Ailsa Holland (BRI) and Wendy Cooper (CNS) for information on the morphology of Australian Austrocallerya species. Special thanks are due to Professor Lei Duan (IBSC) for sending us fresh material of Sarcodum 
scandens from Vietnam and to Bleddyn and Sue Wynn-Jones of Crûg Farm Plants and Mark Weathington, Director, JC Raulston Arboretum, North Carolina State University for sending us material of Wisteriopsis japonica. This project would not have been possible without the aid of a grant from the Stanley Smith Trust and the generosity of the Royal Horticultural Society and the Royal Botanic Gardens, Kew.

\section{References}

Azani N, Babineau M, Bailey CD, Banks H, Barbosa AR, Pinto RB, Boatwright JS, Borges LM, Brown GK, Bruneau A, Candido E, Cardoso D, Chung K-F, Clark RP, Conceição AS, Crisp M, Cubas P, Delgado-Salinas A, Dexter KG, Doyle JJ, Duminil J, Egan AN, De La Estrella M, Falcão MJ, Filatov DA, Fortuna-Perez AP, Fortunato RH, Gagnon E, Gasson P, Rando JG, Azevedo Tozzi AMG, Gunn B, Harris D, Haston E, Hawkins JA, Herendeen PS, Hughes CE, Iganci JRV, Javadi F, Kanu SA, Kazempour-Osaloo S, Kite GC, Klitgaard BB, Kochanovski FJ, Koenen EJM, Kovar L, Lavin M, Roux M, Lewis GP, de Lima HC, López-Roberts MC, Mackinder B, Maia VH, Malécot V, Mansano VF, Marazzi B, Mattapha S, Miller JT, Mitsuyuki C, Moura T, Murphy DJ, Nageswara-Rao M, Nevado B, Neves D, Ojeda DI, Pennington RT, Prado DE, Prenner G, de Queiroz LP, Ramos G, Ranzato Filardi FL, Ribeiro PG, Rico-Arce ML, Sanderson MJ, Santos-Silva J, São-Mateus WMB, Silva MJS, Simon MF, Sinou C, Snak C, de Souza ÉR, Sprent J, Steele KP, Steier JE, Steeves R, Stirton CH, Tagane S, Torke BM, Toyama H, Cruz DT, Vatanparast M, Wieringa JJ, Wink M, Wojciechowski MF, Yahara T, Yi T, Zimmerman E, LPWG (2017) A new subfamily classification of the Leguminosae based on a taxonomically comprehensive phylogeny. Taxon 66(1): 44-77. https://doi.org/10.12705/661.3 Bailey LH, Bailey EZ (1949) Wisteria japonica in Hortus Second. Macmillan Co. New York, 770. Baldwin BG, Sanderson MJ, Porter JM, Wojciechowski MF, Campbell CS, Donoghue MJ (1995) The ITS region of nuclear ribosomal DNA - A valuable source of evidence on angiosperm phylogeny. Annals of the Missouri Botanical Garden 82(2): 247-277. https:// doi.org/10.2307/2399880

Bentham G (1842) Millettia nitida in Enumeration of the plants collected in Hong Kong by Mr. Hinds, determined and described by Mr. George Bentham esq. London Journal of Botany 1: 484 .

Bentham G (1853) Millettia in Plantae Junghuhnianae Enumaratio Plantarum quas in Insulis Java et Sumatra detexit Fr. Junghuhn [Ed Miquel FAW] vol. 2. A.W Sythoff, Leiden, J. B. Baillière, Paris.

Bentham G, Hooker JD (1865) Subtribus Tephrosieae in Genera plantarum Vol. 1(2). Lovell Reeve \& Co.; Williams \& Norgate, London, 444-445.

Burtt BL, Chermsirivathana C (1971) A second species of Afgekia (Leguminosae). Notes from the Royal Botanic Garden Edinburgh 31(1): 131-132.

Cardoso D, De Queiroz LP, Pennington RT, De Lima HC, Fonty E, Wojciechowski MF, Lavin M (2012) Revisiting the phylogeny of Papilionoid legumes: New insights from comprehensively sampled early-branching lineages. American Journal of Botany 99(12): 19912013. https://doi.org/10.3732/ajb.1200380 
Cardoso D, Pennington RT, De Queiroz LP, Boatwright JS, Van Wyk B-E, Wojciechowski MF, Lavin M (2013) Reconstructing the deep-branching relationships of the Papilionoid legumes. South African Journal of Botany 89: 58-75. https://doi.org/10.1016/j. sajb.2013.05.001

Clark R (2008) Revision of the genus Sarcodum (Leguminosae: Papilionoideae: Millettieae). Kew Bulletin 63(1): 155-159. https://doi.org/10.1007/s12225-007-9003-9

Compton JA (2015a) A vignette of botany in the age of enlightenment: The story of Catesby's climber or the Carolina kidney-bean tree, Wisteria frutescens Leguminosae (Fabaceae). Botanical Magazine 32(3-4): 193-247. https://doi.org/10.1111/curt.12111

Compton JA (2015b) Wisteria sinensis on the slow boat from China: The journey of Wisteria to England. Botanical Magazine 32(3-4): 248-293. https://doi.org/10.1111/curt.12112

Compton JA (2015c) Wisteria japonica: Kaempfer's jama fuji. Botanical Magazine 32(3-4): 374-382. https://doi.org/10.1111/curt.12116

Compton JA (2015d) Wisteria brachybotrys Leguminosae (Fabaceae). Botanical Magazine 32(3-4): 294-311. https://doi.org/10.1111/curt.12113

Compton JA (2016) Typification of Wisteria megasperma F.Muell. (Fabaceae). Botanical Magazine 33(4): 374. https://doi.org/10.1111/curt.12116

Compton JA, Lack HW (2012) The discovery, naming and typification of Wisteria floribunda and W. brachybotrys (Fabaceae) with notes on associated names. Willdenowia 42(2): 219240. https://doi.org/10.3372/wi.42.42207

Compton JA, Thijsse G (2013) The type of Wisteria japonica (Fabaceae): Research into material for new names published in the first section of Siebold and Zuccarini's Flora Japonica. Willdenowia 43(1): 113-120. https://doi.org/10.3372/wi.43.43113

Compton JA, Thijsse G (2015) Wisteria floribunda with the tortuous tale of the Japanese wisteria with the longest racemes. Botanical Magazine 32(3-4): 312-356. https://doi. org/10.1111/curt.12114

Compton JA, Lane C (2019) Wisteria: The Complete Guide. Royal Horticultural Society, Peterborough.

Cooper W (2013) Australian rainforest fruits: a field guide. CSIRO publishing, Collingwood, Victoria. Australia. https://doi.org/10.1071/9780643107854

Craib WG (1927) Afgekia p. 376 in Contributions to the Flora of Siam, Additamentum XXIII. Bulletin of Miscellaneous Information, Kew 9: 374-394. https://doi.org/10.2307/4107547

Craib WG (1928) Florae Siamensis Enumeratio: a list of plants known from Siam, with records of their occurrence Vol. 1. Polypetalae. Siam Society, Bangkok, 395-398.

Dalzell NA, Gibson A (1861) Bombay Flora. Educations Society’s press, Byculla, Bombay.

Darriba D, Taboada GL, Doallo R, Posada D (2012) JModelTest 2: More models, new heuristics and parallel computing. Nature Methods 9(8): 772. https://doi.org/10.1038/nmeth.2109

Doyle JJ, Doyle JL (1987) A Rapid DNA Isolation Procedure for Small Quantities of Fresh Leaf Tissue. Phytochemical Bulletin 19(1): 11-15.

Doyle JJ, Doyle JL, Ballenger JA, Dickson EE, Kajita T, Ohashi H (1997) A phylogeny of the chloroplast gene $r b c \mathrm{~L}$ in the Leguminosae: Taxonomic correlations and insights into the evolution of nodulation. American Journal of Botany 84(4): 541-554. https://doi. org/10.2307/2446030 
Doyle JJ, Chappill JA, Bailey DC, Kajita T (2000) Towards a comprehensive phylogeny of legumes: evidence from $r b c \mathrm{~L}$ sequences and non-molecular data in: Herendeen PS, Bruneau A (Eds). Advances in Legume Systematics. Royal Botanic Gardens, Kew. London, 1-20.

Dunn ST (1911) Adinobotrys and Padbruggea in Bulletin of Miscellaneous Information, Kew 1911(4): 193-198. https://doi.org/10.2307/4119487

Dunn ST (1912a) A Revision of the Genus Millettia, Wight. \& Arn. Journal of the Linnean Society. Botany. London 41(280): 123-243. https://doi.org/10.1111/j.1095-8339.1912. tb02482.x

Dunn ST (1912b) Adinobotrys or Whitfordiodendron in Bulletin of Miscellaneous Information, Kew 1912(8): 363-365. https://doi.org/10.2307/4111791

Dunning LT, Savolainen V (2010) Broad-scale amplification of matK for DNA barcoding of plants, a technical note. Botanical Journal of the Linnean Society. London 164: 1-9. https://doi.org/10.1111/j.1095-8339.2010.01071.x

Edgar RC (2004) MUSCLE: Multiple sequence alignment with high accuracy and high throughput. Nucleic Acids Research 32(5): 1792-1797. https://doi.org/10.1093/nar/gkh340

Elmer ADE (1910) Whitfordia and Whitfordiodendron, Leaflets of Philippine Botany 2: 689, 743.

Endlicher SL (1833) Prodromus Florae Norfolkicae sive Catalogus Stirpium quae in Insula Norfolk annis 1804 et 1805 a Ferdinando Bauer Collectae et Depictae. Vindobonae apud Fridericum Beck Universitatis Bibliopolam. https://doi.org/10.5962/bhl.title.6703

Endlicher SL (1843) Callerya in Genera plantarum Supplementum 3. Apud: Fridericum Beck, Vindobonae [Vienna].

Fay M, Swensen SM, Chase MW (1997) Taxonomic affinities of Medusagyne oppositifolia (Medusagynaceae). Kew Bulletin 52(1): 111-120. https://doi.org/10.2307/4117844

Fay M, Bayer C, Alverson WS, de Bruijn AY, Chase MW (1998) Plastid rbcL sequence data indicate a close affinity between Digodendron and Bixa. Taxon 47(1): 43-50. https://doi. org/10.2307/1224017

Geesink R (1984) Scala Milletiarum, a survey of the genera of the Millettieae (Legum. -Pap.) with methodological considerations. Leiden Botanical Series 8.

Gray A (1858) Diagnostic characters of new species of phanogamous plants collected in Japan by Charles Wright, botanist of the U. S. North Pacific Exploring Expedition. (published by request of Captain John Rodgers, Commander of the Expedition). With observations upon the relations of the Japanese Flora to that of North America, and of other parts of the northern temperate zone. Memoirs of the American Academy of Arts and Sciences n.s. 6(2): 386. https://doi.org/10.2307/25057953

Harden GJ (2000) Callerya in Flora of New South Wales vol. 2. New South Wales University Press, Sydney. 615.

Hasskarl JK (1842) Marquartia globosa in Plantarum genera et species novae aut reformatae javenses. Literaturberichte zur Flora 25 band 2(2): 14.

Hu J-M, Chang S-P (2003) Two new members of the Callerya Group (Fabaceae) based on phylogenetic analysis of $r b c \mathrm{~L}$ sequences: Endosamara racemosa (Roxb.) Geesink and Callerya vasta (Kosterm.) Schot. Taiwania 48(2): 118-128.

Hu J-M, Lavin M, Wojciechowski MF, Sanderson MJ (2000) Phylogenetic systematics of the tribe Millettieae (Leguminosae) based on chloroplast trnK/matK sequences and its impli- 
cations for evolutionary patterns in Papilionoideae. American Journal of Botany 87(3): 418-430. https://doi.org/10.2307/2656638

Hu J-M, Lavin M, Wojciechowski MF, Sanderson MJ (2002) Phylogenetic analysis of nuclear ribosomal ITS/5.8S sequences in the tribe Millettieae (Fabaceae): Poecilanthe-Cyclobium, the core Millettieae, and the Callerya Group. Systematic Botany 27(4): 722-733.

Iwatsuki K, Boufford DE, Ohba H (2001) Flora of Japan Vol. 2b, Angiospermae, Dicotyledonae, Archychlamydeae (b). Tokyo: Kodansha.

Jansen RK, Wojciechowski MF, Sanniyasi E, Lee S-B, Daniell H (2008) Complete plastid genome sequence of the chickpea (Cicer arietinum) and the phylogenetic distribution of rps12 and $c l p \mathrm{P}$ intron losses among legumes (Leguminosae). Molecular Phylogenetics and Evolution 48(3): 1204-1217. https://doi.org/10.1016/j.ympev.2008.06.013

Kajita T, Ohashi H, Tateishi Y, Bailey CD, Doyle JJ (2001) rbcL and Legume Phylogeny, with Particular Reference to Phaseoleae, Millettieae, and Allies. Systematic Botany 26(3): 515-536.

Kearse M, Moir R, Wilson A, Stones-Havas S, Cheung M, Sturrock S, Buxton S, Cooper A, Markowitz S, Duran C, Thierer T, Ashton B, Meintjes P, Drummond A (2012) Geneious Basic: An integrated and extendable desktop software platform for the organization and analysis of sequence data. Bioinformatics (Oxford, England) 28(12): 1647-1649. https:// doi.org/10.1093/bioinformatics/bts199

Kim N-R, Kim Y-I, Lee J-H, Kim Y-D (2013) Genetic diversity of Millettia japonica in Korea as revealed by ISSR analysis. Korean Journal of Plant Taxonomy 43(4): 267-273. https:// doi.org/10.11110/kjpt.2013.43.4.267

Kirkbride JH, Gunn CR, Weitzman AL (2003) Fruits and seeds of Genera in the Subfamily Faboideae (Fabaceae) vol. 1. USDA. Technical Bulletin 1890.

Klitgaard BB, Lavin M (2005) Tribe Dalbergieae. In: Lewis G, Schrire B, Mackinder B, Lock M (Eds) Legumes of the World. Royal Botanic Gardens, Kew, 307-335.

Koller B, Delius H (1980) Vicia faba chloroplast DNA has only one set of Ribosomal RNA genes as shown by Partial Denaturation Mapping and R-Loop Analysis. Molecular \& General Genetics 178(2): 261-269. https://doi.org/10.1007/BF00270471

Lackey JA (1981) Tribe 10. Phaseoleae DC. (1825). In: Polhill RM, Raven PH (Eds) Advances in Legume Systematics, Part 1. Royal Botanic Gardens, Kew, 301-327.

Larsson A (2014) AliView: A fast and lightweight alignment viewer and editor for large data sets. Bioinformatics (Oxford, England) 30(22): 3276-3278. https://doi.org/10.1093/bioinformatics/btu531

Lavin M, Schrire B (2005) Tribe Sesbanieae. In: Legumes of the World, Lewis G, Schrire B, Mackinder B, Lock M (Eds) Royal Botanic Gardens, Kew, 452-453.

Lavin M, Doyle JJ, Palmer JD (1990) Evolutionary significance of the loss of the ChloroplastDNA Inverted Repeat in the Leguminosae subfamily Papilionoideae. Evolution (Lancaster) 44(2): 390-402. https://doi.org/10.1111/j.1558-5646.1990.tb05207.x

Lavin M, Eshbaugh E, Hu J, Mathews S, Sharrock RA (1998) Monophyletic subgroups of the tribe Millettieae (Leguminosae) as revealed by phytochrome nucleotide sequence data. American Journal of Botany 85(3): 412-433. https://doi.org/10.2307/2446334

Lavin M, Herendeen PS, Wojciechowski MF (2005) Evolutionary rates analysis of Leguminosae implicates a rapid diversification of lineages during the Tertiary. Systematic Biology 54(4): 530-549. https://doi.org/10.1080/10635150590947131 
Lewis G (2012) Callerya cinerea Leguminosae in Curtis's Botanical Magazine n.s. 29(2): 137143 t. 732. https://doi.org/10.1111/j.1467-8748.2012.01779.x

Lewis G, Schrire B, Mackinder B, Lock M (Eds.) (2005) Legumes of the World. Royal Botanic Gardens, Kew, UK.

Li J, Jiang J-H, Fu C-X, Tang S-Q (2014) Molecular systematics and biogeography of Wisteria inferred from nucleotide sequences of nuclear and plastid genes. Journal of Systematics and Evolution 52(1): 40-50. https://doi.org/10.1111/jse.12061

Liston A (1995) Use of the polymerase chain reaction to survey for the loss of the inverted repeat in the legume chloroplast genome. In: Crisp MD, Doyle JJ (Eds) Advances in legume systematics, part 7, phylogeny. Royal Botanic Gardens, Kew, Richmond, Surrey, 31-40.

Lôc PK (1996) Callerya Endl. (Leguminosae-Papilionoideae-Millettieae) in the Flora of Vietnam, Cambodia and Laos. Vietnam Journal of Science and Technology 2: 53-64.

Lôc PK, Vidal JE (2001) In: Morat P (Ed.) Flore du Cambodge, du Laos et du Viet-Nam vol. 30, Leguminosae - Papilionoideae - Millettieae. Muséum National d'Histoire naturelle, Paris, 1-191.

LPWG (2013) Legume phylogeny and classification in the $21^{\text {st }}$ century: Progress, prospects and lessons for other species-rich clades. Bruneau A, Doyle JJ, Herendeen P, Hughes C, Kenicer G, Lewis G, Mackinder B, Pennington RT, Sanderson MJ, Wojciechowski MF. Taxon 62(2): 217-248. https://doi.org/10.12705/622.8

Mattapha S (2017) PhD Thesis. The Systematics of Tribe Millettieae (Leguminosae-Papilionoideae). School of Biological Sciences, The University of Reading, UK, 1-209.

Merrill ED (1934) New Sumatran Plants 1. Papers of the Michigan Academy of Science 19(Part 1): 149-203.

Miquel FAW (1855) Flora van Nederlandsch Indie 1(1). Tribus Millettieae. C.G. van der Post, Amsterdam; C. van der Post, Utrecht; Friedrich Fleischer, Leipzig, 137-158.

Mueller F (1858) Fragmenta Phytographiae Australiae vol. 1. Government of Victoria, Melbourne. https://doi.org/10.5962/bhl.title.116758

Murrill WA (1905) Amauroderma Murrill in Polyporaceae of North America. Bulletin of the Torrey Botanical Club 32: 366. https://doi.org/10.2307/2478510

Murrill WA (1908) Whitfordia Murrill in Additional Philippine Polyporaceae. Bulletin of the Torrey Botanical Club 35(8): 407. https://doi.org/10.2307/2479285

Noltie HJ (2002) The Dapuri Drawings, Alexander Gibson and the Bombay Botanic Gardens. Antique Collectors Club with Royal Botanic Garden, Edinburgh.

Noltie HJ (2016) The Cleghorn Collection South Indian Botanical Drawings 1845 to 1860. Royal Botanic Garden, Edinburgh.

Nuttall T (1818) Wisteria Nutt in Genera of North American plants 2. D. Heartt, Philadelphia.

Ohwi J (1984) Millettia. In: Ohwi J, Meyer FG, Walker EH (Eds) Flora of Japan (reprinted revised edition). Washington D.C. Smithsonian Institution, 572.

Palmer JD, Thompson WF (1981) Rearrangements in the chloroplast DNA of mung bean and pea. Proceedings of the National Academy of Sciences of the United States of America 78(9): 5533-5537. https://doi.org/10.1073/pnas.78.9.5533

Palmer JD, Osorio B, Aldrich J, Thompson WF (1987) Chloroplast DNA evolution among legumes: Loss of a large inverted repeat occurred prior to other sequence rearrangements. Current Genetics 11(4): 275-286. https://doi.org/10.1007/BF00355401 
Panigrahi G, Murti SK (1989) Flora of Bilaspur District, Madhya Pradesh vol. 1. Botanical Survey of India.

Prathepha P (1994) Chromosome number of the genus Afgekia Craib. Cytologia 59(4): 437438. https://doi.org/10.1508/cytologia.59.437

Prathepha P, Baimai V (2003) Molecular Characterization of the Divergence of Rare Species of the Genus Afgekia (Papilionoideae, Tribe Tephrosieae) by RAPD Markers and Nucleotide Sequence Analysis. ScienceAsia 29(1): 13-20. https://doi.org/10.2306/scienceasia1513-1874.2003.29.013

de Quieroz LP, Pastore JFB, Cardoso D, Snak C, de Lima HC, Gagnon E, Vatanparast M, Holland AE, Egan AN (2015) A multilocus phylogenetic analysis reveals the monophyly of a recircumscibed tribe Diocleae with well-supported generic relationships. Molecular Phylogenetics and Evolution 90: 1-19. https://doi.org/10.1016/j.ympev.2015.04.016

Rafinesque CS (1817) Diplonix elegans Raf., Florula Ludoviciana or a Flora of the State of Louisiana. C. Wiley and Co. New York. 101-102.

Rambaut A, Drummond AJ (2009) Tracer 1.5. http://beast.bio.ed.ac.uk/Tracer

Reveal JL (1997) Typification of Glycine frutescens. In: Turland N, Jarvis C (Eds) Typification of Linnaea specific and varietal names in the Leguminosae (Fabaceae). Taxon 46(3): 457-485. https://doi.org/10.2307/1224388

Robinson BL, Fernald ML (1908) Gray's New Manual of Botany. 7th edition. American Book Company, New York, Cincinnatti, Chicago. 515.

Ronquist F, Huelsenbeck JP (2003) MrBayes 3: Bayesian phylogenetic inference under mixed models. Bioinformatics (Oxford, England) 19(12): 1572-1574. https://doi.org/10.1093/ bioinformatics/btg180

Roxburgh W (1814) Hortus Bengalensis. Mission Press, Serampore, India.

Roxburgh W (1832) Robinia racemosa in Flora Indica ed. 1832. Carey W (Ed) vol. 3. Serampore: W. Thacker \& Co. Calcutta; Parbury, Allen \& Co. London, 329.

Samarakoon T, Wang SY, Alford MH (2013) Enhancing PCR amplification of DNA from recalcitrant plant specimens using a trehalose-based additive. Applications in Plant Sciences 1(1): 1200236. Published online2013Jan. https://doi.org/10.3732/apps.1200236

Särkinen T, Staats M, Richardson JE, Cowan RS, Bakker FT (2012) How to Open the Treasure Chest? Optimising DNA Extraction from Herbarium Specimens. PLoS One 7(8): e43808. https://doi.org/10.1371/journal.pone.0043808

Schot AM (1994) A Revision of Callerya Endl. (including Padbruggea and Whitfordiodendron) (Papilionaceae: Millettieae). Blumea 39: 1-40.

Schrire BD (2005) Tribe Millettieae. In: Legumes of the World, Lewis G, Schrire BD, Mackinder B, Lock M (Eds) Royal Botanic Gardens, Kew, 367-387.

Schrire BD, Lavin M, Barker NP, Forest F (2009) Phylogeny of the tribe Indigofereae (Leguminosae - Papilionoideae): Geographically structured more in succulent-rich and temperate settings than in grass-rich environments. American Journal of Botany 96(4): 816-852. https://doi.org/10.3732/ajb.0800185

Shaw J, Lickey EB, Schilling EE, Small RL (2007) Comparison of whole chloroplast genome sequences to choose non-coding regions for phylogenetic studies in angiosperms: The tortoise and the hare III. American Journal of Botany 94(3): 275-288. https://doi. org/10.3732/ajb.94.3.275 
Siebold PFB, Zuccarini JG (1839) Wisteria in Flora Japonica vol. 1 (parts 9-10). Lugduni Batavorum [Leiden]: apud auctorem.

Sirichamorn Y (2006) MSc thesis. Morphometrics and Molecular Systematics of the Genus Afgekia Craib (Fabaceae). Department of Botany, Faculty of Science, Chulalongkorn University, Thailand.

Sirichamorn Y, Balslev H, Mattapha S (2016) Two new species of Callerya Endl. (Leguminosae: Papilionoideae) from Thailand. Phytotaxa 263(1): 42-50. https://doi.org/10.11646/ phytotaxa.263.1.4

Soga K, Teruya F, Tateno H, Hirabayashi J, Yamamoto K (2013) Terminal N-Acetylgalactosamine-Specific Leguminous Lectin from Wisteria japonica as a Probe for Human Lung Squamous Cell Carcinoma. PLoS One 8(12): e83886. https://doi.org/10.1371/journal. pone.0083886

Soltis PS, Soltis DE, Smiley CJ (1992) An rbcL sequence from a Miocene Taxodium. Proceedings of the National Academy of Sciences of the United States of America 89(1): 449-451. https://doi.org/10.1073/pnas.89.1.449

Sprague TA (1904) New or Noteworthy Plants Wisteria involuta (sp. nov.) Gardeners'. Chronicle ser.3 36: 141.

Sprague TA (1905) New or Noteworthy Plants Wisteria involuta = Derris involuta Gardeners'. Chronicle ser.3 38: 3.

Stamatakis A (2014) RAxML version 8: A tool for phylogenetic analysis and post-analysis of large phylogenies. Bioinformatics (Oxford, England) 30(9): 1312-1313. https://doi. org/10.1093/bioinformatics/btu033

Sun H, Pedley L (2010) Sarcodum scandens in Flora of China Illustrations Fabaceae, Wu Zhengyi, Raven PH and Hong Deyuan (Eds) Science Press, Beijing, China, Missouri Botanical Garden Press, USA. 202.

Sun Y, Skinner DZ, Liang GH, Hulbert SH (1994) Phylogenetic analysis of Sorghum and related taxa using internal transcribed spacers of nuclear ribosomal DNA. Theoretical and Applied Genetics 89(1): 26-32. https://doi.org/10.1007/BF00226978

Taberlet P, Gielly L, Pautou G, Bouvet J (1991) Universal primers for amplification of three non-coding regions of chloroplast DNA. Plant Molecular Biology 17(5): 1105-1109. https://doi.org/10.1007/BF00037152

Thiers B (2019) Index Herbariorum: A Global Directory of Public Herbaria and Associated Staff. New York Botanical Garden's Virtual Herbarium.

Turland NJ, Wiersema JH, Barrie FR, Greuter W, Hawksworth DL, Herendeen PS, Knapp S, Kusber W-H, Li D-Z, Marhold K, May TW, McNeill J, Monro AM, Prado J, Price MJ, Smith GF (Eds.) (2018) International Code of Nomenclature for algae, fungi, and plants (Shenzhen Code) adopted by the Nineteenth International Botanical Congress Shenzhen, China, July 2017. Regnum Vegetabile 159. Glashütten: Koeltz Botanical Books. https://doi.org/10.12705/Code.2018 Valder P (1995) Wisterias - a comprehensive guide. Timber Press, Portland, Oregon. USA.

Vogel JRT (1843) Marquartia in F.J.F.Meyen's beitrage zur botanik, gesammelt auf einer reise um die erde. Novorum Actorum Academiae Caesareae Leopoldinae-Carolinae Naturae Curiosorum 19. Supplementum 1.

Wallich N (1830) Pongamia atropurpurea in Plantae Asiaticae Rariores or descriptions and Figures of a Select Number of Unpublished East Indian Plants vol. 1. Treutel and Würtz, 
Treutel jun. and Richter, London, Treutel and Würtz, Strasburgh and Paris. 70. https://doi. org/10.5962/bhl.title.468

Wei Z (1985) A Revision of the Chinese Millettia (Papilionoideae) (cont.). Zhiwu Fenlei Xuebao 23: 27-292.

Wei Z, Pedley L (2010) Callerya. In: Wu Z-Y, Raven PH, Hong DY (Eds) Flora of China 10.

Science Press, Beijing; Missouri Botanical Garden Press, St Louis, 181-187.

Wei Z, Chen D, Zhang D, Sun H, Pedley L (2010) Tribe Millettieae. In: Wu Z-Y, Raven PH, Hong DY (Eds) Flora of China 10 Fabaceae. Science Press, Beijing; Missouri Botanical Garden Press, St Louis, 165-196.

White TJ, Bruns TD, Lee SB, Taylor JW (1990) Amplification and direct sequencing of fungal ribosomal RNA Genes for phylogenetics. PCR - Protocols and Applications - A Laboratory Manual. Academic Press, 315-322. https://doi.org/10.1016/B978-0-12-372180-8.50042-1 Wight R, Arnott GA (1834) Millettia in Prodromus florae peninsulae Indiae orientalis vol. 1. Parbury, Allen \& Co. London, 263.

Wink M (2013) Evolution of secondary metabolites in legumes (Fabaceae). South African Journal of Botany 89: 164-175. https://doi.org/10.1016/j.sajb.2013.06.006

Wojciechowski MF (2013) Towards a new classification of Leguminosae: Naming clades using non-Linnaean phylogenetic nomenclature. South African Journal of Botany 89: 85-93. https://doi.org/10.1016/j.sajb.2013.06.017

Wojciechowski MF, Sanderson MJ, Steele KP, Liston A (2000) Molecular phylogeny of the "temperate herbaceous tribes" of papilionoid legumes: a supertree approach in Advances. In: legume systematics Herendeen PS, Bruneau A (Eds) Royal Botanic Gardens, Kew, 277-298. Wojciechowski MF, Lavin M, Sanderson MJ (2004) A phylogeny of legumes (Leguminosae) based on analysis of the plastid matK gene resolves many well-supported subclades within the family. American Journal of Botany 91(11): 1846-1862. https://doi.org/10.3732/ ajb.91.11.1846

Zhu X-Y (1994) Wisterieae, a new Tribe of the family Leguminosae - with special reference to its pollen morphology. Cathaya 6: 115-124.

\section{Appendix I}

List of Herbarium specimens of newly circumscribed taxa examined for this study. All herbarium specimens seen for species transferred to newly described genera in this paper are documented as well as all vouchers used to illustrate Figure 2, distinctive morphological characters in Tribe Wisterieae.

\section{Austrocallerya australis}

AUSTRALIA: New South Wales: Cooper Creek Rd, north of Mullimbimby 8 November 1967, A.R.H.Martin 1392 (K). Norfolk island: upper slopes of Mt. Pitt, 26 August 1964 G. Uhe 1182 (K); Longridge distr. c. 400 ft., May-June 1937, Capt. J.D.McCornish 16 (K); in Hooker Herbarium 1867, Cunningham s.n. s.d.; south slope of Mt. Pitt elev. 600 ft, 16 December 1959, R.D.Hoogland 6640, (L1978550). 
Queensland: Moreton, Numinbah forest reserve, Nerang river, opposite Chesters Rd. 28 July 2012, P.I.Forster PIF38831 (US02324718).

PAPUA NEW GUINEA: Nondugl western highlands distr. c. $5000 \mathrm{ft}$. no date, J.S. Womersley NGF4470 (K, L1978542); 5 miles east of Okapa eastern highlands, 5000 ft., 24 September 1964, Thomas G.Hartley 13092 (K, L1978540); Tagalinga, Jimmi Valley, western highlands elev. $5100 \mathrm{ft} ., 16$ June 1955, J. Womersley \& A.Millar NGF7700 (K, L1978543); eastern highlands distr. track for Arau-Obura elev. 1500m. 18 October 1959, L.J.Brass 32129 (K, L1978545); 1 mile west of Kopiago airfiled, western highlands distr. elev. 4000 ft., 3 November 1968, J.S. Womersley, J.Vandenberg \& M.Galore NGF37315 (L1978545); western highlands, Komun-Pin divide, east of Korn, upper Wahgi valley, elev. 1650 m. 10 September 1956, R.D.Hoogland \& R.Pullen 6176, (L1978547, US02324718); eastern highlands distr. Arau to Obura, elev. 1500 m. 18 October 1959, L.J.Brass 32129 (K, L1978544); western highlands near Enjumanda village, middle Tale valley, Wabag distr. elev. 7000 ft., 27 June 1960, R.D.Hoogland \& R.Schodde 6773 (L1978541); Lower Fly river east bank opposite Sturt Island, October 1936, L.J.Brass 8214 (K, L1978546). Bougainville island: south slopes of Crown Prince Range elev. 2000 ft. 1936, A.H.Voyce D13 (K).

\section{Austrocallerya megasperma}

AUSTRALIA: New South Wales: Korumbyn Creek 8 miles southwest of Murwillumbah, 6 September 1972, R. Coveny \& A.N.Rodd 4516 (K, L1978814); Richmond river, received May 1867, C.Moore s.n., (K000880987); 25 km north of Lismore near Whian Whian, 30 October 1981 A.Kanis 2113 (CANB301997, L1978815). Queensland: Currumbin Creek road 28 September 1965, J.Gillieatt 399 (K); Moreton Bay district, s. d. 1859, W.Hill s.n. (K); Moreton Bay, 12 October 1958, D.Norris s.n. (K); Fraser Island, 15-16 October 1930, C.E.Hubbard 4422 (K, L1978813); Kunda Park on the Marrochydore Rd, 11 May 1977, J.A.Elsol 121 (K); eastern spur of McPherson Range 47 miles south of Ipswich, 5 April 1953, R. Melville \& T.Hunt 3634 (K); Como State Forest, Kooloolah, Gympie, 13 October 1988, P.L.Swanborn QL883, (L1978812); Moreton sports Rd. Bli Bli, 23 July 2012, G.Leiper, AQ818814 (US02324711).

\section{Austrocallerya pilipes}

AUSTRALIA: Queensland: Cape York, Harvey Creek elev. 30 m. rainforest, 23 October 1989, B.Gray 20267 (K, L3884382); Cape York, State Forest Rd 143, Parish of Riflemead, Lerra Logging Area, elev. 500 m. 25 October 1989, B. Gray 05144 (K); SFR Riflemead, Lerra LA, elev. 500 m. 3 July 1986, B. Gray 04932 (K); SFR 143 Lerra LA elev. 1200 m. 27 October 1988 B.Gray 04932 (K); Johnstone river, August 1892, F.M.Bailey s.n., (K000880982); Cook Co., start of Bartle Frere walking track, 5 December 2001, R.Booth \& R.Jensen 2788, (L3892264, NY03556966); rain forest Mt Lewis road $5 \mathrm{~km}$ from main road, 5 November 2002, B. Gray 08360, (L3894665); North Queensland, Mossman river gorge, 3 February 1959, L.J.Brass 2133 (US02324776). 


\section{Kanburia chlorantha}

THAILAND: Tak Prov.: N. Tak Lansang, Pa Ban Om Yom, elev. 520 m., 9 August 1968, S. Phengnaren, (BKF57659, L1978942, L1978943).

\section{Kanburia tenasserimensis}

THAILAND: Kanburi Prov.: Ta Salao, Kanburi, 10 July 1930, Phraisurind Put 3049; Ta Salao 10 July, 1930, Put 3049 (K, L1978938, L0475175).

\section{Nanhaia fordii}

CHINA: Guangxi Prov.: Zhuang Autonomous Region, Pingle Co. Guangyun forest farm, Maozi Chong, 9 October 1958, Yinkun Li 401972, (IBK00073457); Guangxi, Zhuang Autonomous Region, Guixian Longyang distr. Tanyang township, 29 June 1957, Chen Zhaozhou 50790, (IBK00073456).

VIETNAM: Taai Wong Mo Shan, vicinity of Tong Fa, Ha-Coi, Tonkin, 11-23 September 1939, W.T.Tsang 29588, (P02753519).

\section{Nanhaia speciosa}

CHINA: Guandong Prov.: Thai-Yong, elev. $2000 \mathrm{ft}$. , west of Swatow [Shantou], Dr. J.M.Dalziel s.n., (E00124678); Tai-O [Lantau island], 17 August 1929, Tsiang Ying 3105, (E00124674); King P'ing Shan, T'sang Faan, Feng Ch'eng distr. 1-9 September 1936, W.T.Tsang 26766 (K); Hong Kong, Sai Tsui, New Territories opposite High Island, 21 October 1969, Shiu Ying Hu 8313 (K); Hong Kong, Herb. of U.S. North Pacific Exploration Exped. Rinngold \& Rodgers 1853-1856, C. Wright 138 (K); Hong Kong, Pine Grove New territories, 21 September 1969, Shiu Ying Hu 7924 (K); Hong Kong, Ma On Shan, Sai Kung, 20 August 1973, Kit Yock Chan 125, (P02754272); Hong Kong, Pat sin ling N. T. 29 October 1968, Shiu Ying Hu 6091 (K). Hainan Island: Liamui, elev. 400 m, June-July 1935, J.Linsley Gressitt 1194, (E00124679); Lingnan Univ. 17405, Paak Shak shan, Ngau Ku Tsai Lak, 12 June 1928, Wait-Tak Tsang 656 (K); Dung-ka elev. 1700 ft. 1932-1933, N.K.Chun \& C.L.Tso 43467 (K); Ngau Tsai T'ang, Nodoa, Taam-chau distr., 18 July 1927, W.T.Tsang 66 (K); Ngou Mou Tai Lik, Pak Shik Ling, Ku Tung village, Ching Mai distr., 27 September 1932, C.I.Lei 31 (K).

VIETNAM: Ouonbi, Tonkin, October 1885, B.Balansa 1205 (K); environs de porte de Bat Bae, September 1888, B.Balansa 2230 (K, P02754259, P02754261); Sai Wong Mo Shan (Sai Vong Mo Leng) Lomg Ngong village, Dam-Ha, 18 July-9 September 1940, W.T.Tsang 30375 (K, P02754251, P02754254); Tsai Wong Mo Shan, Tong Fa market, Ha-coi, 11-23 September 1939, W.T.Tsang 29510 (K, P02754253): Tsai Wong Mo Shan, Tong Fa market, Ha-coi, 11-23 September 1939, W.T.Tsang 29542 (K, P02754252); Tsai Wong Mo Shan,Chuk-phai, Ha-coi, 18 November - 2 December 1936, W.T.Tsang 27192 (K, P02754255); Phu Ho, August 1923, Petelot 1088 (K, P02754256, P02754257); Kau 
Nga Shan, Tien-yen, 1-9 January 1937, W.T.Tsang 27534 (K, P02754258); Ninh Thai, Muou Lang 14 September 1888, H.Bon 3931 (K, P02754260, P02754262, P02754263); Ninh Thai, 21 August 1889, H.Bon s.n. (K, P02754264); Guang You, Tonkin, August 1885, B.Balansa 1204 (K, P02754265, P02754274); Ouonbi, Tonkin, 13 September 1885, B.Balansa 1203 (K, P02754266, P02754267, P02754268).

\section{Serawaia strobilifera}

INDONESIA: [Borneo] Kalimantan: east Kutei, Susuk region, elev. 10 m, 26 June 1951, A.Kostermans 5442 (K, L1978967); Kalimantan east, Kutei, Sangkulirang [Miang] island elev. 30 m, 24 May 1951, A.Kostermans 4898 (K, L1978985); Kalimantan, west Kutei, near Long Liah Leng elev. 250 m, 31 August 1925, F.H.Endert 3031 (K, L1978972); west Kutei elev. 130 m, 18 August 1925, F.H.Endert \& L.Iboet 24 (K); Kalimantan central, Bukit Raya Exped. 21 November 1982, upper Katingan (Mendawai) river area, upper Samba river Tumbang Habangoi to Tumbang Riang, elev. 50 m., J.P.Mogea \& W.J.J.O de Wilde 3547 (L); west Koetei, elev. 50 m, exped. Midden-oost Borneo, 7 August 1925, F.H.Endert 2387 (L1978976, L1978973); Bukit Raya Exped. Tumbang Atey, elev. 100 m, 28 January 1983, Wiriadinata 3444 (L0475064, L1978974); West Koetai no. 24, steep river bank, elev. 130 m, 18 August 1925, F.H.Endert \& L.Iboet 2653 (L0503365); Kalimantan east, Gunung Kongkat to Gunung Kongbotak, elev. 350 m, 27 January 1981, Masahiro Kato and Harry Wiriadinata B-5145 (L1978978); east Kutei, G. Tepian Lobang, on Menubar river north-east of Sangkulirang, elev. 200 m, 7 August 1951, A.Kostermans 6036 (L1978983).

MALAYSIA: [Borneo] Sabah: Ranau distr., Kampung Takutan, Tapi Sungai mekeden, Tengkunanawau, 15 May 1995, Lomudin Tadong 308 (K); Tawau, Kawa rd., 30 May 1962, Jaswir \& Aban SAN 26274 (K, L1978984); Sungai [river] Raoen, 1893-1894, H.Hallier 3364 (K, L1978968); Diwata, Kennedy Bay [timber] Co., 9 June 1961, F.R.Muin Chai SAN 25083 (K); Segaluid Lokan Forest Reserve, Sandakan, 25 January 1975, Aban \& Leopold SAN 80986 (K); mile 12 Hap Seng timber complex, Brantian rd., climber 28 ft., 17 March 1979, Fidilis Krispinus SAN 89763 (K, L1978970); Kabili Sepilok Forest Reserve, Elopura Forest Distr., climber 25 ft., 4 May 1939, Enggoh 10531 (K, L1978969); Lahad Datu, mile 4, Silam, elev. 500 ft., 22 July 1966, Ahmad Talip 54923 (L1978975); Sabah? Bukit Batai, Exped, Nieuwenhuis 1896-1897, July 1897, Jaheri 374 (K, L0503363); Pinangah forest reserve, Telupid distr. 12 December 1993, Fidilis Krispinus SAN 135655 (L1978981).

\section{Sigmoidala kityana}

THAILAND: Chiangmai Prov.: elev. c. 300 m, 8 September 1921, A.F.G.Kerr 5658 (K, BK258012); Siam, Me-Ban-Prae, 11 October 1929, C.W.Franck 599 (L1978811); Loei, Nong Hin, Ban Suan Hom village, Suan Sawan karst limestone, 15 November 2011, R.P.Clark 245 with P.Wilkin, P.Suksathan, K.Keeratikiat, A. Trias-Blasi \& Mr Phitak(K); Lampun, Me Li, elev. 270 m. 13 November 1925, Winit 1557 (K, BK258013). 


\section{Wisteriopsis championii}

CHINA: Fujian Prov.: March 1962, Cai Jue s.n., (NAS00390280); (as Fukien) Foochow, Ja-Miao shan, no date, H.H.Chung 2786 (K); (as Fukien), Yuping, Huang-chin shan, elev. 130 m. 17 August 1924, H.H.Chung 3008 (K). Guandong Prov.: Haifeng County, rare beside road, 20 August 1958, Wei Zhaofen 121335 (PE00410165); Hong Kong, FoTan south valley, fls greenish-white, leaves glabrous, stipuleoles setose, 20 June 1970, Shiu Ying Hu 10476 (K, PE00410166); Ruyuan Yao Autonomous Co., mountain habitat between Linxia and Baishui villages, 14 August 1935, W.Y.Chun [Chen Huanyu] 10776 (PE00410167); Ying Tak [Yingde Distr.], Wen Tong Shan [Wentangshan], 6 June 1932, T.M.Tsui 412 (PE00410168); Dapu County, Tai Mo Shan in clay by roadside among thickets, 13 July 1932, W.T.Tsang 21166 (P02754297, PE00410169); Hong Kong, main island, on cliff beside shore, 27 September 1972, Shiu Ying Hu 12214 (K, PE00410170); Hong Kong, Cheung Shu Tan New Territory, flowers white, standard marked with a green spot at base, 19 May 1973, Kit Yock Chan 092 (P02753548, PE00410171); Hong Kong, Ma on Shan New Territories 22 September 1968, Shiu Ying Hu 5648 (PE00410172); Yingde Co. Wentangshan 6 June 1932, T.M.Tsui 412 (NAS00390863); Shixing Co. Yao township, 16 July 1958, Deng Liang 6906 (HITBC019545); Ruyuan Yao Autonomous Co. Baixia village among rocks 14 August 1935, Chen Huanyu 10776 (SZ00111312); C.Wright s.n. for Rinngold-Rodgers exped. 1853-1856 (K); Hong Kong, High Island, New Territories, 11 November 1969, Shiu Ying Hu 8626 (K); Hong Kong, June 1874 Herb. Hance 10121 (P02754293); Hong Kong, Mr [Robert] Fortune 62. 1845 Collection de la Societe Horticulturale de Londres 1856 (P02754294); Hong Kong, Mt. Gough, 22 May 1895, E.Bodinier 1209 (P02753547, P02754295); Hong Kong, C. Wright 136 for Rinngold and Rodgers exped 1853-1856 (P02754296).

\section{Wisteriopsis eurybotrya}

CHINA: Guangxi Prov:: Long-tcheou [Longzhou] received 14 February 1911, Dr. Simond s.n. (P02753456). Guizhou Prov.: (as Kouy-tcheou), Kiang Long, Keou Lau tse, August 1910, M.Cavalerie s.n. (P027534, P02753465).

VIETNAM: Hue and vicinity, Tourane, Annam, below Hue divide, May-July 1927, J. \& M.S.Clemens 3967 (P02753504, U1262152); in monte Chua Hae, 1 July 1889, H.Bon 2962 (P02651680); Tu Phap, August 1887, B.Balansa 2240 (P02753455); collines sur la rive gauche de la riviere noir, en face de Phuong Lam, 13 January 1887, B.Balansa 2302 (P02753444, P02753445); Kau Nga Shan and vicinity, Tien-yen, 1-9 January 1937, W.T.Tsang 27510 (P02753461); Canh Vrap, recd. December 1903, Dr Spire 1210 (P02753462); Day Bong Hong, gare de Cau Hai, Hue 14 September 1938, E.Poilane 27843 (P02753463); Annam, Col d'Ailao, Quang Tri Prov. 400 m. 1937, M.Poilane 26623 (P02753466); route de Phu Quy a Kebon, 4 August 1929, M.E.Poilane 16576 (P027534567); Ninh Binh Prov. Cuc Phuong Nat. Park, 26 August 1999, N.M.Cuong 463 (L1978805); Yen Lac village Yen Thuy distr. Hoa Binh Prov. 21 August 2000, N.M.Cuong, D.T.Kien \& M.V.Sinh 1021 (L1978804); Mt. Bani $25 \mathrm{~km}$ from Tourane, May-July 1927, J. \& M.S.Clemens 3742 (U1262153). 
LAOS: Expedition du Me-Kong, Paklai a Luang Prabang 1866-1868, Dr Thorel s.n. (P02753450, P02753458); Luang Prabang, 1890, Mussie s.n. for L.Pierre (P02753457); Luang Prabang 1866-1868, Dr Thorel 9441 (P02753459); Vientiane Prov. Hin Heup distr. Nam Lik river c. 5 km west of Khon Phol village elev. 225 m. 22 August 1999, J.F.Maxwell 99-145 (L1978803).

\section{Wisteriopsis japonica}

JAPAN: Honshu: precise location unknown, 24 Aug. 2003, M. Furusawa \& M. Miyake FOK-059278 (E00661878); Honshu cult. 12 Sept. 1937, T.Makino s.n. (L0282763); Yamamoto in Settsu 9 Sept. 1956, M.Togasi TNS [National Museum of Nature and Science] 1497 (K x 2); Hondo, Yamamoto, Kawabe-gun in Settsu, 3 June 1950, M. Togasi NSM 1. (L2010848, US02324943); Tarumi-ku, Koobe-shi, settu Prov. Pref. Hyogo. Mt. Mekko-yama, 22 Oct. 1975. Elev. 40-150m., Miyoshi Furuse 9745 (K); Kawanishi, Iwakuni city, Shiro-yama Nat. Forest, north bank of Misyo river, L.A.Charette 1834 (US02324044); Yamaguchi Pref. Kawanashi, Yokoyama village, Iwakuni city, Shiro-yama Nat. forest, north bank of Misyo river L.A.Charette 1557 (US02324045). Kyushu: US North Pacific Expedition 1853-1856, C. Wright s.n. (NY00016341); Nagasaki, 1863 ex herb. Horti bot. Petropolitani, Maximowicz s.n. Iter secundum (P02942301); Nagasaki, 1863 ex herb. Horti bot. Petropolitani, Maximowicz s.n. Iter secundum, (P02942305); Siebold s.n. Herb. Lugd.-Batav. 908.120-13 s.d., Siebold s.n. (L0176060); Herb. Lugd.-Batav. 908.120-13 s.d., Siebold s.n. (L0176061); Nagasaki, 1862, R.Oldham 386 (L2010846); ex Herbario Lugduno-Batavo s.d., H.Bürger s.n. (M0153889); ex Herbario Lugduno-Batavo s.d., H.Bürger s.n. (M0153890); ex Herbario Lugduno-Batavo s.d., H.Bürger s.n. (M0153891); Nagasaki. 1826, R. Oldham 386 (K x 2); Ssama-yama, Shinano, July 1883, Science Coll. Imp. Univ. [TI] collector unknown, s.n. (K); Moji [Kitakyushu] 19 Sept. 1906. Bushy hillside, S.T.Dunn 8526 (K); Nanokawa-tosa 22 July 1892, K. Watanabe s.n. (K); Nagasaki, 1892, Maximowicz s.n. (K); Nagasaki, July 1862, R. Oldham 801 (K); Miyazaki Pref., Koyu County. Uwae township, N.Eri 100555, s.d. (US02324041). Shikoku: Between Kaminokae and Umagura, Nakatosa town, Kochi Pref. 15 August 2003, Taku Miyazaki 308058 (L3892474); collector locality and date unknown "kofusi" Japan (L2010847); collector locality and date unknown "kofusi" Japan (L2010847); Shikoku without precise locality, 20 Nov. 1914, E.H.Wilson Arnold Arb. 7796 (K); C. Wright Rinngold \& Rodgers Exped. 1853-1856 without locality (K); Minoo, Higashidani Pref. Oosaka, 20 Aug. 1961, M. Togashi 7888 (K); Mie, Shima, Toba, collector and date unknown, (US02324042).

\section{Wisteriopsis kiangsiensis}

CHINA: Anhui Prov.: Lin yungchi, southern Anhwei elev. 250 m., 30 June 1925, climber 7 m. high, Qin Renchang [Ren-Chang Ching] 2881 ex Herbarium of Biological Laboratory Nanking China 5130 (PE00417692, para.!, K, isopara.!); Taiping Co. Zhangjiabang, Jiao village, by roadside 300 m. 18 June 1959, collector unknown Herb. number 0044 (NAS00390600); Taiping Co. Qidu commune, Longguang Brigade, 19 
June 1959, collector unknown 0814 (NAS00390591); Xiuning Co. among hills elev. 300 m. 17 June 1959 collector unknown 1973 (NAS00390609); Meiguang Co. Houtian Brigade, elev. 220 m. 1 July 1959, collector unknown 7032 (NAS00390597); Xiuning Co. near Wucheng, hillside forest, 27 June 1959, Danrenhua et al. 2627 (NAS00390608); Qimen Co. 11 October 1957, Deng Shubin et al. 4818 (NAS00390607); Anhwei, A.Rehder 3091, s.d. (PE00320076); Xiuning Co. five cities, 17 June 1959, collector unknown (PE00410855); Huangshan City, 18 June 1959, elev. 300 m. Chen Jiadong s.n. (PE00410856); Huangshan city, Taiping, 1959 collector unknown (PE00410857); Near Fenglong Temple elev. 120 m. Zhou, Xu, Pan \& Cheng 304 (PE00410858); Ye-hsien, southern Anhwei, 800ft climber to $15 \mathrm{ft}$. common, July 27 1925, Ren-Chang Ching Arnold Arb. 3091 (K); West Siunin, 1 August 1925, R.C.Ching 8835, (US). Guizhou Prov.: Jinping Co. riverside, 25 May 1964, Wang Yumin 0014 [as Millettia championii] (GFS0002828); Jinping Co. 20 September 1964, Wang Yumin 1154 [as Millettia championii] (GFS0002830); Jinping Co. 17 September 1964, Wang Yumin 0653 [as Millettia championii] (GFS0002829). Hunan Prov.: Linxiang Co. mountain habitat, 27 June 1974, collector unknown (PE00410872). Jiangxi Prov.: Wugongshan elev. 380 m., 21 September 1983, Zhao Qizheng \& Gao Xianming 1235 [as Millettia championii], (CSFI015674); Zixi Co. elev. 210 m. 3 November 2001, Shi Jianmin 011460 [as Millettia championii] (JXAU0007470); Guangchang elev. 490 m. 4 April 1965, Jiangxi Univ. 650552 collector unknown [as Millettia championii], (WUK0255610); Pengze, Yaofang Forest Farm, Taohongling Nature Reserve 18 July 2015, elev. 200 m., Li Enxiang, Liu Yizhen \& Cai Qiying 15070834, (JJF00019576); Xihong Reservoir, Taohongling 28 July 2006, Tan Chengming, Zhu Huanbing \& Wu Haiching 06804, (JJF00019575); Taihe Co. by river, elev. 170 m. 23 September 1973, Shi Xinghua 730132 [as Millettia championii] (JXAU0007475); Jinggangshan elev. 420 m. 28 July 1988, Sun Yegen 00495 [as Millettia championii] (JXAU0007474); Zixi Co. elev. 390 m. 30 October 2001, Liu Renlin et al. 011765 [as Millettia championii] (JXAU0007471); Taihe Co. by riverside elev. 170 m. 23 September 1973, Shi Xinghua 730132 [as Millettia championii] (JXAU0007476); Taohongling 18 July 2015 elev. 200-500 m. Li Enxiang et al. (JXU0007874, L2015070221); Xiushui Co. edge of bamboo forest elev. 500 m. 5 November 1995, Ye Cong [Ye Cunsu] 943 [as Millettia championii] (NAS00399073); Wuning County, Yishan, 31 July 1936, H.K. Teng 352 [as Millettia championii] (PE00410164); Longnan County, Cheng longxiang jiao keng, along streamside Moximu 21026, s.d., (PE00320077); Poyang Co. June 1959, Li Qihe \& Chen Ze 1014 (PE00410868); Jiangxi Prov. Dongxiang Co. hillside, 30 June 1959, Li Qibe \& Chen Ze 1434 (PE00410870); Wuning Co. 28 June 1947, Xiong Huiguo 05013 (PE00410869): Changkeng, 18 June 1936, H.K.Teng 229 (PE00410864); Changkeng, 18 June 1936, H.K.Teng 233 (PE00410863); Wuning Co. Yishan, 31 July 1936, H.K.Teng 353 (PE00410862); Wuning Co. Yishan, 8 August 1936 H.K.Teng 362 (PE00410866). Jiangsu Prov.: Qutang township by roadside elev. 142 m. 27 September 2015, Chen Gongxi, Zhang Daigui et al. LXP-06-5873, (JIU08535); Lianyungang, Huang wofujin, 14 Sept. 1952, Chen Changdu, Wang Jinting \& Dong Huimin 21023, (PE01566389). Zhejiang Prov.: Zhoushan, Jintang Island, Xishangang, 27 June 2012, Bi Yuke BYK1742, (CSH0055973); Jianye, Meishi Dashiping, 6 September 1979, Honglin s.n., (HZ020355); Yaofang Forest Farm, Taohongling Nature Reserve 18 
July 2015, elev. 200 m., Li Enxiang, Liu Yizhen \& Cai Qiying 15070834 (JXU0007861); Yanxia village forest, 11 November 2015, Tan Weizheng, Ding Qiaoling, Zhao Wanyi, Liu Zhongcheng, Zhang Zhong \& Ye Xi LXP-13-08399 (SYS00182930); near Hangzhou, 21 July 1980, Hang Zhibiao 1583, (NAS00396669); Yu-tsien Hsien, open thickets, 27 June 1927, Y.L.Keng 548 (PE00410859); Chunan Co. Tangcun farmhouse, Shangbao village 29 August 1980 collector unknown (PE00410860); Near Tatzechiao, 7 July 1915, F.N.Meyer 1517, (US).

\section{Wisteriopsis reticulata}

CHINA: Anhui Prov.: (as Anhwei) Ye-hsien elev. 50 ft. 3 July 1929, Ren-Chang Ching 3080 (K). Fujian Prov.: (as Fukien), 8 April 1923, H.H.Chung 1265 (K); (as Fukien), Minhow Hsien, Hwai-On, 17 July 1923, H.H.Chung 2464 (K); (as Fukien), Minhow Hsien, Pehling, 13 August 1923, H.H.Chung 2063 (K); (as Fukien), Kuliang Hills near Foochow elev. 1000-3000 ft, July-August 1919, J.B.Norton 1307 (K). Guandong Prov.: Hong Kong, Lantau Island, August 1886, collector unknown (K); Hong Kong, prope Tai Tam Tuk, June 1859, Hance 1489 (K); (as Kwangtung), Ying-tak Distr. 1 August 1921, Y.K. Wang 2914 (K); Guangdong, without locality, August 1887, flowers rose, C.Ford 36 (K); (as Canton) Theophilus Sampson s.n. 2 June 1885 (K); (as Kwangtung) August 1887, C.Ford 43 (K); (as Canton), 19 June 1886, Theophilus Sampson s.n. (K); (as Kwangtung) Wan Tong shan, Ying Tak Distr. 6-24 June 1932, T.H.Tsui 412 (K); (as Kwangtung), Lokcheng, 20 June 1929, C.L.Tso 21163 (K). Hainan Island: Chiu-shan, Ling-shui Co. Fan Haan Ts'uen, 4-20 May 1932, F.A.McClure 20117 (K); Santsigian (Lai), Chiu-san Tsuen (Ngai Distr.) 7-25 August 1932, S.K.Lau 369 (K); Hung-Mo shan, Sai In Low King Tang, 3 July 1929, Tsang \& Fung 422, Lingnan Univ. 17956 (K); Hainan, without precise locality 21 July 1938, C. Wang 33196 (K); Lin-Fa shan 8 August 1927, Tsang Wai-Tak 398, Lingnan Univ. 15397 (K); Lo King T’ang, Sin Wah (Twan Chan distr.) 18 May 1928, Tsang, Wai-Tak 354 Lingnan Univ. 17103 (K); Hainan, without precise locality, November 1889, A.Henry 8392 (K); Hainan, south of Fan Ta 19 April 1922, F.A.McClure 9168 (K). Hubei Prov.: Patung, October 1901, E.H.Wilson 1017 (K); Ichang, February 1887. A.Henry 2280 (K); Ichang, A.Henry 631 rec'd March 1886 (K); Western Hupeh, July 1907, E.H.Wilson 3282 (K);, Ichang, February 1887, A.Henry 1554A (K). Jiangsu Prov.: (as Kiangsu) summer 1926, C.L.Tso 1697 (K). Jiangxi Prov.: (as Kiangsi), Kinkiang, elev. 300 ft. 27 July 1909, E.H.Wilson 1648 (K); (as Kiangsi) Si Feng Sze, 12 August 1922, A.N.Steward 2648 (K). Taiwan: without location "Formosa" 1864 Richard Oldham 158 (K); (as Formosa) Kewkiang, Farinhoe, Dr Shrope s.n. comm. April 1875, Hance Herb. 1489 (K); Takeo-san (Apeo hills). 1912. W.R.Price 596 (K); Bankinsing, A.Henry 894 rec'd 1895 (K);), Kasenko to Santo, Kasenko Prov. 24 November 1918, E.H.Wilson 11099 (K). Zhejiang Prov.: Ningbo (as Ningpo) H.B.Forbes 2/76 coll. 1874 (K); (as Chekiang) Lung Cheung Hsien, elev. 500 ft. 24 September 1920, H.H.Hu 439 (K); south-west Chekiang, 18 August 1924, R.C.Ching 2432 for A.Rehder (K); Fatze-chiao, 7 July 1915, F.N.Meyer 1516 (K); (as Chekiang) Shihpu, 11 August 1927, C.Y.Chiao 14114 (K); Ningbo, August 1887 E[rnst] Faber 329 (K); (as Chekiang), without locality, H.H.Hu 1920 (K). 
VIETNAM: Tonkin, Kau-Nga-shan and vicinity, Tien-yen, 23 September to 7 October 1940, W.T.Tsang 30541 (K).

\section{Vouchers used for Figure 2. Distinctive morphological characters in Tribe Wis- terieae}

Endosamara racemosa Thailand, Prae, elev. 600-1000 ft. April 1910, Luang Vanpruk 188 (K); Padbruggea dasyphylla Myanmar, Perak, Scortechini 429 (K); Malaysia [Borneo], Sabah, Tenom, Palang-Palang hills, 4 January 1991, Lamb 395/91 (K); Austrocallerya australis Papua New Guinea, eastern highlands, distr. track for Arau-Obura elev. 1500m. 18 October 1959, L.J.Brass 32129, (K); Austrocallerya pilipes Australia, Queensland, SFR 143, Parish of Riflemead, Lerra, LA, 3 July 1986, B.Gray 04319 (K); Padbruggea filipes Myanmar, Sone Lone Taung, 25 February 1927, Maung Po Khant 15326 (K); Afgekia sericea Thailand, Bankhen, Bangkok 9 September 1968, C.Chermserivithana 996 (K); Thailand, May 1935, Mrs Collins 104/9 for A.F.G.Kerr (K); Callerya nitida China, Hong Kong, 23 September 1885, Theophilus Sampson s.n. (K); Callerya cinerea Tibet, 1917-1919, George Forrest 19279 (K); Whitfordiodendron nieuwenhuisii Indonesia [Borneo], Kalimantan, $5 \mathrm{~km}$ west of Batu, Badingding, 19 December 1982, J.P.Mogea 4182 (K); Whitfordiodendron erianthum Thailand, Songkhla Prov., image from photograph Y.Sirichamorn s.n.; Wisteriopsis eurybotrya Vietnam, Tourane, May-July 1927, J.\& M.S.Clemens 3637 (K); Wisteriopsis championii China, Hong Kong, Fo-tan south valley, 20 June 1970, Shiu Ying Hu 10476 (K).

\section{Supplementary material I}

\section{Figures S1-S6}

Authors: James A. Compton, Brian D. Schrire, Kálmán Könyves, Félix Forest, Panagiota Malakasi, Sawai Mattapha, Yotsawate Sirichamorn

Data type: molecular data

Explanation note: Fig. S1. Combined ML Bootstrap tree of Tribe Wisterieae and outgroups. Fig. S2. Combined Bayesian Inference (BPP) tree of tribe Wisterieae and outgroups. Fig. S3. Plastid ML Bootstrap tree of Tribe Wisterieae and outgroups. Fig. S4. Plastid Bayesian Inference (BPP) tree of Tribe Wisterieae and outgroups. Fig. S5. ITS ML Bootstrap tree of Tribe Wisterieae and outgroups. Fig. S6. ITS Bayesian Inference (BPP) tree of Tribe Wisterieae and outgroups.

Copyright notice: This dataset is made available under the Open Database License (http://opendatacommons.org/licenses/odbl/1.0/). The Open Database License $(\mathrm{ODbL})$ is a license agreement intended to allow users to freely share, modify, and use this Dataset while maintaining this same freedom for others, provided that the original source and author(s) are credited.

Link: https://doi.org/10.3897/phytokeys.125.34877.suppl1 